\title{
A Hybrid Al/ML and Computational Mechanics Based Approach for Time-Series State and Fatigue Life Estimation of Nuclear Reactor Components
}

LWRS milestone Report Number: M2LW-200R0402113

Nuclear Science and Engineering Division Argonne National Laboratory 


\begin{abstract}
About Argonne National Laboratory
Argonne is a U.S. Department of Energy laboratory managed by UChicago Argonne, LLC under contract DE-AC02-06CH11357. The Laboratory's main facility is outside Chicago, at 9700 South Cass Avenue, Argonne, Illinois 60439. For information about Argonne and its pioneering science and technology programs, see www.anl.gov.
\end{abstract}

\title{
DOCUMENT AVAILABILITY
}

Online Access: U.S. Department of Energy (DOE) reports produced after 1991 and a growing number of pre-1991 documents are available free at OSTI.GOV (http://www.osti.gov/), a service of the US Dept. of Energy's Office of Scientific and Technical Information.

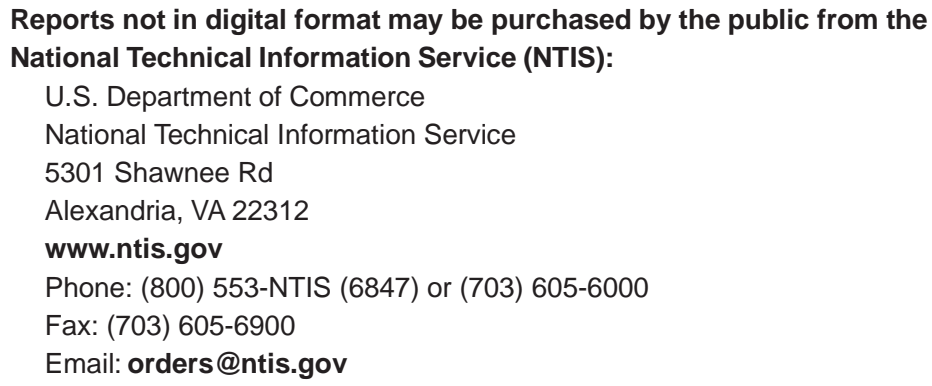

Reports not in digital format are available to DOE and DOE contractors from the Office of Scientific and Technical Information (OSTI):

U.S. Department of Energy

Office of Scientific and Technical Information

P.O. Box 62

Oak Ridge, TN 37831-0062

www.osti.gov

Phone: (865) 576-8401

Fax: (865) 576-5728

Email: reports@osti.gov

\section{Disclaimer}

This report was prepared as an account of work sponsored by an agency of the United States Government. Neither the United States Government nor any agency thereof, nor UChicago Argonne, LLC, nor any of their employees or officers, makes any warranty, express or implied, or assumes any legal liability or responsibility for the accuracy, completeness, or usefulness of any information, apparatus, product, or process disclosed, or represents that its use would not infringe privately owned rights. Reference herein to any specific commercial product, process, or service by trade name, trademark, manufacturer, or otherwise, does not necessarily constitute or imply its endorsement, recommendation, or favoring by the United States Government or any agency thereof. The views and opinions of document authors expressed herein do not necessarily state or reflect those of the United States Government or any agency thereof, Argonne National Laboratory, or UChicago Argonne, LLC. 


\section{A Hybrid Al/ML and Computational Mechanics Based Approach for Time-Series State and Fatigue Life Estimation of Nuclear Reactor Components}

LWRS milestone Report Number: M2LW-200R0402113

Subhasish Mohanty and Joseph Listwan

Nuclear Science and Engineering Division, Argonne National Laboratory

September 2020 
This page intentionally left blank 


\section{ABSTRACT}

Environmental fatigue modeling is a complex problem due to multiple failure modes and their intermixing. The failure modes are function of various underlying causes in addition to the corrosive effect of reactor coolant environment. Some of the major causes are time-dependence of material associated with cyclic loading, load sequence effect associated with random/variable amplitude loading, effect of strain amplitude and rates, effect of varying temperature (along both temporal and spatial directions) and the effect of mean strain and stress. The nonlinear intermixing of failure modes associated with above mentioned causing parameters makes the environmental fatigue modeling is a challenging task. Because of this challenge, fatigue is traditionally being modeled based on experimental data. However, test based empirical approach often requires hundreds of fatigue tests to model the above-mentioned intermixing failure causes even for a single material system. The problem is further exaggerated for reactor component made from multi-material systems such as made from both carbon and stainless-steel base metals and their similar and dissimilar metal welds. With the difficulty of conducting hundreds of fatigue tests to capture the above-mentioned intermixing failure causes, fatigue modeling approaches often depends on empirical models based on limited available test data such as available through ASME code and NUREG 6909. However, these limited test-data-based models may not be enough to accurately predict the life of reactor components. Accurate prediction of life of reactor component would become a necessity, particularly when the license of the reactors to be extended for long-term-operation (LTO) that is for well beyond its original design life of 40 years.

The requirement of extending the license of reactor under LTO requires hundreds of fatigue tests to be conducted to understand the mechanism associated with the above-mentioned interdependent failure causes. However, conducting large number of fatigue tests is not a feasibility due to the cost involved. To address this issues Argonne National Laboratory (ANL) with the sponsorship of DOE Light Water Reactor Sustainability (LWRS) program trying to develop a hybrid predictive modeling approach. This is based on limited experiment-data, Artificialintelligence (AI) - Machine-Learning (ML) - Deep-Learning (DL) based techniques and Multiphysics-computational-mechanics based modeling tools. The hybrid approach not-only can improve the accuracy of the existing stress analysis and fatigue modeling approach but also can reduce the over-dependency on test-based approach. Towards this goal following are some of the major contributions based on ANL's FY-20 environmental fatigue modeling activities:

1) A cyclic plasticity material model database for $82 / 182$ dissimilar metal weld, which can be readily shared with US nuclear industry and regulatory agency on request.

2) A well validated analytical modeling methodology to perform cycle-by-cycle stress prediction under both constant amplitude fatigue loading and variable amplitude fatigue loading (with load-sequence effect).

3) An AI/ML/DL based methodology to predict unmeasurable cyclic strain based on other available sensor signals. This type of approach can be used for estimating strain in real reactor components from other sensor readings.

4) An AI/ML based approach to improve the US capability on environmental fatigue testing. This is by improving ANL's existing environmental fatigue testing capacity to conduct 
ASME required strain-controlled tests (by controlling strain amplitudes and its rate), while not measuring the strain (due to the difficulty of placing an extensometer in a narrow autoclave in a PWR-water-test system).

5) A simulation and experiment based probabilistic modeling methodology for time-series fatigue state and life estimation of reactor metal such as dissimilar metal weld. 


\section{TABLE OF CONTENTS}

A Hybrid Al/ML and Computational Mechanics Based Approach for Time-Series State and Fatigue Life Estimation of Nuclear Reactor Components

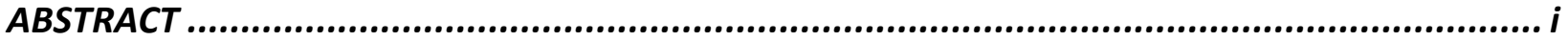

Table of Contents ..................................................................................... iii

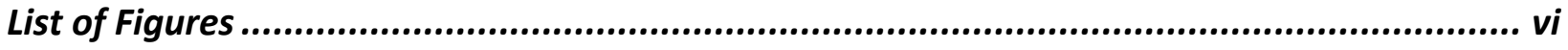

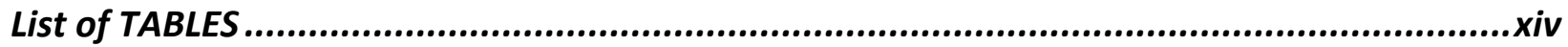

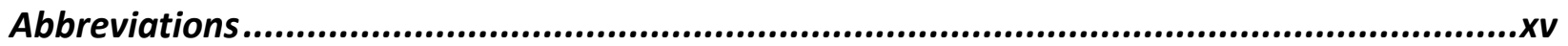

Acknowledgments ......................................................................................

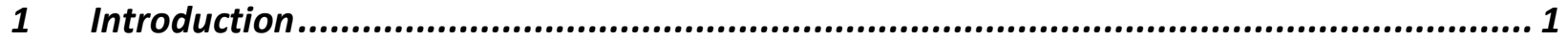

2 Brief Theoretical Background of the AI/ML/DL Techniques Used in the Discussed Work .. 2

2.1 Justification Behind Using AI/ML/DL Techniques for Fatigue Modeling ..................................2

2.2 Brief Introduction to Multi-Layer Perceptron in Context of Time-Dependent Fatigue Data

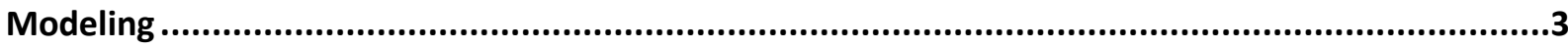

2.3 Use of Scikit-learn-Regression Library for Time-Series Fatigue Data Prediction ..........................6

2.4 Use of Keras Deep Learning Library for Time-Series Fatigue Data Prediction...............................6

2.5 Use of TensorFlow Library for Cyclic Stress-Strain Curve Prediction ...........................................7

2.6 Use of Scikit-learn Library Based k-Mean Clustering Technique for Optimizing Fatigue-Test Control Inputs and Improving Time-Series Fatigue Data Prediction Techniques..............................8

2.7 Markov-Chain-Monte-Carlo Based Probabilistic Fatigue Life Prediction...................................9

3 Loading and Environment of Different Experimental Fatigue Test Cases...................... 10

3.1 Justification for Selecting Strain Amplitudes for Different Fatigue Testing ...............................10

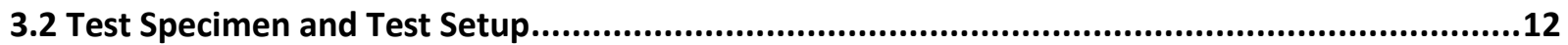

3.3 Loading Inputs and Environment for Different Test Cases .....................................................14

$4 \quad$ Al/ML Guided Seemingly Strain-Controlled Fatigue Test While Actually Not Controlling the Strain ................................................................................................. 20

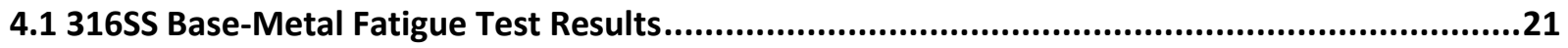

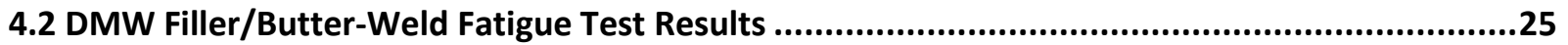

5 Use of Al-Based Machine Learning and Deep Learning Techniques for Time-Series Strain Prediction from Other Sensor Measurements......................................................... 29

5.1 Validation case-1: Cyclic Strain Prediction for Fatigue Test Specimen Subjected to ET-F54 Unsymmetrical Design-Basis Cyclic Loading

5.1.1 Study the effect of independent variables on accuracy of strain estimation under unsymmetrical design-basis cyclic loading ..... 
A Hybrid Al/ML and Computational Mechanics Based Approach for Time-Series State and Fatigue Life Estimation of Nuclear

5.1.2 Accuracy associated with different types of Al technique under unsymmetrical design-basis cyclic loading 33

5.1.3 Clustering and piecewise strain time-series estimation to deal with non-stationarity associated with cyclic hardening under unsymmetrical design-basis cyclic loading .....

5.2 Validation case-2: Cyclic Strain Estimation for Fatigue Test Specimen Subjected to ET-F61 Symmetrical Cyclic Loading

5.2.1 Study the effect of independent variables on accuracy of strain estimation under symmetrical cyclic loading 45

5.2.2 Accuracy associated with different type of Al techniques under symmetrical cyclic loading ..... .48

5.2.3 Clustering and piecewise strain time-series estimation to deal with non-stationarity associated with

cyclic hardening under symmetrical cyclic loading..... .55

5.3 Prediction Case: Cyclic Strain Prediction for Fatigue Test Specimen Subjected to EN-F62

Symmetrical Cyclic Loading and under PWR-Coolant-Water Environment. .60

6 A Hybrid Al-TensorFlow and Computational-Mechanics Based Approach for Cyclic Material Parameter Prediction ........................................................................... 63

6.1 Material Model Theoretical Background .63

6.2 Results: Use of TensorFlow to Estimate the Cyclic-Stress-Strain Curves of DMW. .64

6.3 Results: Use of Computational-Mechanics Based Approach to Estimate Elastic-Plastic Material

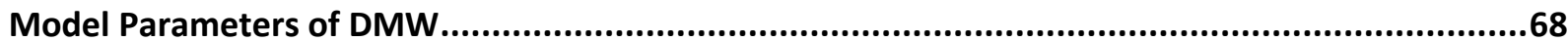

6.3.1 Example estimation of material model parameters for $1^{\text {st }}$-block ...................................................69

6.3.2 Estimated material parameters for entire fatigue life and considering $0.05 \%$ offset yield limit.........71

6.3.3 Automated material parameter data saving into SQL database ......................................................74

7 Analytical Model Based Cyclic Stress Analysis of Different DMW Test Cases under Constant and Variable Amplitude Cyclic Loading ..................................................... 75

7.1 Cyclic Stress Analysis Results for ET-F57 DMW Specimen Subjected to Gauge-area-strain-control

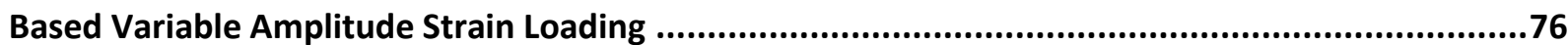

7.1.1 Cyclic stress analysis results for ET-F57 specimen using fixed tensile properties ...........................78

7.1.2 Cyclic stress analysis results for ET-F57 specimen using variable scaled-cycle-dependent material model properties

7.2 Cyclic Stress Analysis Results for ET-F59 DMW Specimen Subjected to Gauge-area-straincontrol Based Constant Amplitude Strain Loading.

7.2.1 Cyclic stress analysis results for ET-F59 specimen using fixed tensile properties .............................82

7.2.2 Cyclic stress analysis results for ET-F59 specimen using variable scaled-cycle-dependent material model properties.

7.3 Cyclic Stress Analysis Results for ET-F60 DMW Specimen Subjected to Pull-rod-control Based Constant Amplitude Displacement Loading

7.3.1 Cyclic stress analysis results for ET-F60 specimen using fixed tensile properties .............................87

7.3.2 Cyclic stress analysis results for ET-F60 specimen using variable scaled-cycle-dependent material model properties.

7.4 Cyclic Stress Analysis Results for ET-F61 DMW Specimen Subjected to AI-Guided and Pullrod-control Based Variable Amplitude Displacement Loading ................................................90

7.4.1 Cyclic stress analysis results for ET-F61 specimen using fixed tensile properties ............................91

7.4.2 Cyclic stress analysis results for ET-F61 specimen using variable cyclic-plasticity material model properties. 
7.5 Cyclic Stress Analysis Results for ET-F57 DMW Specimen Based on Accumulated Strainenergy Dependent Material Models

7.5.1 Accumulated strain-energy dependent material properties

7.5.2 Cyclic stress analysis results for ET-F57 specimen using accumulated strain-energy dependent material model properties

8 Demonstration of the Use of Cyclic-Plasticity Material Parameters in 3D FE Model of a PWR Surge-line Nozzle.................................................................................. 98

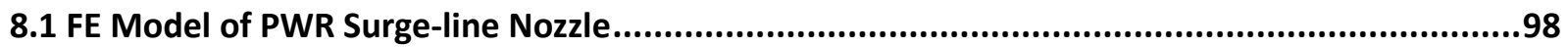

8.2 FE stress analysis results with respect to ET-F57 block-1 and block-68 (half-life) properties ....101

9 Markov-chain-Monte-Carlo Based Probabilistic-Time-Series State and Fatigue Life Estimation of DMW ................................................................................. 105

9.1 Results: MCMC Model Simulation Based on Data of ET-F57 Analytical Model (with Accumulated Strain Energy Dependent Cyclic-Plasticity Material Properties)...............................................107

9.2 Results: MCMC Model Simulation Based on ET-F57 In-air Fatigue Test Data ............................108

9.3 Results: MCMC Model Simulation Based on EN-F62 PWR-water Fatigue Test Data ..................109

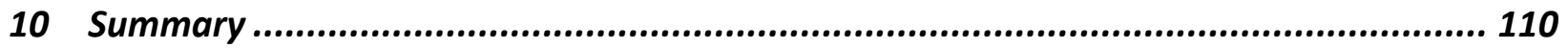

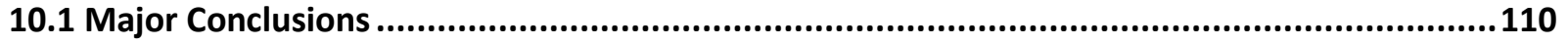

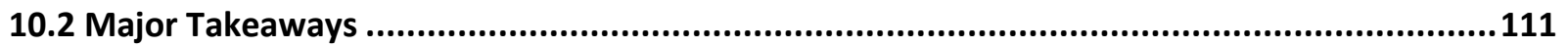

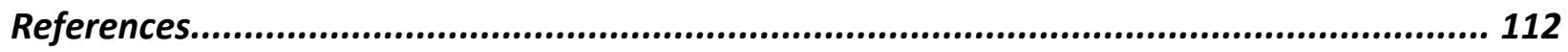




\section{LIST OF FIGURES}

Figure 2. 1 Schematic of the difference between of AI, ML and DL techniques...................... 3 Figure 2. 2 Example strain versus stress hysteresis data for 316SS-508LAS dissimilar metal weld subjected to variable amplitude loading.

Figure 2. 3 Schematic of an MLP network with time-varying fatigue damage causing inputs (e.g. strain, temperature, etc.) and resulting observed outputs (e.g. stress). ............................... 5

Figure 2. 4 Schematic showing the difference between global optima and local optima trap. ...... 5

Figure 2. 5 Schematic showing the relation between Keras and TensorFlow library. .................. 7

Figure 2. 6 Example two-dimensional data sets with four cluster........................................ 9

Figure 3. 1 FE model (case-1) simulated total, thermal, and mechanical strain (along max. principal direction) at the maximum stress element of the DMW-filler: (a) full design-basis cycle, (b) during heat-up, and (c) during cool-down [17]............................................. 11

Figure 3. 2 Maximum principal thermal strain contour plot at 1.3717 days (32.921 hours) from the start of the heat-up operation showing the DMW region experiences least thermal strain

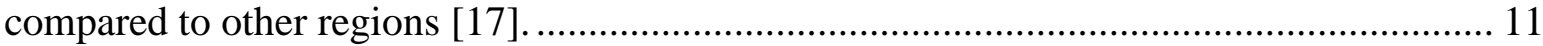

Figure 3. 3 Cross-section of the weld plates (only near the weld region) and location of the along-the-length weld and HAZ specimens............................................................... 12

Figure 3. 4 Geometry of hourglass-type specimens............................................................. 12

Figure 3.5 a) Test section with induction heating coil, b) LEPEL induction heating system, and c) close view of induction heating coil and specimen and high-temperature extensometer

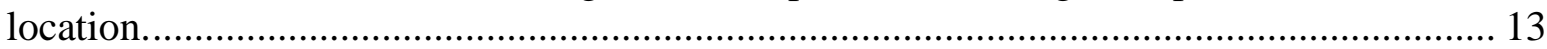

Figure 3. 6 Environmental test loop showing different subsystems.................................... 13

Figure 3. 7 Pull-rod-displacement that was applied to ET-F13 (316SS base) specimen until its

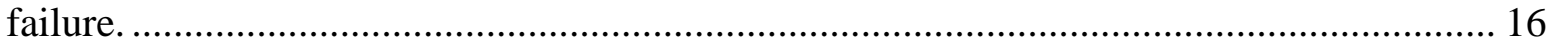

Figure 3. 8 Improved pull-rod-displacement (and corresponding intended strain amplitude) that was applied to ET-F49 (316SS base) specimen until its failure.................................... 17

Figure 3. 9 Pull-rod displacement (and corresponding intended strain amplitude) that was applied to ET-F54 (82/182 DMW filler weld) specimen over the entire fatigue life..................... 17

Figure 3. 10 A single-block of variable amplitude gage-area strain that was applied to ET-F57 (82/182 DMW filler weld) specimen until its failure.

Figure 3. 11 Example first cycle input (out of different inputs applied to different cycles selected based on AI/ML based clustering technique) of pull-rod-displacement that was applied to ET-F58 (316SS base) specimen. 18

Figure 3. 12 A single-block of constant amplitude gage-area strain that was applied to ET-F59 (82/182 DMW butter weld) specimen until its failure.

Figure 3. 13 Pull-rod-displacement (and corresponding intended strain amplitude) that was applied to ET-F60 (82/182 DMW filler weld) specimen until its failure......................... 19

Figure 3. 14 Example first cycle input (out of different inputs applied to different cycles selected based on AI/ML based clustering technique) of pull-rod-displacement that was applied to ET-F61 \& EN-F62 (82/182 DMW filler weld) specimens........................................... 20

Figure 4. 1 A 316SS fatigue-test (ET-F49) based clustered data showing the example 2dimensional dependency between frame maximum actuator displacement and corresponding specimen gage area maximum strain. Raw data in an individual cluster 
(plotted with single color) are reparented by dots whereas, the solid circles represent the corresponding cluster center. 22

Figure 4. 2 Cycle versus corresponding cluster numbers showing how the entire ET-F49 fatiguetest data clustered into only 5 different groups/clusters based on which the ET-F58 test parameters were chosen.

Figure 4. 3 Observed cycle versus strain amplitude in different fatigue tests conducted using

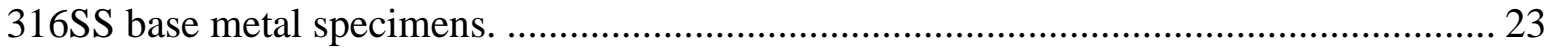

Figure 4. 4 Observed cycle versus strain rate in different fatigue tests conducted using 316SS base metal specimens.................................................................................... 24

Figure 4. 5 Observed cycle versus max./min stress in different fatigue tests conducted using

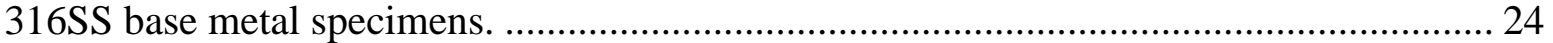

Figure 4. 6 A DMW fatigue-test (ET-F60) based clustered data showing the example 2dimensional dependency between frame maximum actuator displacement and corresponding specimen gage area maximum strain. Raw data in an individual cluster (plotted with single color) are reparented by dots whereas, the solid circles represent the corresponding cluster center. 26

Figure 4. 7 Cycle versus corresponding cluster numbers showing how the entire ET-F60 fatiguetest data clustered into only 9 different groups/clusters based on which the ET-F61 and ENF62 test parameters were chosen. .................................................................. 26

Figure 4. 8 Observed cycle versus strain amplitude in different fatigue tests conducted using

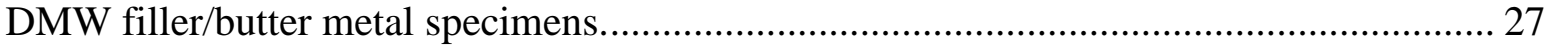

Figure 4. 9 Observed cycle versus strain rate in different fatigue tests conducted using DMW

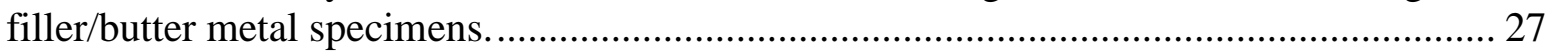

Figure 4. 10 Observed cycle versus max./min stress in different fatigue tests conducted using DMW filler/butter metal specimens.......................................................................... 28

Figure 5. 1 Cyclic strain estimation using the time as the only independent variable................ 31

Figure 5. 2 Cyclic strain estimation using displacement of the test-frame actuator as the only

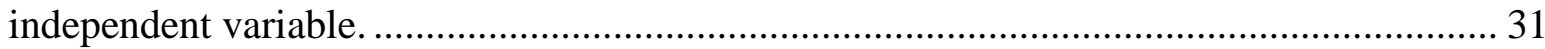

Figure 5. 3 Cyclic strain estimation using the load-cell measurement of the test-frame as the only

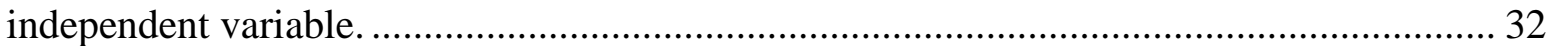

Figure 5. 4 Cyclic strain estimation using the pull-rod displacement as the only independent

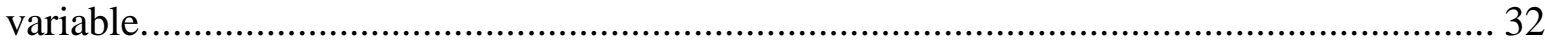

Figure 5. 5 Scikit-learn library-based MSE (with respect to different randomized sampling iterations), while predicting strain for first 50 cycles of ET-F54 test case........................ 34

Figure 5. 6 Scikit-learn library-based predicted strain (for first 50 cycles of ET-F54 test case) and its comparison to corresponding experimental strain. ............................................ 34

Figure 5. 7 Magnified version of Figure 5.6.................................................................. 35

Figure 5. 8 Scikit-learn library-based predicted strain hysteresis curves (for first 50 cycles of ETF54 test case) and its comparison to corresponding experimental strain hysteresis curves. 35 Figure 5. 9 Keras library and Sigmoid activation-function based MSE (with respect to different randomized sampling iterations), while predicting strain for first 50 cycles of ET-F54 test

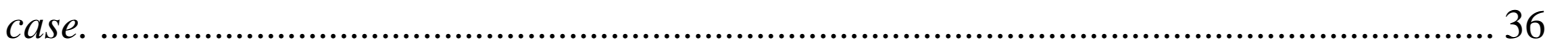

Figure 5. 10 Keras library and Sigmoid activation-function based predicted strain (for first 50 cycles of ET-F54 test case) and its comparison to corresponding experimental strain...... 36

Figure 5. 11 Magnified version of Figure 5.10 ................................................................... 37 
Figure 5. 12 Keras library and Sigmoid activation-function based hysteresis curves (for first 50 cycles of ET-F54 test case) and its comparison to corresponding experimental strain hysteresis curves. 37

Figure 5. 13 Keras library and ReLU activation-function based MSE (with respect to different randomized sampling iterations), while predicting strain for first 50 cycles of ET-F54 test

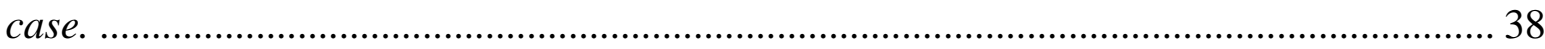

Figure 5. 14 Keras library and ReLU activation-function based predicted strain (for first 50 cycles of ET-F54 test case) and its comparison to corresponding experimental strain....... 38

Figure 5. 15 Magnified version of Figure 5.14.................................................................. 39

Figure 5. 16 Keras library and ReLU activation-function based hysteresis curves (for first 50 cycles of ET-F54 test case) and its comparison to corresponding experimental strain hysteresis curves. 39

Figure 5. 17 Substantial variation of mean of ET-F54 cyclic strains shows that the time-series strain for the entire fatigue life follows a nonstationary process. .................................. 40

Figure 5. 18 Substantial variation of standard deviation of ET-F54 cyclic strains shows that the time-series strain for the entire fatigue life follows a nonstationary process. ................... 41

Figure 5. 19 ET-F54 clustered data showing the 2-dimensional dependency between frame maximum actuator displacement and corresponding specimen gage area maximum strain. Raw data in an individual cluster (plotted with a single color) are reparented by dots whereas, the solid circles represent the corresponding cluster center.

Figure 5. 20 Cycle versus corresponding cluster numbers showing how the entire ET-F54 fatigue-test data clustered into only 10 different groups/clusters. Piecewise strain prediction models were developed for individual clusters to predict the strain for the entire fatigue life of ET-F54 test case. 42

Figure 5. 21 Keras library and ReLU activation-function based predicted time-series strain (for the entire fatigue cycles of ET-F54 test case) and its comparison to corresponding experimental strain. 42

Figure 5. 22 Magnified version of Figure 5.21 showing first few cycles (of ET-F54 test case) prediction versus experimental results.

Figure 5. 23 Magnified version of Figure 5.21 showing few half-life cycles (of ET-F54 test case) prediction versus experimental results..................................................................... 43

Figure 5. 24 Magnified version of Figure 5.21 showing few end-life cycles (of ET-F54 test case) prediction versus experimental results........................................................................ 44

Figure 5. 25 Predicted strain hysteresis curves (for entire fatigue life of ET-F54 test case) and its comparison to corresponding experimental strain hysteresis curves................................ 44

Figure 5. 26 Cyclic strain estimation using the time as the only independent variable. ............. 46

Figure 5. 27 Cyclic strain estimation using displacement of the test-frame actuator as the only independent variable ...................................................................................................... 46

Figure 5. 28 Cyclic strain estimation using the load-cell measurement of the test-frame as the only independent variable ................................................................................................. 47

Figure 5. 29 Cyclic strain estimation using the pull-rod displacement as the only independent

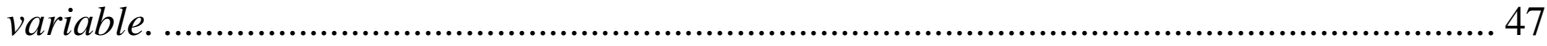

Figure 5. 30 Scikit-learn library-based MSE (with respect to different randomized sampling iterations), while predicting strain for first 50 cycles of ET-F61 test case ......................... 49

Figure 5. 31 Scikit-learn library-based predicted strain (for first 50 cycles of ET-F61 test case) and its comparison to corresponding experimental strain. ............................................ 49 
Figure 5. 32 Magnified version of Figure 5.31 .................................................................. 50

Figure 5. 33 Scikit-learn library-based predicted strain hysteresis curves (for first 50 cycles of ET-F61 test case) and its comparison to corresponding experimental strain hysteresis curves.

Figure 5. 34 Keras library and Sigmoid activation-function based MSE (with respect to different randomized sampling iterations), while predicting strain for first 50 cycles of ET-F61 test

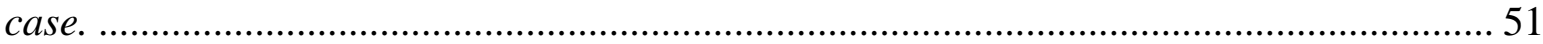

Figure 5. 35 Keras library and Sigmoid activation-function based predicted strain (for first 50 cycles of ET-F61 test case) and its comparison to corresponding experimental strain....... 51

Figure 5. 36 Magnified version of Figure 5.35 ...................................................................... 52

Figure 5. 37 Keras library and Sigmoid activation-function based hysteresis curves (for first 50 cycles of ET-F61 test case) and its comparison to corresponding experimental strain

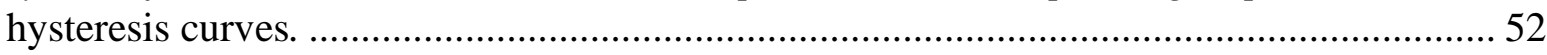

Figure 5. 38 Keras library and ReLU activation-function based MSE (with respect to different randomized sampling iterations), while predicting strain for first 50 cycles of ET-F61 test case ................................................................................................................ 53

Figure 5. 39 Keras library and ReLU activation-function based predicted strain (for first 50 cycles of ET-F61 test case) and its comparison to corresponding experimental strain....... 53

Figure 5. 40 Magnified version of Figure 5.39 .................................................................... 54

Figure 5. 41 Keras library and ReLU activation-function based hysteresis curves (for first 50 cycles of ET-F61 test case) and its comparison to corresponding experimental strain

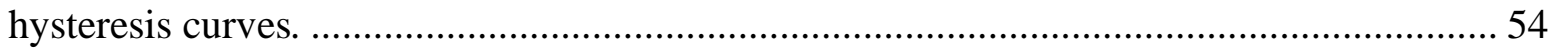

Figure 5. 42 Substantial variation of mean of ET-F61 cyclic strains shows that the time-series strain for the entire fatigue life follows a non-stationary process......................................... 55

Figure 5. 43 Variation of standard deviation of ET-F61 cyclic strains shows that the time-series strain for the entire fatigue life follows a non-stationary process........................................ 56

Figure 5. 44 ET-F61 clustered data showing the 2-dimensional dependency between frame maximum actuator displacement and corresponding specimen gage area maximum strain. Raw data in an individual cluster (plotted with a single color) are reparented by dots whereas, the solid circles represent the corresponding cluster center. 56

Figure 5. 45 Cycle versus corresponding cluster numbers showing how the entire ET-F61 fatigue-test data clustered into only 10 different groups/clusters. Piecewise strain prediction models were developed for individual clusters to predict the strain for the entire fatigue life of ET-F61 test case. 57

Figure 5. 46 Keras library and ReLU activation-function based predicted time-series strain (for the entire fatigue cycles of ET-F61 test case) and its comparison to corresponding experimental strain.

Figure 5. 47 Magnified version of Figure 5.46 showing first few cycles (of ET-F61 test case) prediction versus experimental results........................................................................ 58

Figure 5. 48 Magnified version of Figure 5.46 showing few half-life cycles (of ET-F61 test case) prediction versus experimental results.......................................................................... 58

Figure 5. 49 Magnified version of Figure 5.46 showing few end-life cycles (of ET-F61 test case) prediction versus experimental results............................................................................ 59

Figure 5. 50 Predicted strain hysteresis curves (for entire fatigue life of ET-F61 test case) and its comparison to corresponding experimental strain hysteresis curves.................................. 59 
Figure 5. 51 Keras library and ReLU activation-function based predicted time-series strain for the entire fatigue cycles of EN-F62 test case.

Figure 5. 52 Magnified version of Figure 5.51 showing strain prediction results for first few cycles of EN-F62 test case. 61

Figure 5. 53 Magnified version of 5.51 showing strain prediction results for few half-life cycles of EN-F62 test case.

Figure 5. 54 Magnified version of 5.51 showing strain prediction results for few end-life cycles of EN-F62 test case. 62

Figure 5. 55 Predicted strain hysteresis curves for entire fatigue life of EN-F62 test case. 62

Figure 6. 1 Repeating one-block (comprising 12 increasing/decreasing cycles) of input strain that was applied to ET-F57 DMW filler weld specimen.

Figure 6. 2 Observed (output) first block (comprising 12 increasing/decreasing cycles) of stress

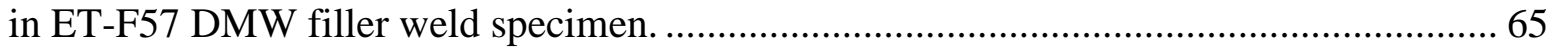

Figure 6. 3 Underlying input-output (or strain versus stress hysteresis) data and the corresponding TensorFlow predicted equivalent cyclic-stress-strain curve for $1^{\text {st }}$-block of

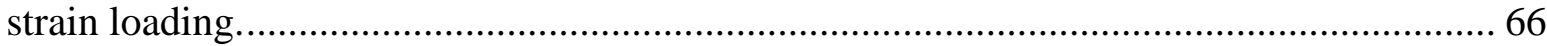

Figure 6. 4 Training iteration no. versus estimated bias for $1^{\text {st }}$-block of strain loading.............. 66 Figure 6. 5 Training iteration no. versus estimated loss function for $1^{\text {st }}$-block of strain loading. 67 Figure 6. 6 Estimated up/down-cycle equivalent-monotonic stress-strain curve for $1^{\text {st }}$-block of strain loading.....

Figure 6. 7 Example results showing how good/bad the linear and nonlinear kinematic hardening $(\mathrm{KH})$ models capture the plastic strain versus back stress curve of $1^{\text {st }}$-block strain loading.69

Figure 6. 8 Iteration no. (in a Newton optimization process) versus estimated $\mathrm{C} 1$ parameter showing convergence of $\mathrm{C} 1$ parameter for $1^{\text {st }}$-block strain loading.

Figure 6. 9 Iteration no. (in a Newton optimization process) versus estimated $\gamma 1$ parameter showing convergence of $\gamma 1$ parameter for $1^{\text {st }}$-block strain loading. ................................ 70

Figure 6. 10 Iteration no. (in a Newton optimization process) versus estimated L2 norm of $\Delta \mathrm{C} 1$ and $\Delta \gamma 1$ showing convergence of estimated parameters for $1^{\text {st }}$-block strain loading.......... 70

Figure 6. 11 Evolution of equivalent monotonic stress-strain curves (in red) and the corresponding $0.05 \%$ offset yield line (in green) for the entire ET-F57 fatigue life showing the need of cyclic material models.

Figure 6. 12 Block no. versus estimated elastic modulus with assumption of $0.05 \%$ offset yield limit.

Figure 6. 13 Block no. versus estimated offset yield stress with assumption of $0.05 \%$ offset yield limit. 72

Figure 6. 14 Block no. versus estimated linear kinematic hardening parameter $\mathrm{C} 1$ with assumption of $0.05 \%$ offset yield limit. ............................................................. 72

Figure 6. 15 Block no. versus estimated nonlinear kinematic hardening parameter $\mathrm{C} 1$ with assumption of $0.05 \%$ offset yield limit. ................................................................... 73

Figure 6. 16 Block no. versus estimated nonlinear kinematic hardening parameter $\gamma 1$ with

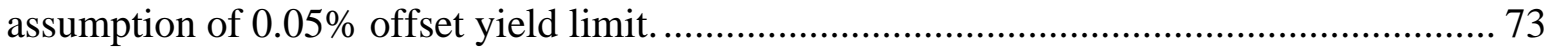

Figure 6. 27 Snapshot of the automatically updated SQL based material database................... 74

Figure 7. 1 Example first 5 blocks ET-F57 input strain loading. ......................................... 76

Figure 7. 2 Example first 5 blocks ET-F57 observed stress................................................ 77 
Figure 7. 3 Block no. versus maximum cyclic strain amplitude (both applied to analytical model and experimentally observed during the fatigue test of ET-F57 specimen) to track any anomaly in strain loading. 77

Figure 7. 4 Fixed tensile properties based predicted versus experimentally observed cyclic stress for the entire fatigue life of ET-F57 test case. 78

Figure 7. 5 Magnified version of Figure 7.4, approximately during the half-life of ET-F57 specimen. .78

Figure 7. 6 Fixed tensile properties based predicted versus experimentally observed max./min stress-amplitudes for the entire fatigue life of ET-F57 test case (The filtered data is to remove any anomaly associated with model prediction).

Figure 7. 7 Cycle-dependent properties based predicted versus experimentally observed cyclic stress for the entire fatigue life of ET-F57 test case. 79

Figure 7. 8 Magnified version of Figure 7.7, approximately during the half-life of ET-F57

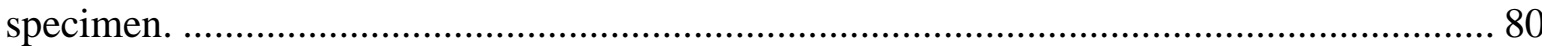

Figure 7. 9 Cycle-dependent properties based predicted versus experimentally observed max./min stress-amplitudes for the entire fatigue life of ET-F57 test case. ........................... 80

Figure 7. 10 Example first 5 cycles ET-F59 input strain loading............................................... 81

Figure 7. 11 Example first 5 cycles ET-F59 observed stress................................................... 81

Figure 7. 12 Cycle no. versus maximum cyclic strain amplitude (both applied to analytical model and experimentally observed during the fatigue test of ET-F59 specimen) to track any anomaly in strain loading. 82

Figure 7. 13 Fixed tensile properties based predicted versus experimentally observed cyclic stress for the entire fatigue life of ET-F59 test case. 82

Figure 7. 14 Magnified version of Figure 7.13, approximately during the half-life of ET-F59

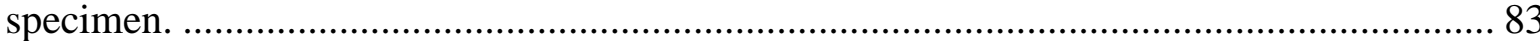

Figure 7. 15 Fixed tensile properties based predicted versus experimentally observed max./min stress-amplitudes for the entire fatigue life of ET-F59 test case. ......................................... 83

Figure 7. 16 Cycle-dependent properties based predicted versus experimentally observed cyclic stress for the entire fatigue life of ET-F59 test case. 84

Figure 7. 17 Magnified version of Figure 7.16, approximately during the half-life of ET-F59 specimen. 84

Figure 7. 18 Cycle-dependent properties based predicted versus experimentally observed max./min stress-amplitudes for the entire fatigue life of ET-F59 test case. .......................... 85

Figure 7. 19 Example first 5 cycles ET-F60 input strain loading.............................................. 85

Figure 7. 20 Example first 5 cycles ET-F60 observed stress................................................... 86 Figure 7. 21 Cycle no. versus maximum strain amplitude (both applied to analytical model and experimentally observed during the fatigue test of ET-F60 specimen) to track any anomaly

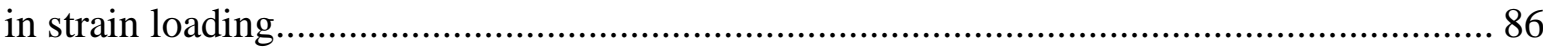

Figure 7. 22 Fixed tensile properties based predicted versus experimentally observed cyclic stress for the entire fatigue life of ET-F60 test case.

87

Figure 7. 23 Magnified version of Figure 7.22, approximately during the half-life of ET-F60

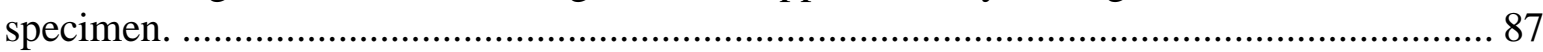

Figure 7. 24 Fixed tensile properties based predicted versus experimentally observed max./min stress-amplitudes for the entire fatigue life of ET-F60 test case. ........................................ 88

Figure 7. 25 Cycle-dependent properties based predicted versus experimentally observed cyclic stress for the entire fatigue life of ET-F60 test case. 
Figure 7. 26 Magnified version of Figure 7.25, approximately during the half-life of ET-F60

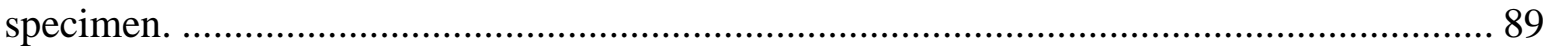

Figure 7. 27 Cycle-dependent properties based predicted versus experimentally observed max./min stress-amplitudes for the entire fatigue life of ET-F60 test case. .................... 89

Figure 7.28 Example first 5 cycles ET-F61 input strain loading......................................... 90

Figure 7. 29 Example first 5 cycles ET-F61 observed stress................................................ 90

Figure 7. 30 Cycle no. versus maximum cyclic strain amplitude (both applied to analytical model and experimentally observed during the fatigue test of ET-F61 specimen) to track any

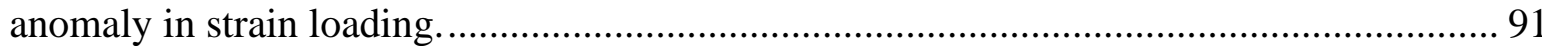

Figure 7. 31 Fixed tensile properties based predicted versus experimentally observed cyclic stress for the entire fatigue life of ET-F61 test case.

Figure 7. 32 Magnified version of Figure 7.31, approximately during the half-life of ET-F61

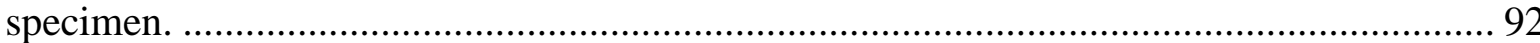

Figure 7. 33 Fixed tensile properties based predicted versus experimentally observed max./min stress-amplitudes for the entire fatigue life of ET-F61 test case. ..................................... 92

Figure 7. 34 Cycle-dependent properties based predicted versus experimentally observed cyclic stress for the entire fatigue life of ET-F61 test case.

Figure 7. 35 Magnified version of Figure 7.34, approximately during the half-life of ET-F61 specimen.

Figure 7. 36 Cycle-dependent properties based predicted versus experimentally observed max./min stress-amplitudes for the entire fatigue life of ET-F61 test case.

Figure 7. 37 Accumulated strain-energy versus elastic modulus, estimated based on the ET-F57 test data.

Figure 7. 38 Accumulated strain-energy versus $0.05 \%$ offset yield stress, estimated based on the ET-F57 test data.

Figure 7. 39 Accumulated strain-energy versus nonlinear kinematic hardening parameter C1,

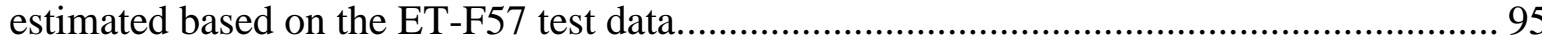

Figure 7. 40 Accumulated strain-energy versus nonlinear kinematic hardening parameter $\gamma 1$,

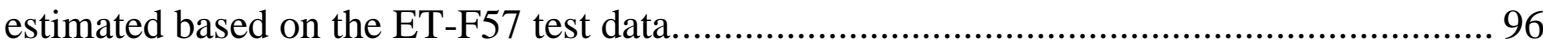

Figure 7. 41 Cycle-dependent properties based predicted versus experimentally observed cyclic stress for the entire fatigue life of ET-F57 test case. 96

Figure 7. 42 Magnified version of Figure 7.41, approximately during the half-life of ET-F57 specimen.

Figure 7. 43 Cycle-dependent properties based predicted versus experimentally observed max./min stress-amplitudes for the entire fatigue life of ET-F57 test case.

Figure 8. 1 Partial CAD model of PWR pressure control system showing the location of the surge line nozzle.

Figure 8. 2 CAD model of surge line nozzle and bottom head of pressurizer........................... 99

Figure 8. 3 Cut section of the nozzle assembly showing different material systems. .............. 100

Figure 8. 4 Cut section of the nozzle assembly finite element mesh................................... 100

Figure 8. 5 Demonstration showing how the estimated material properties can directly be used in a commercially available software such as ABAQUS. 101

Figure 8. 6 Cut section of nozzle assembly showing the location and direction of the applied variable-amplitude displacements to simulate variable-amplitude strain at the DMW filler weld. 102 
Figure 8. 7 Applied 1-block of displacement at the circular mid-plane of DMW filler region. . 102

Figure 8. $8 \mathrm{FE}$ simulated cycle versus strain at a typical element of DMW filler weld (of the PWR surge nozzle) subjected to ET-F57 $1^{\text {st }}$ block and $68^{\text {th }}$ block (half-life) material properties.

Figure 8. 9 FE simulated cycle versus stress at a typical element of DMW filler weld (of the PWR surge nozzle) subjected to ET-F57 $1^{\text {st }}$ block and $68^{\text {th }}$ block (half-life) material properties. 103

Figure 8. $10 \mathrm{FE}$ simulated stress versus strain at a typical element of DMW filler weld (of the PWR surge nozzle) subjected to ET-F57 $1^{\text {st }}$ block and $68^{\text {th }}$ block (half-life) material properties. 104

Figure 8. 11 Von. Mises stress (Pascal) contour of PWR surge-line nozzle at a typical instance of the applied variable amplitude displacement, while subjected to ET-F57 half-life (68 ${ }^{\text {th }}$ block) material properties. 104

Figure 9. $1 \mathrm{MCMC}$ estimated loading block versus the scatter-band of the analytically simulated maximum stress amplitude of ET-F57 test case. 107

Figure 9. 2 Loading block versus estimated lifetime CDF, estimated based on the scatter-band shown in Figure 9.1 and using different load-drop failure criterion. 107

Figure 9. 3 MCMC estimated loading block versus the scatter-band of experimentally observed maximum stress amplitude of ET-F57 test case. 108

Figure 9. 4 Loading block versus estimated lifetime CDF, estimated based on the scatter-band shown in Figure 9.3 and using different load-drop failure criterion. 108

Figure 9. 5 MCMC estimated loading cycle versus the scatter-band of experimentally observed maximum stress amplitude of EN-F62 test case 109

Figure 9. 6 Loading cycle versus estimated lifetime CDF, estimated based on the scatter-band shown in Figure 9.5 and using different load-drop failure criterion 109 


\section{LIST OF TABLES}

Table 3. 1 Summary of test environment, test inputs and purpose of different fatigue test case. 14

Table 5. $1 \mathrm{CPU}$ time and accuracy for 100 randomized iterations while predicting strain for first 50 cycles of ET-F54 test case ................................................................................ 33

Table 5. 2 CPU time and accuracy for 100 randomized iterations while predicting strain for first 50 cycles of ET-F61 test case. 48

Table 9. 1 Summary of the model and experiment data based MCMC simulated probabilistic lives of DMW under different loading and environmental conditions.... 106 
A Hybrid Al/ML and Computational Mechanics Based Approach for Time-Series State and Fatigue Life Estimation of Nuclear Reactor Components

September 2020

ABBREVIATIONS

AI Artificial Intelligence

ANL Argonne National Laboratory

ASME American Society of Mechanical Engineers

DL Deep Learning

DMW Dissimilar Metal Weld

DOE Department of Energy

ET Elevated Temperature

FE Finite Element

HAZ Heat Affected Zone

LAS Low Alloy Steel

LWR Light Water Reactor

LWRS Light Water Reactor Sustainability

ML Machine Learning

PWR Pressurized Water Reactor

ReLU Rectified Linear Unit

SS Stainless Steel 


\section{ACKNOWLEDGMENTS}

This research was supported through the U.S. Department of Energy's Light Water Reactor Sustainability program under the work package of environmental fatigue study with program manager Dr. Thomas. M. Rosseel and deputy program manager Dr. Xiang (Frank) Chen. 


\section{Introduction}

In this report we present data and discussion related to a hybrid predictive modeling framework that can be used for stress analysis and life estimation of nuclear reactor components under cyclic loading. The overall modeling framework is based on limited experiment-data, Artificial-intelligence (AI) Machine-Learning (ML) - Deep-Learning (DL) based techniques and Multiphysics-computationalmechanics based modeling tools. Some of the related theoretical background and results (of the overall modeling framework) are presented in the following sections:

a) Section 2: Brief Theoretical Background of the AI/ML/DL Techniques Used in the Discussed Work

b) Section 3: Loading and Environment of Different Experimental Fatigue Test Cases

c) Section 4: AI/ML Guided Seemingly Strain-Controlled Fatigue Test While Actually Not Controlling the Strain

d) Section 5: Use of AI-Based Machine Learning and Deep Learning Techniques for Time-Series Strain Perdition from Other Sensor Measurements

e) Section 6: A Hybrid AI-TensorFlow and Computational-Mechanics Based Approach for Cyclic Material Parameter Prediction

f) Section 7: Analytical Model Based Cyclic Stress Analysis of Different DMW Test Cases under Constant and Variable Amplitude Cyclic Loading

g) Section 8: Demonstration of the Use of Cyclic-Plasticity Material Parameters in 3D FE Model of a PWR Surge-line Nozzle

h) Section 9: Markov-chain-Monte-Carlo Based Probabilistic-Time-Series State and Fatigue Life Estimation of DMW

i) Section 10: Summary 


\section{Brief Theoretical Background of the Al/ML/DL Techniques Used in the Discussed Work}

In this section, we briefly discuss the theoretical background behind various artificial intelligence (AI), machine learning (ML) and deep learning (DL) techniques used in this report. Open source libraries such as Scikit-learn [1], TensorFlow [2] and Keras [3] were used for simulating the discussed results in this report. Although, these libraries are general purpose and can be used for any predictive modeling/simulation applications, in this report we used these libraries for time-series fatigue data prediction and optimizing the control input for fatigue test cases. The relevant mathematical background only pertinent to the discussed application are briefly discussed below. For more details it is suggested to refer to the websites [1-3] of the above-mentioned libraries and related publications such as [4, 5]. Previous work related to the specific use of the AI/ML technique for time-series fatigue prediction can also be found from [6-16].

\subsection{Justification Behind Using Al/ML/DL Techniques for Fatigue Modeling}

In general, artificial intelligence (AI) techniques are based on set of logic or rules based on which a machine works. Machine learning (ML) and deep learning (DL) techniques are type of generic AI techniques. However, a generic AI technique may or may not produce desired performance/intelligence for a complex nonlinear and unseen problem for which the logic or rules are not previously well defined. Whereas, ML algorithms have the ability to learn without explicitly programming the logic or rules beforehand. A ML algorithm can decipher the structure of a complex nonlinear model if appropriate historical data provided. However, sometime ML algorithm may not give optimal solution due to the complexity of the underlying model. In that case, it might require dataset in additional dimensions (e.g. measurements from other types of sensor) and/or some previous knowledge of the functional form of the underlying model. However, it is not always possible to have dataset in additional dimensions and previous knowledge of the functional form of the underlying model. In that case DL algorithm can be our rescue. Figure 2.1 shows the schematic showing the difference between AI, ML and DL techniques. The DL algorithms are primarily based on multi-layer-perceptron (MLP) based neural network, which was developed based on the inspiration from the biological structure and function of the human brain. Fatigue data e.g. strain versus stress data are highly nonlinear (e.g. refer to Figure 2.2) and often may not be easy to model using conventional physics or mechanics-based approach. The complexity to decipher the underlying model structure further exaggerated by cyclic hardening and cycle softening leading to time-variation of the above mentioned already complex nonlinear system. Overall it leads to a combined nonstationary-nonlinear modeling problem. This type of complex modeling problem is not easy to model, only by using physics-based approach. The recent advancement in AI/ML/DL techniques can be combined with physics-based approach for modeling complex nonstationary-nonlinear models associated with the metal fatigue. 


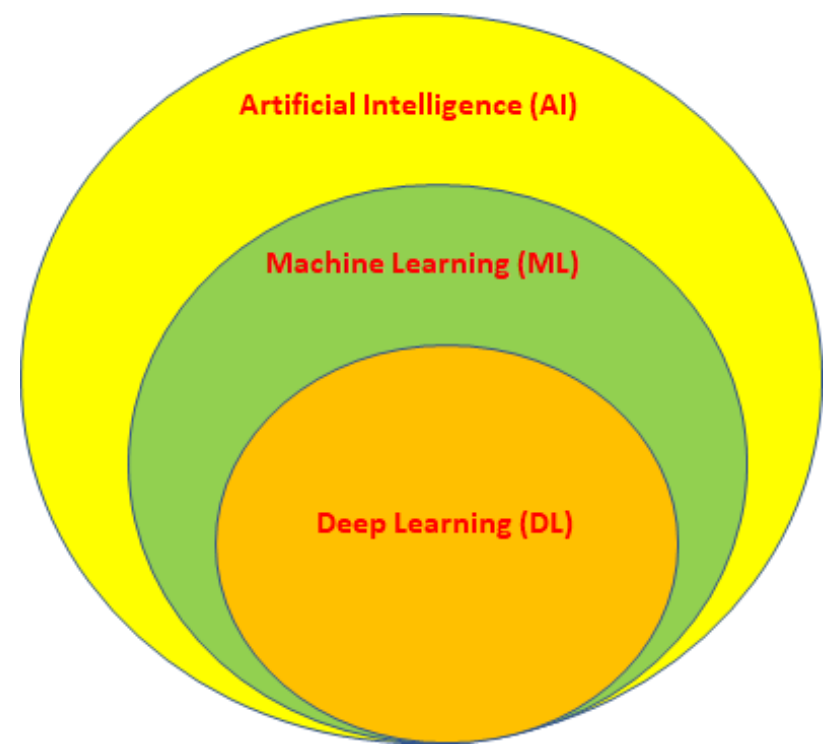

Figure 2. 1 Schematic of the difference between of AI, ML and DL techniques.

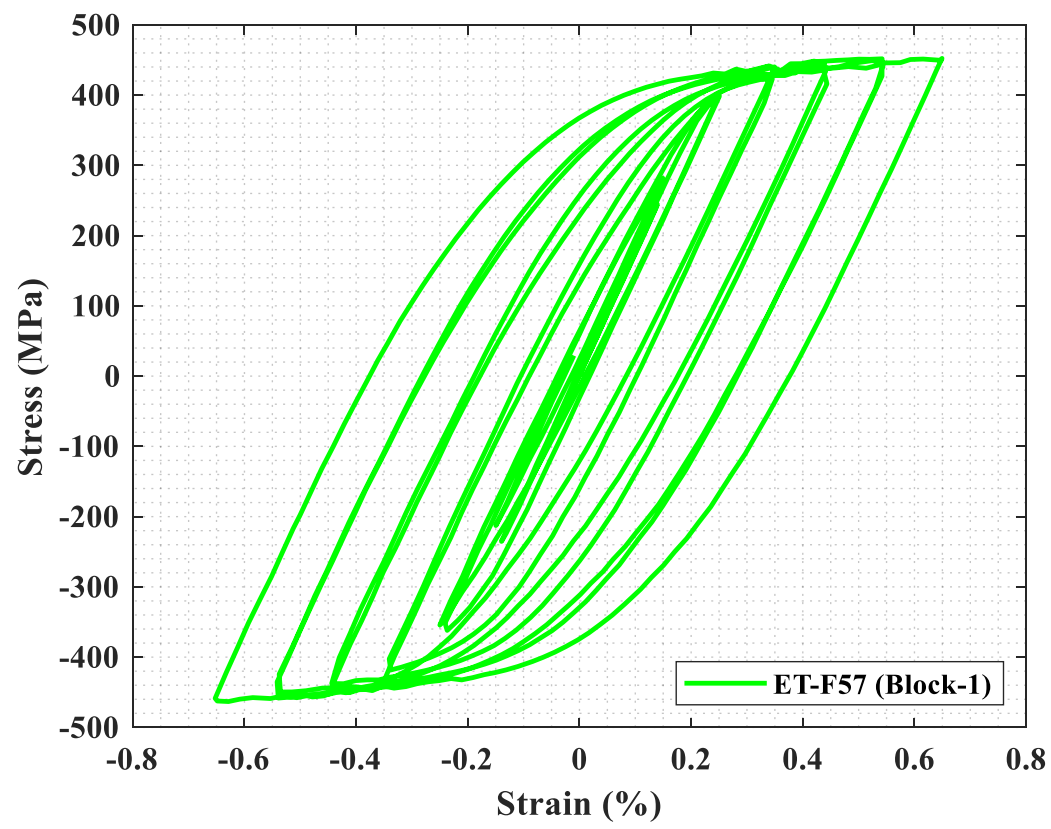

Figure 2. 2 Example strain versus stress hysteresis data for 316SS-508LAS dissimilar metal weld subjected to variable amplitude loading.

\subsection{Brief Introduction to Multi-Layer Perceptron in Context of Time-Dependent Fatigue Data Modeling}

The multi-layer-perceptron (MLP) is an artificial neural network technique consisting of at least 3 layers: one input layer, one output layer and at least one or more hidden or perceptron layers. In an MLP 
network a set of inputs used to train the model. This is by feeding the inputs to the perceptron layer consisting of multiple perceptrons (also known as or node or neurons). Each node/neuron capture a linear function along with an activation function. The outputs of the layer passed into next layer as an input of the next layer. Figure 2.3 shows the schematic of MLP network. The MLP network can be used for modeling time-series fatigue damage. This is by setting a mapping function between the timevarying or cyclic damage causing inputs (e.g. strain, temperature, etc.) and resulting observed outputs (e.g. stress). If we can map the relation between the input-output based on historical fatigue test data, the underlying model can be used for predicting unknown outputs for a given/known set of input information. Before using an MLP network, it must be first trained that means the underlying model structure has to be deciphered. The model training process must be based on historical input-outputs data. The training must be done in an iterative step, with each iterative step consisting of two processes: forward propagation and backward propagation processes (refer to Figure 2.3). In a forward propagation, information feeding process goes from left to right for predicting the output over the entire layers with each layer consisting of multiple nodes/neurons. This is based on chosen weight and biased parameters. In the backward propagation steps, the training process goes from right to left by calculating the partial derivatives of the loss or objective function (L) with respect to different weight and bias parameters of different layers consisting of multiple nodes. In this report mean square error (MSE) based loss function is used to train the Scikit-learn (section 2.3) and Keras (section 2.4) based time-series models. With $y\left(t_{i}\right)$ and $\hat{y}\left(t_{i}\right)$, respectively the true and predicted observation at time $t_{i}$, the MSE based loss function can be given as:

$$
L=\frac{1}{n} \sum_{i=1}^{i=n}\left(y\left(t_{i}\right)-\hat{y}\left(t_{i}\right)\right)^{2}
$$

The backward process is to update the previously chosen/calculated weight and bias parameters of each layer so that the loss function (which track the error in each iteration) is reduces in the next iteration. The eventual aim is to find the global optima of weight and bias parameters. Finding global optima sometime is not straight forward, often complexity of the model can lead to local optima trap. Figure 2.4 shows the difference between global optima and local optima trap. Time-series fatigue modeling can often lead to non-convex function modeling problem associated with local optima trap. This is due to nonstationary fatigue damage process associated with cyclic hardening/softening and its association with multiple failure/damage modes. A nonstationary damage process can be modeled using piecewise time-series modeling which results are discussed in section 5. 


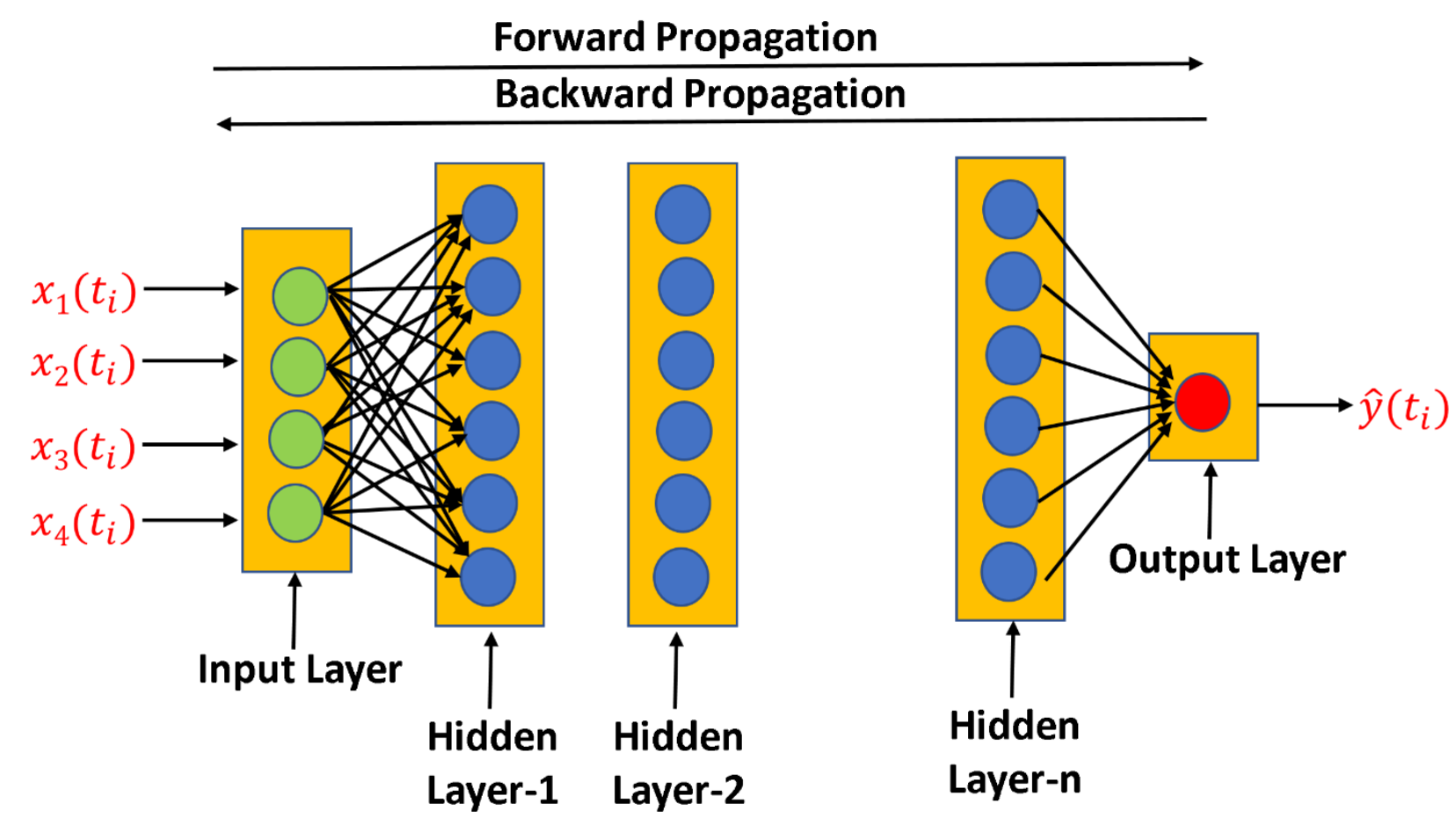

Figure 2. 3 Schematic of an MLP network with time-varying fatigue damage causing inputs (e.g. strain, temperature, etc.) and resulting observed outputs (e.g. stress).

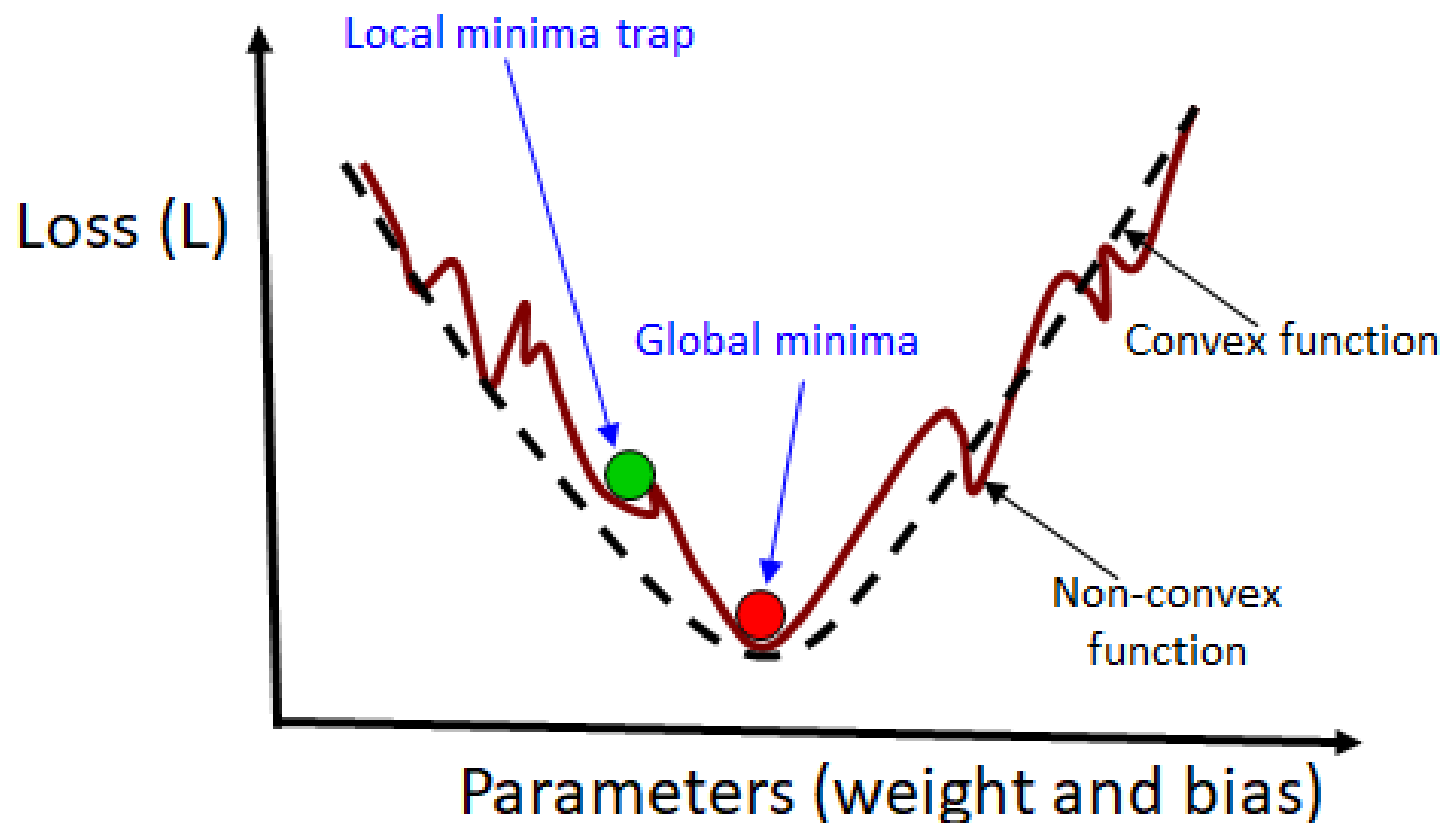

Figure 2. 4 Schematic showing the difference between global optima and local optima trap. 


\subsection{Use of Scikit-learn-Regression Library for Time-Series Fatigue Data Prediction}

The Scikit-learn library [1] was used for predicting time-series strain from other sensor measurements. The related results are presented in section 5. The Scikit-learn (also popularly known as sklearn) library is a python-based AI/ML library that can used for time-series regression modeling problems. In the discussed work (refer to section 5) Scikit-learn based multivariable regression technique was used to predict unmeasurable strains in a PWR-water system. In a PWR-water system (refer to Figure 3.6), the test specimen is required to be placed in a narrow autoclave in which an extensometer cannot be placed to measure strains due to lack of space, wiring problems and due to hightemperature flow inside the autoclave). This led us to use AI/ML based Scikit-learn/regression technique for predicting the time-series strains based on the other sensor measurements. For given set of historical data such as from a replica in-air test (refer to Figure 3.5), an input-output mapping function can be estimated. Note that in an open in-air test system, strain can be measured by placing an extensometer at the gauge section of the specimen. Based on the in-air-test-based mapping function, the time-dependent strain in an autoclave environment can be estimated for a given set of time-dependent input variables (e.g. sensor readings measured in PWR-water system). For the mentioned multivariate regression problem four independent dimensions were considered. Those are time, displacement measurements through pull-rod, stress measurements through load-cell, and actuator-displacement measurements through LVDT sensors. The time-dependent strain can be expressed in terms of these input dimensions $\left(x_{i=1, . .4}\right)$ and associated model parameters $\left(\theta_{i=0, . .4}\right)$ as follows:

$$
\hat{\epsilon}\left(t_{i}\right)=\theta_{0}+\theta_{1} x_{1}\left(t_{i}\right)+\theta_{2} x_{2}\left(t_{i}\right)+\theta_{3} x_{3}\left(t_{i}\right)+\theta_{4} x_{4}\left(t_{i}\right)
$$

Given the set of historical $x\left(t_{i}\right)=\left\{x_{1}\left(t_{i}\right), x_{2}\left(t_{i}\right), x_{3}\left(t_{i}\right), x_{4}\left(t_{i}\right)\right\}$ and true value of strain $\epsilon\left(t_{i}\right)$ the model parameters $\left(\theta_{i=0, . .4}\right)$ can be estimated by optimizing the following loss function:

$$
L=\frac{1}{n} \sum_{i=1}^{i=n}\left(\epsilon\left(t_{i}\right)-\hat{\epsilon}\left(t_{i}\right)\right)^{2}
$$

As discussed in section 2.2, a global optimum of $\theta_{i=0, . .4}$ may not be achievable for the entire fatigue life requiring piecewise parameter estimation. The piecewise parameter estimation can be done by clustering/grouping the entire fatigue life to different sets. The clustering can be done using Scikit-learn library-based k-mean clustering technique discussed in section 2.6.

\subsection{Use of Keras Deep Learning Library for Time-Series Fatigue Data Prediction}

The Keras library [3] was used for predicting strain from other sensor measurements and the related results are presented in section 5. As mentioned in section 2.1 it may not always possible to predict a nonstationary-nonlinear function accurately using a usual AI/ML based technique (such as above discussed Scikit-learn based techniques), unless data from all underlying dimensions and/or the functional structure of the mapping function is known beforehand. Although for predicting strain from 
other sensor measurement, we used the above mentioned Scikit-learn based AI/ML library, we tried to further improve the accuracy by using deep learning based Keras library. This library is a high-level application programming interface (API) and based on the MLP network discussed in section 2.2. The high-level API of Keras is based on underlying TensorFlow library [2], which brief discussion follows. Figure 2.5 shows the relation between Keras and TensorFlow library. To estimate the mapping function between strain and input dimensions (refer to section 2.3), three perceptron layers with each layer having ten neurons (refer to section 2.2) were used. The parameters were optimized using the loss function given in Eq. 2.3. The Keras models were tested using both Rectified Linear Unit (ReLU) and Sigmoid activation functions [2,3].

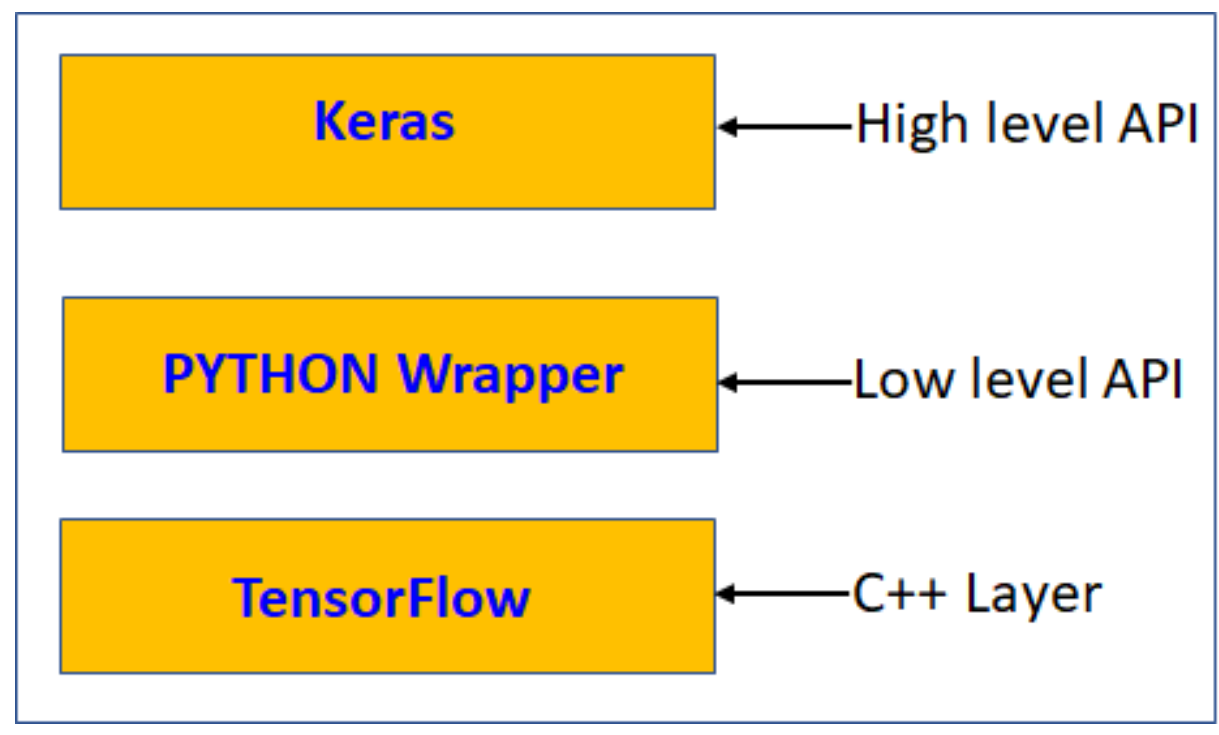

Figure 2. 5 Schematic showing the relation between Keras and TensorFlow library.

\subsection{Use of TensorFlow Library for Cyclic Stress-Strain Curve Prediction}

The TensorFlow (TF) library [2] was used for predicting equivalent cyclic stress-strain curve from the variable-amplitude test-based stress-strain data. The related results are presented in section 6 . The TensorFlow library is based on graph-based computation. Compare to Keras library, TF library gives flexibility to choose appropriate functional form for faster convergence compared to the higher level Keras library. However, one need to provide appropriate function. We selected hyperbolic tangent function to map the underlying strain versus stress data (for example refer to Figure 2.2) measured under a single loading block (refer to Figure 3.10). The predicted stress $\left(\hat{\sigma}\left(t_{i}\right)\right)$ of the cyclic-stress-strain curve can be represented in terms of the input strain $\varepsilon\left(t_{i}\right)$ using the following expression:

$$
\hat{\sigma}\left(t_{i}\right)=W * \tanh \left(\varepsilon\left(t_{i}\right)\right)+b
$$

where $W$ and $b$ are the weight and bias parameters (if any), respectively. The resulting equivalent stressstrain curve can be estimated by optimizing the following loss function: 


$$
L=\frac{1}{n} \sum_{i=1}^{i=n}\left(\sigma\left(t_{i}\right)-\hat{\sigma}\left(t_{i}\right)\right)^{2}
$$

The loss or objective function can be optimized by using the gradient tape API within the TF library. The gradient tape API library perform the automated partial differentiation of the loss function.

\subsection{Use of Scikit-learn Library Based k-Mean Clustering Technique for Optimizing Fatigue-Test Control Inputs and Improving Time-Series Fatigue Data Prediction Techniques}

The Scikit-learn library [1] based k-mean clustering technique was used for optimizing the inputs for pull-rod-displacement-control-based PWR-water fatigue test cases (refer to section 4). The k-mean clustering technique was also used for dividing the entire fatigue life to different subdomains for piecewise strain prediction (refer to section 5). The k-mean clustering is a method of vector quantization used for segregating a given multi-dimensional vector data set in to ' $k$ ' numbers of cluster. This is by choosing initial cluster centers and then optimizing the locations of those cluster centers by optimizing the euclidean distance between the cluster centers and the given multi-dimensional ' $n$ ' data points. Figure 2.6 shows an example two-dimensional data sets with four cluster (and cluster center). Whereas, a m-dimensional fatigue data set can be represented by the following tensor:

$$
\boldsymbol{X}=\left[\boldsymbol{x}_{1}\left(t_{i}\right), \boldsymbol{x}_{2}\left(t_{i}\right), \boldsymbol{x}_{3}\left(t_{i}\right) \ldots \ldots . \boldsymbol{x}_{\boldsymbol{m}}\left(t_{i}\right)\right]
$$

Where, $\boldsymbol{x}_{j=1,2 \ldots m}$ are individual vector data representing different underlying dimensions with each dimension consisting of $t_{i-1,2 \ldots n}$ time-dependent data. For the discussed fatigue modeling case the cycle/time dependent maximum/minimum stress, pull-rod displacement, stress and/or frame actuator displacement are used as input dimensions. Assuming there are ' $\mathrm{k}$ ' numbers of cluster, the centroid of the individual cluster can be a function of underling input dimensions (or coordinates) and can be expressed as:

$$
\mu^{p=1,2 \ldots k}=\left\{x_{1}^{p}, x_{2}^{p}, x_{3}^{p}, \ldots \ldots . x_{m}^{p}\right\}
$$

The euclidean distance between the $\mathrm{p}^{\text {th }}$ cluster center and different $\mathrm{m}$-dimensional data points can be expressed as:

$$
d^{p=1,2 \ldots k}=\sqrt{\left(x_{1}\left(t_{1}\right)-x_{1}^{p}\right)^{2}+\left(x_{2}\left(t_{1}\right)-x_{2}^{p}\right)^{2}+\cdots\left(x_{1}\left(t_{2}\right)-x_{1}^{p}\right)^{2}+\left(x_{2}\left(t_{2}\right)-x_{2}^{p}\right)^{2}+\cdots}
$$


The optimal k-mean cluster can be estimated by optimizing the loss or objective function such as sum of the square error between individual data points and the iteratively optimized cluster centers. The loss function can be given as:

$$
L=\sum_{p=1}^{k} \sum_{i=1}^{i=n} d\left(\boldsymbol{x}_{j}, \mu^{p}\right)^{2}
$$

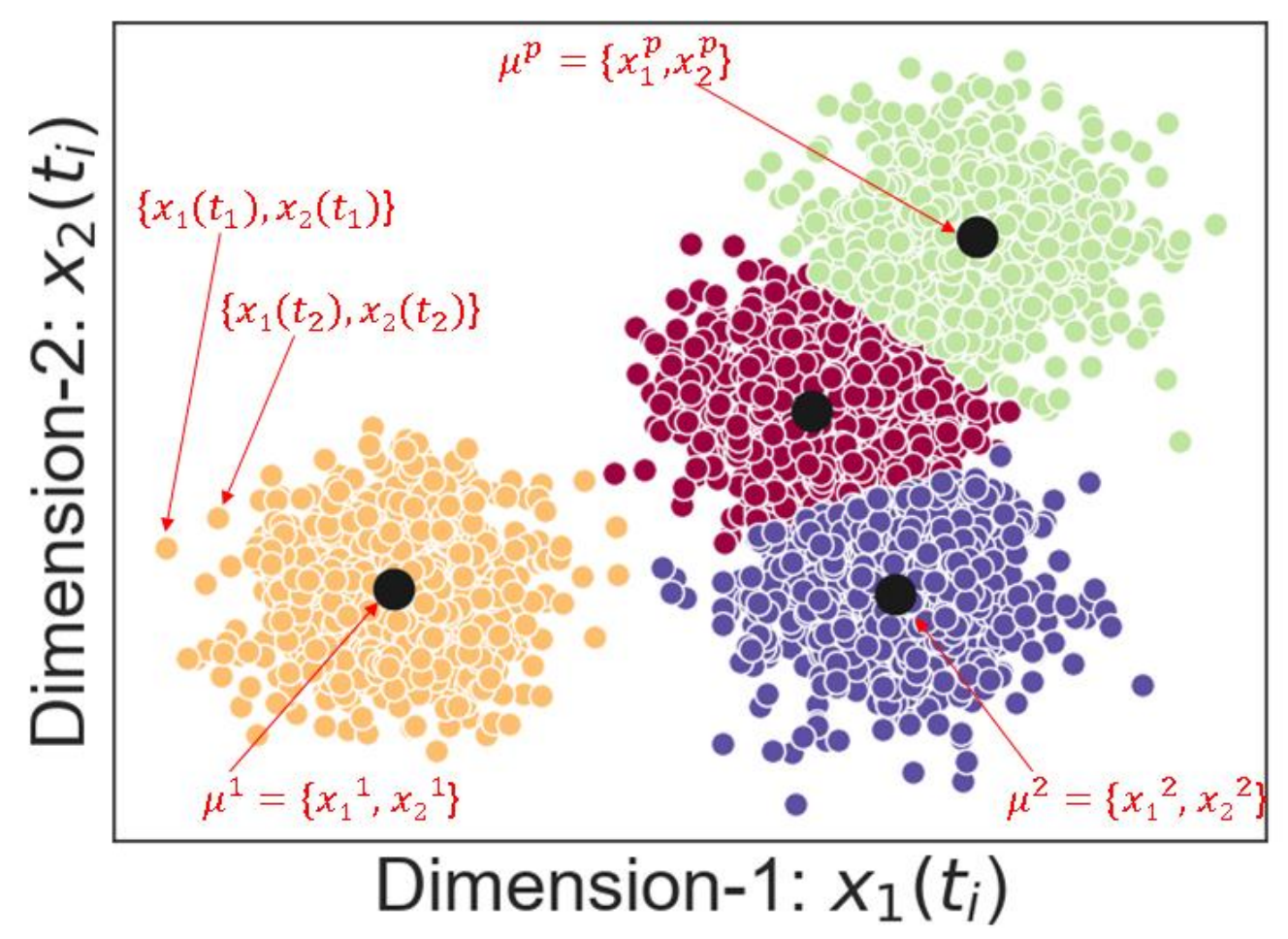

Figure 2. 6 Example two-dimensional data sets with four cluster.

\subsection{Markov-Chain-Monte-Carlo Based Probabilistic Fatigue Life Prediction}

In our previous work [6] we proposed the use of Markov-Chain-Monte-Carlo (MCMC) techniques for probabilistic prediction of time-dependent damage states (such as stress). Then, the probabilistic fatigue life can be estimated based on the estimated probabilistic time-series data and given a failure criterion. In section 9 we used the MCMC model for probabilistic estimation of the cycle versus maximum stress data for DMW test cases. Based on this probabilistic-time-series data and given failure criteria (such as ASME 25\% load drop criteria) the probabilistic life of DMW test cases was estimated. 


\section{Loading and Environment of Different Experimental Fatigue Test Cases}

In this section different fatigue test cases and their purpose are summarized. The related strain amplitudes, specimen dimension, material and test environments are presented. According to their use, the associated test results are presented and discussed in the succeeding sections.

\subsection{Justification for Selecting Strain Amplitudes for Different Fatigue Testing}

In our previous report [17], we presented detailed results and discussion associated with 3D finite element (FE) model based thermal-mechanical stress analysis of PWR surge-line nozzle and pressurizerbottom-head assembly. This model was simulated under design-basis loading (with considering corresponding temperature and pressure transients under the entire heat-up, full-power-operation and cool-down operations). The model was simulated considering most limiting displacement boundary conditions (for details refer to [17]). Below presented Figures 3.1 and 3.2 show some representative results (directly taken from [17]) based on which the strain amplitude of 0.6 to $0.65 \%$ are selected for majority of the test cases discussed in this report. For example, Figure 3.1 shows the FE simulated total, thermal, and mechanical strain (along max. principal direction) at the maximum stress element of the DMW region (refer to Figure 3.2) of the surge-line nozzle assembly. From the Figure 3.1 the DMW filer weld region experiences a maximum principal thermal, mechanical and total strains of $0.3426 \%, 1.0642$ $\%$ and $1.4068 \%$, respectively. From the Figure 3.2, DMW filler weld region experiences lowest thermal strain compared to the other regions. Despite of lower thermal strain, the DMW filler weld regions experiences a high mechanical and total strain. In a severe limiting (thermal and displacement) boundary condition case, a high total and mechanical strain can lead to low cycle fatigue failure of surge-line nozzle. Based on the above-mentioned maximum mechanical and total strain range of $1.0642 \%$ and $1.4068 \%$ respectively, we selected a maximum strain amplitude of either 0.6 or $0.65 \%$ for most of the discussed test cases. This is in assumption that the developed material model can capture the stressstrain behavior of surge-line nozzle subjected to thermal-mechanical loading that can create a maximum strain range of 1 to $1.4 \%$. 

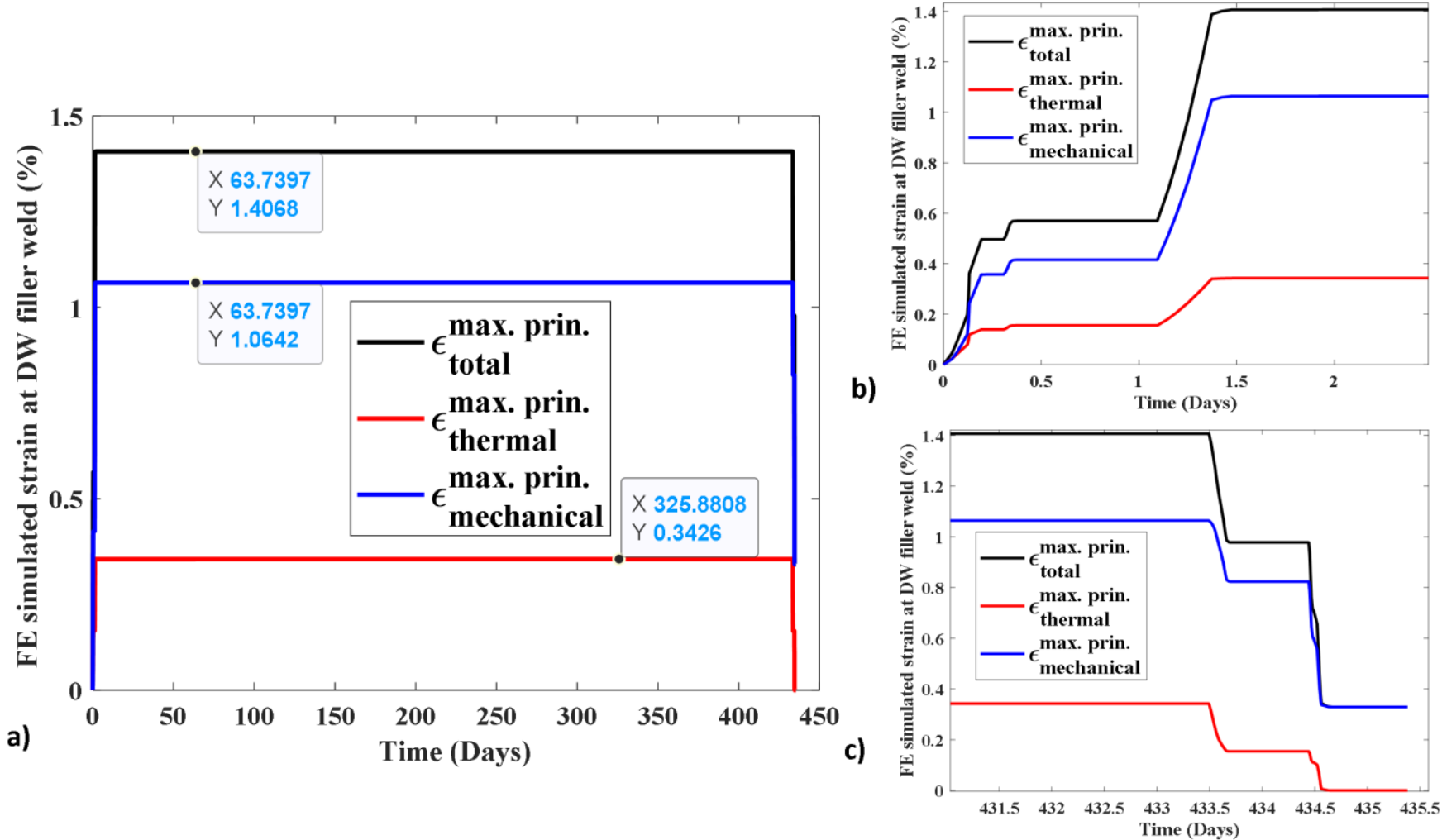

Figure 3. 1 FE model (case-1) simulated total, thermal, and mechanical strain (along max. principal direction) at the maximum stress element of the DMW-filler: (a) full design-basis cycle, (b) during heatup, and (c) during cool-down [17]

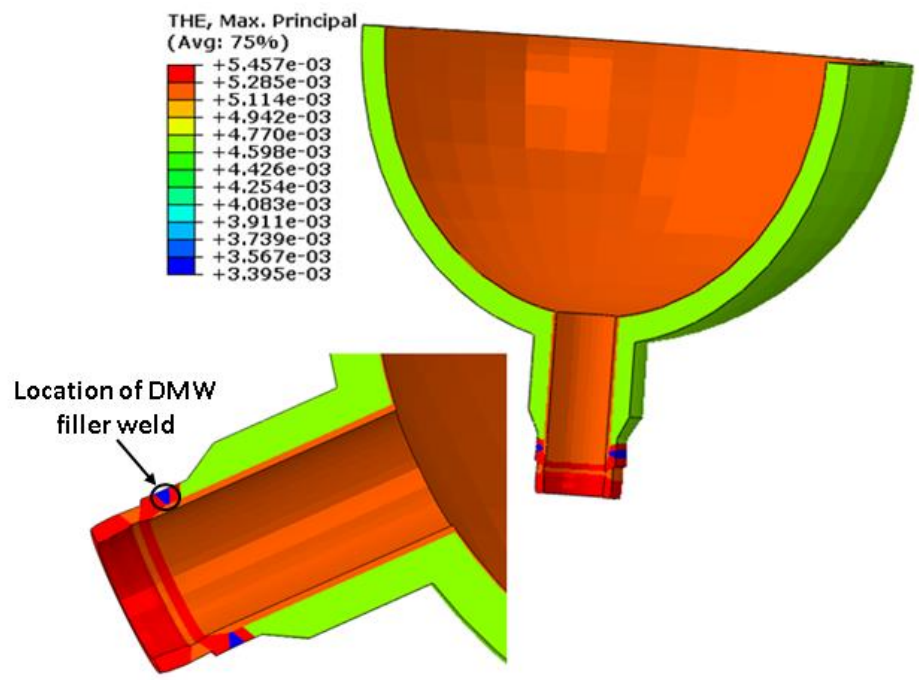

Figure 3. 2 Maximum principal thermal strain contour plot at 1.3717 days (32.921 hours) from the start of the heat-up operation showing the DMW region experiences least thermal strain compared to other regions [17]. 


\subsection{Test Specimen and Test Setup}

In this report, we present result from fatigue tests either conducted using 316ss pure base metal specimens or DMW filler/butter weld specimens. Figure 3.3 shows the cross-section of the weld plates (only near the weld region) from which the weld specimens were fabricated. The fatigue tests were conducted using the weld specimens. For all test we used hourglass-type specimens, which geometry information can be found from Figure 3.4. We used either in-air test system (Figure 3.5) or a PWRwater-loop test system (Figure 3.6) to conduct the respective in-air or PWR-water environment fatigue tests.

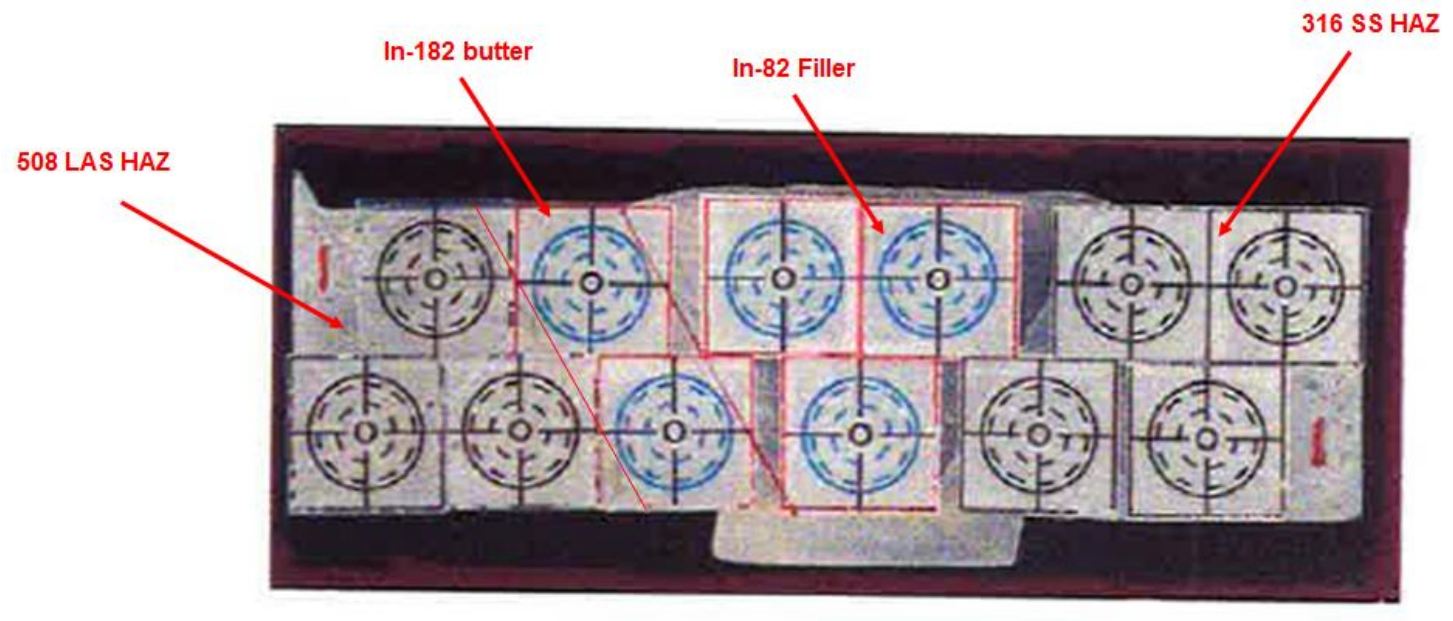

Figure 3. 3 Cross-section of the weld plates (only near the weld region) and location of the along-thelength weld and HAZ specimens.

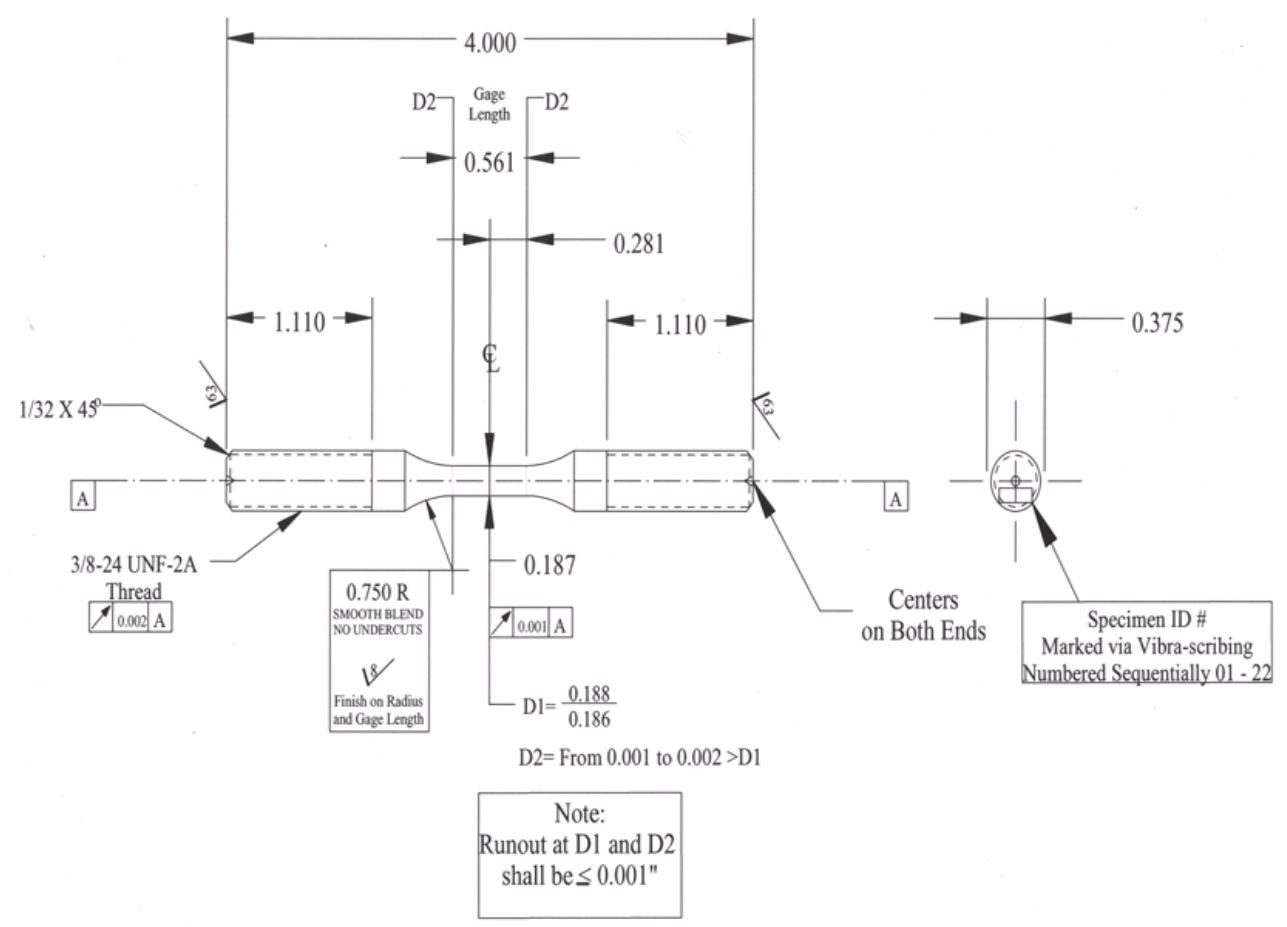

Figure 3. 4 Geometry of hourglass-type specimens. 


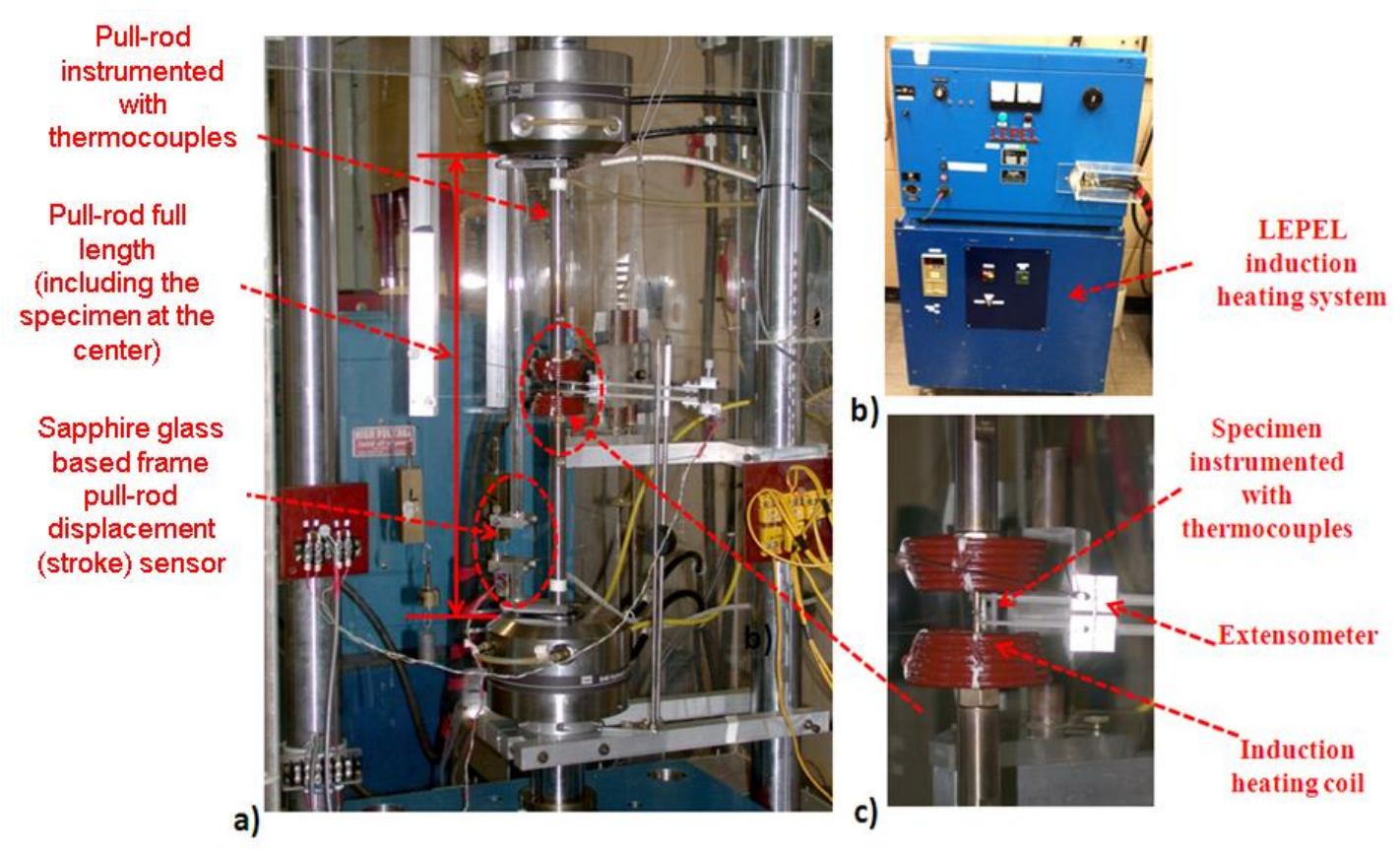

Figure 3. 5 a) Test section with induction heating coil, b) LEPEL induction heating system, and c) close view of induction heating coil and specimen and high-temperature extensometer location.

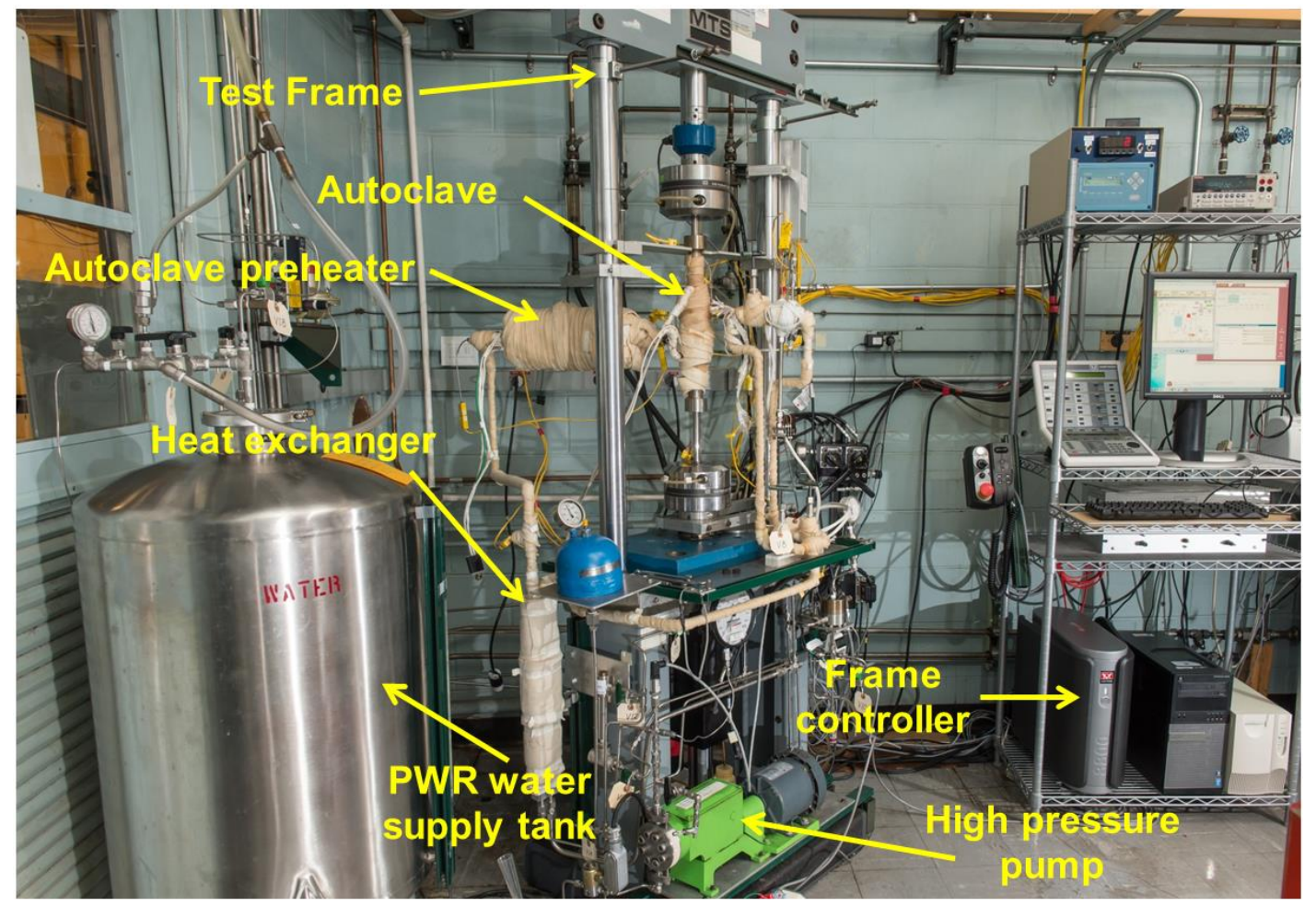

Figure 3. 6 Environmental test loop showing different subsystems. 


\subsection{Loading Inputs and Environment for Different Test Cases}

All the tests reported in this report were conducted in an intention to create certain strain amplitudes in the gauge area of the test specimen. However, the tests were conducted either controlling the gauge area strain or the pull-rod displacement (refer to Figure 3.5). Table 3.1 summarizes the test environment, intended strain amplitude, strain rates and the control mode followed for the individual test cases. This table also summarizes the purpose of the different test cases in context of the results discussed in this report. The input loading for the different test cases are presented through Figure 3.7 to 3.14. Other than the AI-guided multistep test cases (ET-F58, ET-F61 and EN-F62), the shown test inputs were repeated for the entire fatigue life. For AI-guided multistep test cases the loading inputs were presented for first cycle only as an example. However for AI-guided test cases, different set of test inputs were used for different groups of cycles. The grouping of cycles was based on cluster analysis results discussed in section 4.

Table 3. 1 Summary of test environment, test inputs and purpose of different fatigue test case.

\begin{tabular}{|c|c|c|c|c|}
\hline Test ID & Material & $\begin{array}{c}\text { Test } \\
\text { environment }\end{array}$ & Test input & Purpose in this report \\
\hline $\begin{array}{c}\text { *LWRS/ } \\
\text { ET-F13 }\end{array}$ & $\begin{array}{c}316 \text { base } \\
\text { metal }\end{array}$ & $\begin{array}{l}\text { In-air, } \\
300{ }^{\circ} \mathrm{C}\end{array}$ & $\begin{array}{l}\text { Non- } \\
\text { optimized pull-rod } \\
\text { displacement } \\
\text { (max. amp of } \\
0.1313 \text { mm } \text { or } \\
5.1693 \quad \text { mil) } \\
\text { control } \\
\text { intended with } \\
\text { strain amp. } 0.5 \% \\
\text { and strain rate of } \\
0.1 \% / \mathrm{s}\end{array}$ & $\begin{array}{l}\text { \# Comparison w.r.t AI/ML } \\
\text { guided pseudo-strain-controlled } \\
\text { fatigue test (refer to section 4) }\end{array}$ \\
\hline $\begin{array}{c}\text { *LWRS/ } \\
\text { ET-F49 }\end{array}$ & $\begin{array}{c}316 \text { base } \\
\text { metal }\end{array}$ & $\begin{array}{l}\text { In-air, } \\
300^{\circ} \mathrm{C}\end{array}$ & $\begin{array}{c}\text { Improved but not } \\
\text { optimized pull-rod } \\
\text { displacement } \\
\text { control with } \\
\text { intended max. } \\
\text { strain amp. } 0.65 \% \\
\text { and strain rate of } \\
0.1 \% / \mathrm{s}\end{array}$ & $\begin{array}{l}\text { \# Comparison w.r.t AI/ML } \\
\text { guided pseudo-strain-controlled } \\
\text { fatigue test (refer to section 4) }\end{array}$ \\
\hline $\begin{array}{l}\text { *LWRS/ } \\
\text { ET-F54 }\end{array}$ & $\begin{array}{l}82 / 182 \\
\text { DMW } \\
\text { (Filler } \\
\text { weld) }\end{array}$ & $\begin{array}{l}\text { In-air, } \\
300{ }^{\circ} \mathrm{C}\end{array}$ & $\begin{array}{l}\text { Non-optimized } \\
\text { pull-rod } \\
\text { displacement } \\
\text { control based on } \\
\text { FE model of PWR } \\
\text { SL nozzle under } \\
\text { design basis load } \\
\text { (with intended } \\
\text { max. strain range }\end{array}$ & $\begin{array}{l}\text { \# AI/ML based strain prediction } \\
\text { validation under non-stationary } \\
\text { type loading } \\
\text { (refer to section } 5 \text { ) }\end{array}$ \\
\hline
\end{tabular}




\begin{tabular}{|c|c|c|c|c|}
\hline & & & $\begin{array}{c}\text { of } 0.91 \% \text { and } \\
\text { strain rate of } \\
0.1 \% / \mathrm{s})\end{array}$ & \\
\hline $\begin{array}{l}\text { LWRS/ } \\
\text { ET-F57 }\end{array}$ & $\begin{array}{l}82 / 182 \\
\text { DMW } \\
\text { (Filler } \\
\text { weld) }\end{array}$ & $\begin{array}{l}\text { In-air, } \\
300{ }^{\circ} \mathrm{C}\end{array}$ & $\begin{array}{l}\text { Variable amplitude } \\
(0.1 \text { to } 0.65 \%) \\
\text { strain control with } \\
\text { strain rate of } \\
0.01 \%\end{array}$ & $\begin{array}{l}\text { \# Hybrid AI/ML and solid } \\
\text { mechanics-based } \\
\text { material/constitutive model } \\
\text { development (refer to section 6) } \\
\text { \# Validation of computational } \\
\text { mechanics-based analytical } \\
\text { model (refer to section 7) } \\
\text { \# Markov-Chain-Monte-Carlo } \\
\text { model for probabilistic life } \\
\text { estimation (refer to section 9) }\end{array}$ \\
\hline $\begin{array}{l}\text { LWRS/ } \\
\text { ET-F58 }\end{array}$ & $\begin{array}{c}316 \text { base } \\
\text { metal }\end{array}$ & $\begin{array}{l}\text { In-air, } \\
300{ }^{\circ} \mathrm{C}\end{array}$ & $\begin{array}{c}\text { AI/ML based } \\
\text { optimized pull-rod } \\
\text { displacement } \\
\text { control with } \\
\text { intended max. } \\
\text { strain amp. } 0.6 \% \\
\text { and strain rate of } \\
0.01 \% / \mathrm{s}\end{array}$ & $\begin{array}{l}\text { \# Validation of AI/ML guided } \\
\text { pseudo-strain-controlled fatigue } \\
\text { test (refer to section } 4 \text { ) }\end{array}$ \\
\hline $\begin{array}{l}\text { LWRS/ } \\
\text { ET-F59 }\end{array}$ & $\begin{array}{l}\text { 82/182 } \\
\text { DMW } \\
\text { (Butter } \\
\text { weld) }\end{array}$ & $\begin{array}{l}\text { In-air, } \\
300{ }^{\circ} \mathrm{C}\end{array}$ & $\begin{array}{c}\text { Constant } \\
\text { amplitude }(0.6 \%) \\
\text { strain control with } \\
\text { strain rate of } \\
0.01 \% / \mathrm{s}\end{array}$ & $\begin{array}{l}\text { \# Comparison w.r.t AI/ML } \\
\text { guided pseudo-strain-controlled } \\
\text { fatigue test (refer to section 4) } \\
\text { \# Validation of computational } \\
\text { mechanics-based analytical } \\
\text { model (refer to section 7) }\end{array}$ \\
\hline $\begin{array}{l}\text { LWRS/ } \\
\text { ET-F60 }\end{array}$ & $\begin{array}{l}82 / 182 \\
\text { DMW } \\
\text { (Filler } \\
\text { weld) }\end{array}$ & $\begin{array}{l}\text { In-air, } \\
300{ }^{\circ} \mathrm{C}\end{array}$ & $\begin{array}{l}\text { Non- optimized } \\
\text { pull-rod } \\
\text { displacement } \\
\text { control with } \\
\text { intended max. } \\
\text { strain amp. } 0.6 \% \\
\text { and strain rate of } \\
0.01 \% / \mathrm{s} \\
\end{array}$ & $\begin{array}{l}\text { \# Comparison w.r.t AI/ML } \\
\text { guided pseudo-strain-controlled } \\
\text { fatigue test (refer to section 4) } \\
\text { \# Validation of computational } \\
\text { mechanics-based analytical } \\
\text { model (refer to section 7) }\end{array}$ \\
\hline $\begin{array}{l}\text { LWRS/ } \\
\text { ET-F61 }\end{array}$ & $\begin{array}{l}82 / 182 \\
\text { DMW } \\
\text { (Filler } \\
\text { weld) }\end{array}$ & $\begin{array}{l}\text { In-air, } \\
300{ }^{\circ} \mathrm{C}\end{array}$ & $\begin{array}{c}\text { AI/ML based } \\
\text { optimized pull-rod } \\
\text { displacement } \\
\text { control with } \\
\text { intended max. }\end{array}$ & $\begin{array}{l}\text { \# Validation of AI/ML guided } \\
\text { pseudo-strain-controlled fatigue } \\
\text { test (refer to section } 4 \text { ) } \\
\text { \# AI/ML based strain prediction }\end{array}$ \\
\hline
\end{tabular}




\begin{tabular}{|c|c|c|c|c|}
\hline & & & $\begin{array}{c}\text { strain amp. } 0.6 \% \\
\text { and strain rate of } \\
0.01 \% / \mathrm{s}\end{array}$ & $\begin{array}{l}\text { validation under non-stationary } \\
\text { type loading } \\
\text { (refer to section 5) } \\
\text { \# Validation of computational } \\
\text { mechanics-based analytical } \\
\text { model (refer to section 7) }\end{array}$ \\
\hline $\begin{array}{l}\text { LWRS/ } \\
\text { EN-F62 }\end{array}$ & $\begin{array}{l}\text { 82/182 } \\
\text { DMW } \\
\text { (Filler } \\
\text { weld) }\end{array}$ & $\begin{array}{c}\text { PWR-Water, } \\
300^{\circ} \mathrm{C} \text {, Water } \\
\text { chemistry: } \\
1000 \mathrm{ppm} \mathrm{B} \\
\text { as } \mathrm{H}_{3} \mathrm{BO}_{3}, 2 \\
\text { ppm Li } \mathrm{Li}^{+} \text {as } \\
\mathrm{LiOH}, 20 \% \\
\mathrm{H}_{2} / \mathrm{bal} . \mathrm{N}_{2} \\
\text { cover gas, } \\
\text { and } \mathrm{DO}<5 \\
\text { ppb }\end{array}$ & $\begin{array}{c}\text { AI/ML based } \\
\text { optimized pull-rod } \\
\text { displacement } \\
\text { control with } \\
\text { intended max. } \\
\text { strain amp. } 0.6 \% \\
\text { and strain rate of } \\
0.01 \% / \mathrm{s}\end{array}$ & $\begin{array}{l}\text { \# AI/ML based strain prediction } \\
\text { under non-stationary type loading } \\
\text { and PWR-water environment } \\
\text { (refer to section 5) } \\
\text { \# PWR water condition Fen } \\
\text { estimation of 82/182 DMW } \\
\text { \# Markov-Chain-Monte-Carlo } \\
\text { model for probabilistic life } \\
\text { estimation of DMW } \\
\text { (refer to section 9) }\end{array}$ \\
\hline
\end{tabular}

* Tests were conducted in earlier FY and some of the related results can be found from our earlier publications [17].

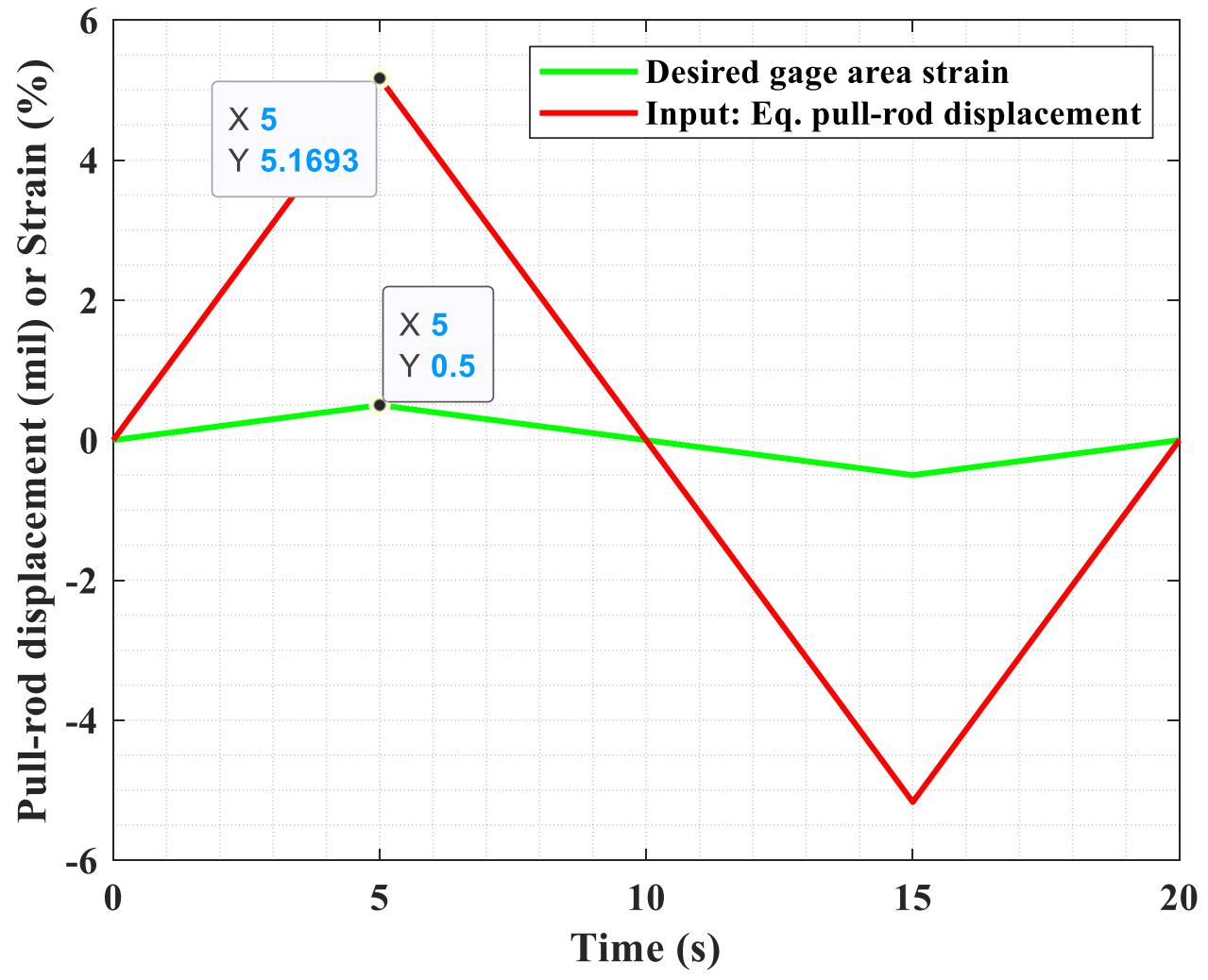

Figure 3. 7 Pull-rod-displacement that was applied to ET-F13 (316SS base) specimen until its failure. 


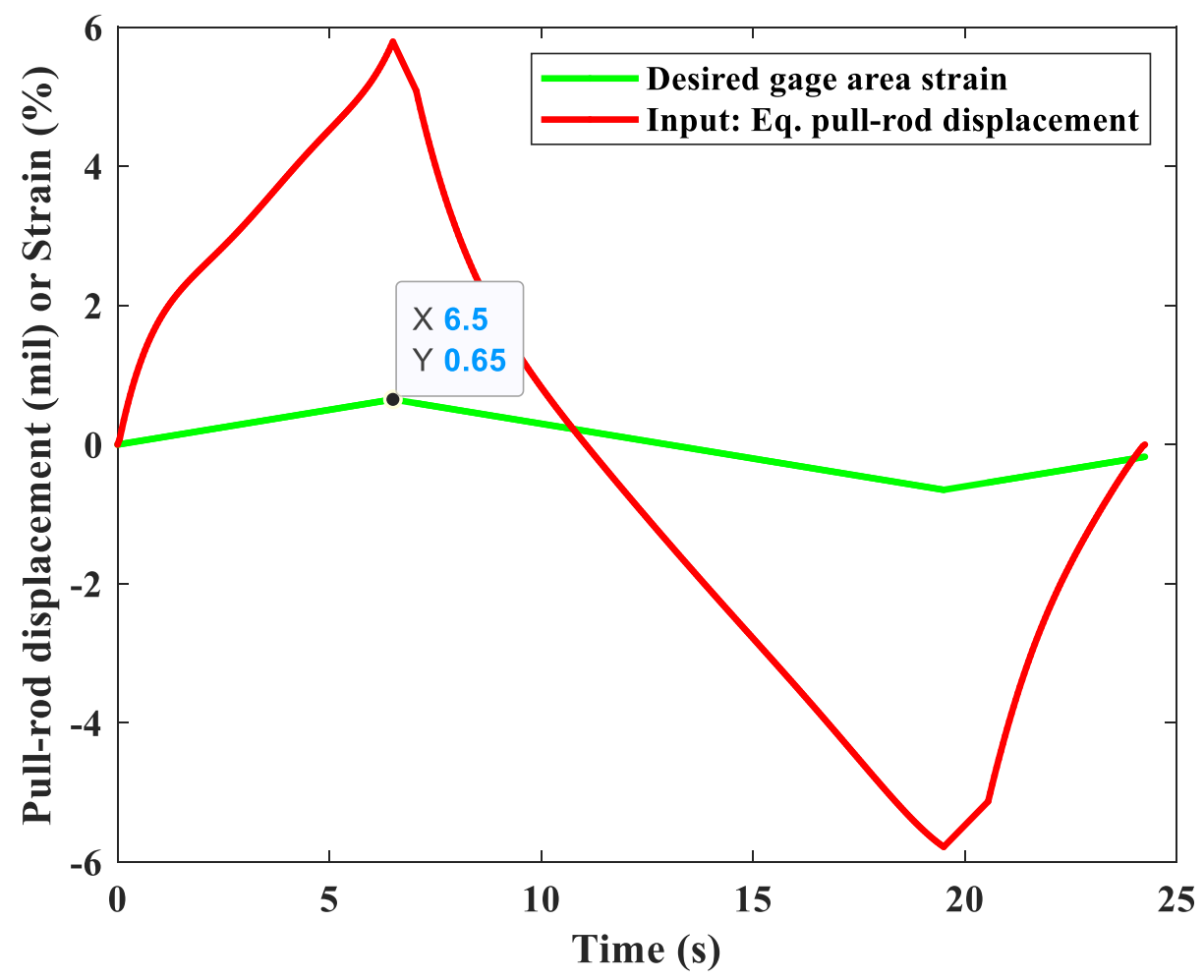

Figure 3. 8 Improved pull-rod-displacement (and corresponding intended strain amplitude) that was applied to ET-F49 (316SS base) specimen until its failure.

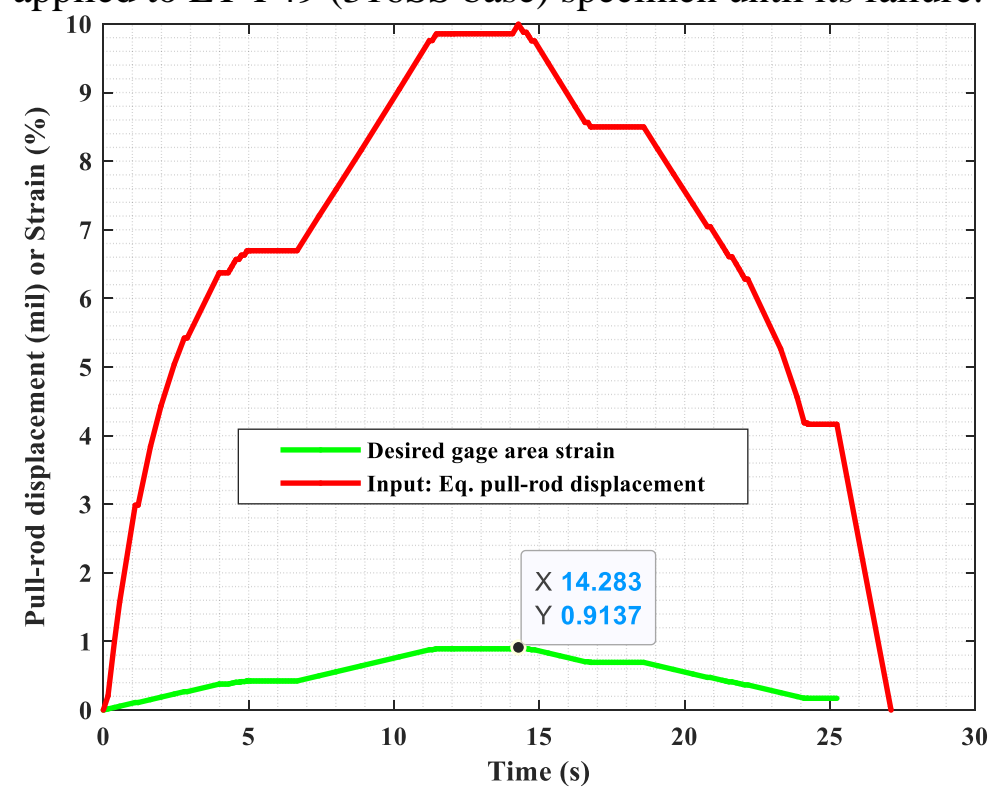

Figure 3. 9 Pull-rod displacement (and corresponding intended strain amplitude) that was applied to ETF54 (82/182 DMW filler weld) specimen over the entire fatigue life. 


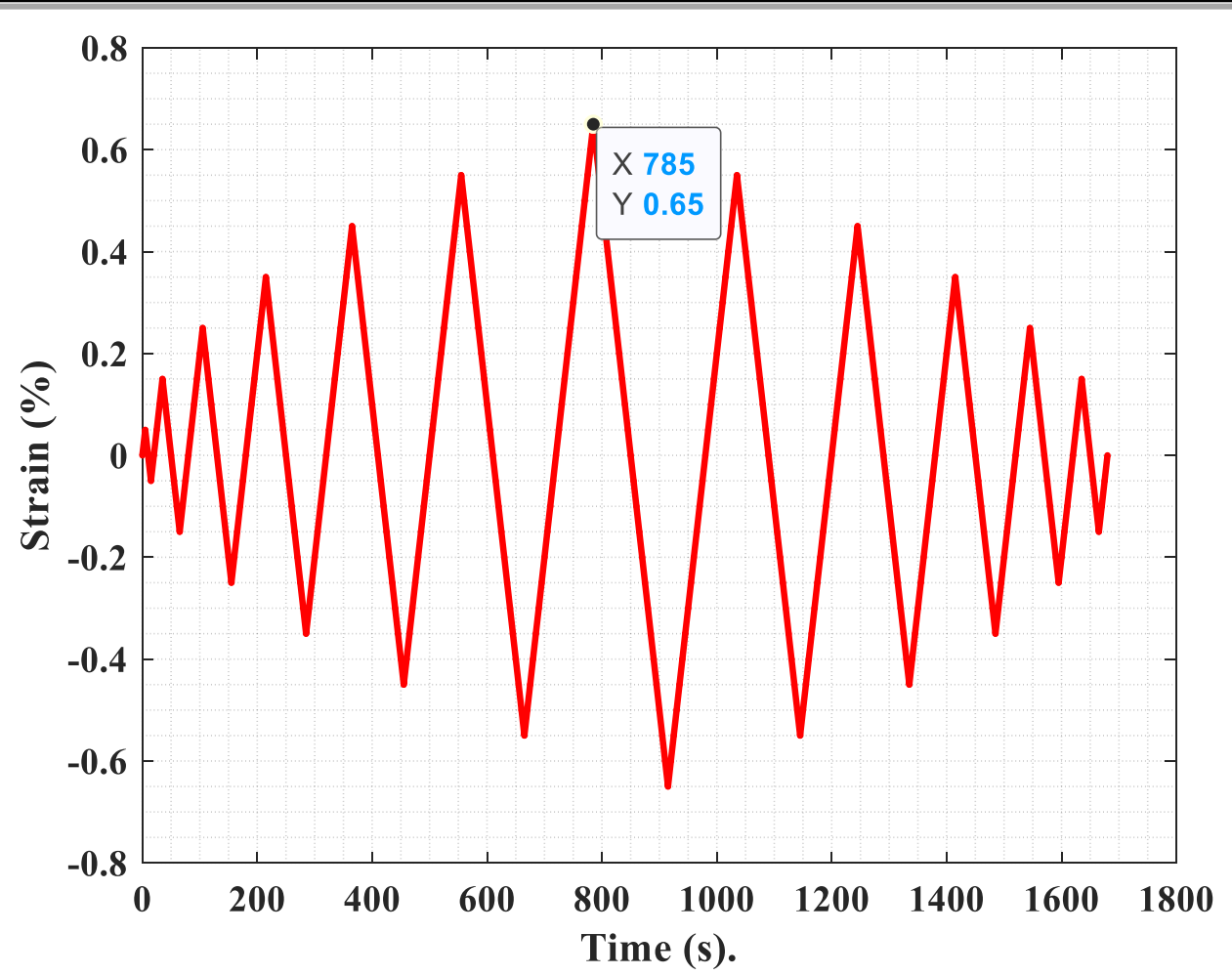

Figure 3. 10 A single-block of variable amplitude gage-area strain that was applied to ET-F57 (82/182 DMW filler weld) specimen until its failure.

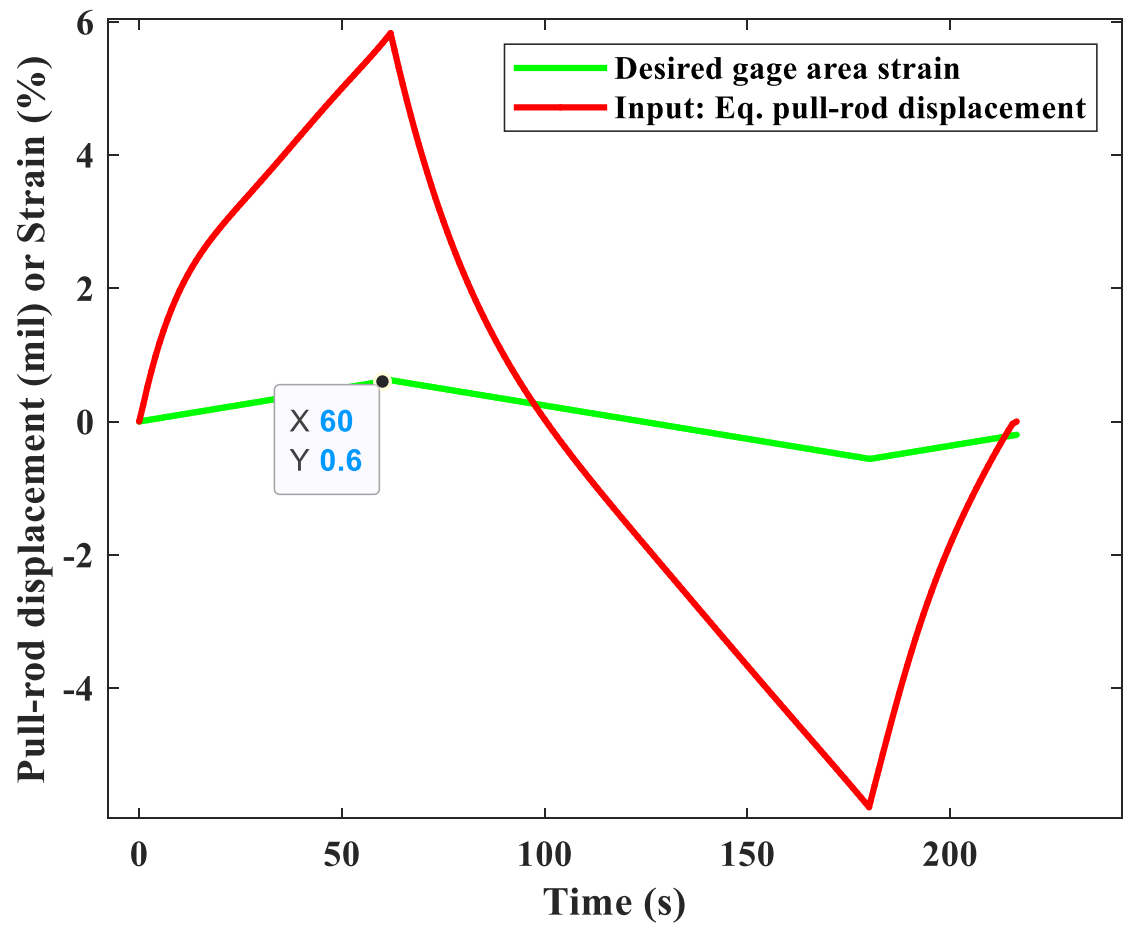

Figure 3. 11 Example first cycle input (out of different inputs applied to different cycles selected based on AI/ML based clustering technique) of pull-rod-displacement that was applied to ET-F58 (316SS base) specimen. 


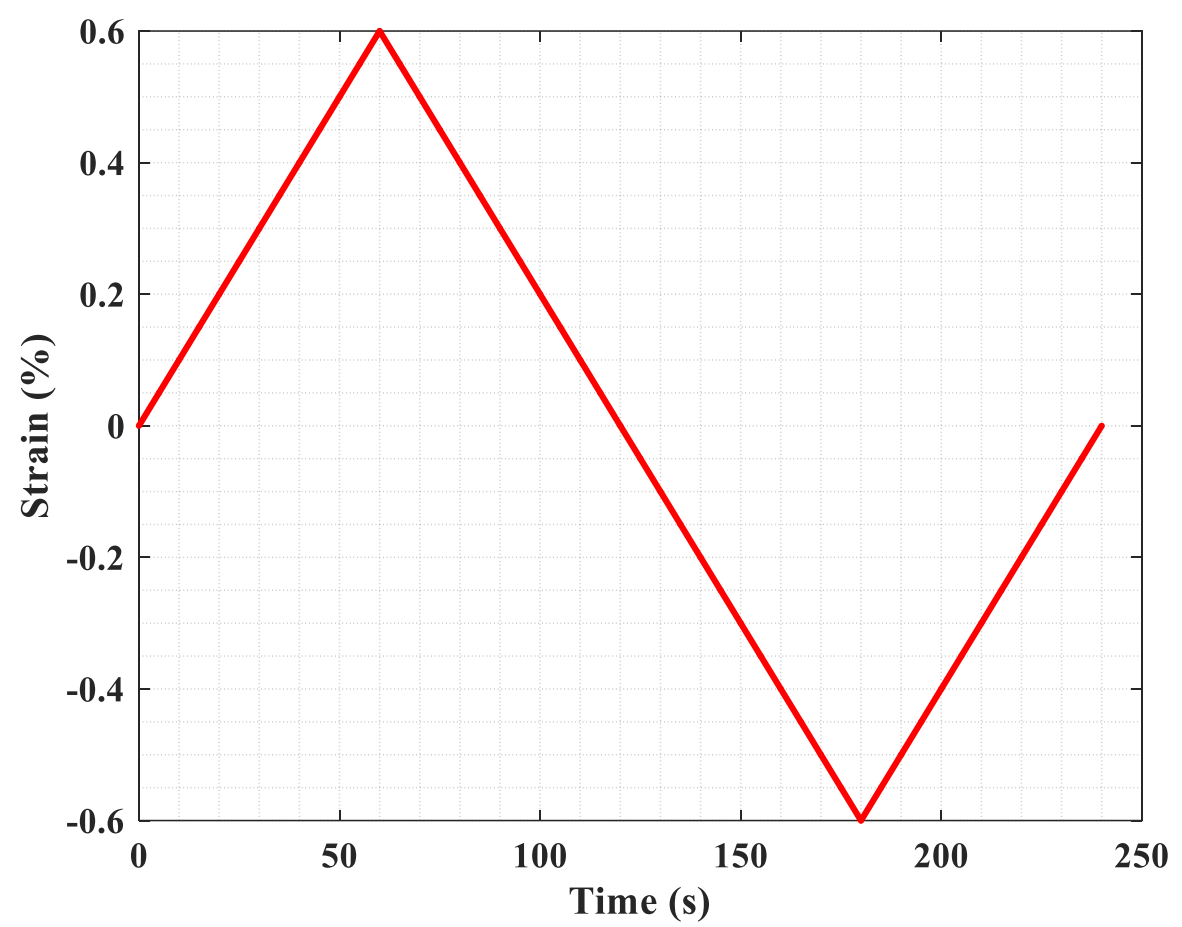

Figure 3. 12 A single-block of constant amplitude gage-area strain that was applied to ET-F59 (82/182 DMW butter weld) specimen until its failure.

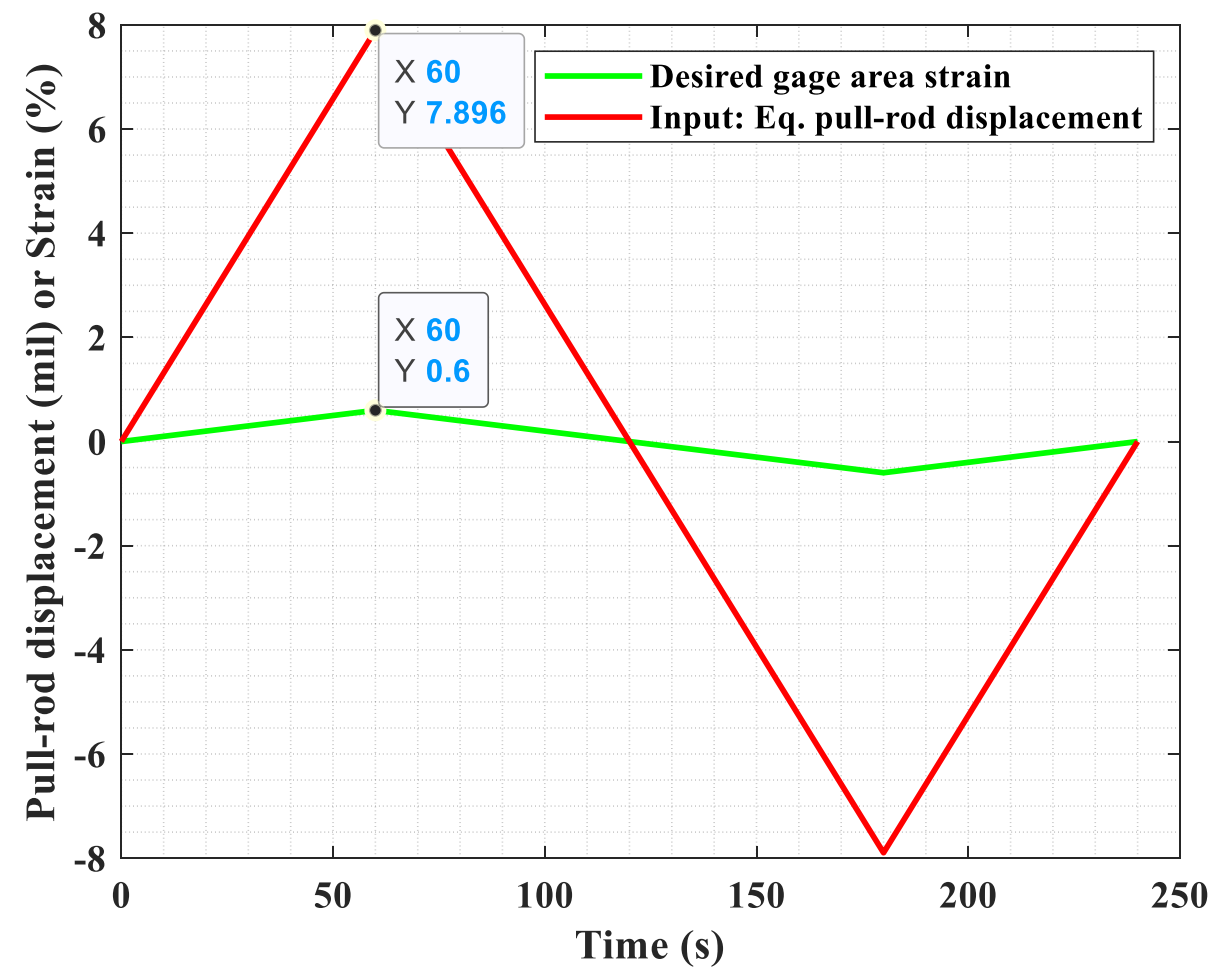

Figure 3. 13 Pull-rod-displacement (and corresponding intended strain amplitude) that was applied to ET-F60 (82/182 DMW filler weld) specimen until its failure. 


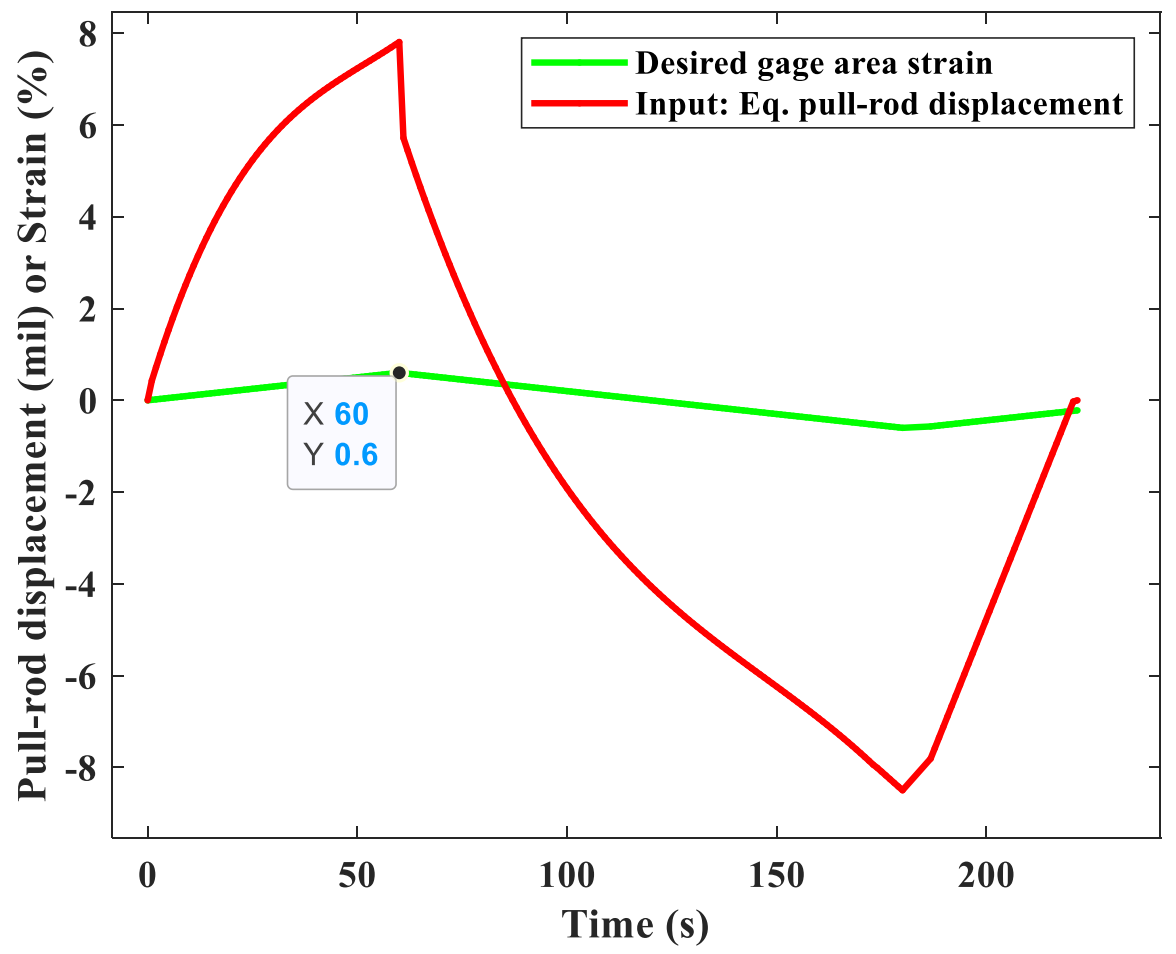

Figure 3. 14 Example first cycle input (out of different inputs applied to different cycles selected based on AI/ML based clustering technique) of pull-rod-displacement that was applied to ET-F61 \& EN-F62 (82/182 DMW filler weld) specimens.

\section{Al/ML Guided Seemingly Strain-Controlled Fatigue Test While Actually Not Controlling the Strain}

Low cycle fatigue evaluation technique primarily based on strain-controlled fatigue test data such as using strain versus life $(\varepsilon-N)$ curves. For generating the $\varepsilon-N$ curves, the fatigue test to be conducted using strain cycles with constant strain amplitude. This is required for generating $\varepsilon-\mathrm{N}$ curves under both in-air and PWR-water conditions. Although conducting strain-controlled fatigue tests under in-air condition is not an issue, since an extensometer can be used for measuring the strains and then those strain measurements can be used as feedback (to the test frame controller) to maintain the desired strain amplitude and strain rates. However, controlling strain in a PWR water test is a challenge, since an extensometer cannot be placed in a narrow autoclave (typically used in a high-temperature-pressure PWR-loop). This is due to lack of space inside an autoclave that houses the test specimen. In addition, installing a contact-type extensometer in the path of a high-pressure flow can be a challenge. These difficulty of using an extensometer in a PWR-water loop led us to use an outside-autoclave displacement sensor instead. This is by measuring the displacement of pull-rods (refer to Figure 3.5) and using those measurements to control the gauge area strain of a PWR-water specimens. To achieve a desired strain amplitude and strain rate the corresponding pull-rod displacement amplitudes and its rates (or equivalent time information) need to be fed to the test frame. In earlier ANL conducted fatigue tests the pull-rod displacements were chosen based on the strain versus pull-rod data obtained during a tensile test. Like in any contestant-amplitude strain-controlled fatigue tests, the input pull-rod-displacements are maintained same for the entire fatigue life. Although pull-rod-displacement-control method was used as a solution 
to conduct the desired train-controlled fatigue tests, but question is that can a pull-rod controlled tests generate the desired strain. In our study (based on in-air fatigue tests), we found that a pull-rodcontrolled based fatigue tests can lead to substantially different cyclic strain amplitudes and its rates compared to the desired constant cyclic strain amplitudes and its rates. These issues led us to think how to improve the pull-rod-displacement-control based tests such that a desired strain amplitude and rates can be achievable. For the purpose we used AI/ML based techniques such as Scikit-learn library [1] based k-mean clustering technique to improve our pull-rod-control based test method. The theoretical background of the k-mean clustering technique is briefly discussed in section 2 . The k-mean technique is an unsupervised AI/ML technique which segregate the data of inter-dependent field variables (can be considered as inter-dependent dimensions in a high-dimensional feature space) to a few groups or clusters. For example, in a fatigue tests the cycle-dependent sensor observations can be clustered into few groups based on which the test parameter can be selected. This is by assuming each time-series data stream as individual dimensions and they are dependent to each other. For example, the cyclic maximum and minimum amplitude of gauge strain amplitude (measured through an extensometer), pull-rod displacements (measured through a pull-rod displacement sensor), frame actuator displacement (measured through test frame LVDT sensor) and stress (measured through frame load cell) are assumed inter-dependent and their cyclic variation can be clustered into few groups. Based on this grouping the corresponding median fatigue cycle and the corresponding pull-rod-displacement versus strain data (in previously conducted pull-rod-displacement controlled in-air test) can be selected to generate the input test parameters for the individual clusters. This process can lead to a multi-step fatigue testing approach with varying pull-rod-displacement inputs rather than a fixed set of test inputs for the entire fatigue life. In the mentioned AI/ML guided test procedure, a set of test inputs were only repeated for the particular set of fatigue cycles, which were estimated from the above discussed k-mean clustering technique. Note that the test frame controller does not allow feeding thousands of different sets of parameters to achieve the strain amplitudes and rates during entire fatigue domain with thousands of fatigue cycles. Also coding different sets of parameters for each and every fatigue cycle is cumbersome and error prone. Hence, we believe the above-mentioned AI/ML based clustering technique is a possible solution to achieve seemingly strain controlled tests while actually not controlling the strains. To demonstrate the approach, we conducted different fatigue tests using both 316SS base metal and DMW filler/butter weld specimens (refer to Table 3.1 for detail test conditions) and the corresponding results are discussed below.

\subsection{SS Base-Metal Fatigue Test Results}

The AI/ML guided tests for 316SS base metal are conducted for ET-F58 in-air test. The test parameters are generated based on the cluster analysis of ET-F49 test data. This is by clustering the inter-dependent cycle versus maximum and minimum amplitude of gauge strain, pull-rod displacements, frame actuator displacement and stress data. Eight inter-dependent time-series data stream or dimensions were considered for development of the discussed k-mean clustering model. Figure 4.1 shows only the example 2-dimensional dependency between frame maximum actuator displacement and corresponding specimen gage area maximum strain. Whereas, Figure 4.2 shows the estimated cycle versus corresponding cluster numbers showing how the entire ET-F49 fatigue-test data clustered into only 5 different groups/clusters based on all the 8 dimensions. Based on this cluster analysis, the test parameters for the ET-F58 test case were chosen. Figures 4.3, 4.4 and 4.5 shows the ET-F58 test results 
and their comparison with respect to non-AI/ML-guided test conditions (such as for ET-F13 and ET-F49 fatigue tests). For example, Figure 4.3 shows the observed cycle versus strain amplitude in different fatigue tests conducted using the mentioned 316SS base metal specimens. Whereas, Figure 4.4 shows the corresponding observed cycle versus strain rate. Figure 4.5 shows the corresponding observed cycle versus max./min stress. From Figure 4.3, the ET-F49 observed strain amplitude substantially reduced to $0.45 \%$ although it was originally intended to achieve a strain amplitude of $0.65 \%$. This is almost a reduction of $31 \%$. Similarly, from Figure 4.4 the strain rate for ET-F49 test was reduced to $0.07 \%$ although it was originally intended to achieve a strain rate of $0.1 \% / \mathrm{s}$. This is approximate a strain rate drop of $30 \%$ from the intended strain rate. Contrary, in the AI/ML guided test case (ET-F58), it can be found that the cyclic strain amplitude varies approximately within a range of 0.6 to $0.65 \%$ for majority of the fatigue life, while the intended strain amplitude was $0.6 \%$. Similarly, from the Figure 4.4, the observed strain rates for ET-F58 specimen broadly follow the intended strain rate of $0.01 \% / \mathrm{s}$. Note that strain rate is one of the major parameters that affect the environmental correction factor $\left(\mathrm{F}_{\mathrm{en}}\right)$. Hence it is essential to control the desired strain rates in a PWR-water test case, otherwise the resulting $\mathrm{F}_{\text {en }}$ would be highly erroneous.

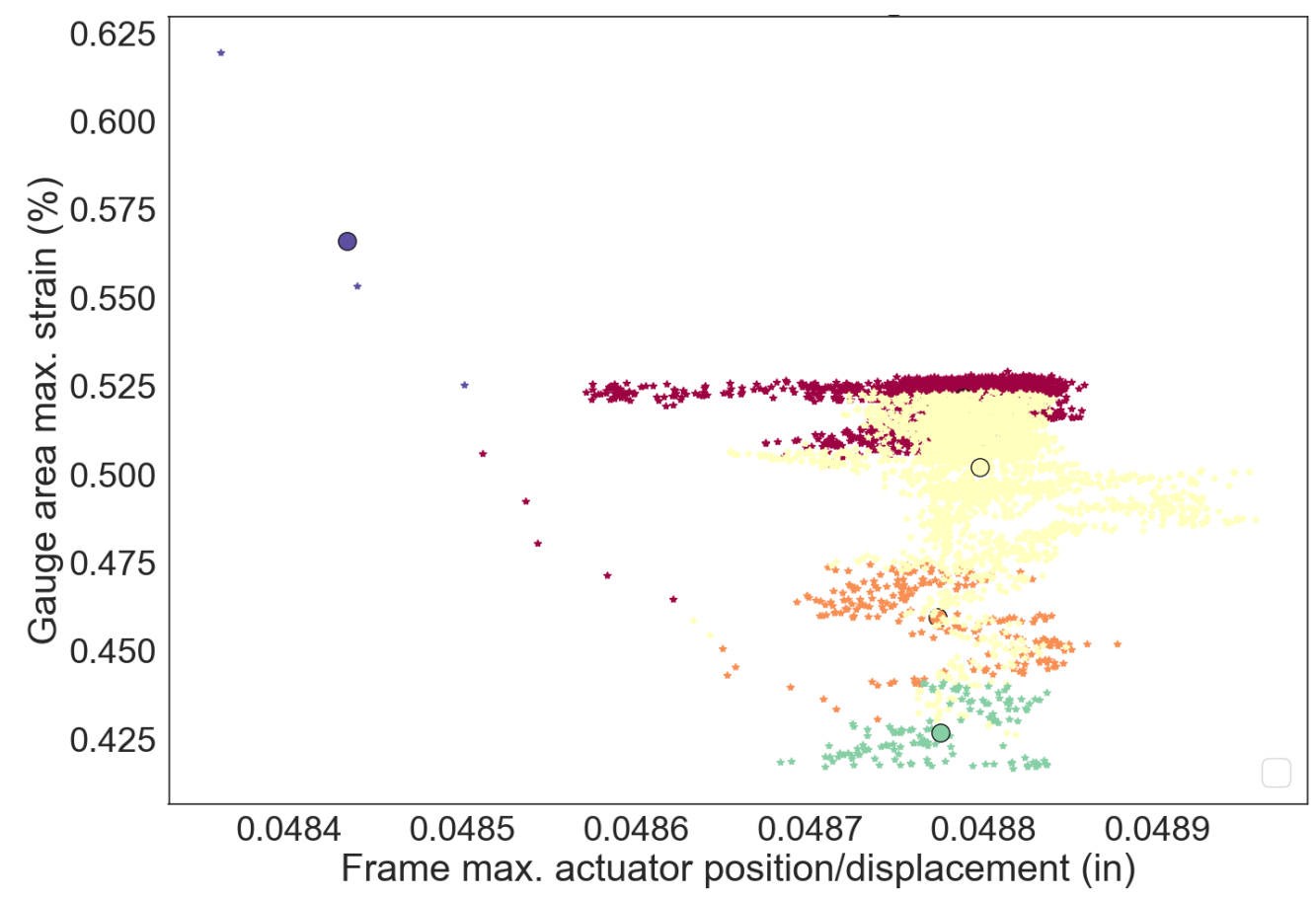

Figure 4. 1 A 316SS fatigue-test (ET-F49) based clustered data showing the example 2-dimensional dependency between frame maximum actuator displacement and corresponding specimen gage area maximum strain. Raw data in an individual cluster (plotted with single color) are reparented by dots whereas, the solid circles represent the corresponding cluster center. 


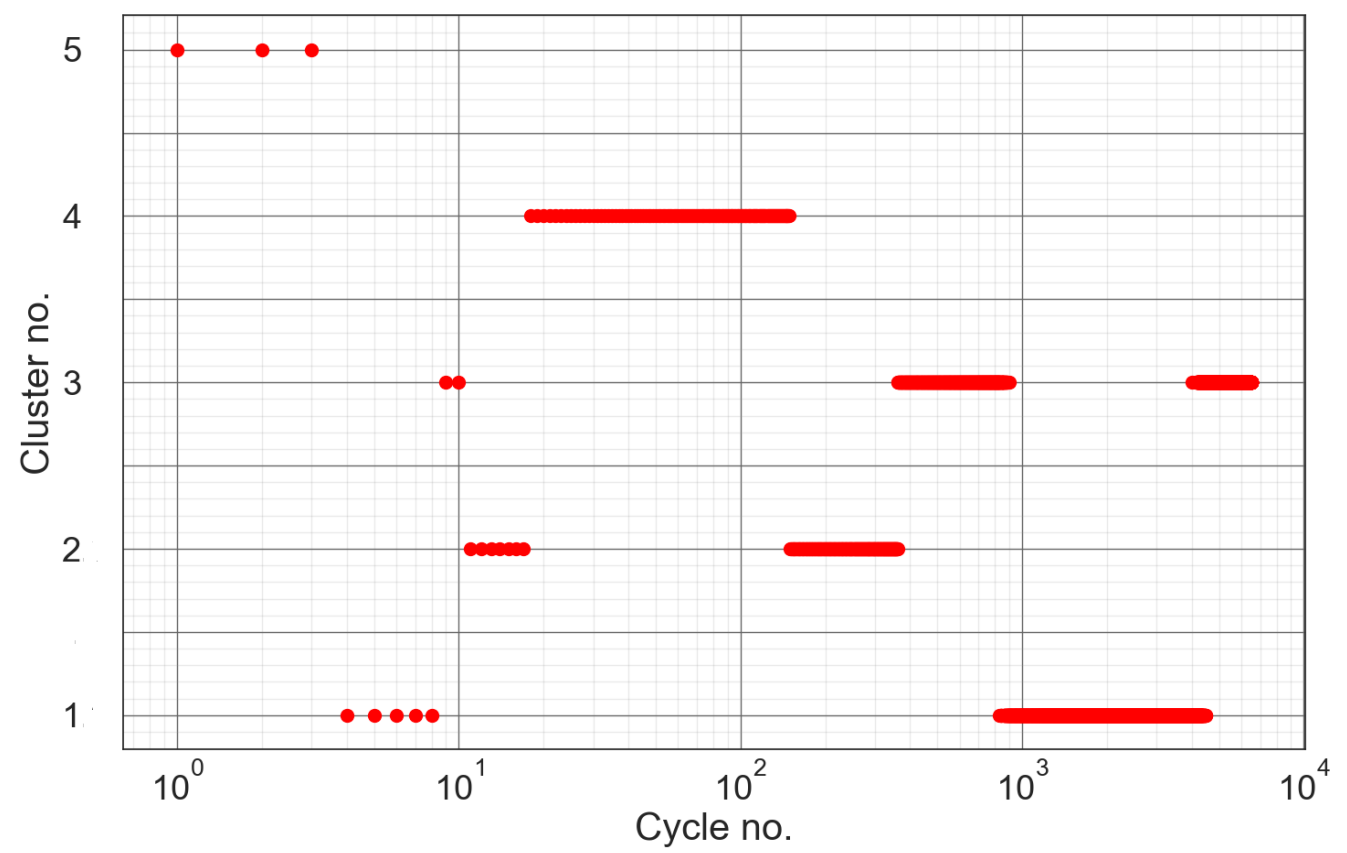

Figure 4. 2 Cycle versus corresponding cluster numbers showing how the entire ET-F49 fatigue-test data clustered into only 5 different groups/clusters based on which the ET-F58 test parameters were chosen.

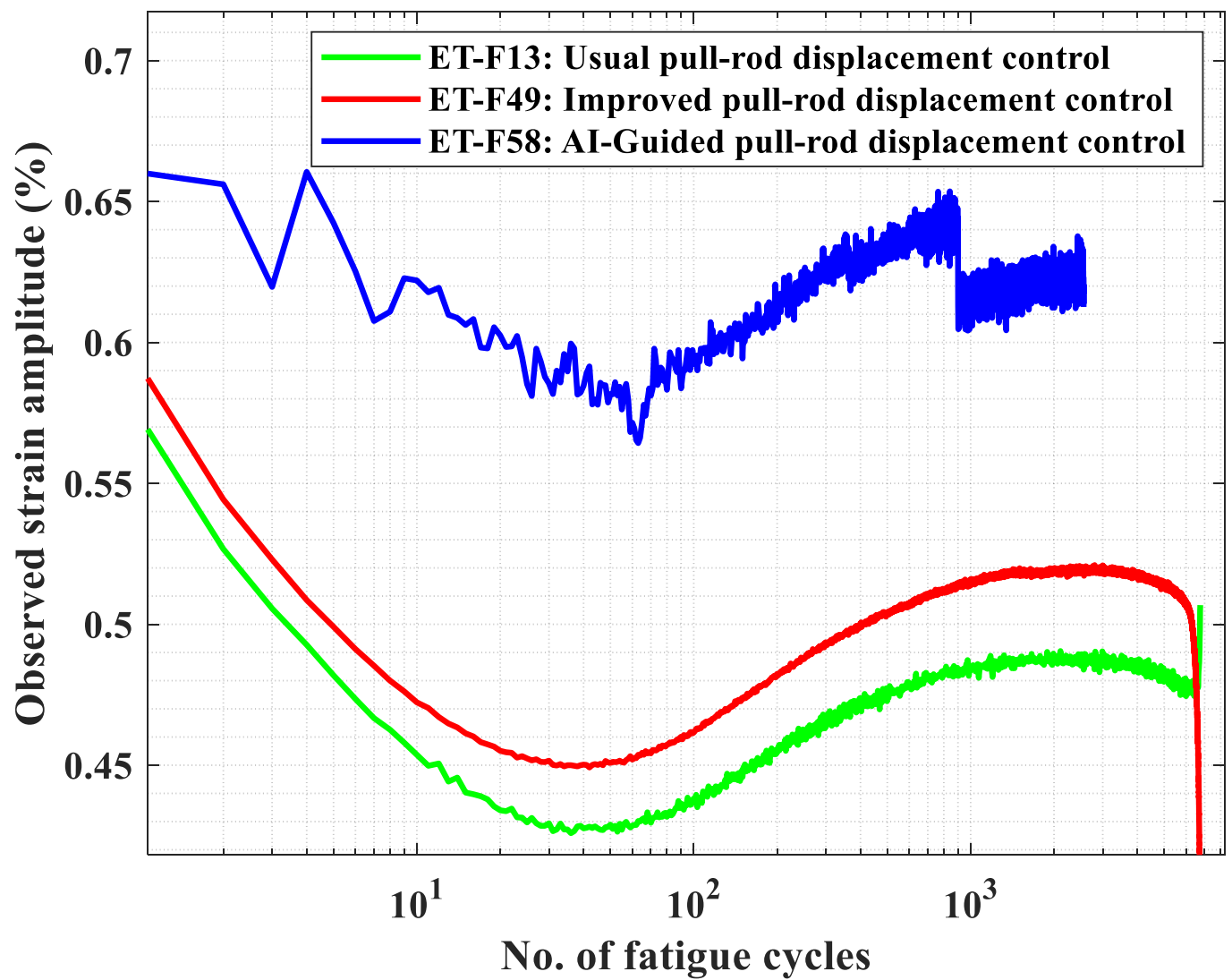

Figure 4. 3 Observed cycle versus strain amplitude in different fatigue tests conducted using 316SS base metal specimens. 


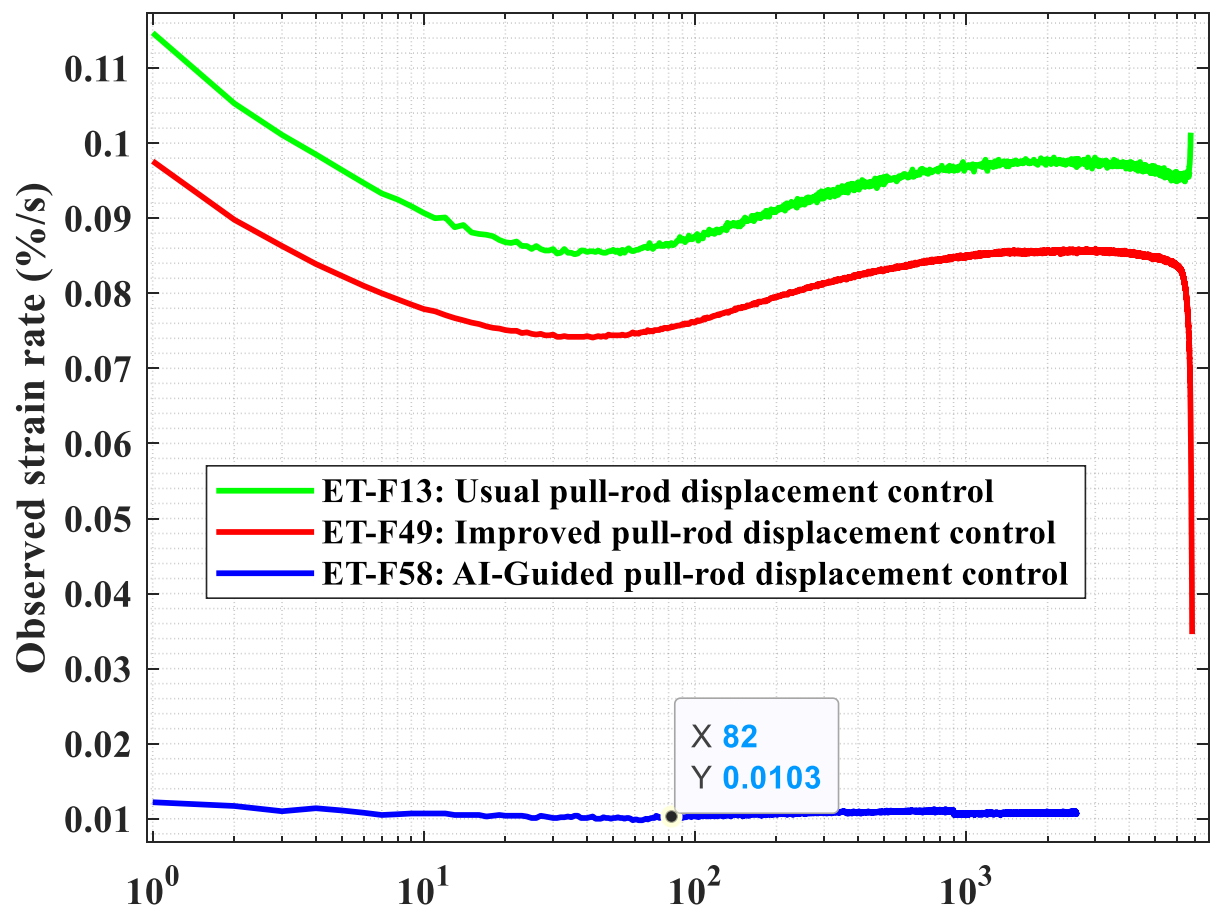

No. of fatigue cycles

Figure 4. 4 Observed cycle versus strain rate in different fatigue tests conducted using 316SS base metal specimens.

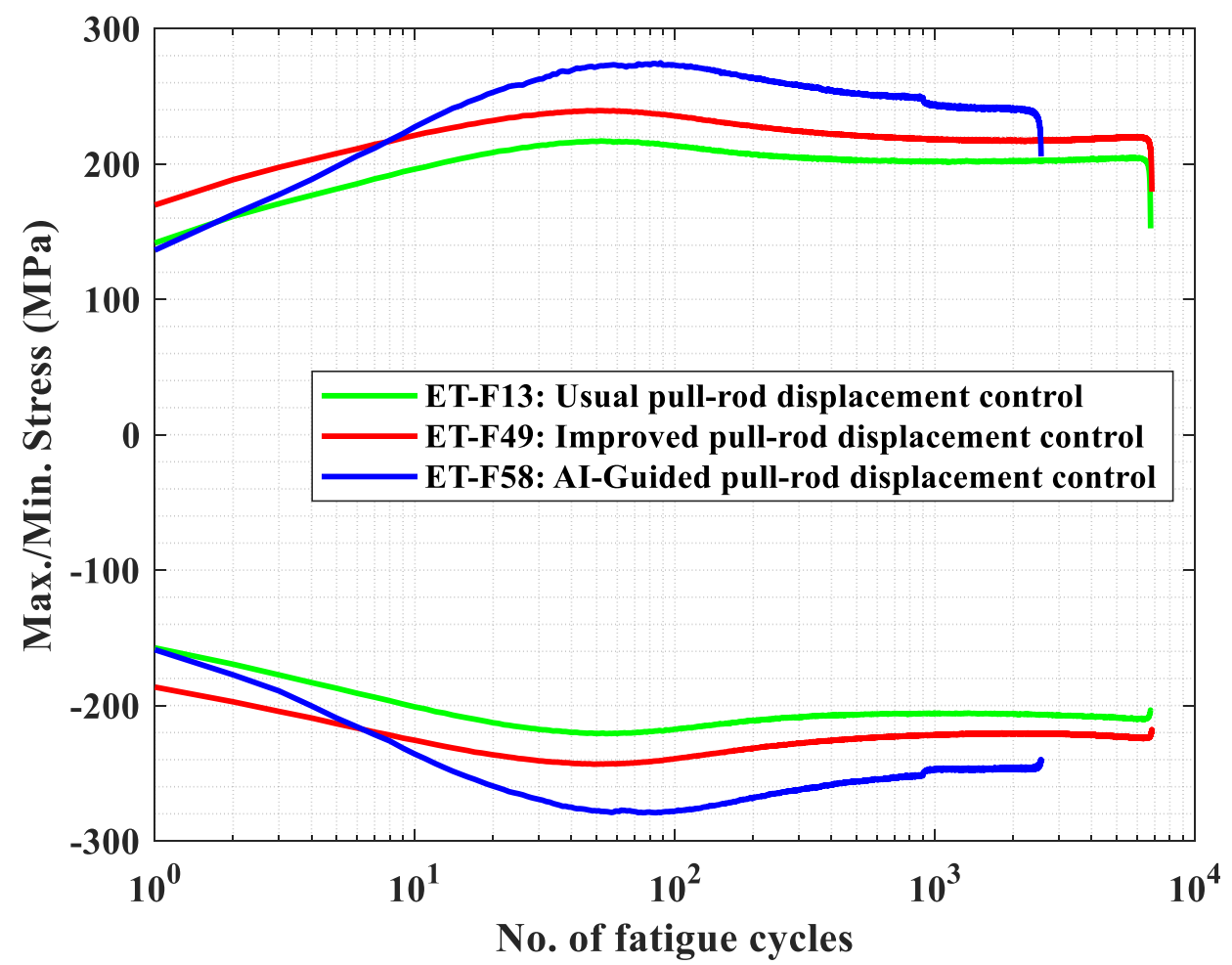

Figure 4. 5 Observed cycle versus max./min stress in different fatigue tests conducted using 316SS base metal specimens. 


\subsection{DMW Filler/Butter-Weld Fatigue Test Results}

The AI/ML guided test procedure was used for improving the fatigue test of DMW specimens. First a usual pull-rod-controlled based in-air test conducted to have the base data for cluster analysis and pullrod-displacement versus strain mapping data. For the purpose ET-F60 in-air test was conducted (refer to section 3 for test conditions). Using the resulting cycle versus maximum and minimum amplitude of gauge strain, pull-rod displacements, frame actuator displacement and stress data, the k-Mean clustering (refer to section 2.6) simulation was performed to group the entire fatigue domain to 9 clusters (in addition to first cycle). Figure 4.6 shows ET-F60 test case based clustered data showing the example 2dimensional dependency between frame maximum actuator displacement and corresponding specimen gage area maximum strain. Figure 4.7 shows the cycle versus corresponding cluster numbers showing how the entire ET-F60 fatigue-test data clustered into only 9 different groups/clusters. Based on this cluster analysis results, the median cycles of the clustered-cycles were selected to consider the corresponding cyclic stroke versus strain data (from ET-F60 test data) for deriving the intermittent mapping function between output pull-rod-displacement and the input strain. The mapping functions were modeled to predict the output pull-rod-displacement for a given input of strain amplitude and rates. Using this mapping function, the intermittent cycle pull-rod-displacements were estimated for the corresponding desired strain amplitude and rates. The estimated pull-rod-displacements were coded to the test frame controller as input or control parameters. Note that (for both the 316SS and DMW test cases) the first cycle data was not included in the cluster analysis. This is due to its specific tensile/monotonic behavior associated with its first quarter loading. First cycle was directly mapped (between the input strain and the output pull-rod-displacement) and resulting time versus pull-roddisplacement input were coded to test frame controller for first cycle loading only. Afterward, the intermittent time versus pull-rod-displacement input were repeated for the respective clustered cycles. Based on these AI/ML guided test-frame inputs two DMW metal specimens (ET-F61 under $300{ }^{\circ} \mathrm{C}$ inair conditions and EN-F62 under $300{ }^{\circ} \mathrm{C}$ PWR-water conditions) were fatigue tested. Example plot showing the time versus pull-rod-displacement input is shown in Figure 3.14. To check the efficacy of the AI/ML guided method (in respect to DMW weld specimens) the ET-F61 results are compared against the ET-F60 results. For example, Figure 4.8 shows the comparison of observed cycle versus strain amplitudes. Whereas as Figure 4.9 shows the corresponding comparison of observed cycle versus strain rates. Figure 4.10 shows the corresponding observed cycle versus max./min stress in addition to the max./min stress observed in EN-F62 water-loop tests for which strain was not measurable. From the Figure 4.8 for the ET-F60 test case the strain amplitude varies substantially from the intended amplitude of $0.6 \%$. The lowest achieved amplitude is approximately of $0.42 \%$. This leads to an error of $30 \%$. Whereas for AI/ML guided test ET-F61, the strain amplitude falls in a range of $0.6-0.65 \%$ during the majority of test specimen fatigue life. From the Figure 4.9, the observed strain rate for ET-F60 specimen varies monotonically over the fatigue life. It reduces to an approximate value of $0.007 \% / \mathrm{s}$ whereas the intended strain rate for the test was $0.01 \% / \mathrm{s}$. This leads an approximate error of $30 \%$. Whereas from the corresponding AI/ML guided in-air test (ET-F61), the observed strain rate broadly varies about desired strain rate of $0.01 \% / \mathrm{s}$, during most of the fatigue life. From Figures 4.8 and 4.9 it can be seen that during final failure, the specimen experiences asymptotic increase of strain amplitude and strain rate. This is due to the final-failure regime related instability which lead to tensile rupture rather than the intended fatigue damage. Hence, last few cycle data in context of strain amplitude and strain rate control are not relevant. Figures 4.8 to 4.10 show also the comparison of results for corresponding strain- 
controlled test case (ET-F59), which was fatigue tested with strain amplitude and rate of $0.6 \%$ and $0.01 \% / \mathrm{s}$, respectively.

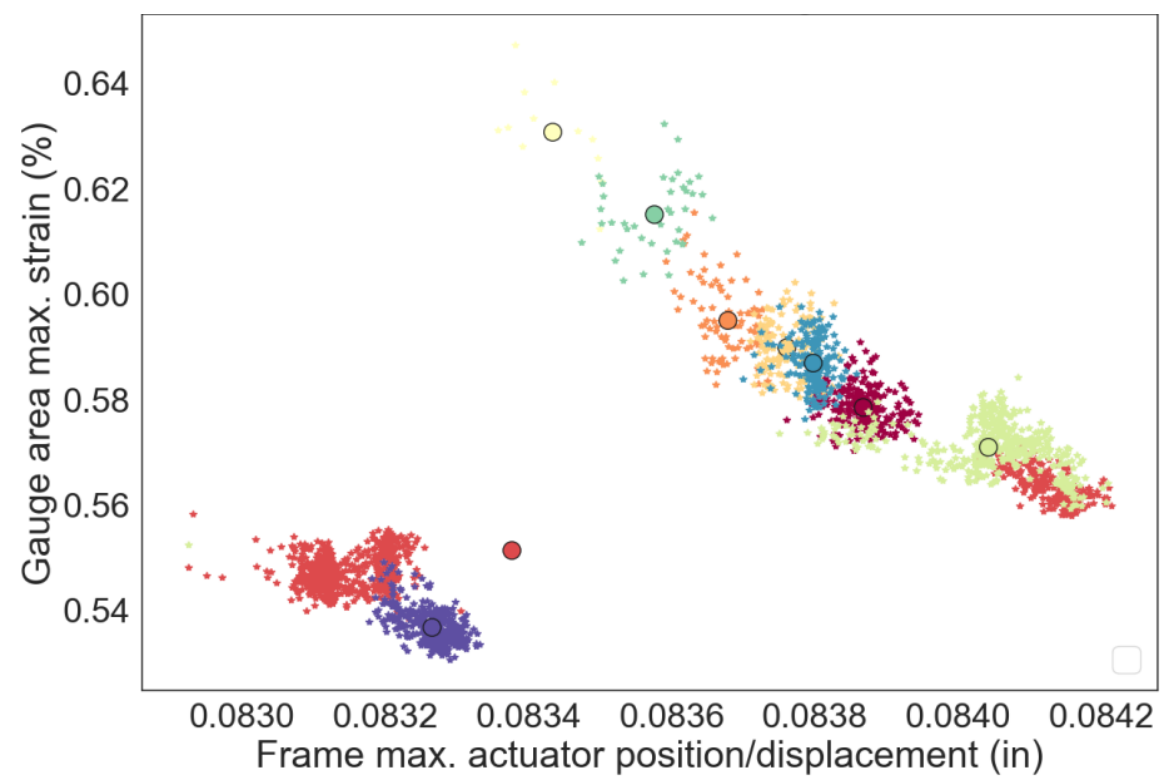

Figure 4. 6 A DMW fatigue-test (ET-F60) based clustered data showing the example 2-dimensional dependency between frame maximum actuator displacement and corresponding specimen gage area maximum strain. Raw data in an individual cluster (plotted with single color) are reparented by dots whereas, the solid circles represent the corresponding cluster center.

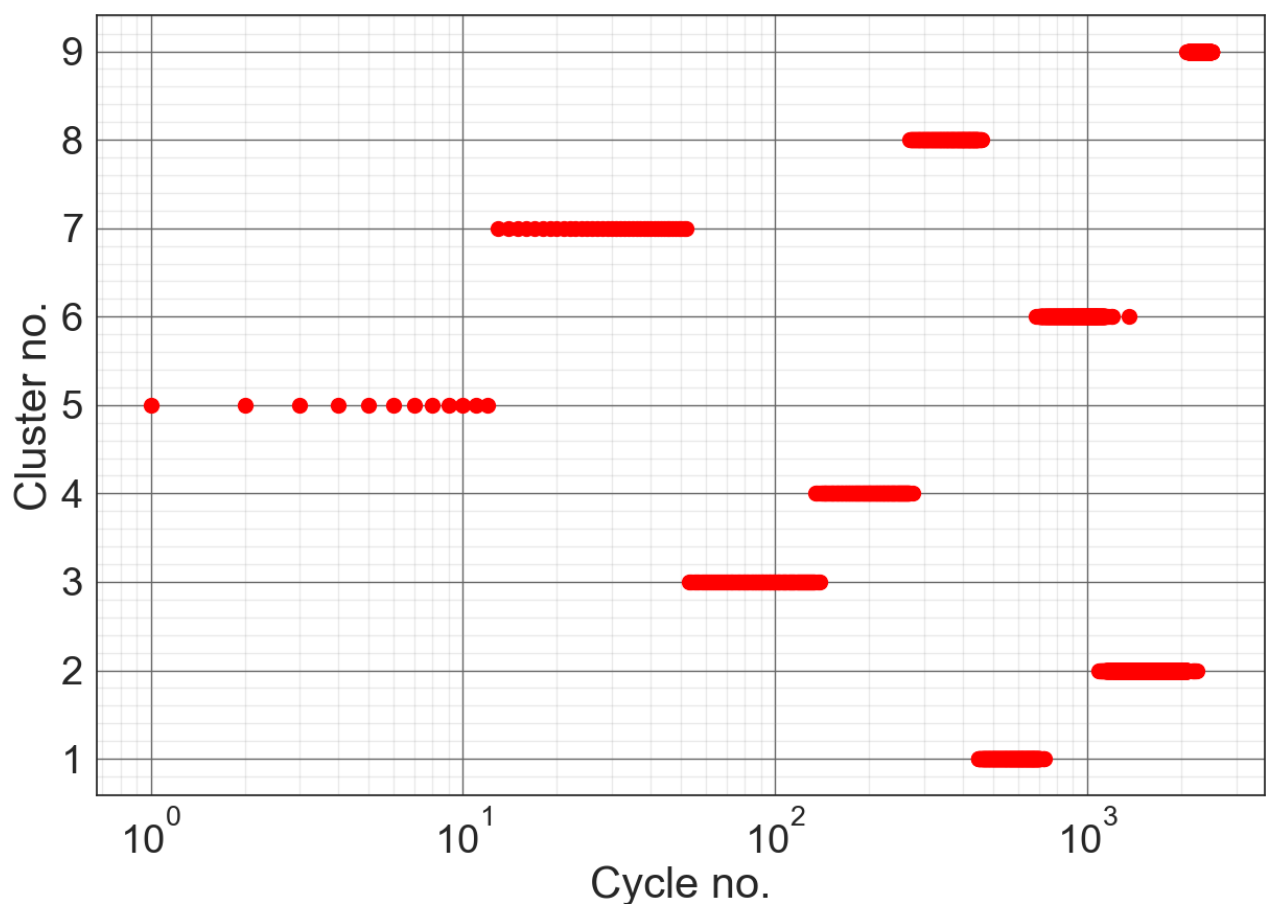

Figure 4. 7 Cycle versus corresponding cluster numbers showing how the entire ET-F60 fatigue-test data clustered into only 9 different groups/clusters based on which the ET-F61 and EN-F62 test parameters were chosen. 


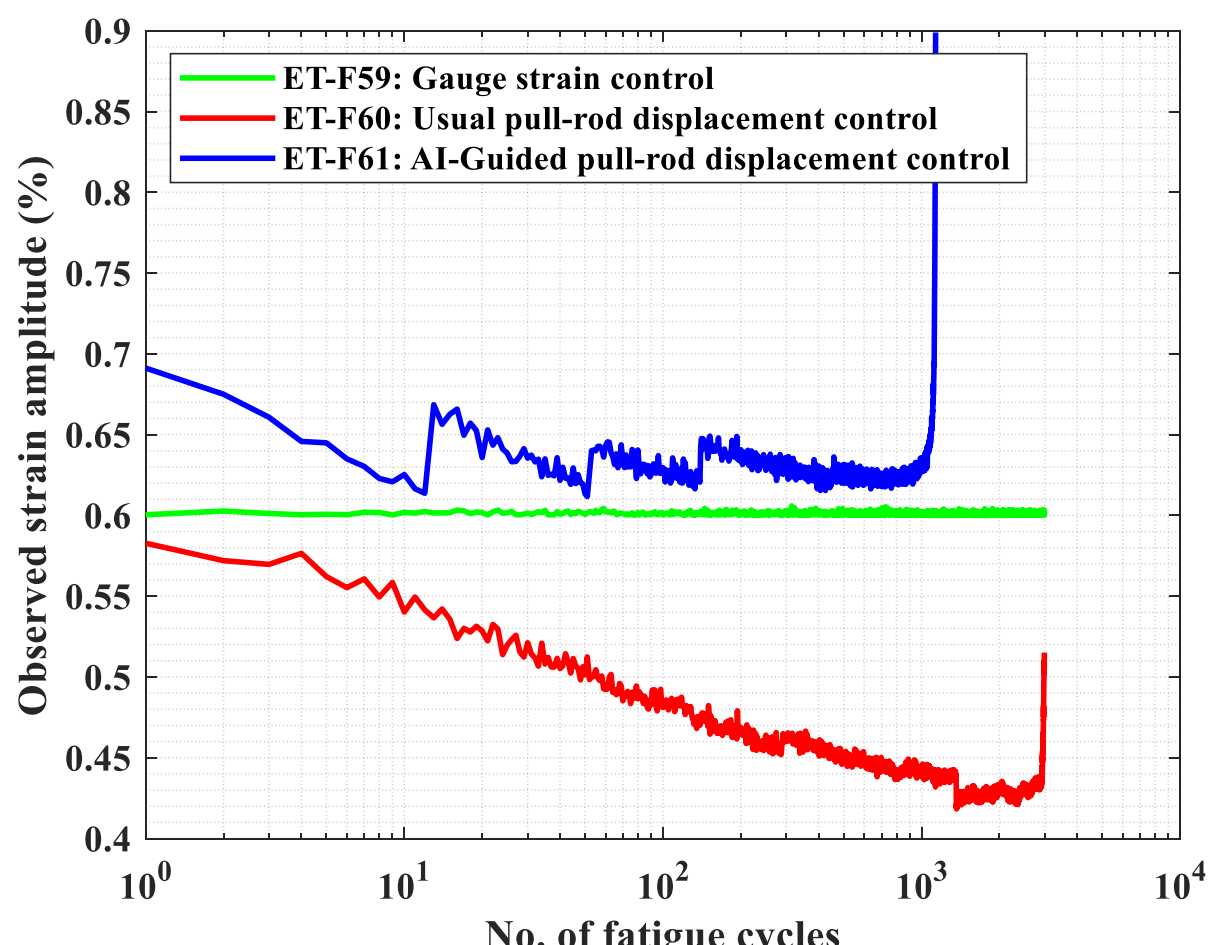

Figure 4. 8 Observed cycle versus strain amplitude in different fatigue tests conducted using DMW filler/butter metal specimens.

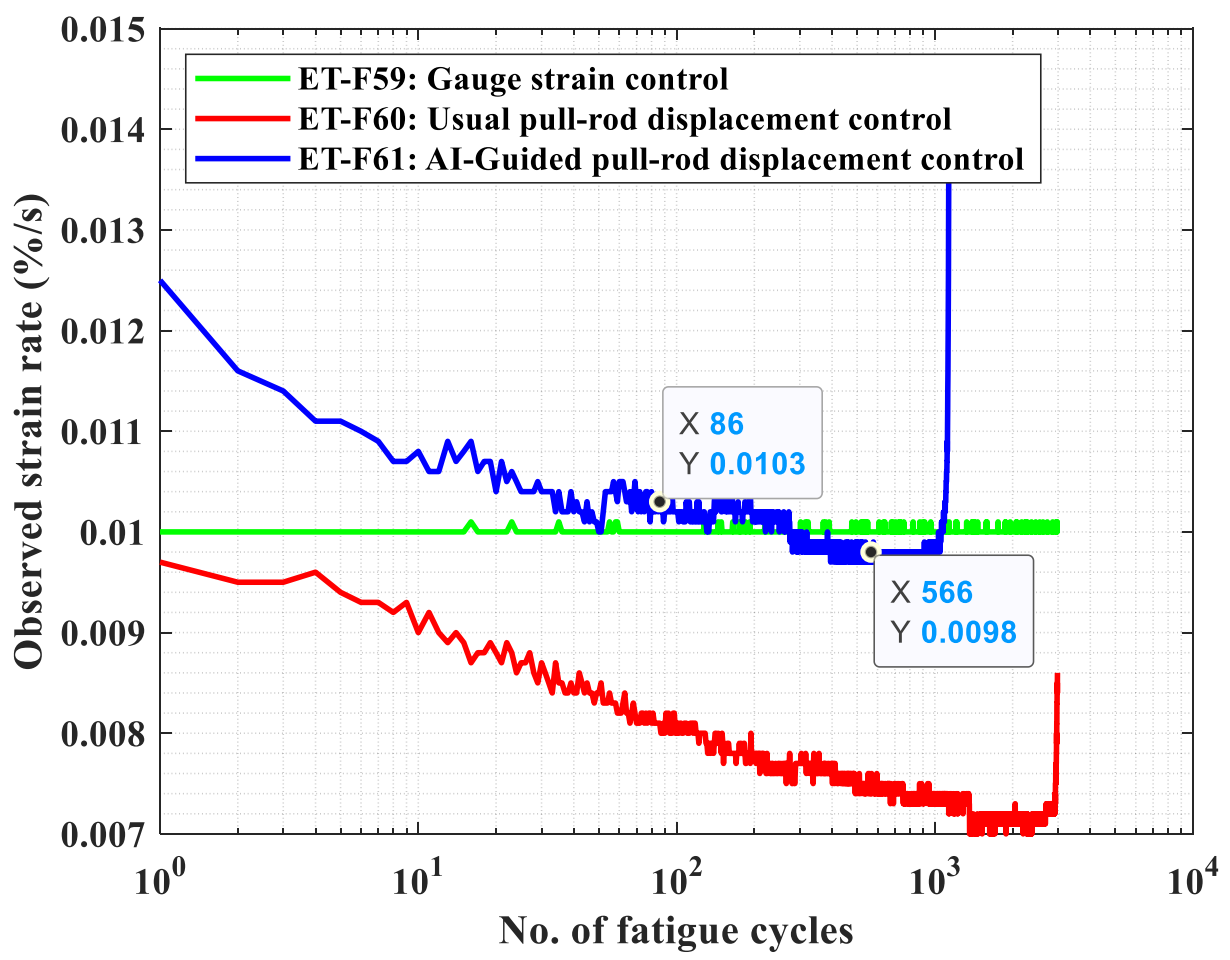

Figure 4. 9 Observed cycle versus strain rate in different fatigue tests conducted using DMW filler/butter metal specimens. 


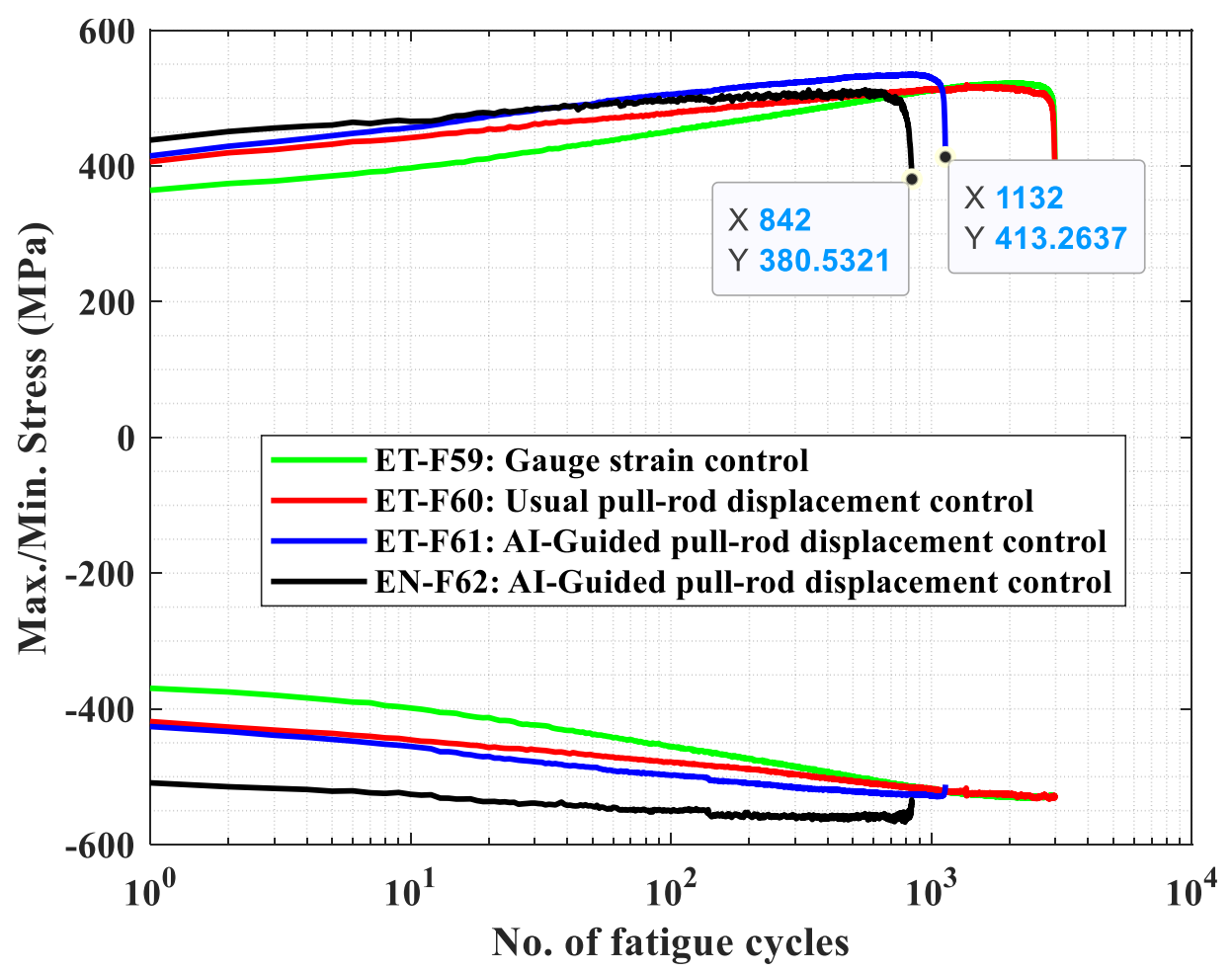

Figure 4. 10 Observed cycle versus max./min stress in different fatigue tests conducted using DMW filler/butter metal specimens.

\subsection{Observed Environmental Correction Factor for DMW Metal}

Environmental correction factor $\left(F_{e n}\right)$ needed to be estimated for DMW specimen. This is for using $S \sim N$ curve-based life estimation approach such as using the approach described in NUREG-6909 [20]. For estimating the $F_{\text {en }}$, two parallel tests were conducted under in-air and PWR-water environment but under similar loading. Considering an in-air life $\left(N_{\text {air }}\right)$ of 1132 cycles (obtained through in-air ETF61 test) and a PWR-water life $\left(N_{\text {water }}\right)$ of 842 cycles (obtained through PWR-water EN-F62 test), the environmental correction factor $\left(F_{e n}\right)$ for DMW (with strain rate of $0.01 \% / \mathrm{s}$ ) can be found as:

$$
F_{\text {en }}=\frac{N_{\text {air }}}{N_{\text {water }}}=\frac{1132}{842}=1.3444
$$




\section{Use of Al-Based Machine Learning and Deep Learning Techniques for Time-Series Strain Prediction from Other Sensor Measurements}

In this section, we discuss artificial intelligence (AI) based machine leaning (ML) and/or deep learning techniques (DL) approaches to predict time-series cyclic strain in an inaccessible autoclave. In an PWR-water test loop (refer to Figure 3.6), a narrow autoclave is being used to house the test specimen. The narrow autoclave along with high-pressure-high-temperature flow of coolant water don't allow to place an extensometer inside the autoclave. This makes difficulty to know how much strain being created in the gauge area of specimen. Note that, ASME code requires strain-controlled tests for low cycle fatigue assessment of reactor components. However, without measuring how much strain imposed on a specimen, it is difficult to ascertain the accuracy of the fatigue tests particularly conducted using the above mentioned PWR-water test loop. With the difficulty of measuring strain, we use the pull-rod-displacement measurements (refer to Figures 3.5 and 3.6) to control the fatigue test. However, controlling pull-rod displacement creates cyclic strain hardening/ softening leading to substantial cyclic variation of strains from the original anticipated strain (estimated based on tensile test-based pull-roddisplacement versus strain mapping function). Cyclic strain not only follows a complex hysteresis behavior with respect to applied pull-rod-displacement, but also the overall hysteresis behavior changes over time/cycles due to cyclic strain hardening/softening. Without the proper knowledge of generated strain in a PWR-water test specimen, it not only creates the difficult to ascertain the accuracy of the fatigue tests but also create issue of developing cyclic-plasticity material models which are based on the observed cyclic strain versus stress data. In the reported work, we attempted to solve the issue of predicting strain in an autoclave environment. This is by using AI based ML and/or DL, techniques such as exploring the use of Scikit-learn [1] and Keras [3] libraries. In the discussed results, we used both Scikit-learn based multi-variate regression (refer to section 2.3) and Keras based deep learning regression (refer to section 2.4) for time-series cyclic strain prediction. The cyclic strain predictions were made considering 4 independent dimensions such as time, pull-rod-displacement measurements, frame-actuator-displacement measurements and frame-load-cell measurements. This is a type of sensor fusion approach, in which multi-dimensional sensor measurements (from different type of sensors) combined/fused together to map some features. We also used the Scikit-learn based clustering techniques (refer to section 2.6) to cluster/group the entire fatigue life to multiple subdomains. This is to piecewise strain prediction for dealing with non-stationarity issues associated with cyclic hardening/softening under cyclic loading. First the approach was validated against in-air test data for which not only the input dimension data (time, pull-rod-displacement measurements, frame-actuatordisplacement measurements and frame-load-cell measurements) are available but also the output strain measurements are available (due to the possibility of using an extensometer to measure the gauge area strain). Two cases of validation were made: First using ET-F54 in-air test data, for which the DMW specimen was tested under unsymmetrical design-base fatigue loading (refer to section 3), second using ET-F61 in-air-test data for which the DMW specimen was tested under symmetrical fatigue loading (refer to section 3). Based on the validation results, we found Keras deep learning model (with ReLU activation function) produces best results and later used for predicting the strain under PWR-water loop test case such as of EN-F62 test case. The details of the results are presented below. 


\subsection{Validation case-1: Cyclic Strain Prediction for Fatigue Test Specimen Subjected to ET-F54 Unsymmetrical Design-Basis Cyclic Loading}

The AI/ML/DL learning models were tested from different aspects such as checking the effect of input dimensions for example can a single dimension (or input measurements) can be able to predict the strain accurately. Also, we checked which AI/ML/DL model and activation functions give best results and what are the computational time for each approach. We also tested the efficacy of the piecewise modeling approach using k-mean based clustering technique. The related test results with respect to ETF54 loading case were discussed below. Note that the ET-F54 specimen was a DMW filler weld specimen and was tested under in-air environment while subjected to unsymmetrical-design-basis cyclic loading. The pull-rod-displacement based loading parameters were selected based on the finite element model of PWR surge-line nozzle (refer to section 3).

\subsubsection{Study the effect of independent variables on accuracy of strain estimation under unsymmetrical design-basis cyclic loading}

Part of this study we checked the effect of individual input dimension. For the purpose we used Keras deep learning model (refer to section 2.4) with ReLU activation function. We only considered first 50 cycles for the mentioned input-dimension-dependency validation check. Note that we found Keras model with ReLU activation functions gives the best results which discussion follows. However, when a single dimension is used as input, then results are highly inaccurate as can be seen from Figures 5.1 to 5.4. This led us to use multidimensional inputs such as time, pull-rod-displacement measurements, frame-actuator-displacement measurements and frame-load-cell measurements. In all the further discussed results in the subsection 5.1 we used the above mentioned 4 input dimensions for the equivalent strain prediction. 


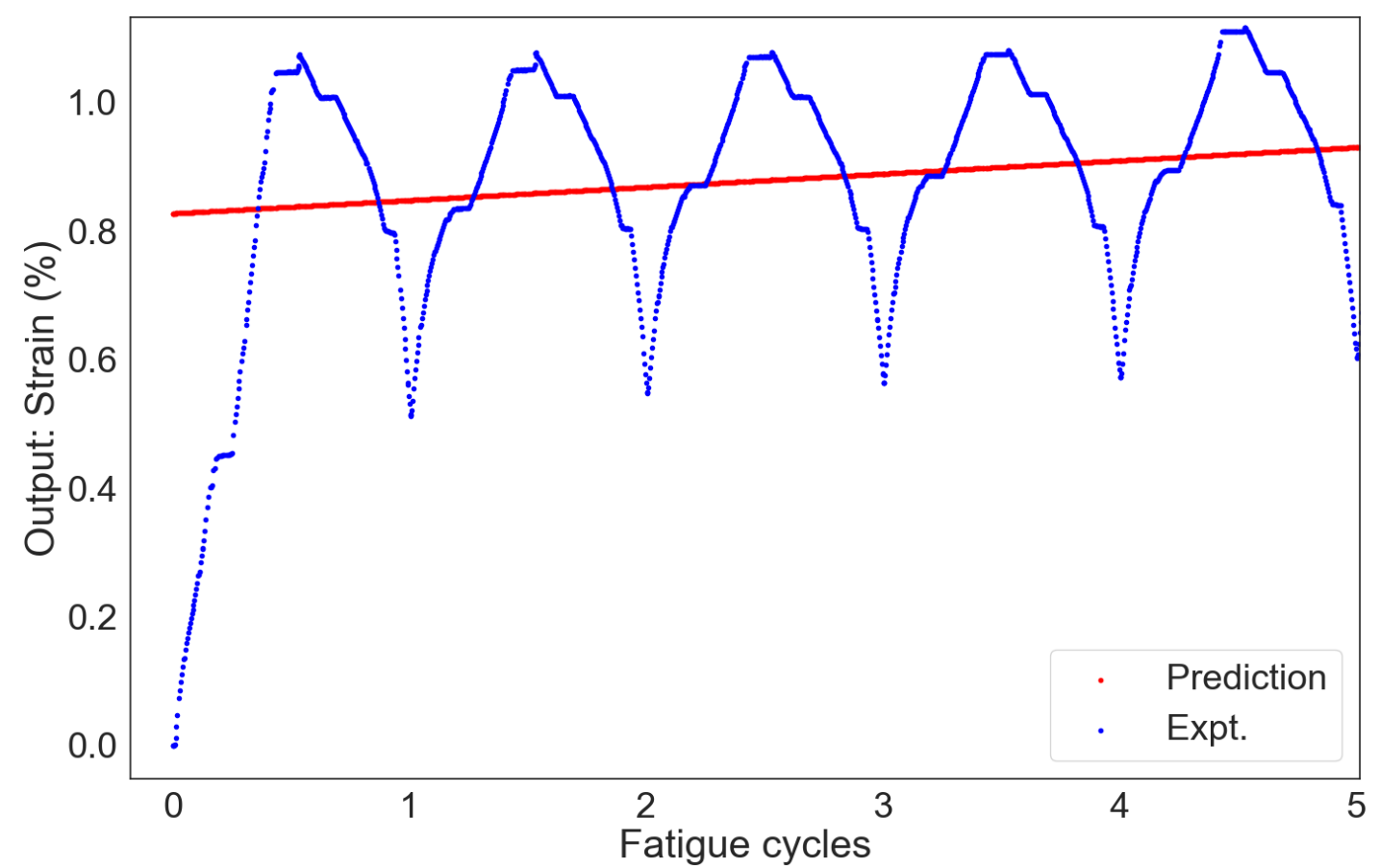

Figure 5. 1 Cyclic strain estimation using the time as the only independent variable.

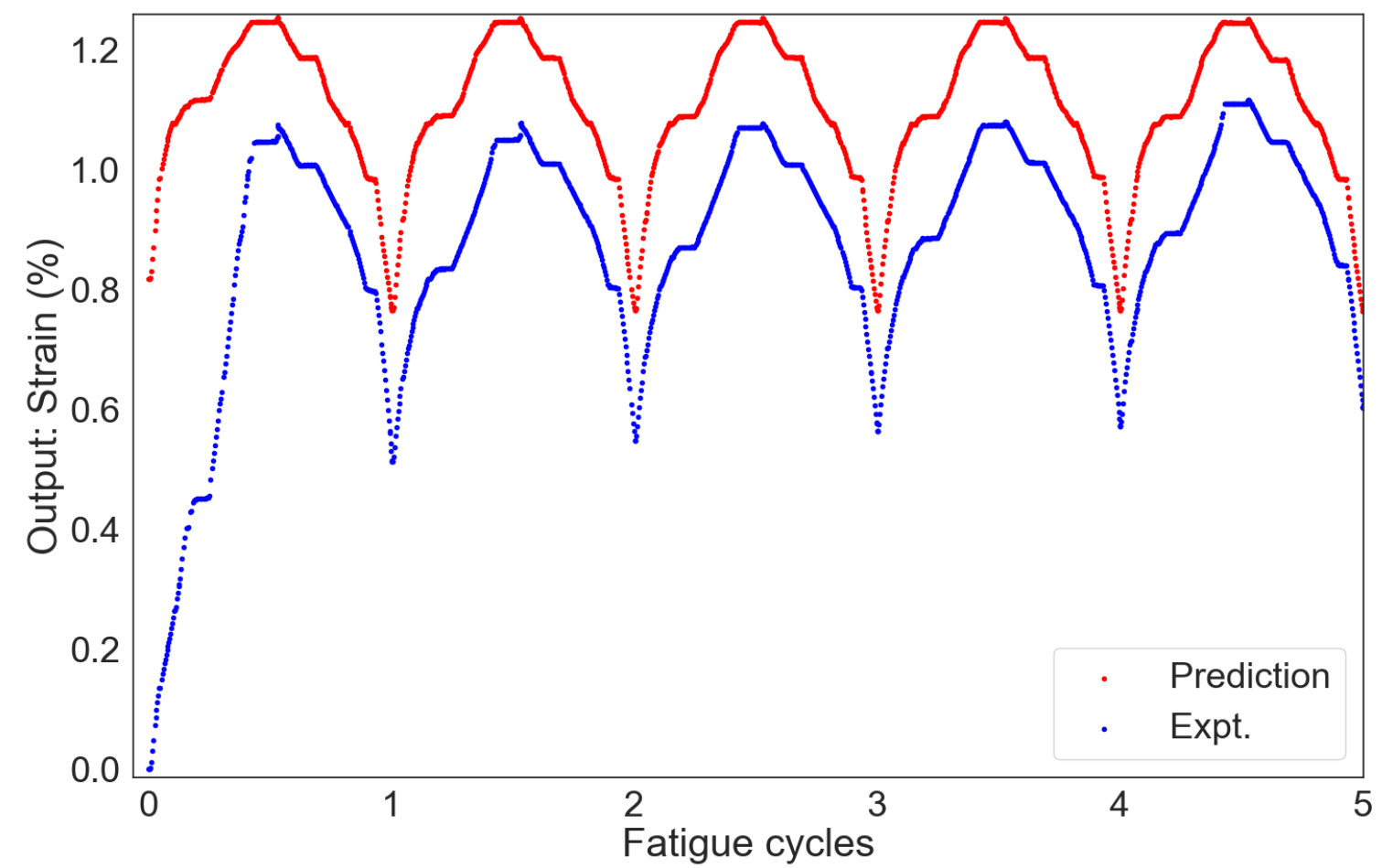

Figure 5. 2 Cyclic strain estimation using displacement of the test-frame actuator as the only independent variable. 


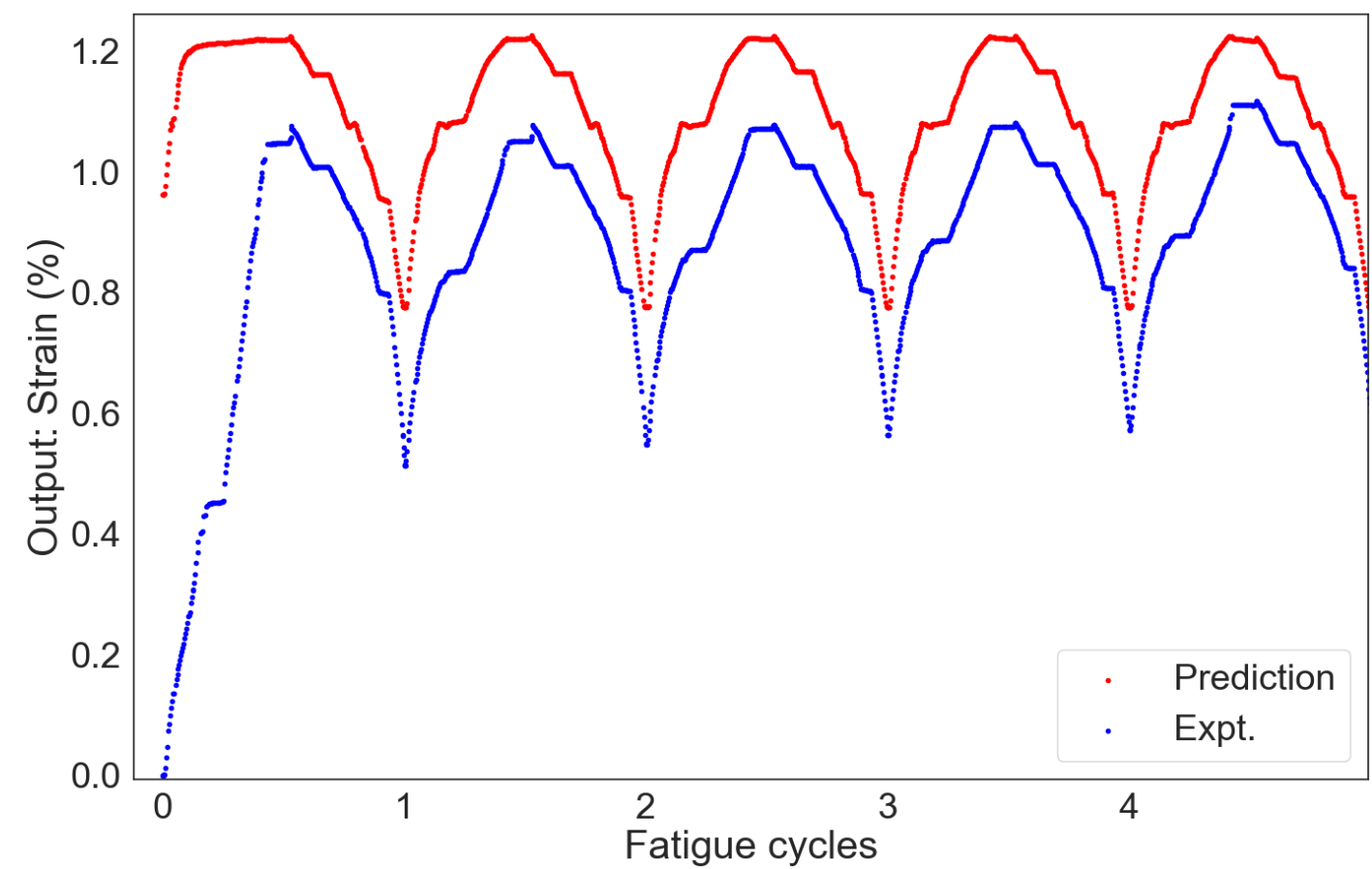

Figure 5. 3 Cyclic strain estimation using the load-cell measurement of the test-frame as the only independent variable.

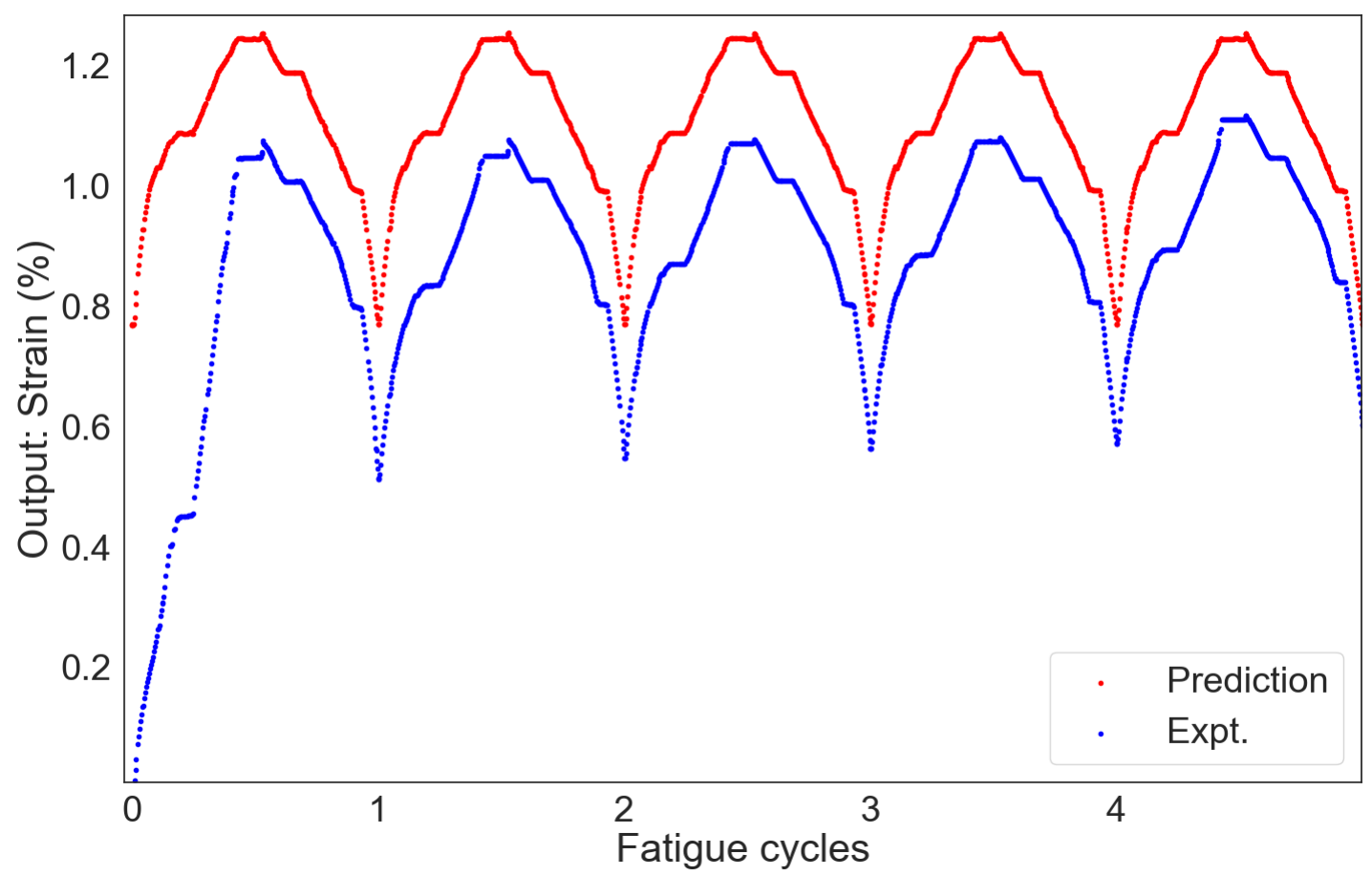

Figure 5. 4 Cyclic strain estimation using the pull-rod displacement as the only independent variable. 
5.1.2 Accuracy associated with different types of Al technique under unsymmetrical design-basis cyclic loading

As part of this study we checked the accuracy of different AI/ML/DL approach for prediction of cyclic strain. We considered first 50 cycles data for checking the model accuracy. The pool of 50 cycle data was randomly divided in to training and test data with 70\%/30\% ratio, which is a usual practice while testing $\mathrm{AI} / \mathrm{ML} / \mathrm{DL}$ algorithms. We conducted 100 randomized iterations (for randomly selecting the training and test data with $70 \% / 30 \%$ ratio. We checked the efficacy of Scikit-learn based multidimensional-linear regression technique and Keras based multi-dimensional DL regression techniques. We checked the efficacy of both Sigmoid and ReLU activation function for Keras DL models. For all the model cases (used for present AI technique accuracy checking), a single-step modeling approach was followed. Figures 5.5 to 5.8 show the results from Scikit-learn based model. Whereas Figures 5.9 to 5.12 and Figures 5.13 to 5.16 show the results from Keras model with Sigmoid and ReLU activation functions, respectively. Table 5.1 shows the summary of the overall finding that Keras DL model with ReLU activation function gives best results compared to other two options. However, from the Table 5.1 it can be found that Keras model consume more computational time compared to Scikit-learn based model. However, it may be worth to use Kreas model since the accurate of strain prediction would help to predict the accurate nonlinear shape of the strain versus stress hysterics curves, which will be later used for material models.

Table 5. $1 \mathrm{CPU}$ time and accuracy for 100 randomized iterations while predicting strain for first 50 cycles of ET-F54 test case.

\begin{tabular}{|c|c|c|c|}
\hline $\begin{array}{c}\text { AI/ML/DL } \\
\text { Techniques }\end{array}$ & $\begin{array}{c}\text { Scikit-learn AI/ML } \\
\text { Library with multi- } \\
\text { dimensional-linear } \\
\text { and single-step } \\
\text { regression }\end{array}$ & $\begin{array}{c}\text { Keras AI/DL library } \\
\text { with Sigmoid } \\
\text { activation function } \\
\text { and multi- } \\
\text { dimensional -single- } \\
\text { step regression }\end{array}$ & $\begin{array}{c}\text { Keras AI/DL library } \\
\text { with ReLU activation } \\
\text { function and multi- } \\
\text { dimensional -single- } \\
\text { step regression }\end{array}$ \\
\hline CPU Time $(s)$ & 49.2 & 7609.7 & 7702.0 \\
\hline $\begin{array}{c}\text { Accuracy: Highest } \\
\text { MSE observed }\end{array}$ & 0.00086 & 0.0008 & 0.00026 \\
\hline
\end{tabular}


A Hybrid Al/ML and Computational Mechanics Based Approach for Time-Series State and Fatigue Life Estimation of Nuclear Reactor Components

5.1.2.1 Cyclic strain estimation using AI/DL based Scikit-learn library

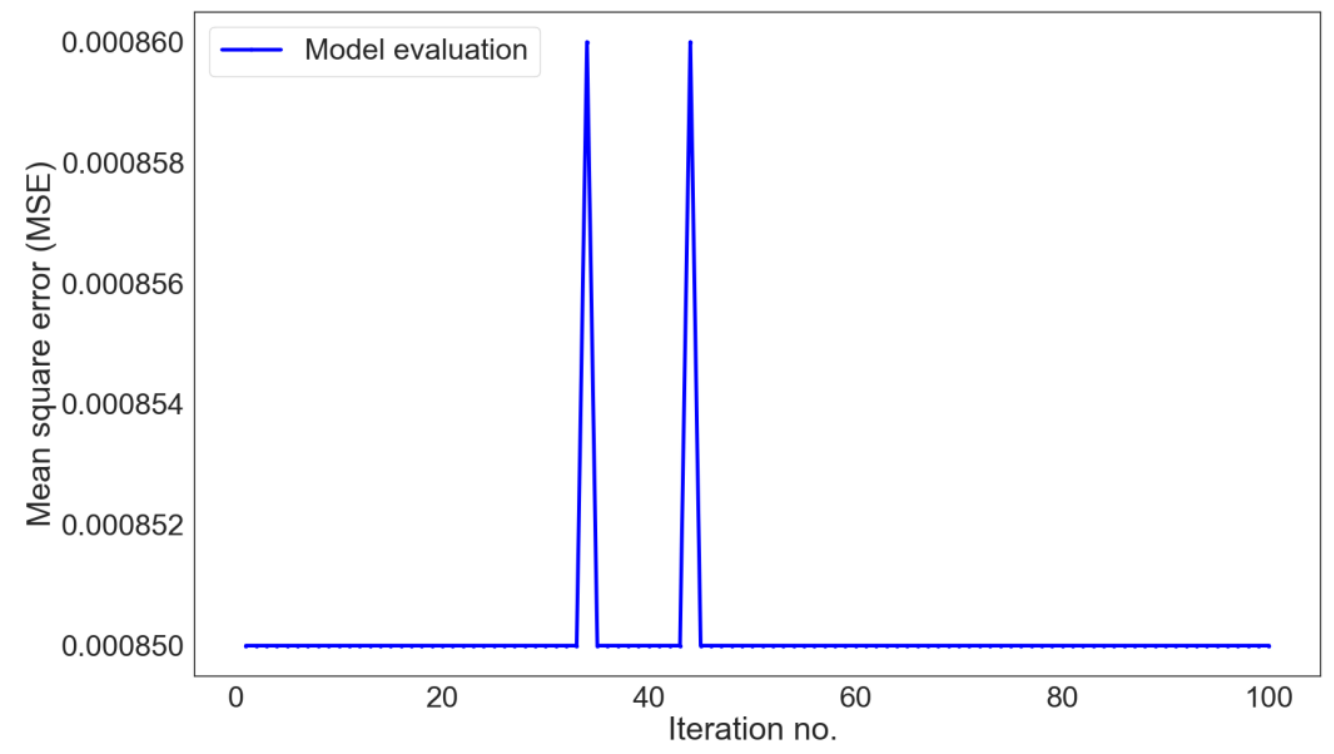

Figure 5. 5 Scikit-learn library-based MSE (with respect to different randomized sampling iterations), while predicting strain for first 50 cycles of ET-F54 test case.

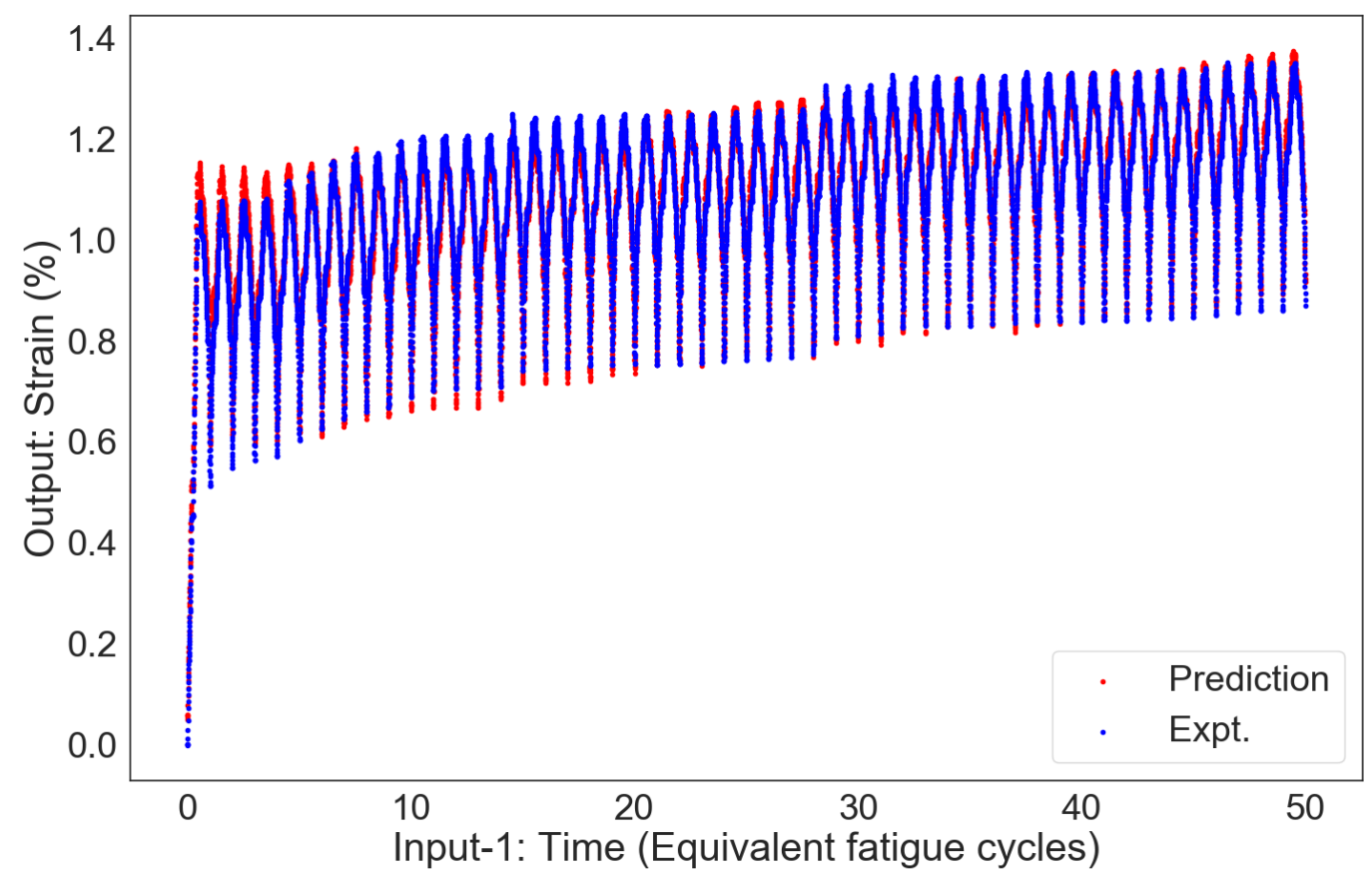

Figure 5. 6 Scikit-learn library-based predicted strain (for first 50 cycles of ET-F54 test case) and its comparison to corresponding experimental strain. 


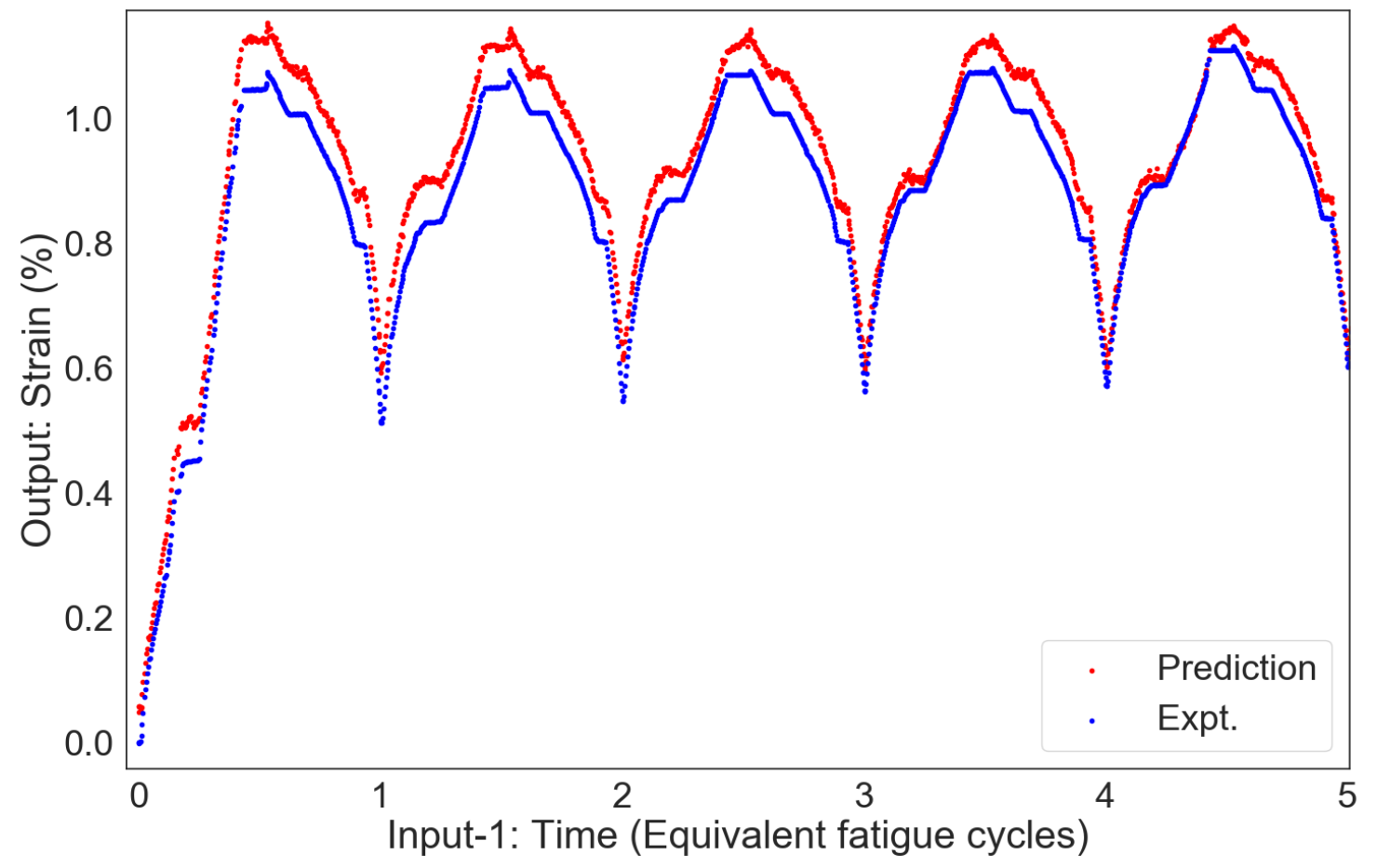

Figure 5. 7 Magnified version of Figure 5.6.

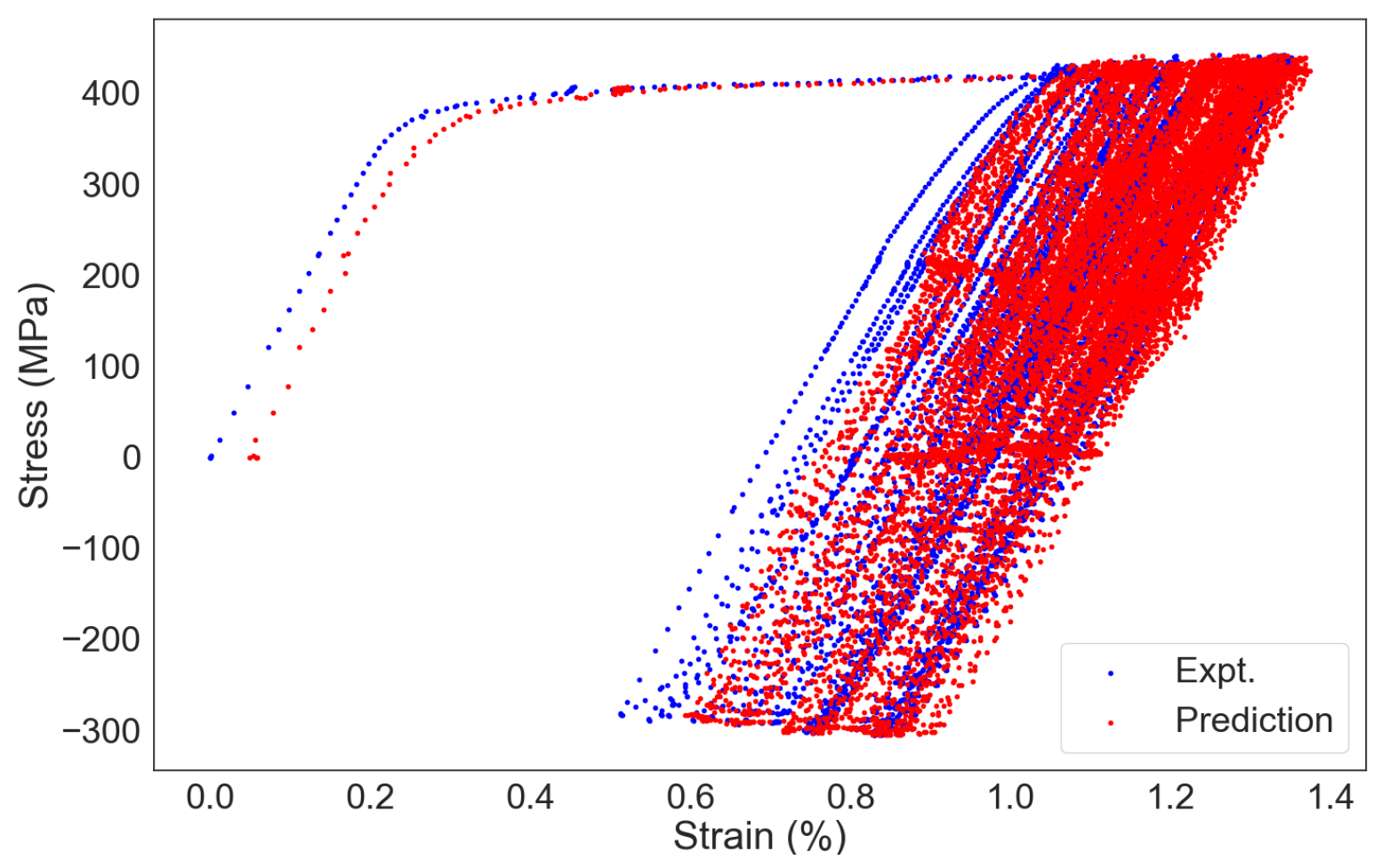

Figure 5. 8 Scikit-learn library-based predicted strain hysteresis curves (for first 50 cycles of ET-F54 test case) and its comparison to corresponding experimental strain hysteresis curves. 
A Hybrid Al/ML and Computational Mechanics Based Approach for Time-Series State and Fatigue Life Estimation of Nuclear Reactor Components

5.1.2.2 Cyclic strain estimation using AI/DL based Keras library and Sigmoid activation function

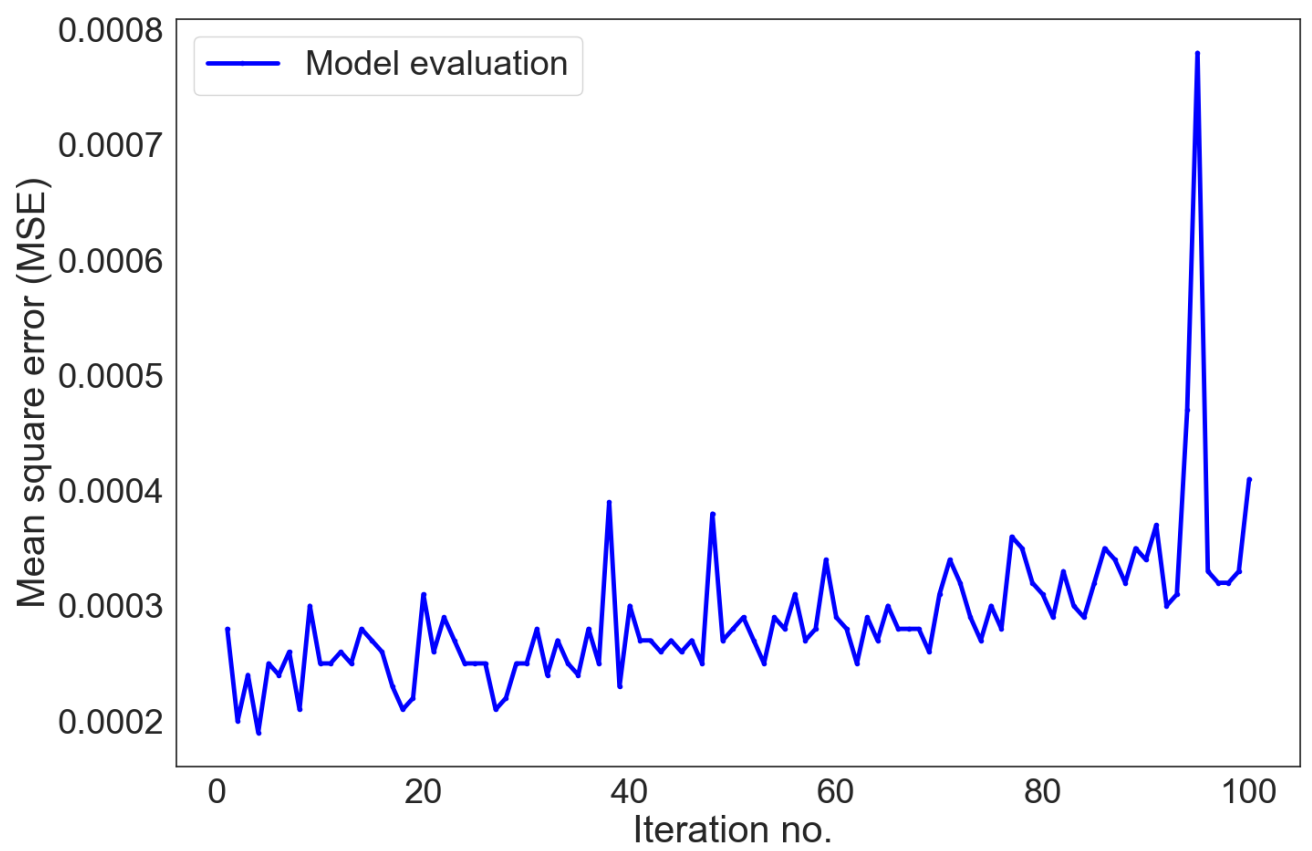

Figure 5. 9 Keras library and Sigmoid activation-function based MSE (with respect to different randomized sampling iterations), while predicting strain for first 50 cycles of ET-F54 test case.

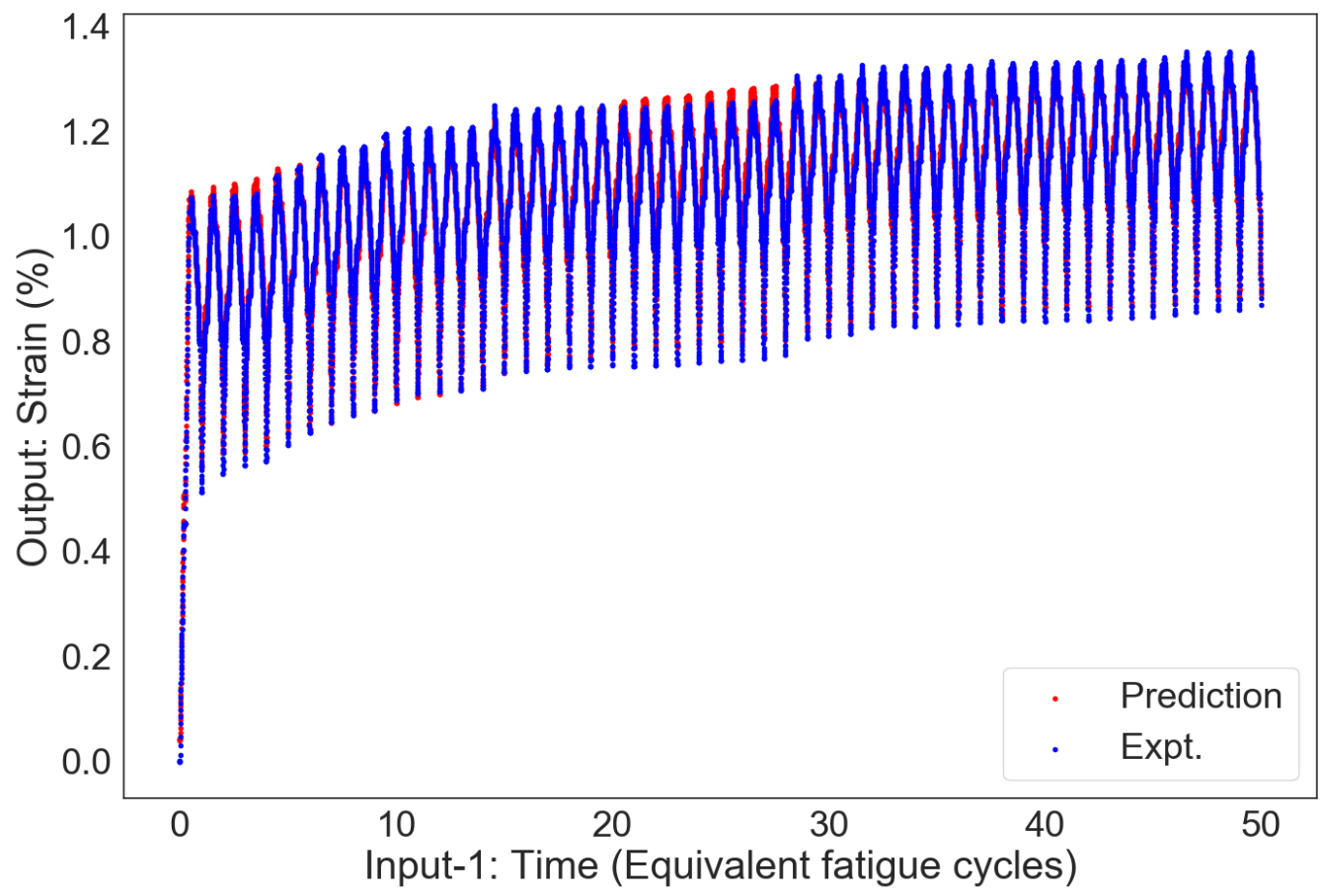

Figure 5. 10 Keras library and Sigmoid activation-function based predicted strain (for first 50 cycles of ET-F54 test case) and its comparison to corresponding experimental strain. 


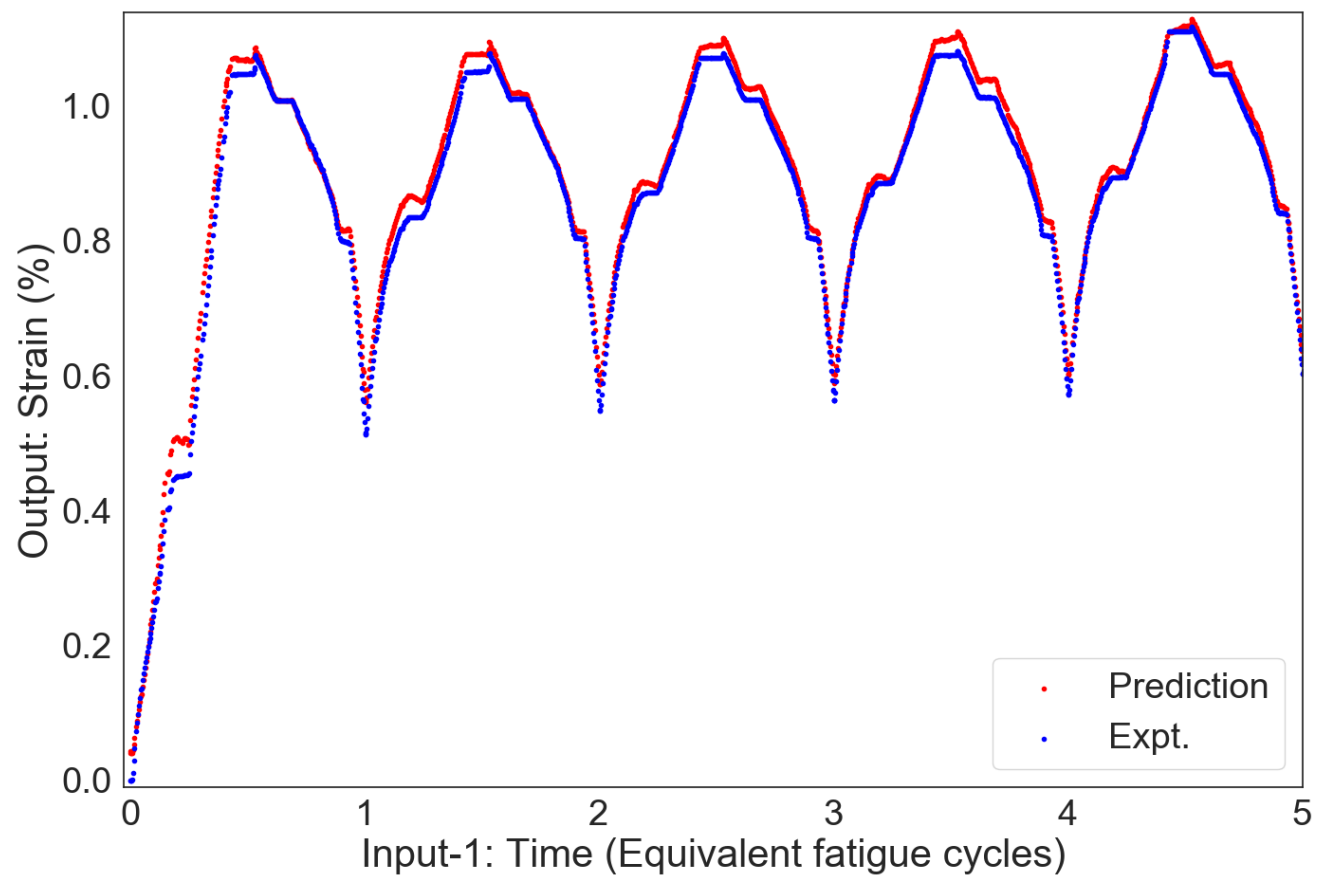

Figure 5. 11 Magnified version of Figure 5.10.

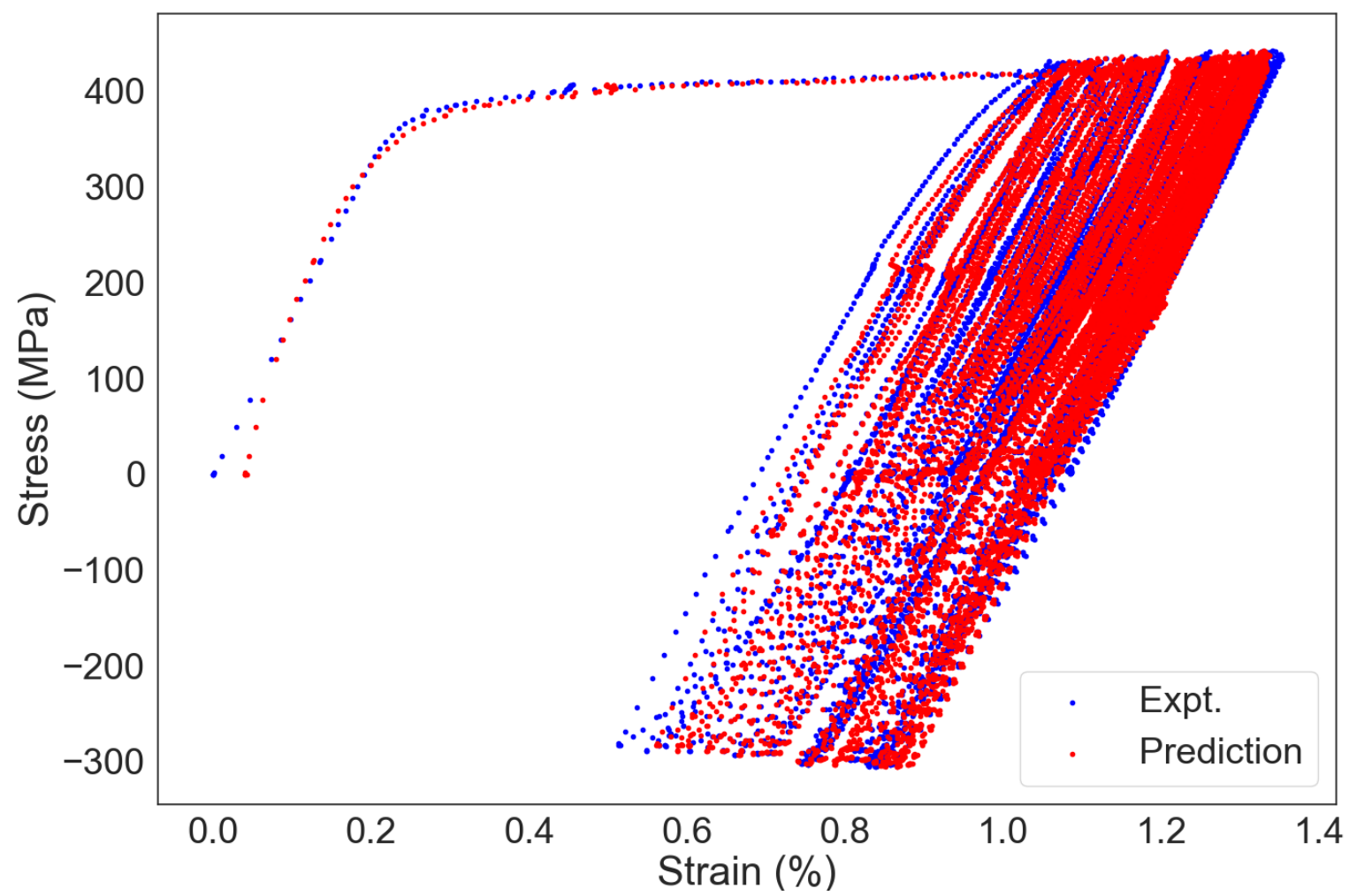

Figure 5. 12 Keras library and Sigmoid activation-function based hysteresis curves (for first 50 cycles of ET-F54 test case) and its comparison to corresponding experimental strain hysteresis curves. 
5.1.2.3 Cyclic strain estimation using AI/DL based Keras library and ReLU activation function

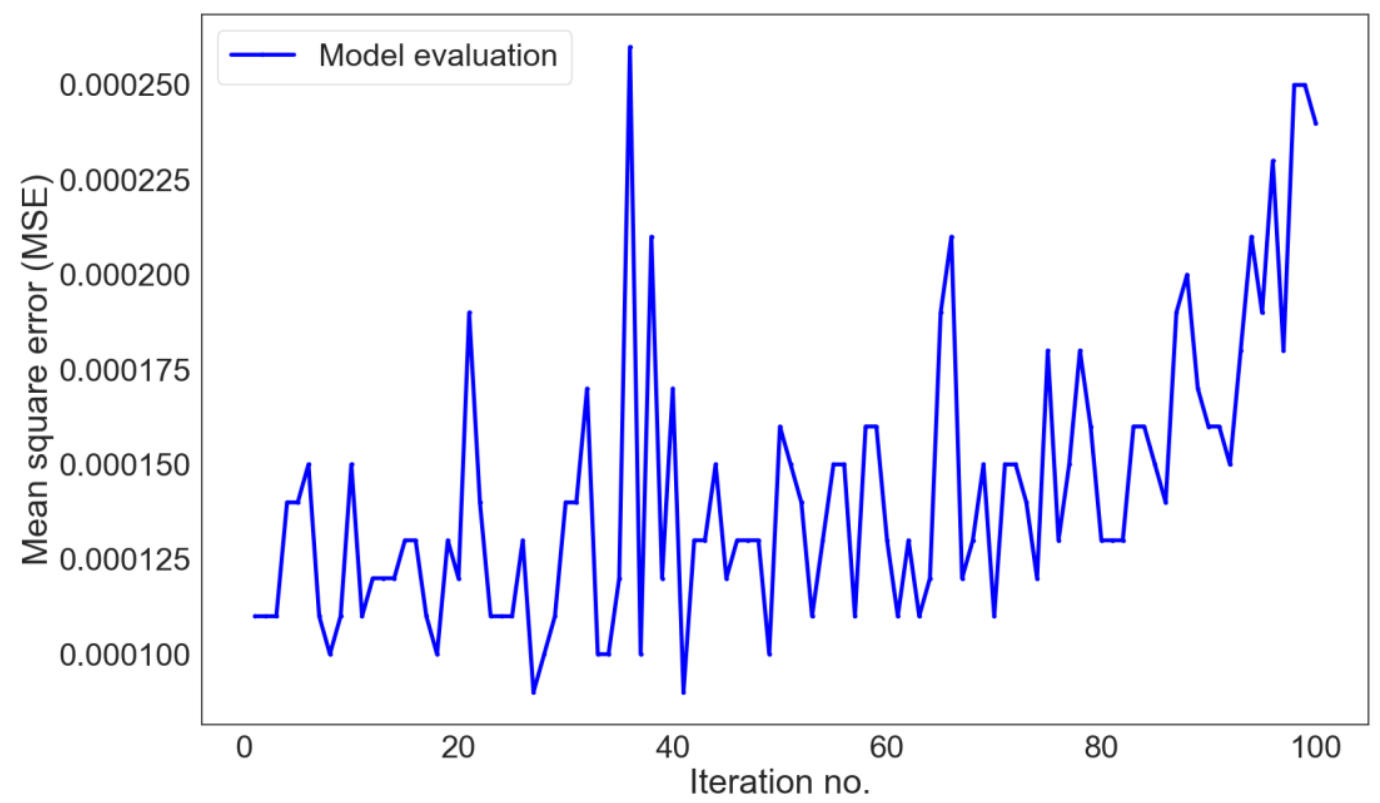

Figure 5. 13 Keras library and ReLU activation-function based MSE (with respect to different randomized sampling iterations), while predicting strain for first 50 cycles of ET-F54 test case.

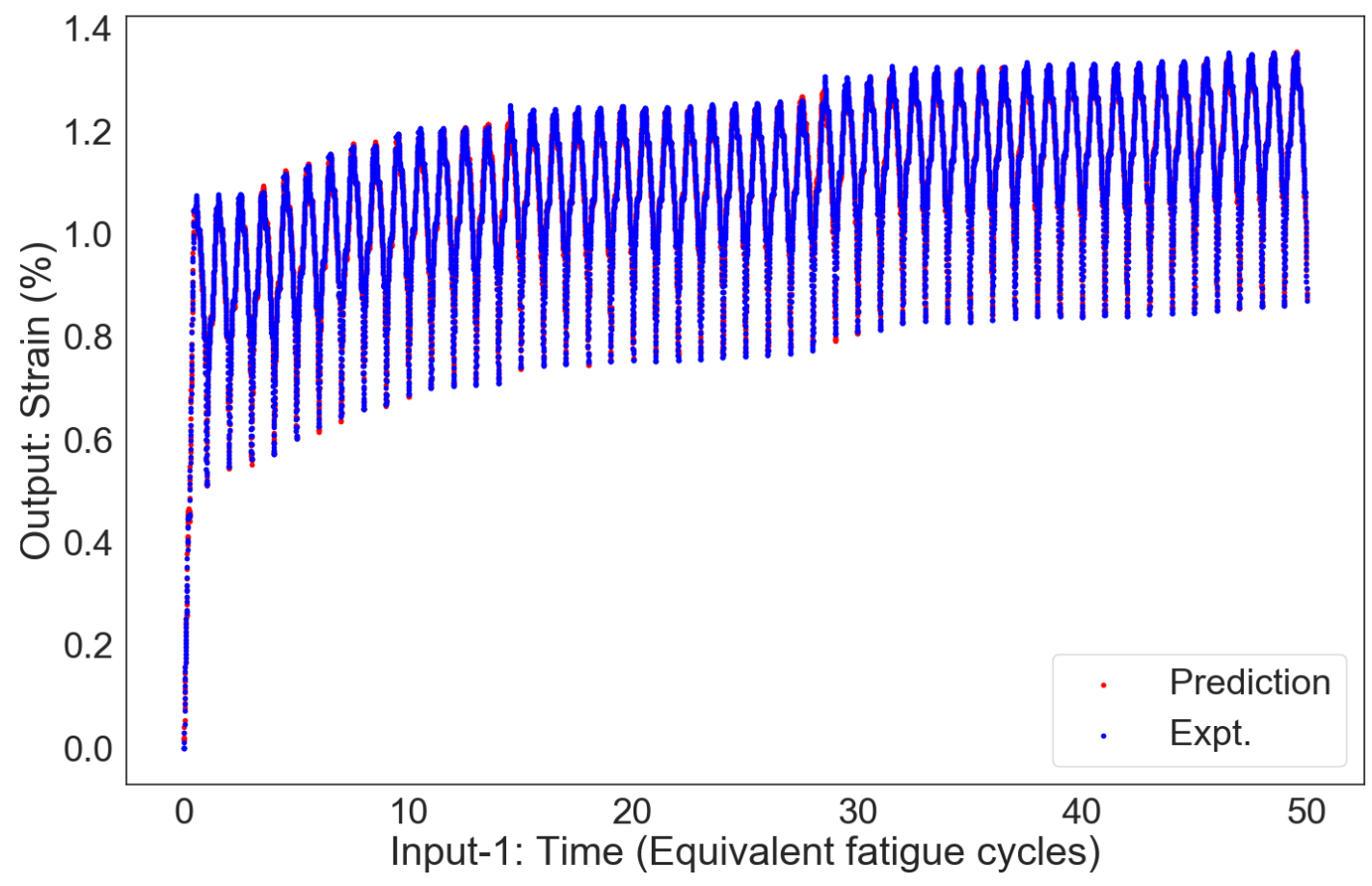

Figure 5. 14 Keras library and ReLU activation-function based predicted strain (for first 50 cycles of ETF54 test case) and its comparison to corresponding experimental strain. 


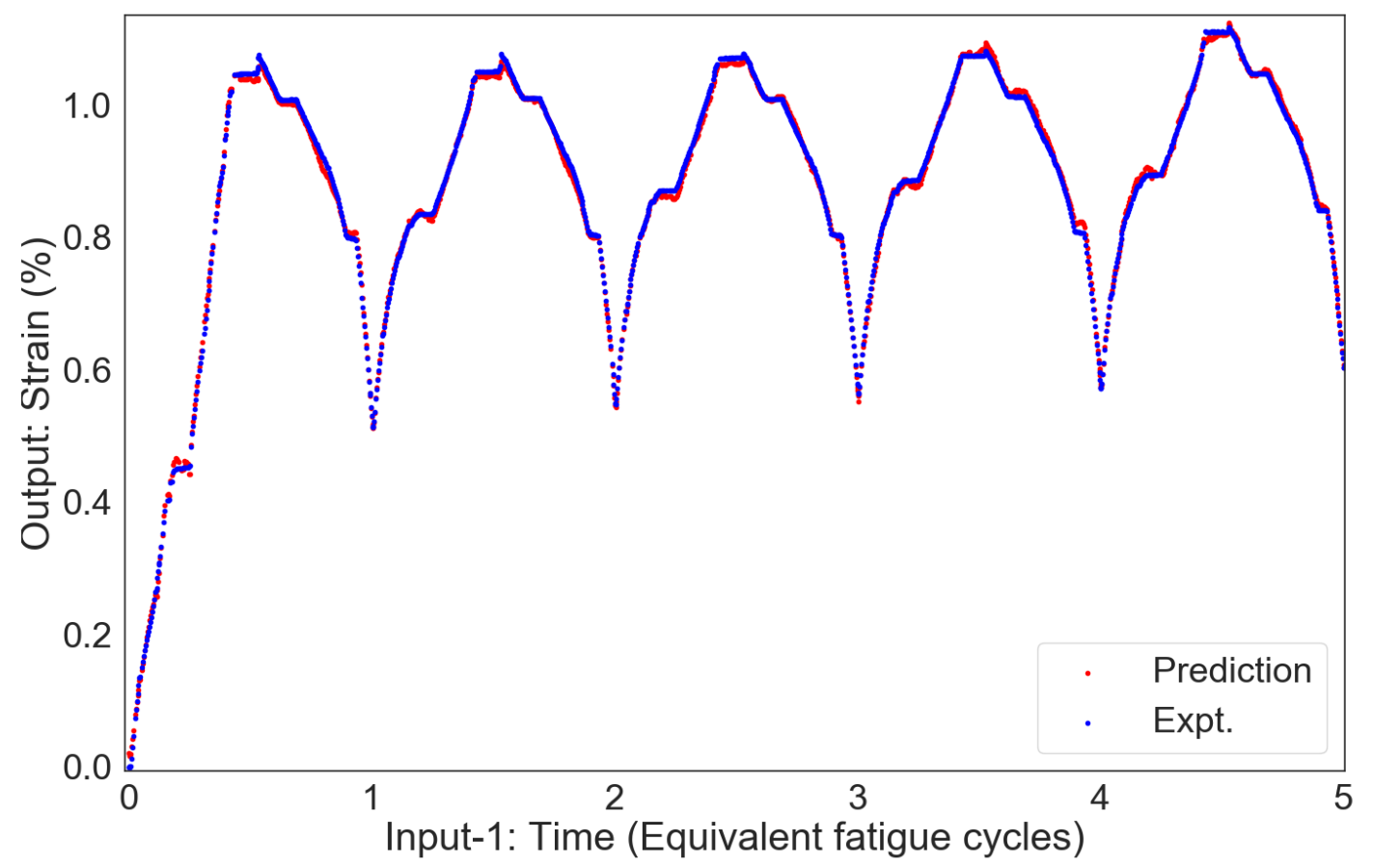

Figure 5. 15 Magnified version of Figure 5.14.

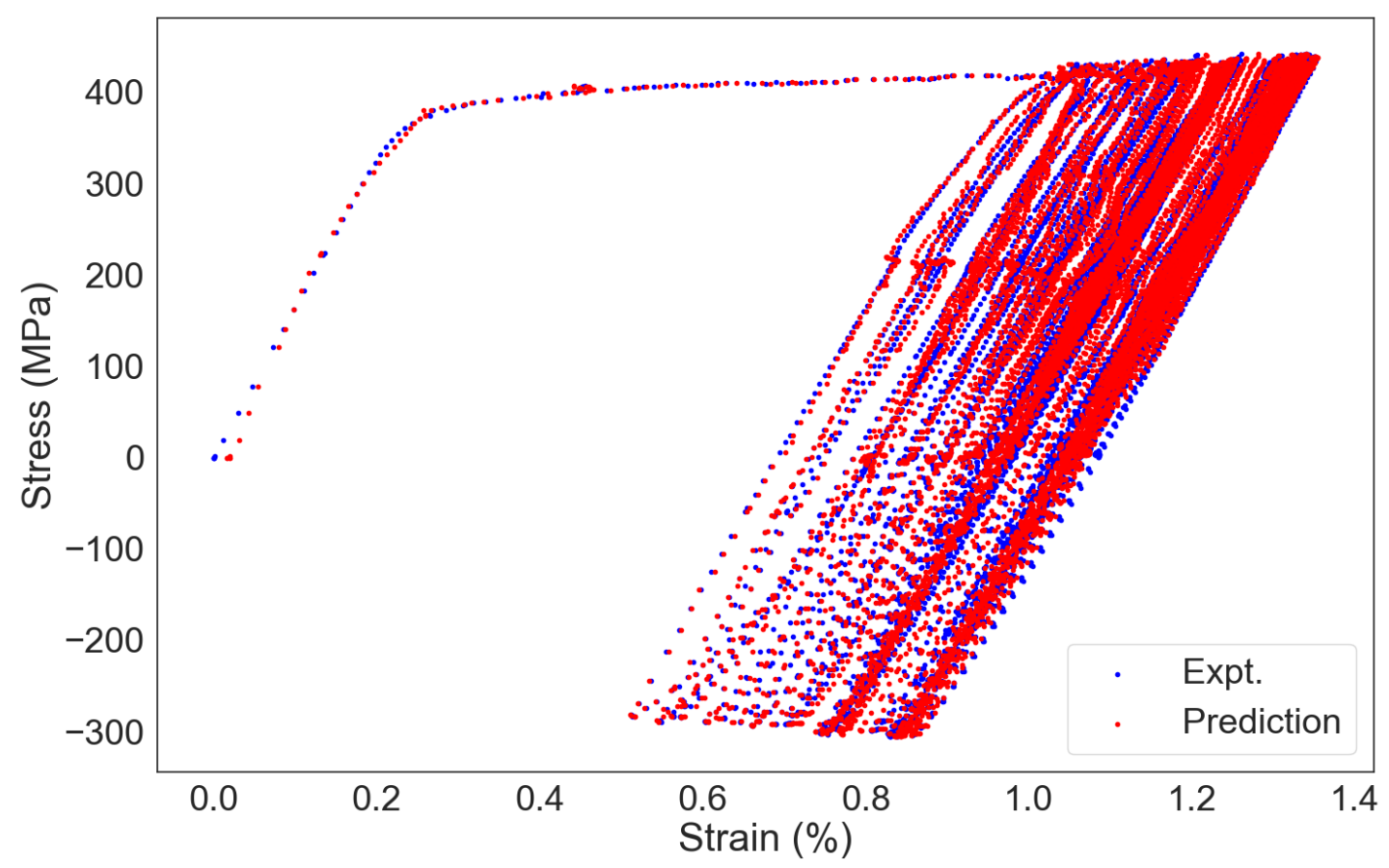

Figure 5. 16 Keras library and ReLU activation-function based hysteresis curves (for first 50 cycles of ET-F54 test case) and its comparison to corresponding experimental strain hysteresis curves. 


\subsubsection{Clustering and piecewise strain time-series estimation to deal with non-stationarity associated with cyclic hardening under unsymmetrical design-basis cyclic loading}

Fatigue data often follows nonstationary probability distribution due to cyclic hardening and softening. The cyclic hardening and softening lead to cyclic shifting of mean and variance of target observations (here the strain). The varying mean and variance (or standard deviation) can lead to erroneous prediction results if a single set of parameters used for modeling the entire fatigue life with thousands of fatigue cycles. To address this issue, we used Scikit-learn based k-mean clustering technique (refer to section 2.6) to cluster/group the entire fatigue life to multiple subdomains. Based on the individual subdomain parameters we predicted the strain of that subdomain. We used the Kreas DL library with ReLU activation function to model and predict the time-series strain data for entire fatigue life of ET-F54 fatigue case. Below show the related results. For example, Figures 5.17 and 5.18 show the variation of mean and standard deviation of cyclic strain. These results show that the whole-life fatigue data follows a nonstationary probability distribution. Figures 5.19 and 5.20 show the related results for clustering/grouping the entire fatigue life of ET-F54 specimen into multiple subdomains. Figures 5.21 to 5.25 show the corresponding Kreas model predicted results. Note that the mean square error (MSE) based prediction accuracy (based on the mean square error estimation) for the entire fatigue life was found to be 0.0001 . The estimated MSE for the whole-life prediction is even lesser compared to the Keras/ReLU based 50 cycle case prediction error (MSE of 0.00026, refer to Table 5.1). These results show the importance of piecewise prediction through appropriate clustering of the entire fatigue life.

\subsubsection{Non-stationarity process associated with cyclic hardening}

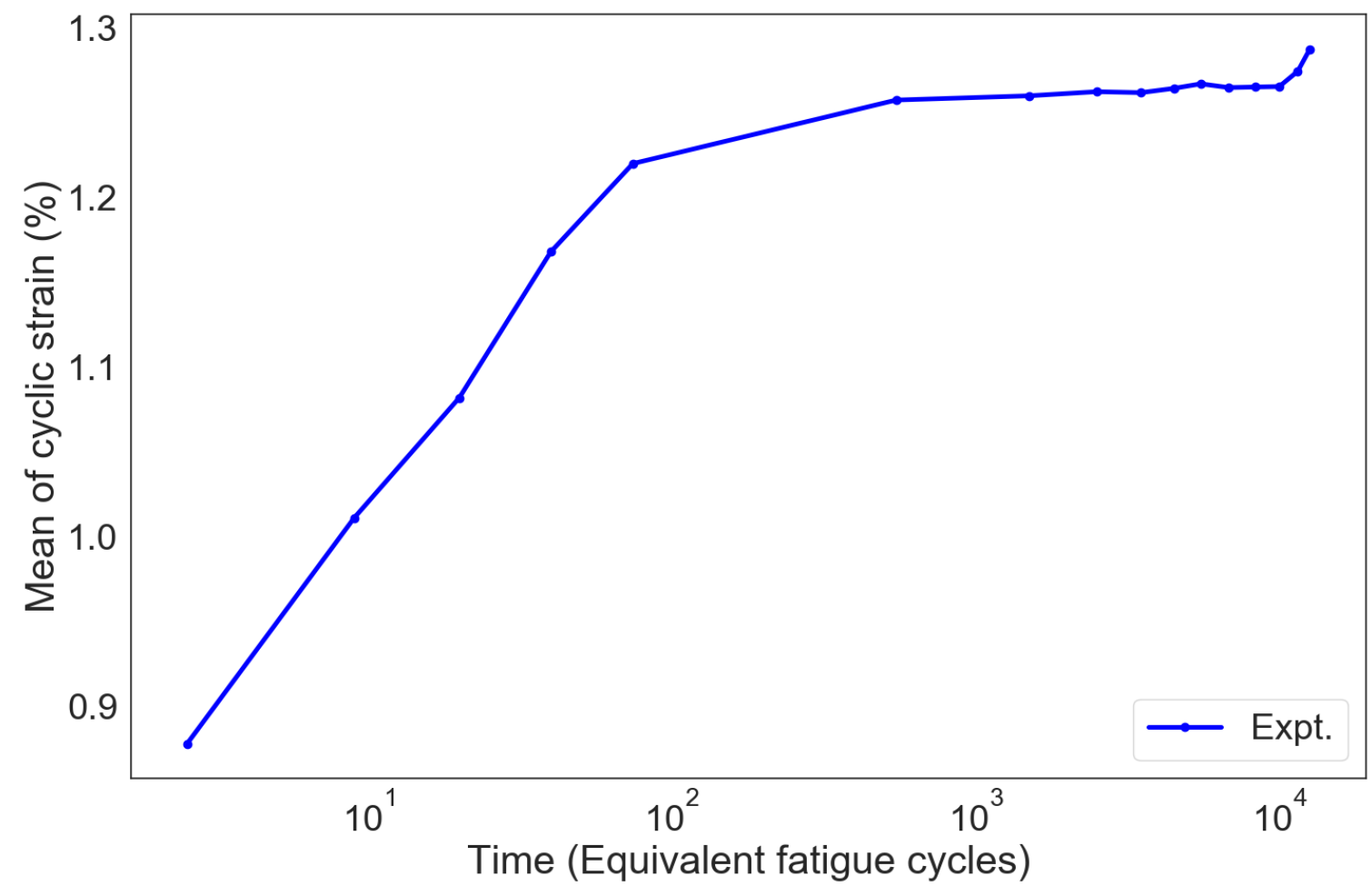

Figure 5. 17 Substantial variation of mean of ET-F54 cyclic strains shows that the time-series strain for the entire fatigue life follows a nonstationary process. 


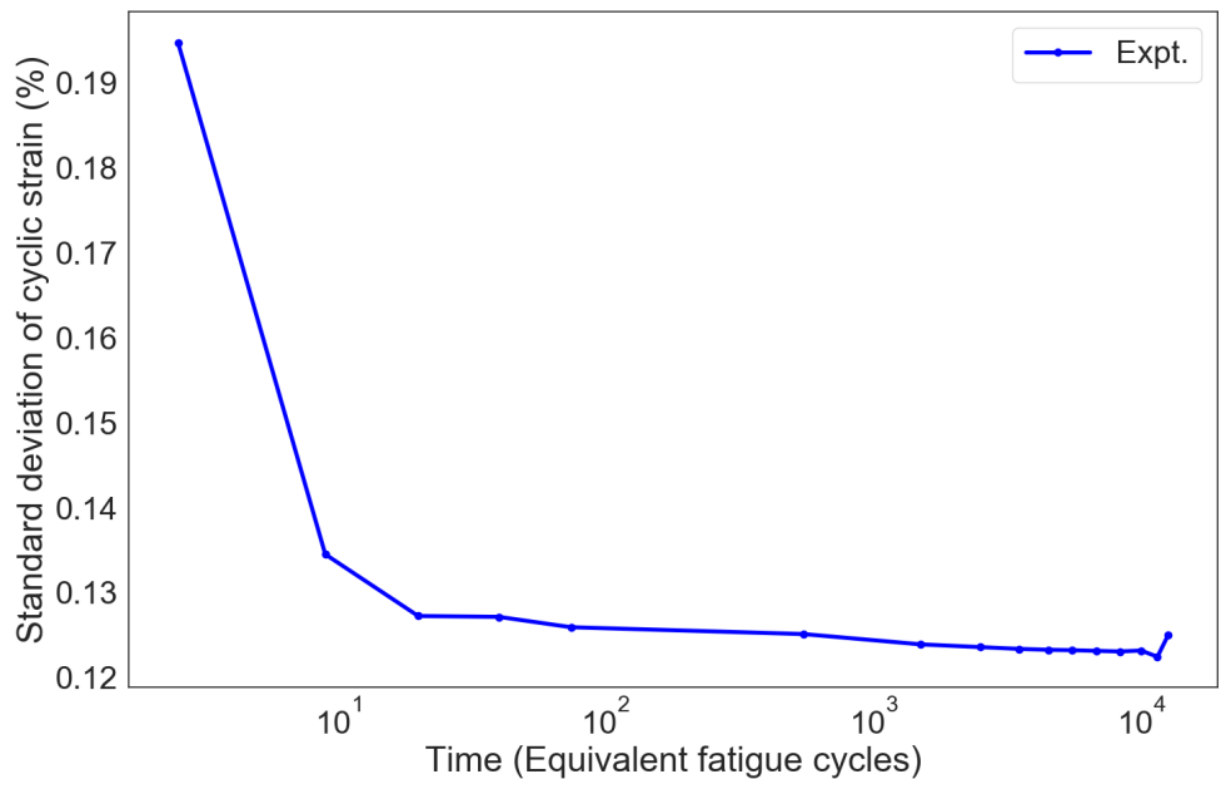

Figure 5. 18 Substantial variation of standard deviation of ET-F54 cyclic strains shows that the timeseries strain for the entire fatigue life follows a nonstationary process.

5.1.3.2 Scikit-learn library-based K-Mean clustering to divide the entire fatigue life to multiple subdomains

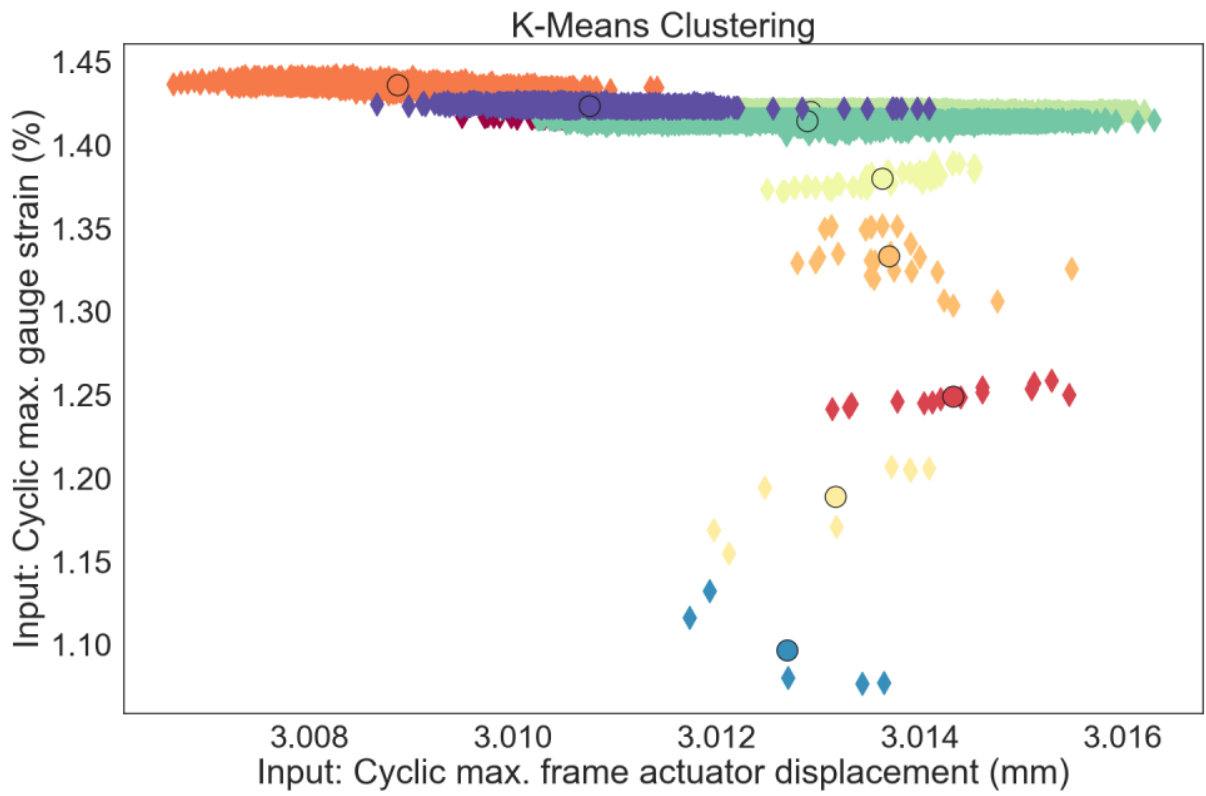

Figure 5. 19 ET-F54 clustered data showing the 2-dimensional dependency between frame maximum actuator displacement and corresponding specimen gage area maximum strain. Raw data in an individual cluster (plotted with a single color) are reparented by dots whereas, the solid circles represent the corresponding cluster center. 


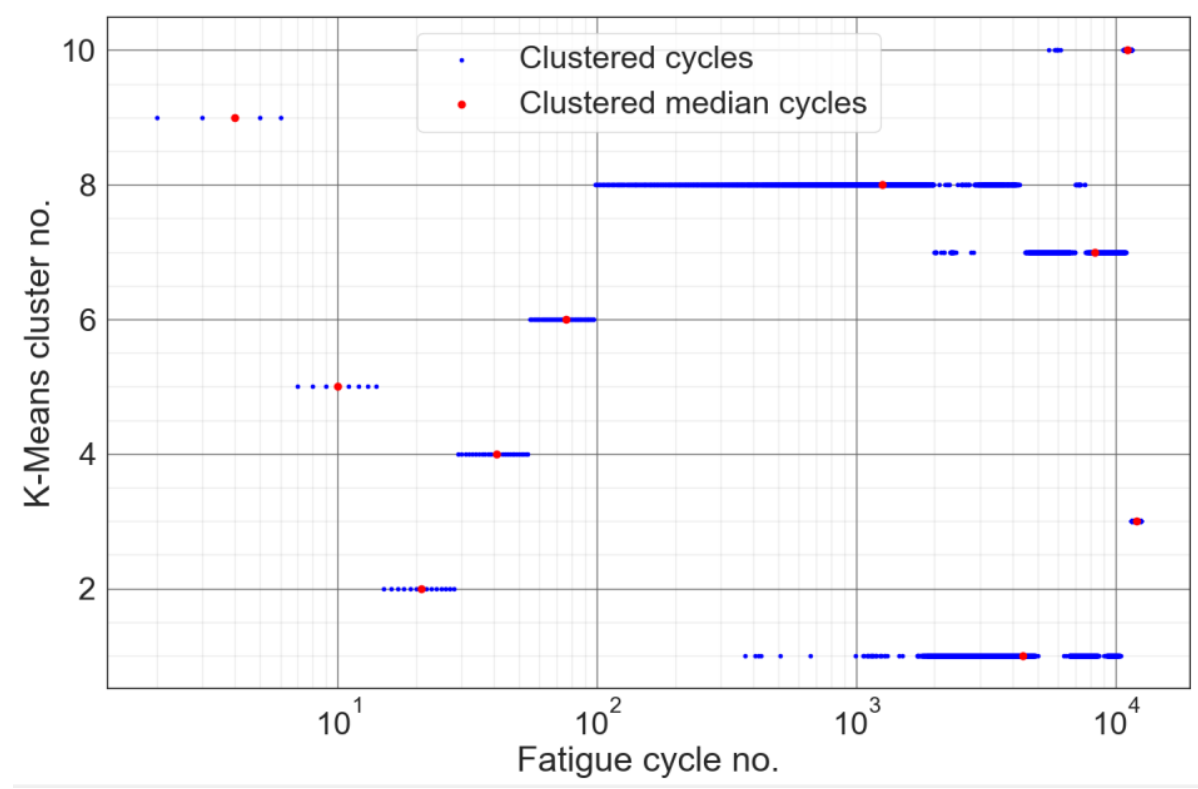

Figure 5. 20 Cycle versus corresponding cluster numbers showing how the entire ET-F54 fatigue-test data clustered into only 10 different groups/clusters. Piecewise strain prediction models were developed for individual clusters to predict the strain for the entire fatigue life of ET-F54 test case.

5.1.3.3 Piecewise strain prediction for the entire fatigue life of ET-F54 specimen using AI/DL based Keras library and ReLU activation function

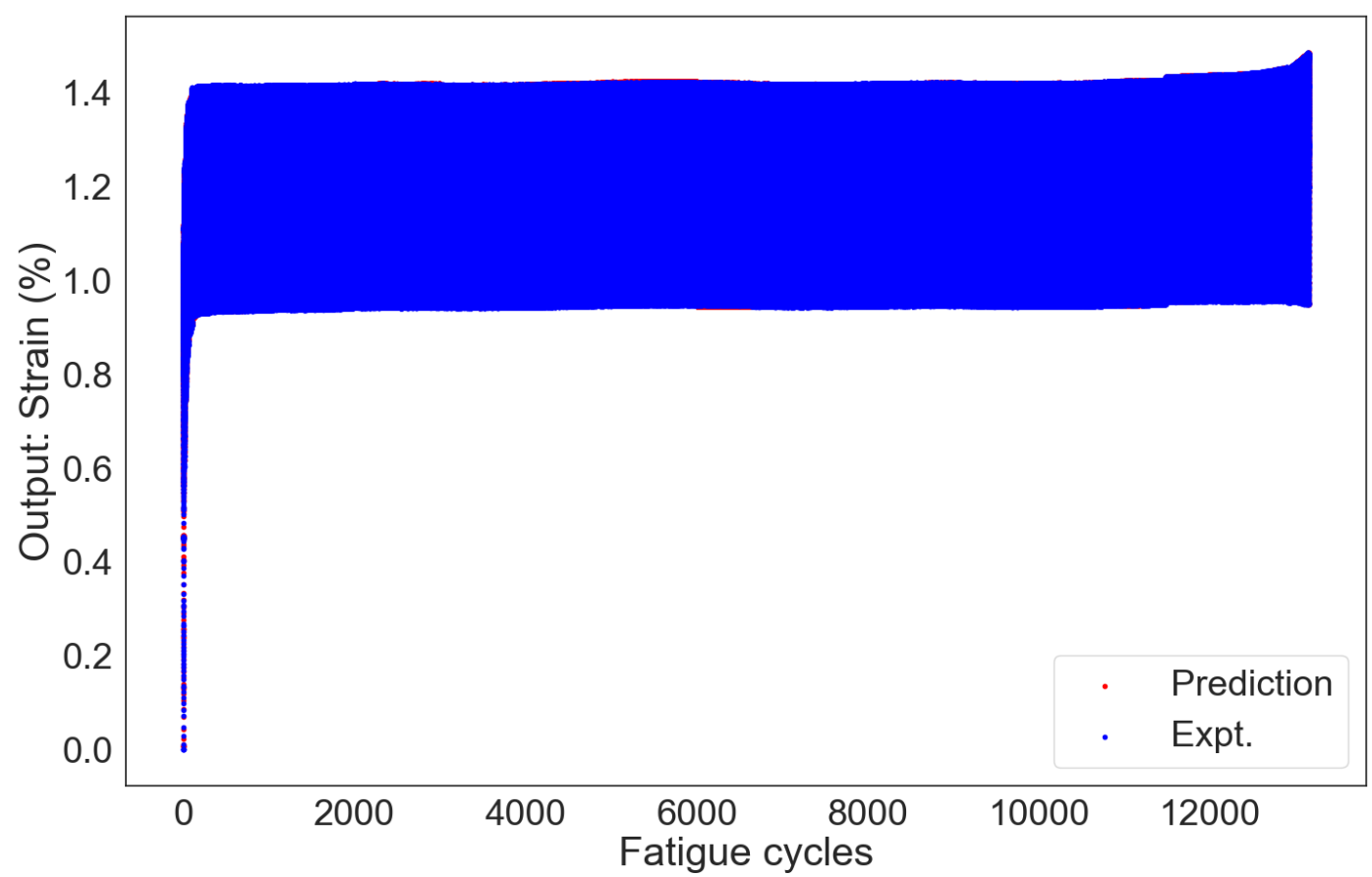

Figure 5. 21 Keras library and ReLU activation-function based predicted time-series strain (for the entire fatigue cycles of ET-F54 test case) and its comparison to corresponding experimental strain. 


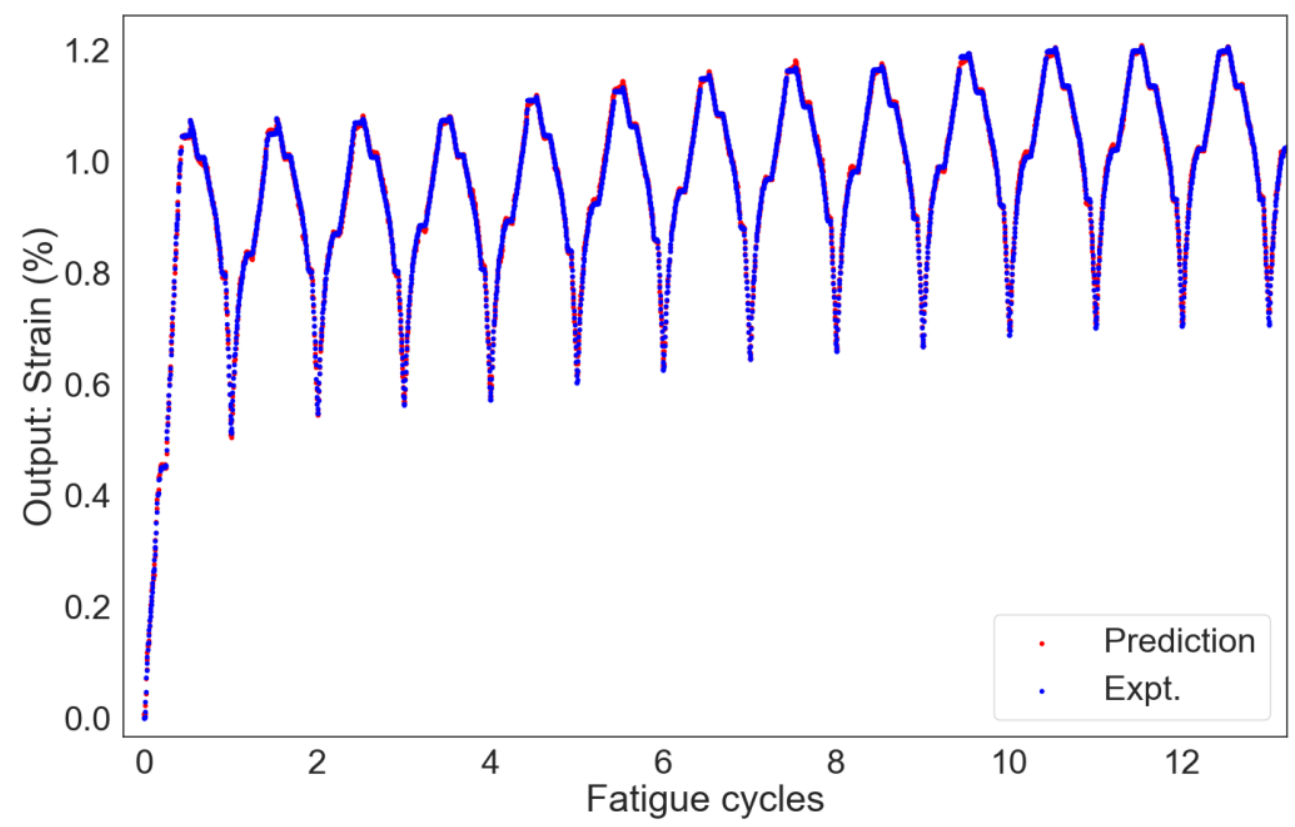

Figure 5. 22 Magnified version of Figure 5.21 showing first few cycles (of ET-F54 test case) prediction versus experimental results.

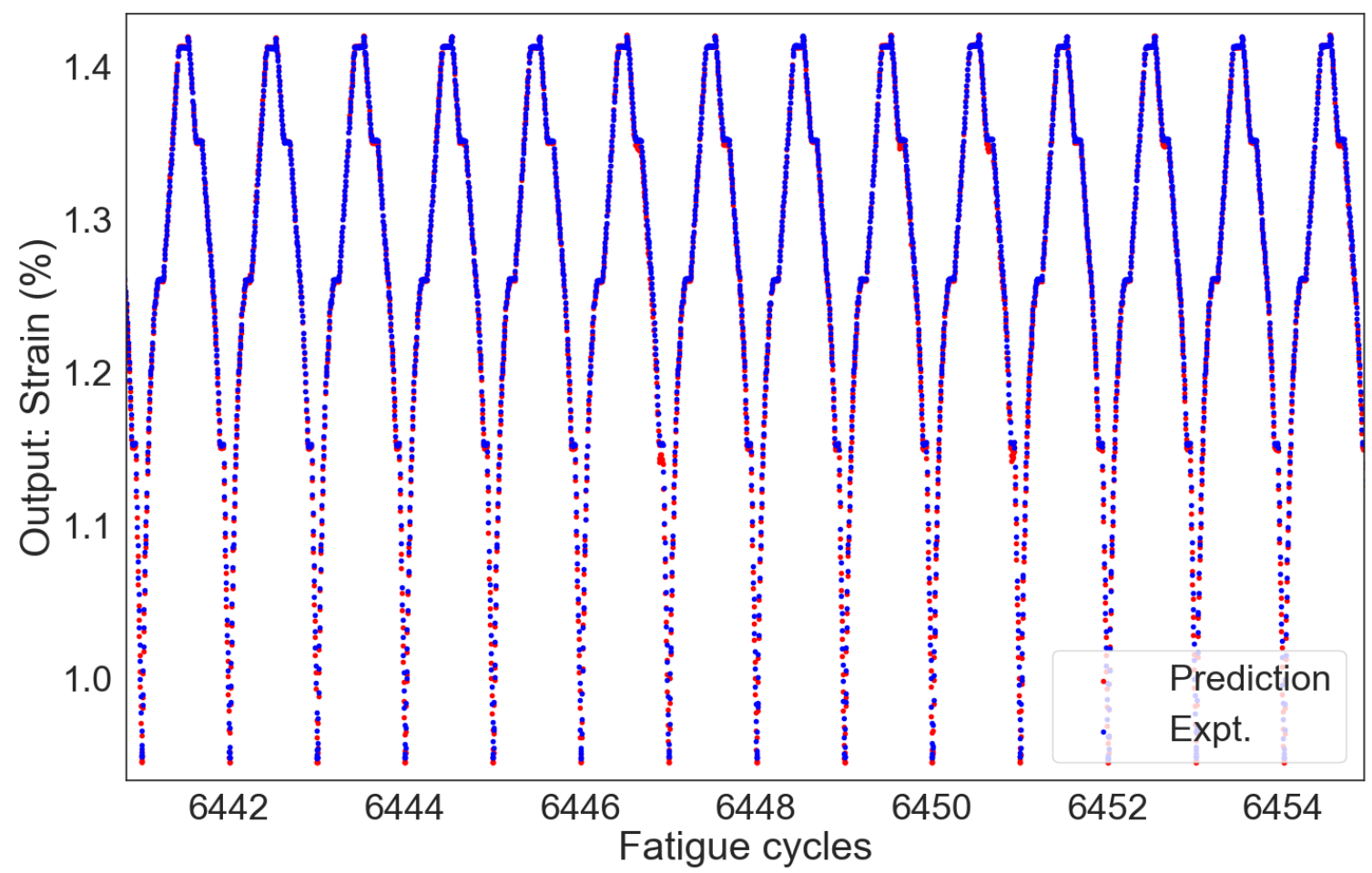

Figure 5. 23 Magnified version of Figure 5.21 showing few half-life cycles (of ET-F54 test case) prediction versus experimental results. 


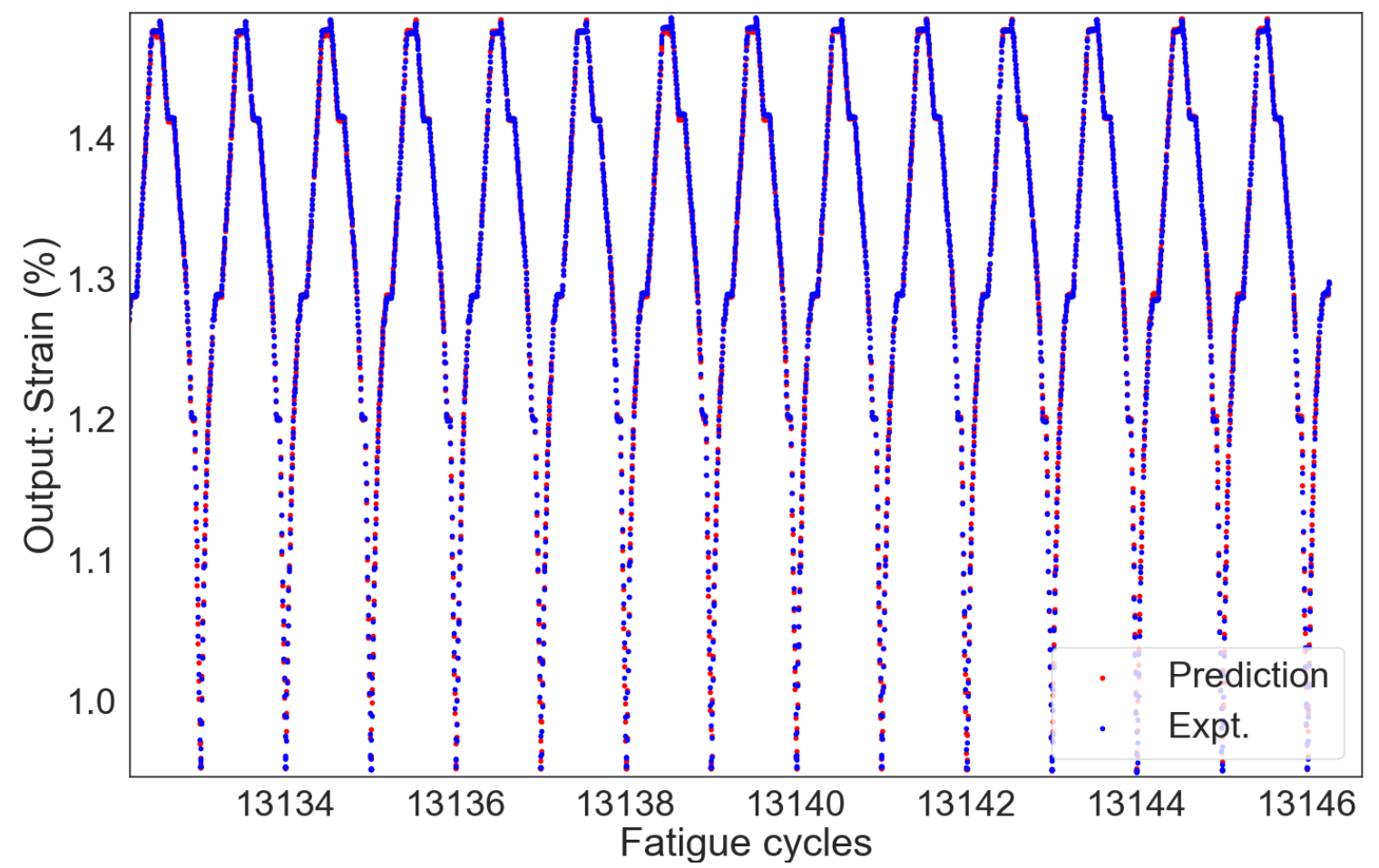

Figure 5. 24 Magnified version of Figure 5.21 showing few end-life cycles (of ET-F54 test case) prediction versus experimental results.

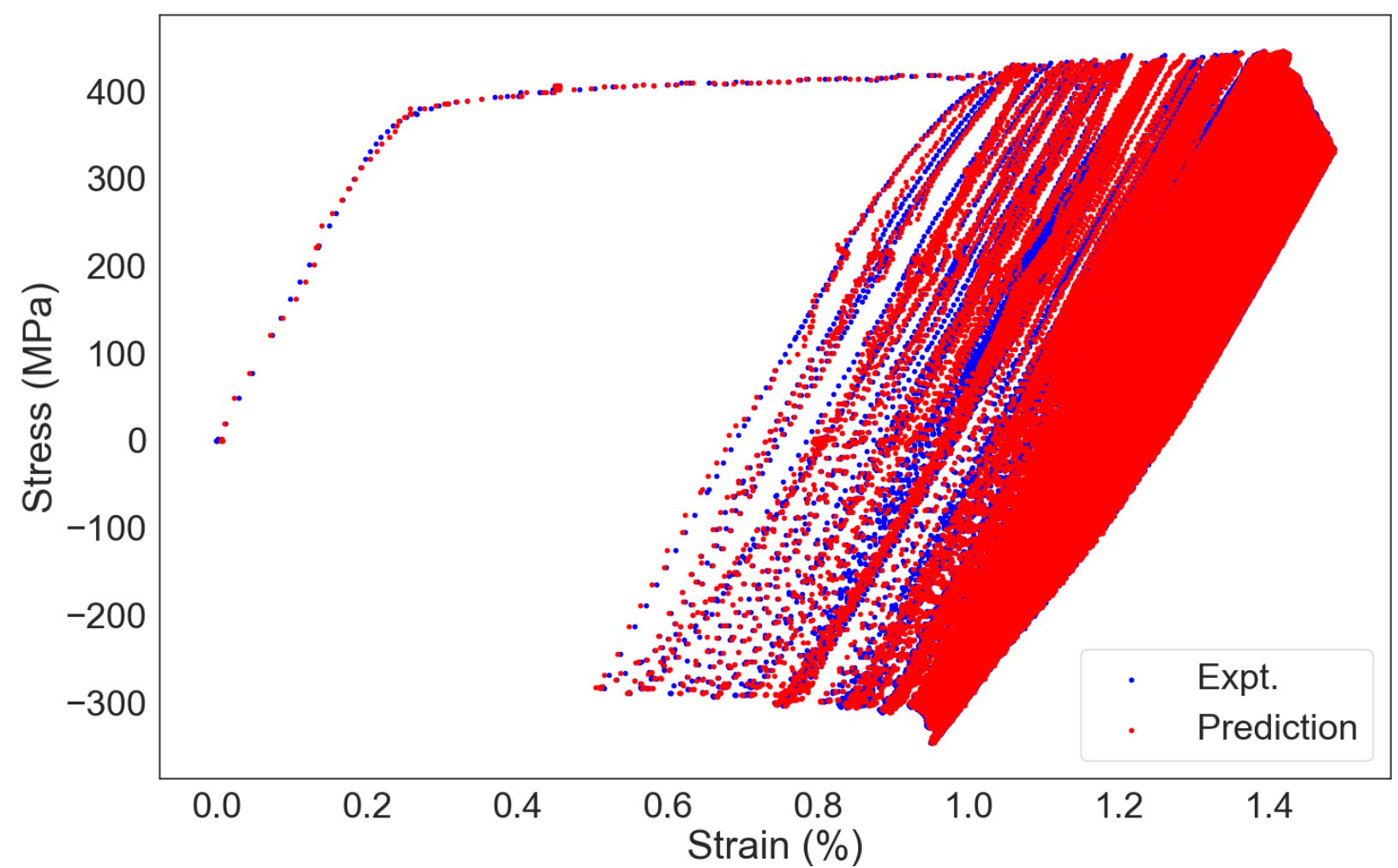

Figure 5. 25 Predicted strain hysteresis curves (for entire fatigue life of ET-F54 test case) and its comparison to corresponding experimental strain hysteresis curves. 


\subsection{Validation case-2: Cyclic Strain Estimation for Fatigue Test Specimen Subjected to ET-F61 Symmetrical Cyclic Loading}

Similar as the validation under unsymmetrical design-base loading case, we also checked the accuracy of the different AI/ML/DL models for symmetrical cyclic loading case. Note that unsymmetrical loading cases are more realistic (as observed in real reactor component). However, symmetrical loading cases are widely followed and suggested by ASME to conduct laboratory based low-cycle-fatigue testing. So, it is essential, we check the proposed AI/ML/DL based modeling techniques for both unsymmetrical and symmetrical loading cases. For the purpose, we tested the in-air test-based ET-F61 (refer to section 3 and 4 for test details) fatigue data. The related results are presented below.

\subsubsection{Study the effect of independent variables on accuracy of strain estimation under symmetrical cyclic loading}

The effect of different independent variable (as input dimensions) in the accuracy of the prediction results were tested for the ET-F61 symmetrical loading case. For the purpose we used Keras library with ReLU activation function and first 50 cycle data of ET-F61 DMW specimen. Figures 5.26 to 5.29 show the related results. From these figures, similar as for unsymmetrical loading case single input dimension produces erroneous prediction results. This led us to use multiple dimensions for more accurate prediction. For all the results discussed in the later subsections, we developed the strain prediction models considering 4 independent dimensions such as time, pull-rod-displacement measurements, frame-actuator-displacement measurements and frame-load-cell measurements. 


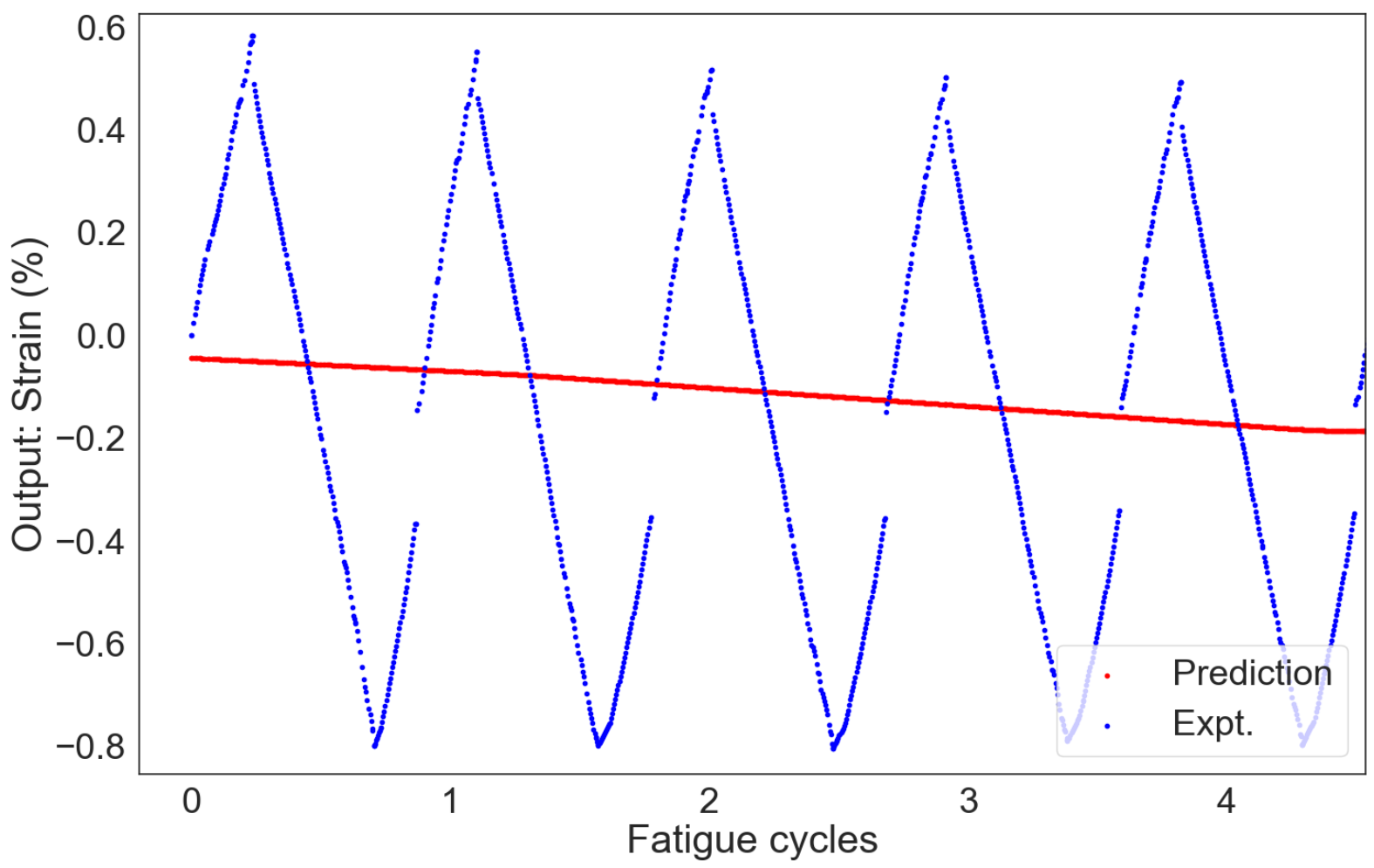

Figure 5.26 Cyclic strain estimation using the time as the only independent variable.

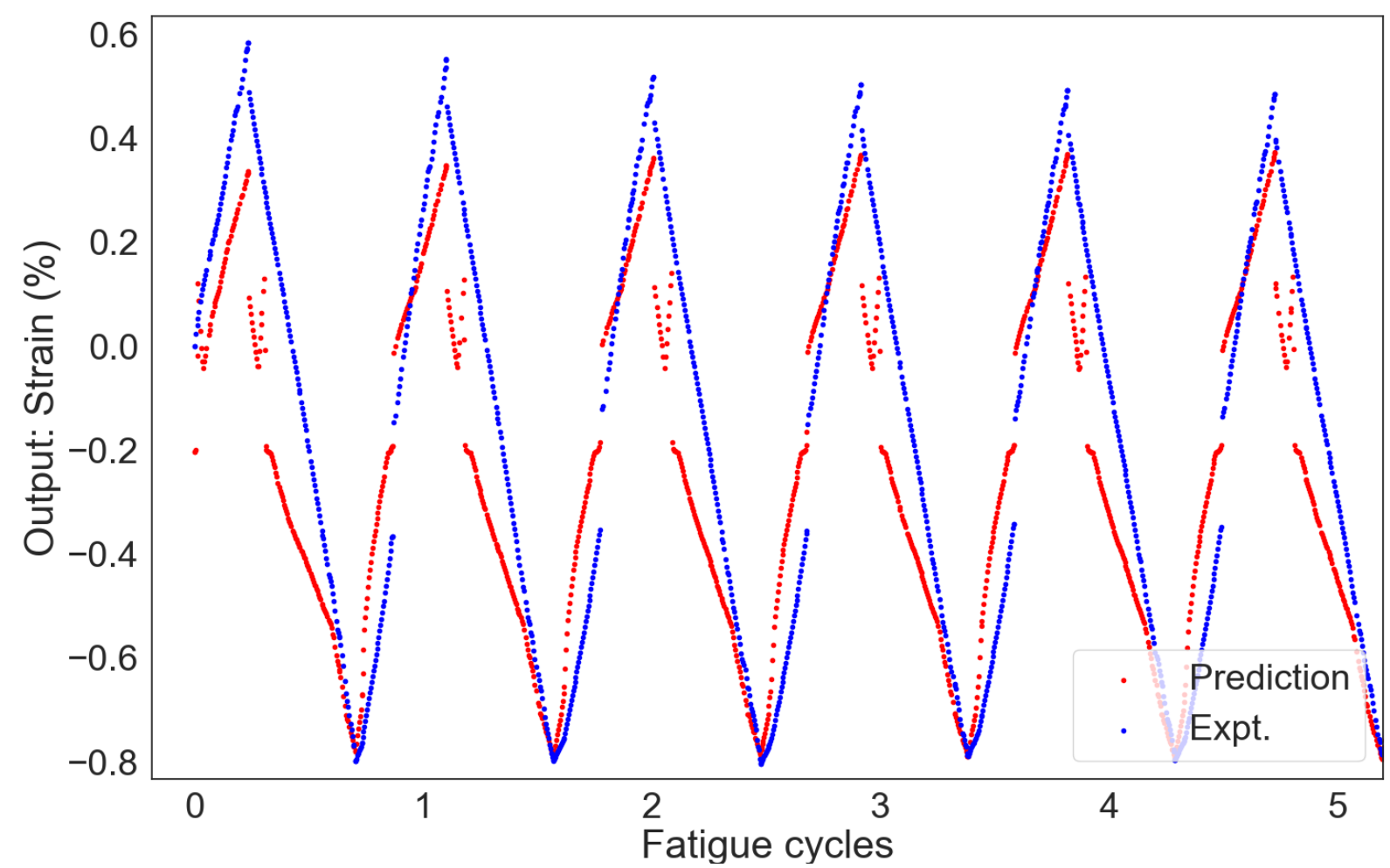

Figure 5.27 Cyclic strain estimation using displacement of the test-frame actuator as the only independent variable. 


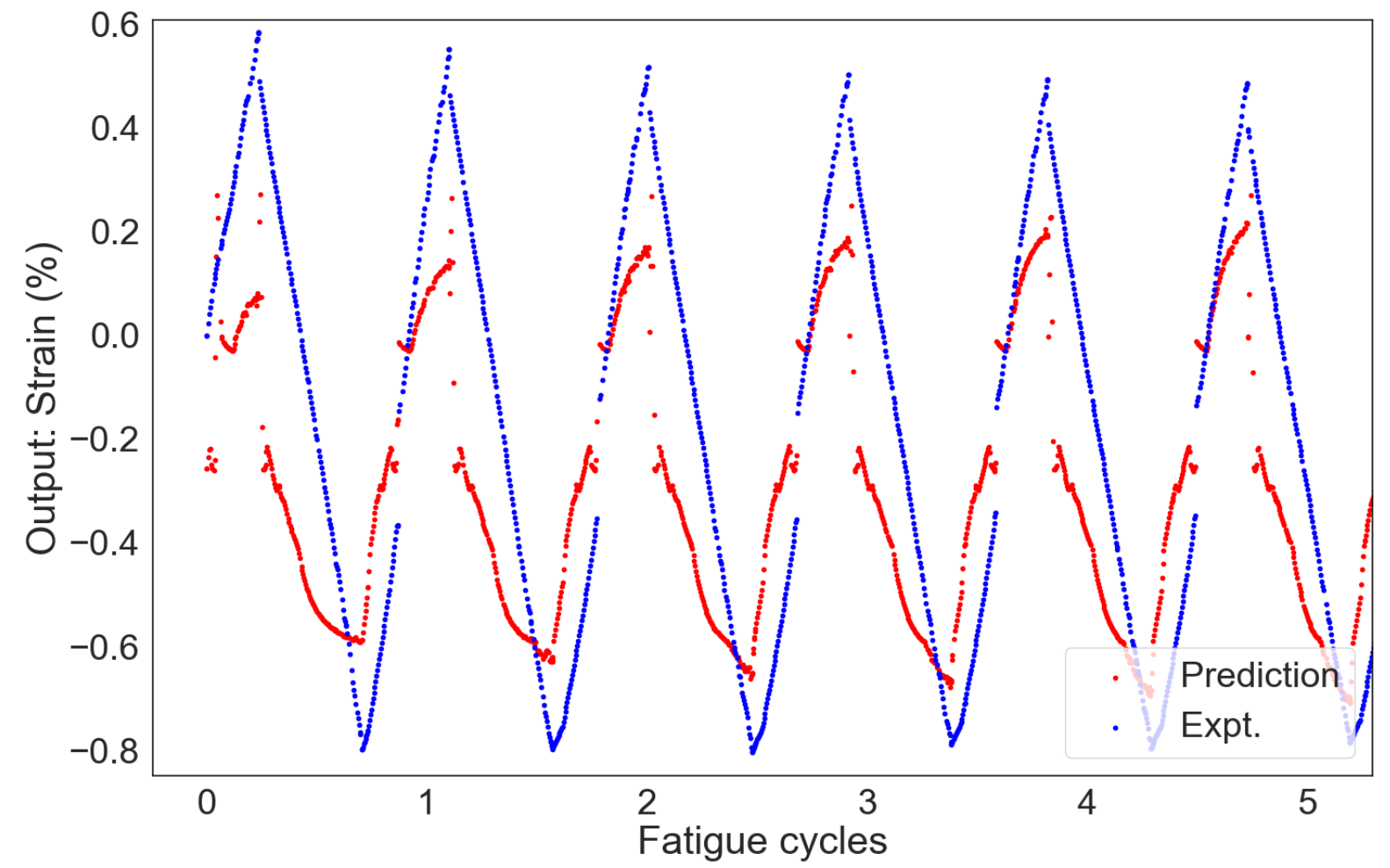

Figure 5.28 Cyclic strain estimation using the load-cell measurement of the test-frame as the only independent variable.

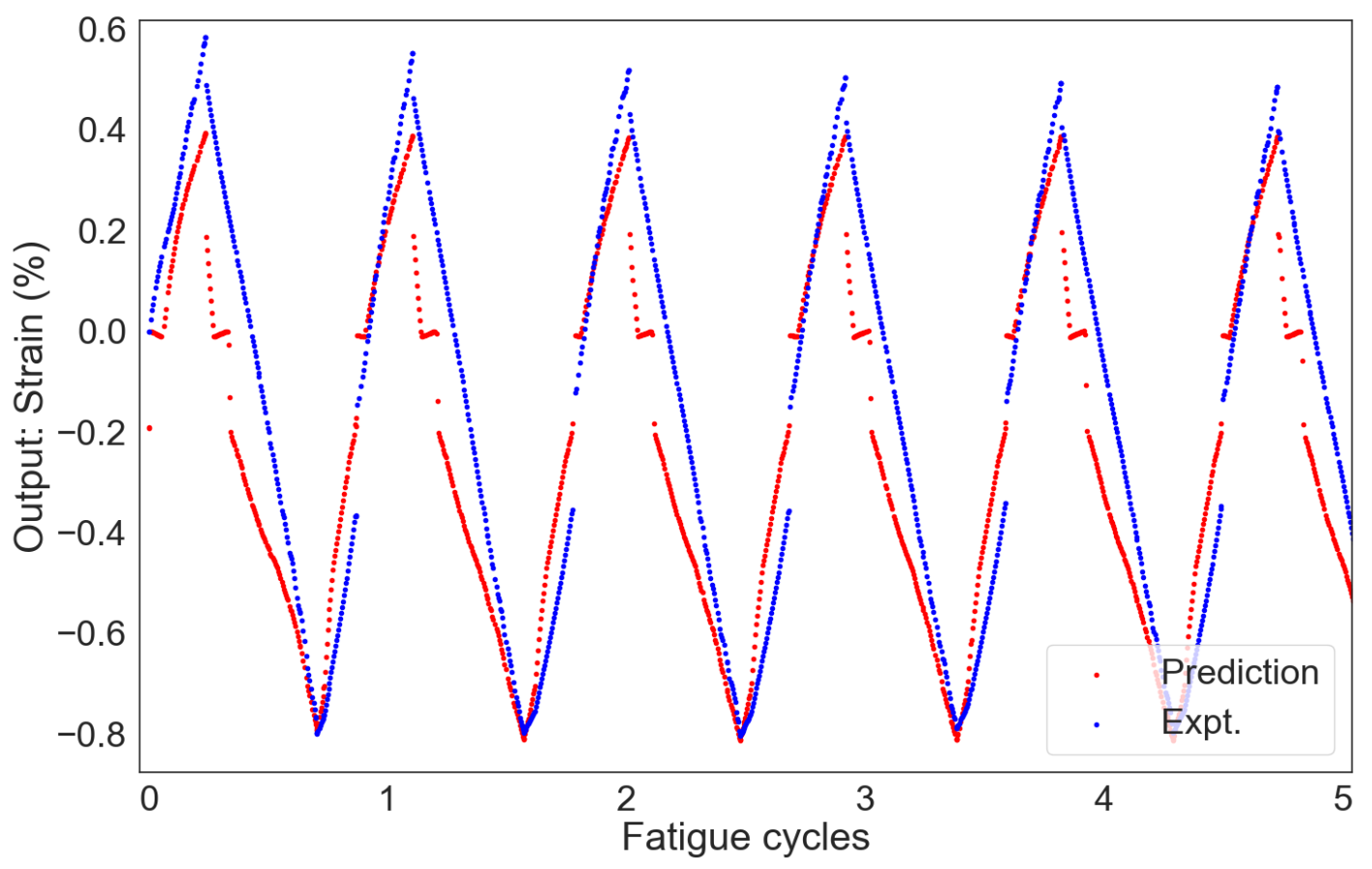

Figure 5.29 Cyclic strain estimation using the pull-rod displacement as the only independent variable. 


\subsubsection{Accuracy associated with different type of Al techniques under symmetrical cyclic loading}

Similar as unsymmetrical loading case, we tested the efficacy of different AI/ML/DL techniques for symmetrical loading test case: ET-F61. We considered first 50 cycles data for checking the model accuracy. The pool of 50 cycle data was randomly divided into training and test data with 70\%/30\% ratio. We conducted 100 randomized iterations (for randomly selecting training and test data with $70 \% / 30 \%$ ratio. We checked the efficacy of Scikit-learn based multi-dimensional-linear regression technique and Keras based multi-dimensional DL regression techniques. We checked the efficacy of both Sigmoid and ReLU activation function for Keras DL model. For all the model cases (used in this AI/ML/DL technique accuracy checking exercise), a single-step modeling approach were followed. Figures 5.30 to 5.33 show the Scikit-learn based model results. Whereas, Figures 5.34 to 5.37 and Figures 5.38 to 5.41 show the Kreas DL model results with respect to Sigmoid and ReLU activation function. Table 5.2 shows the overall comparison of CPU time and accuracy for 100 randomized iterations while predicting strain for the first 50 cycles of ET-F61 test case. From the table, similar as for unsymmetrical loading case the Keras DL model with ReLU activation function produced most accurate prediction of strain with maximum MSE of 0.0002. From Table 5.2, it can be seen that Scikit-learn model produce comparable accuracy compared to the Keras DL model. This is probably due to symmetric loading condition with lower nonstationary fatigue data compared to the unsymmetrical loading. Based on this results and judgement, we selected Keras DL model with ReLU activation function for further discussed strain-prediction results.

Table 5. 2 CPU time and accuracy for 100 randomized iterations while predicting strain for first 50 cycles of ET-F61 test case.

\begin{tabular}{|c|c|c|c|}
\hline $\begin{array}{c}\text { AI/ML/DL } \\
\text { Techniques }\end{array}$ & $\begin{array}{c}\text { Scikit-learn AI/ML } \\
\text { Library with multi- } \\
\text { dimensional linear } \\
\text { and single-step } \\
\text { regression }\end{array}$ & $\begin{array}{c}\text { Keras AI/DL library } \\
\text { with Sigmoid } \\
\text { activation function } \\
\text { and multi- } \\
\text { dimensional -single- } \\
\text { step regression }\end{array}$ & $\begin{array}{c}\text { Keras AI/DL library } \\
\text { with ReLU activation } \\
\text { function and multi- } \\
\text { dimensional -single- } \\
\text { step regression }\end{array}$ \\
\hline $\begin{array}{c}\text { CPU Time }(s) \\
\text { Accuracy: Highest } \\
\text { MSE observed }\end{array}$ & 0.00031 & 0.023 .32 & 6651.8 \\
\hline
\end{tabular}


5.2.2.1 Cyclic strain estimation using AI/DL based Scikit-learn library

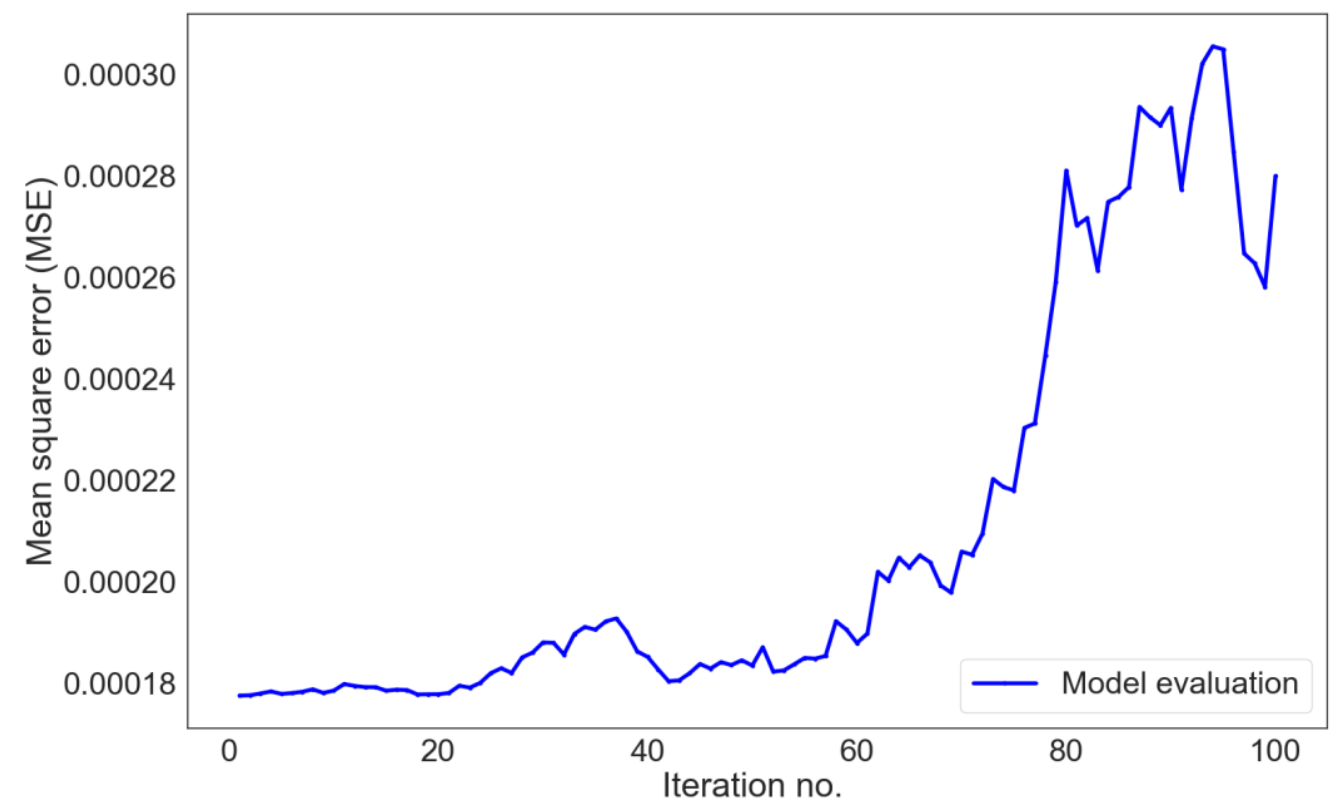

Figure 5. 30 Scikit-learn library-based MSE (with respect to different randomized sampling iterations), while predicting strain for first 50 cycles of ET-F61 test case.

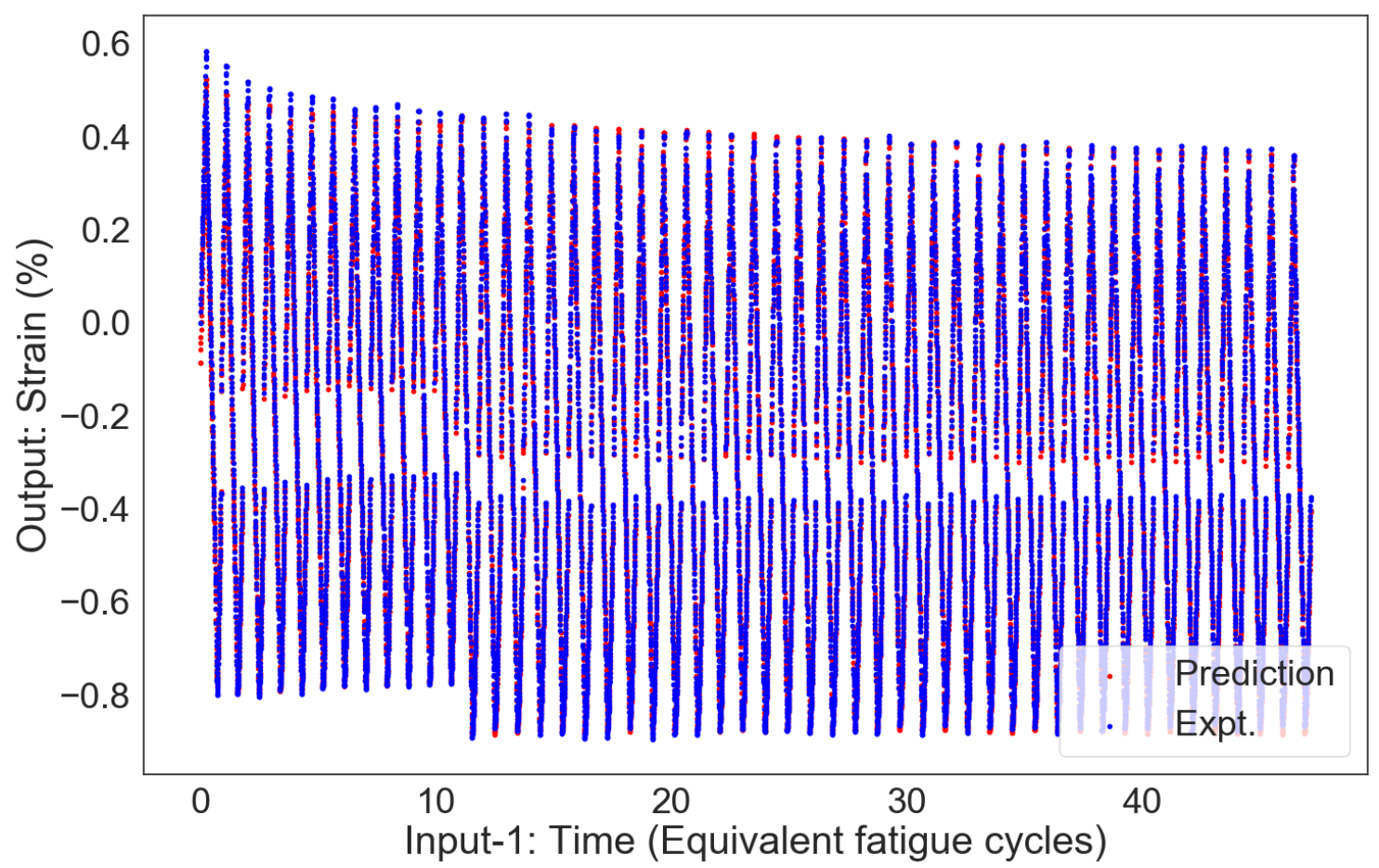

Figure 5. 31 Scikit-learn library-based predicted strain (for first 50 cycles of ET-F61 test case) and its comparison to corresponding experimental strain. 


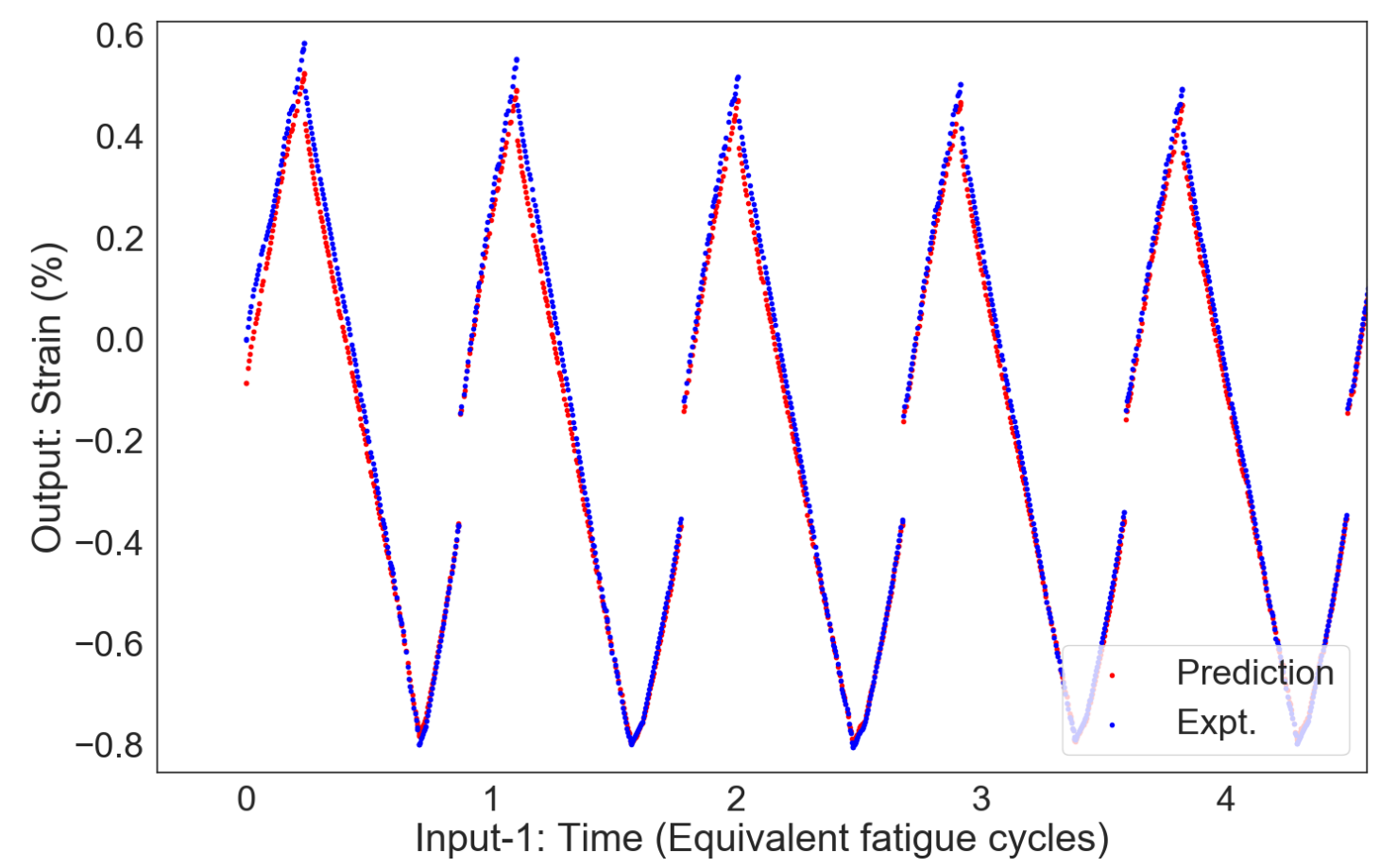

Figure 5. 32 Magnified version of Figure 5.31.

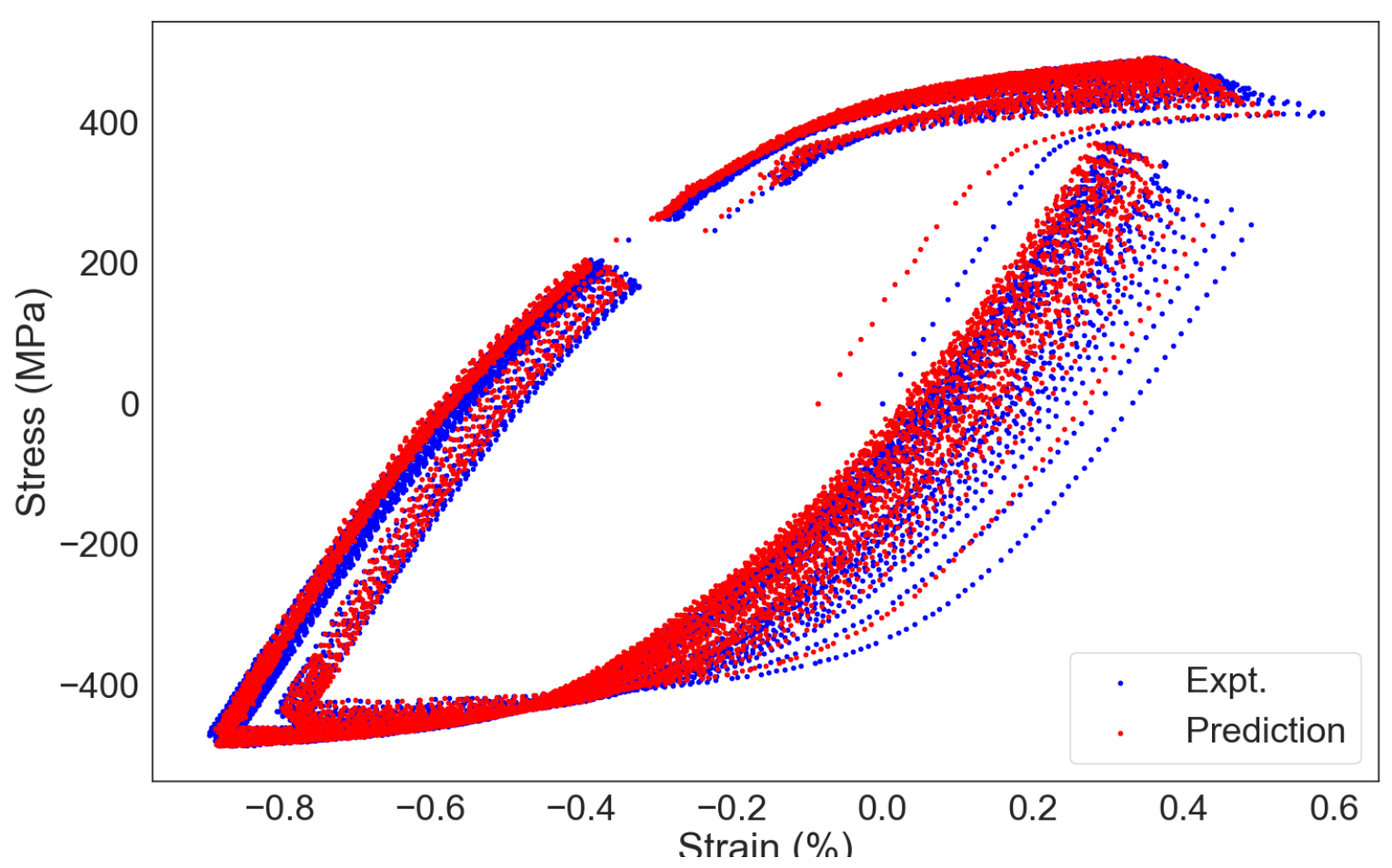

Figure 5. 33 Scikit-learn library-based predicted strain hysteresis curves (for first 50 cycles of ET-F61 test case) and its comparison to corresponding experimental strain hysteresis curves. 
5.2.2.2 Cyclic strain estimation using AI/DL based Keras library and Sigmoid activation function

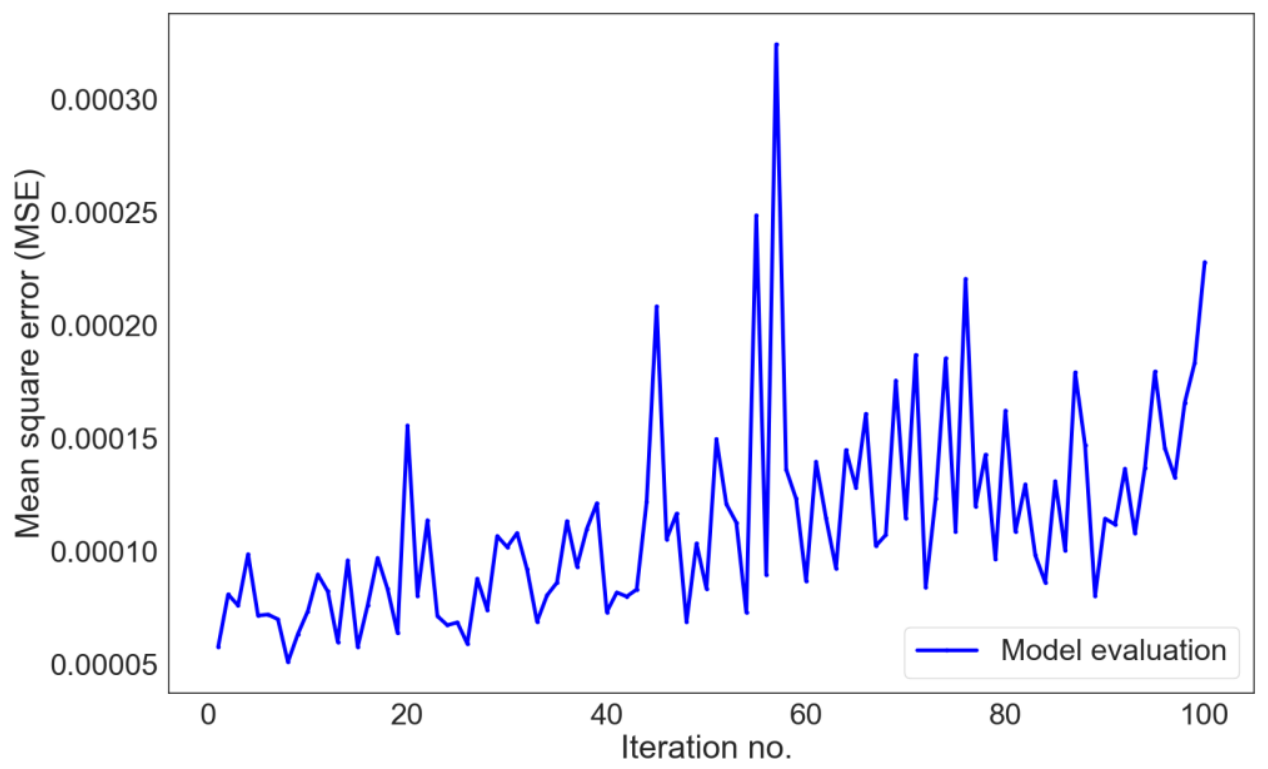

Figure 5.34 Keras library and Sigmoid activation-function based MSE (with respect to different randomized sampling iterations), while predicting strain for first 50 cycles of ET-F61 test case.

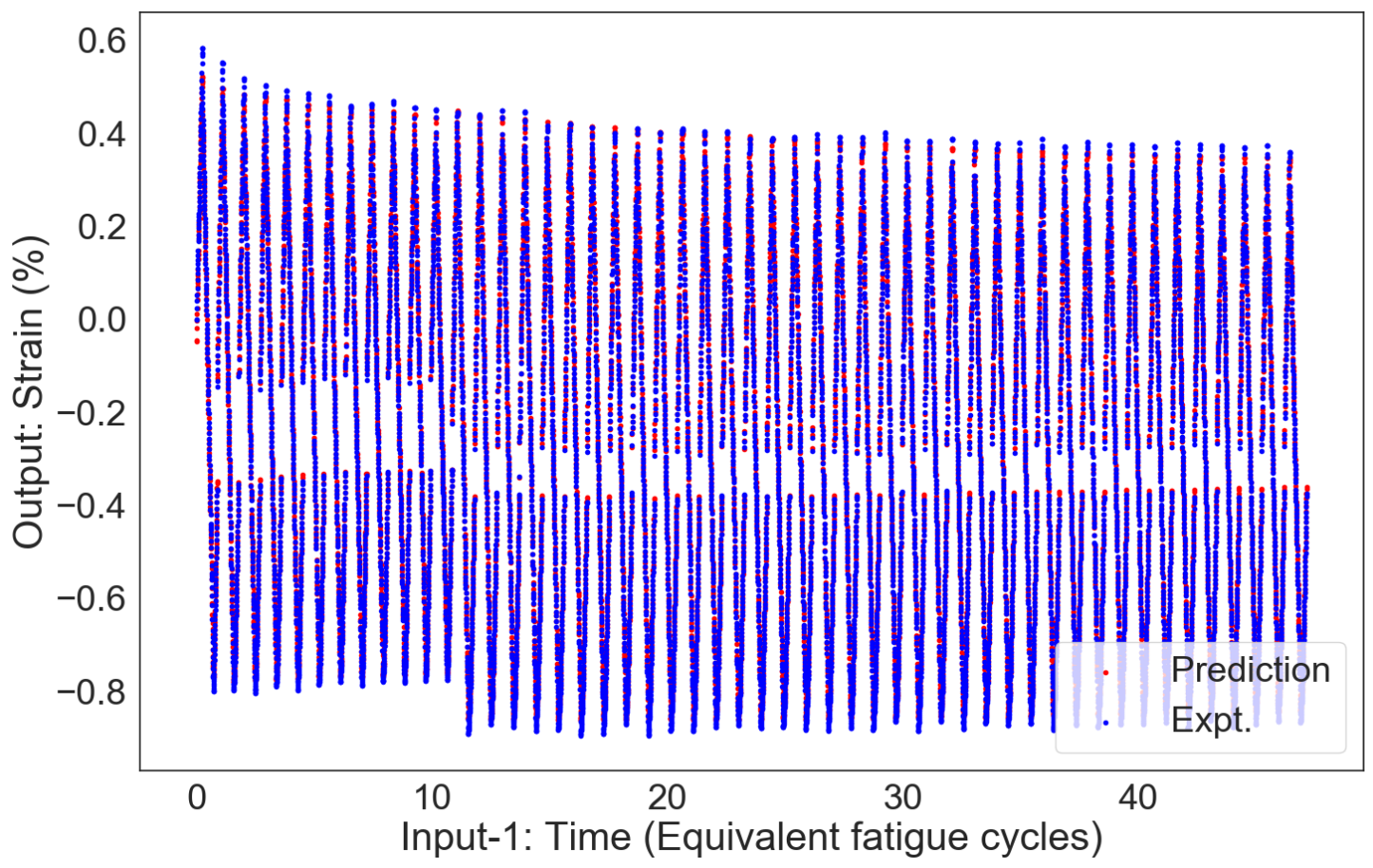

Figure 5. 35 Keras library and Sigmoid activation-function based predicted strain (for first 50 cycles of ET-F61 test case) and its comparison to corresponding experimental strain. 


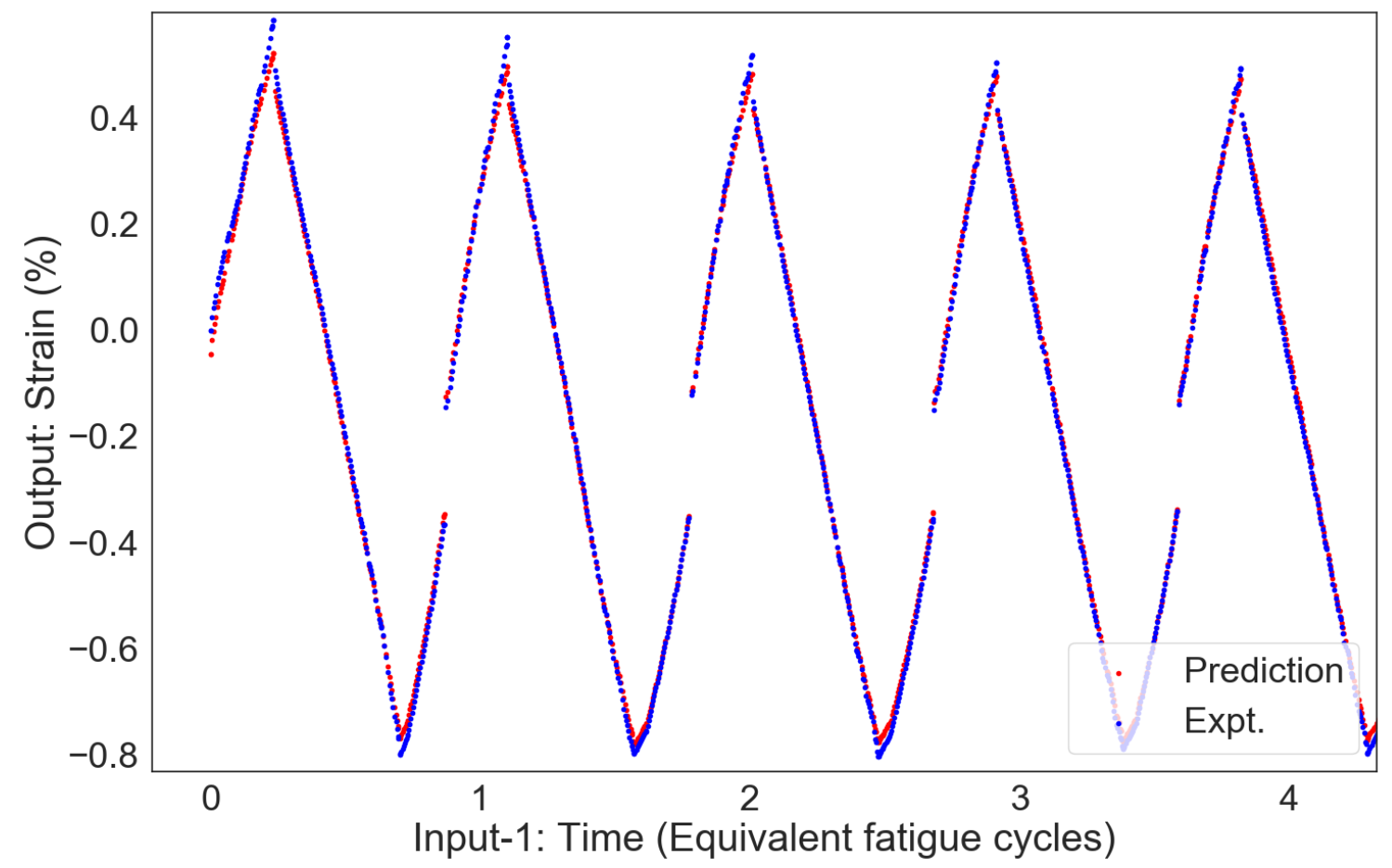

Figure 5. 36 Magnified version of Figure 5.35.

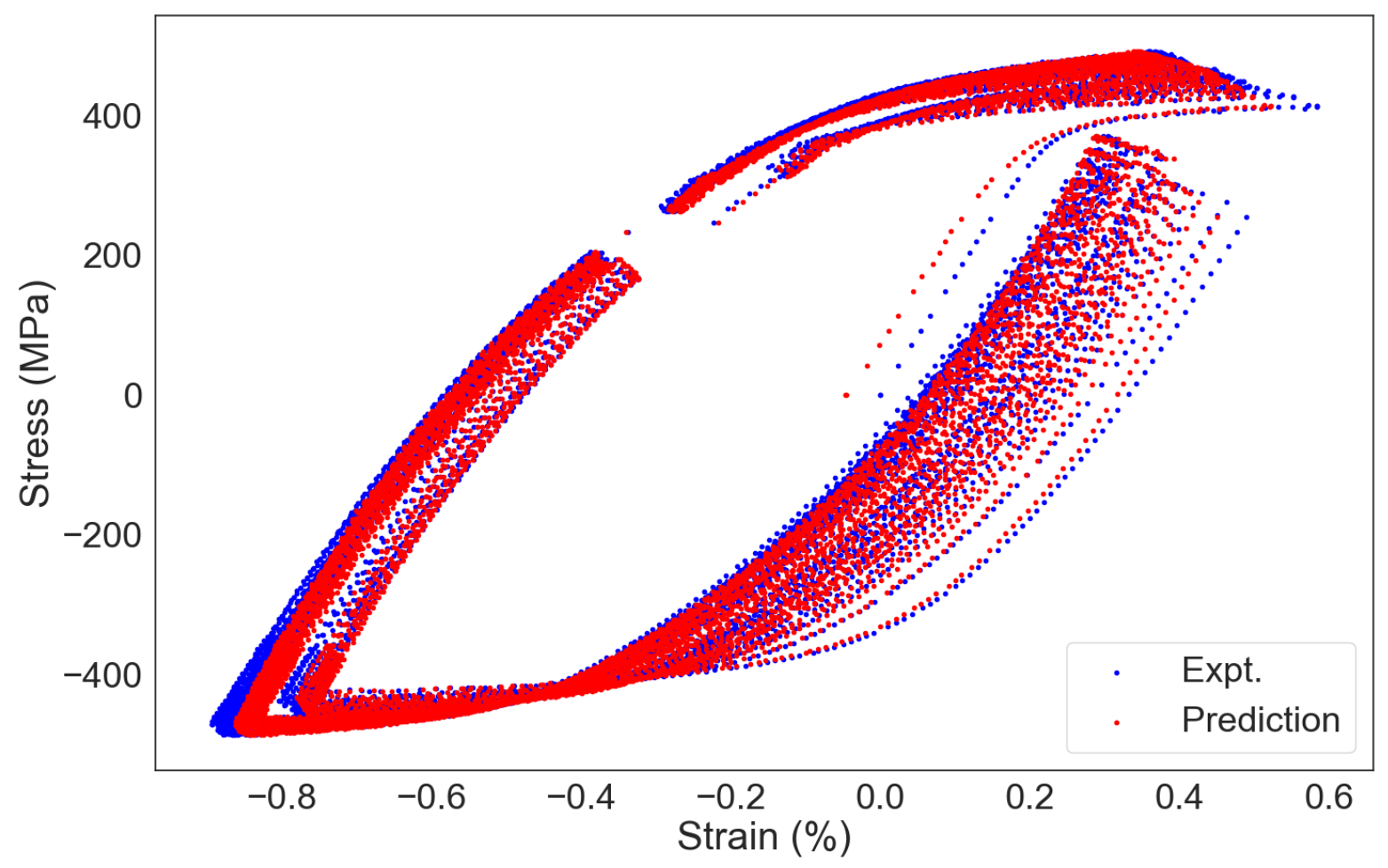

Figure 5. 37 Keras library and Sigmoid activation-function based hysteresis curves (for first 50 cycles of ET-F61 test case) and its comparison to corresponding experimental strain hysteresis curves. 
5.2.2.3 Cyclic strain estimation using AI/DL based Keras library and ReLU activation function

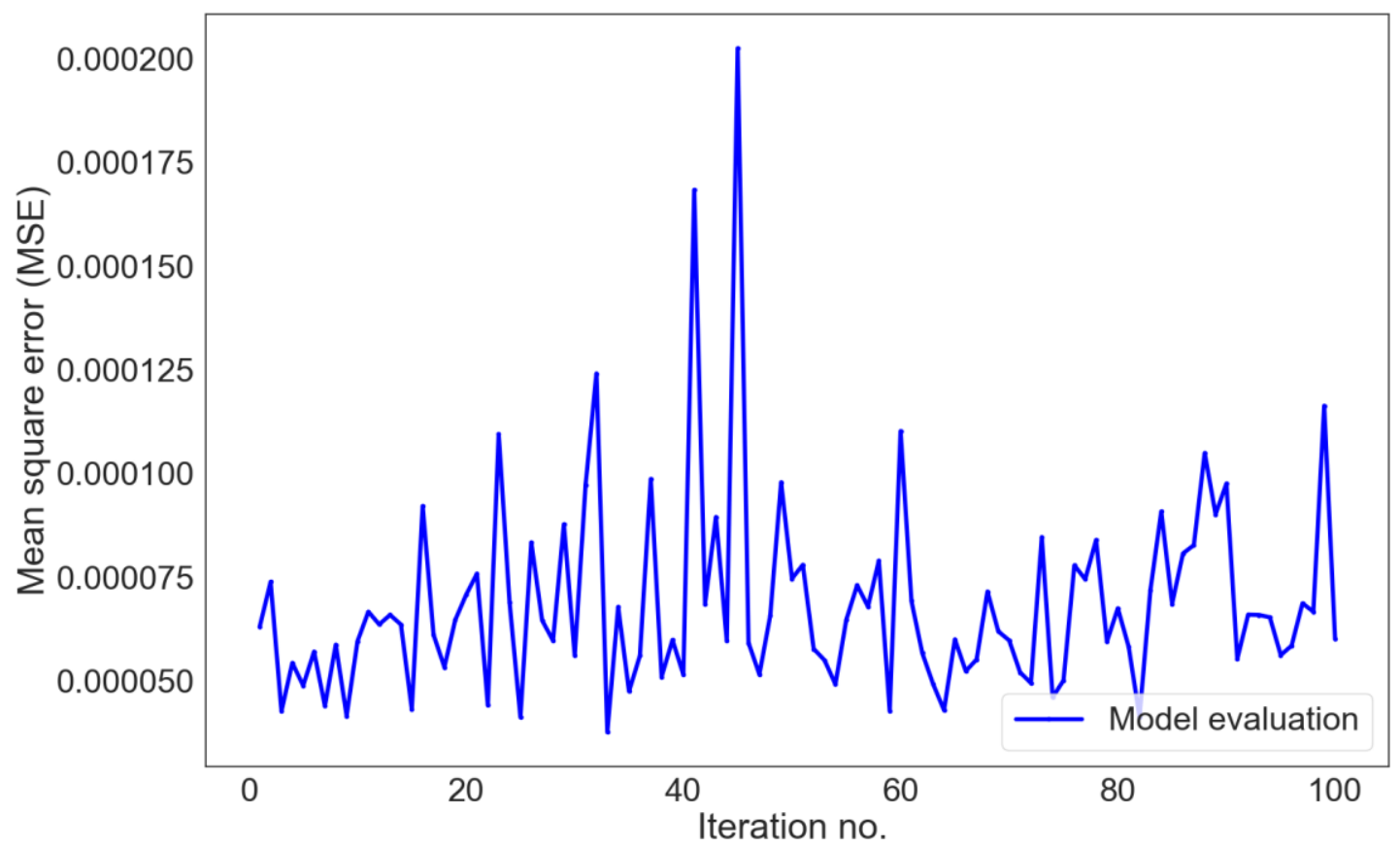

Figure 5. 38 Keras library and ReLU activation-function based MSE (with respect to different randomized sampling iterations), while predicting strain for first 50 cycles of ET-F61 test case.

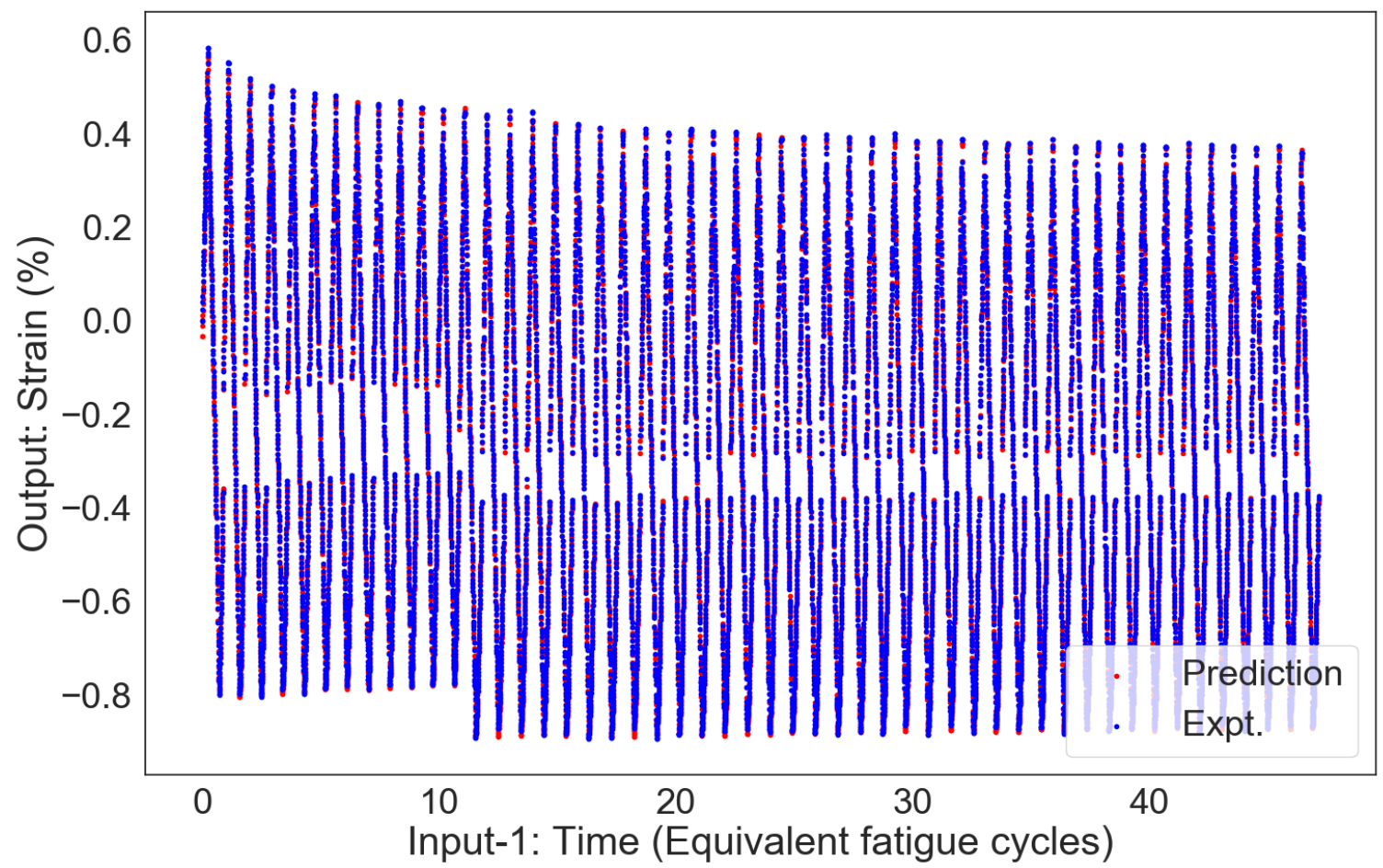

Figure 5. 39 Keras library and ReLU activation-function based predicted strain (for first 50 cycles of ETF61 test case) and its comparison to corresponding experimental strain. 


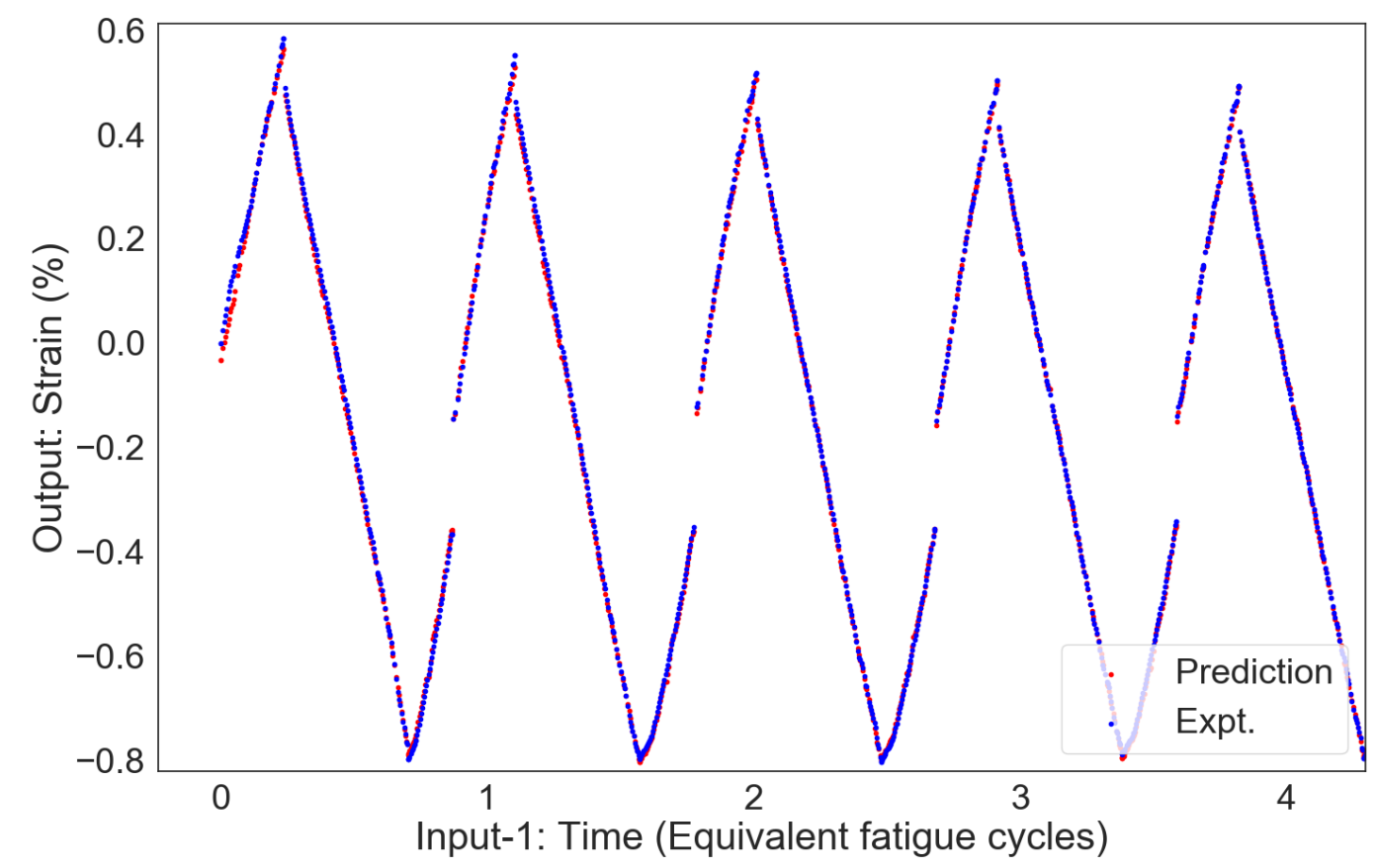

Figure 5. 40 Magnified version of Figure 5.39.

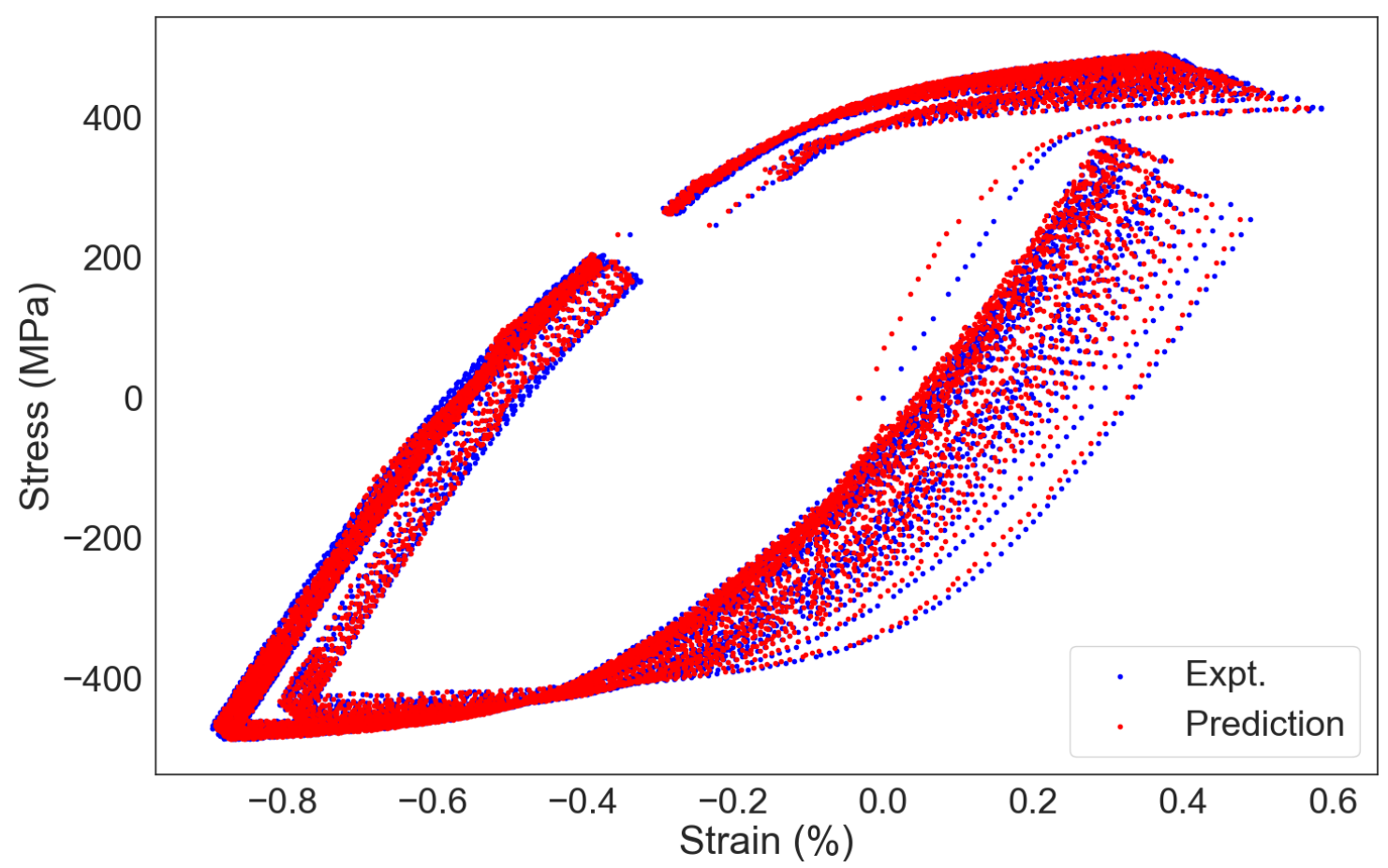

Figure 5. 41 Keras library and ReLU activation-function based hysteresis curves (for first 50 cycles of ET-F61 test case) and its comparison to corresponding experimental strain hysteresis curves. 


\subsubsection{Clustering and piecewise strain time-series estimation to deal with non-stationarity associated} with cyclic hardening under symmetrical cyclic loading

Similar to the unsymmetrical loading case, we used piecewise modeling to reduce the issue associated with cyclic hardening/softening and related non-stationarity in fatigue test data. The issue of non-stationarity is more prevalence, while dealing with fatigue data for entire fatigue cycles. Nonstationarity in ET-F61 fatigue test data can be found from the Figure 5.42 and 5.43. To deal with the non-stationarity we grouped/clustered the entire ET-F61 fatigue data to multiple subdomains. This is through using Scikit-learn based k-mean clustering technique (refer to section 2.6). Figures 5.44 and 5.45 show the cluster analysis related results. Based on the clustered data set and Keras DL library and ReLU activation function, different set of model parameters were estimated to model the entire fatigue life of ET-F61 specimen. Figures 5.46 to 5.50 shows the related prediction versus experiment results. These results show that very accurate prediction of the strain. This is by not only capturing its time/cycle dependency but also capturing the shape of the highly nonlinear hysteresis loop. With this confidence, we further used the Keras DL library and ReLU activation function-based predictive model for strain estimation under inaccessible PWR-loop autoclave in the later part of this report.

\subsubsection{Non-stationarity process associated with cyclic hardening}

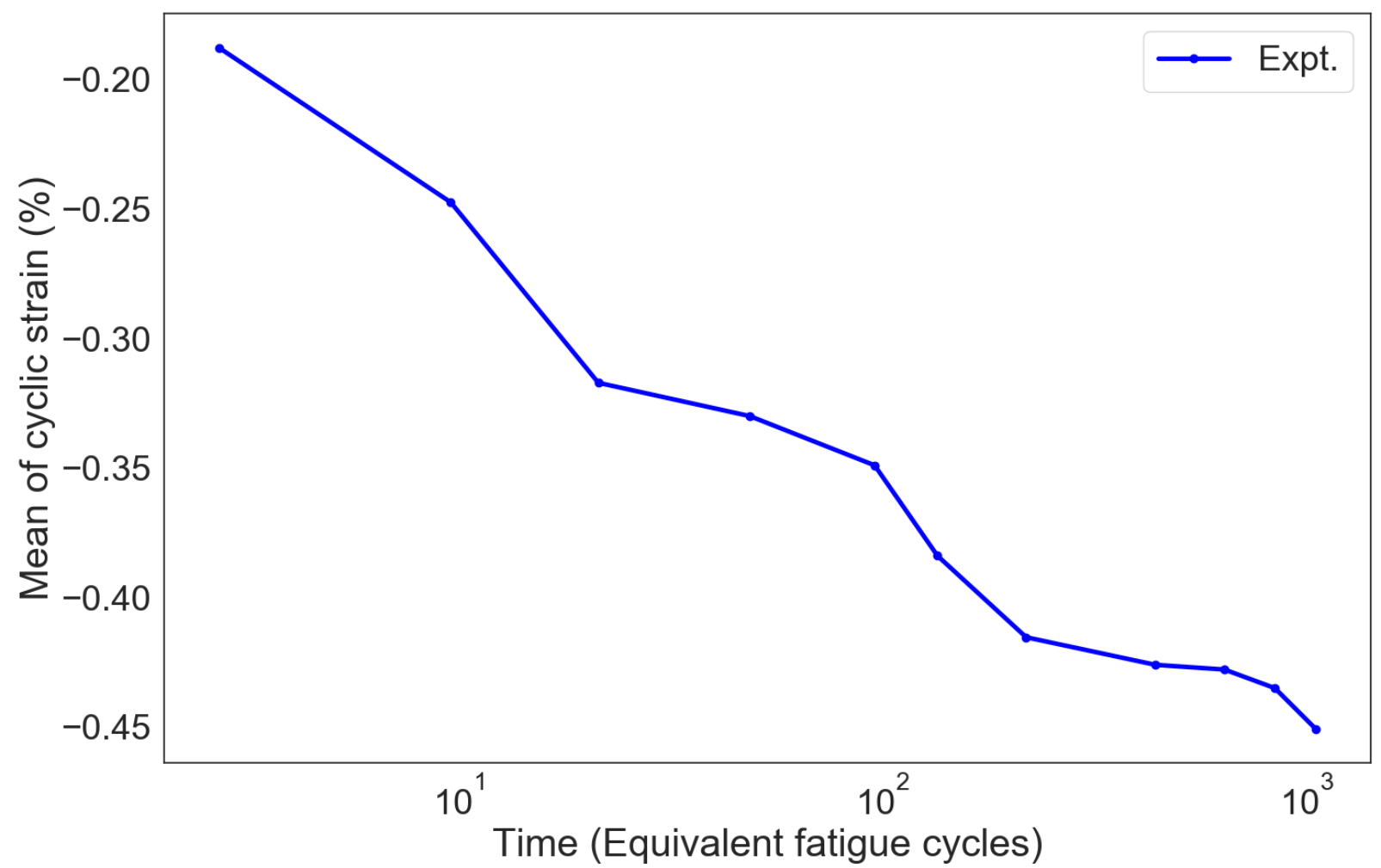

Figure 5. 42 Substantial variation of mean of ET-F61 cyclic strains shows that the time-series strain for the entire fatigue life follows a non-stationary process. 


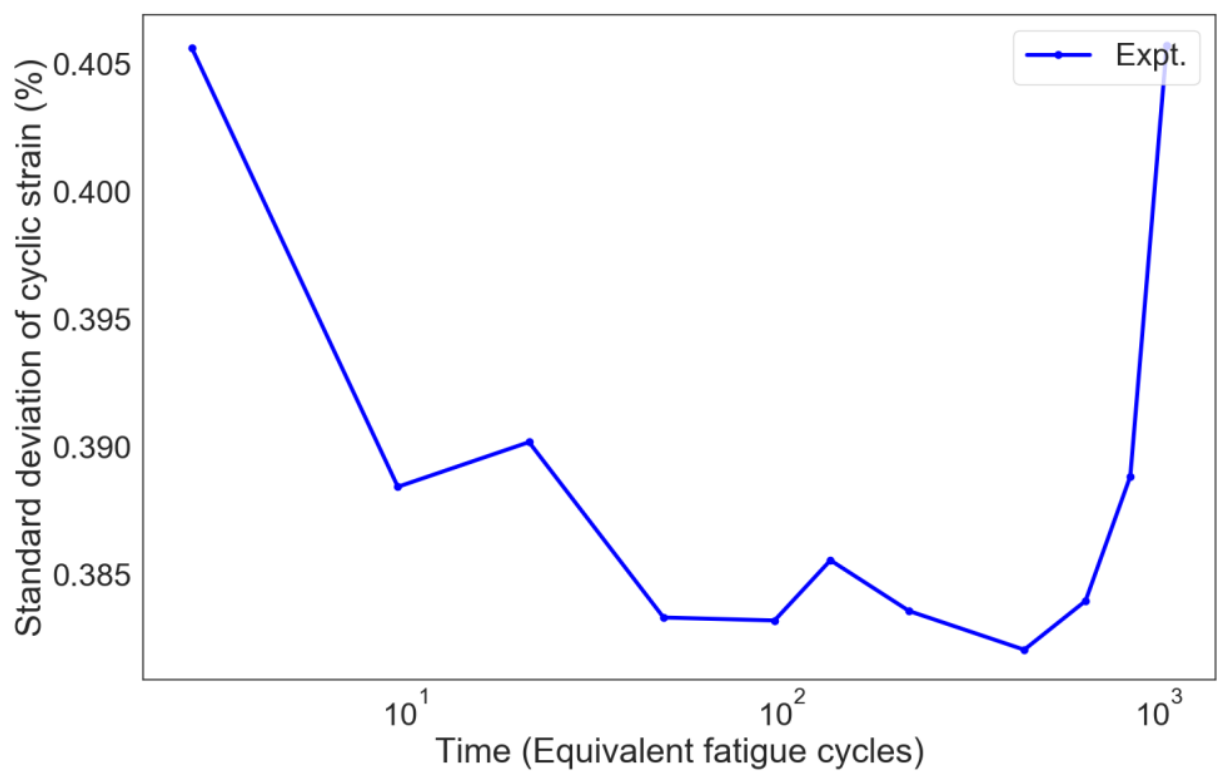

Figure 5. 43 Variation of standard deviation of ET-F61 cyclic strains shows that the time-series strain for the entire fatigue life follows a non-stationary process.

5.2.3.2 Scikit-learn based K-Mean clustering to divide the entire fatigue life to multiple subdomains

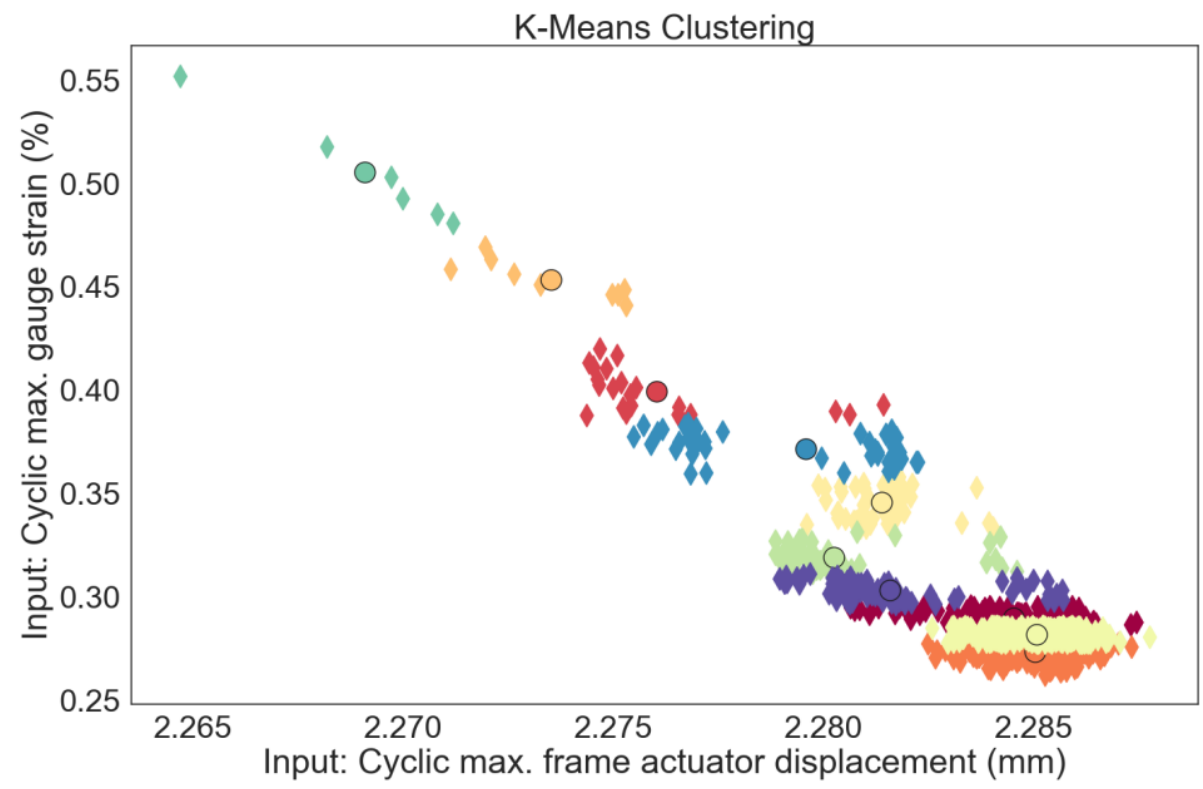

Figure 5. 44 ET-F61 clustered data showing the 2-dimensional dependency between frame maximum actuator displacement and corresponding specimen gage area maximum strain. Raw data in an individual cluster (plotted with a single color) are reparented by dots whereas, the solid circles represent the corresponding cluster center. 


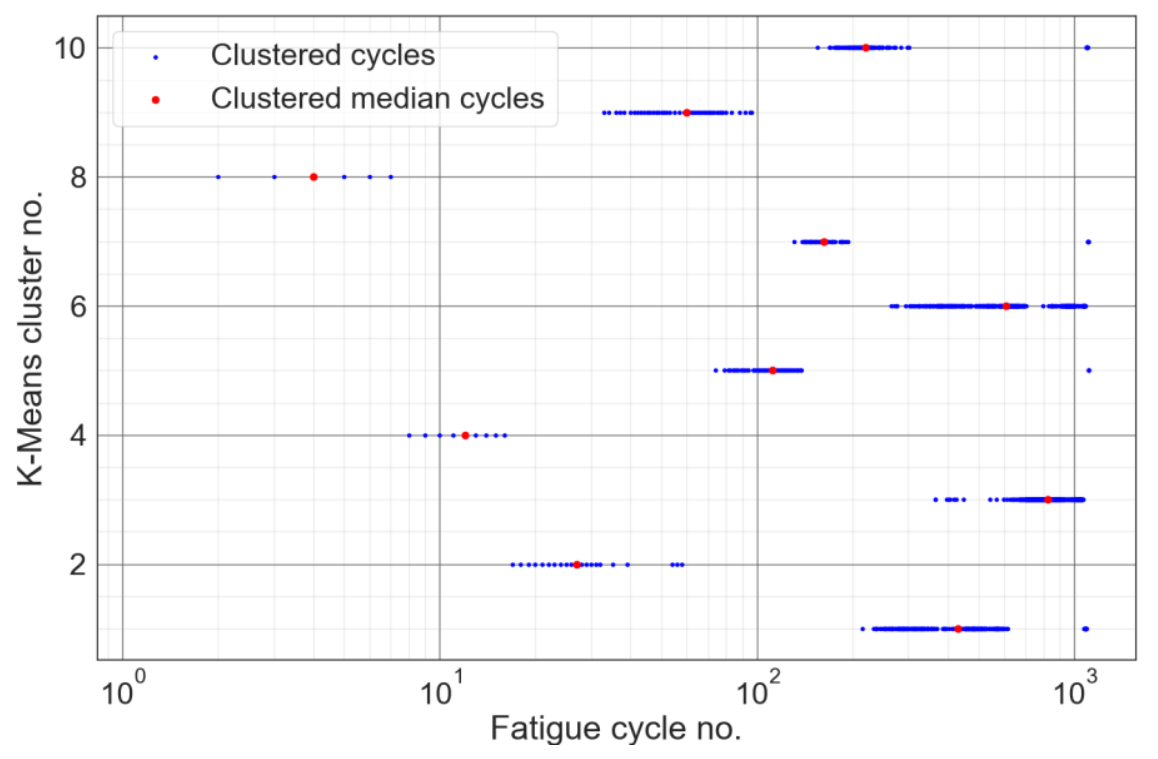

Figure 5. 45 Cycle versus corresponding cluster numbers showing how the entire ET-F61 fatigue-test data clustered into only 10 different groups/clusters. Piecewise strain prediction models were developed for individual clusters to predict the strain for the entire fatigue life of ET-F61 test case.

5.2.3.3 Piecewise strain prediction for the entire fatigue life of ET-F61 specimen using AI/DL based Keras library and ReLU activation function

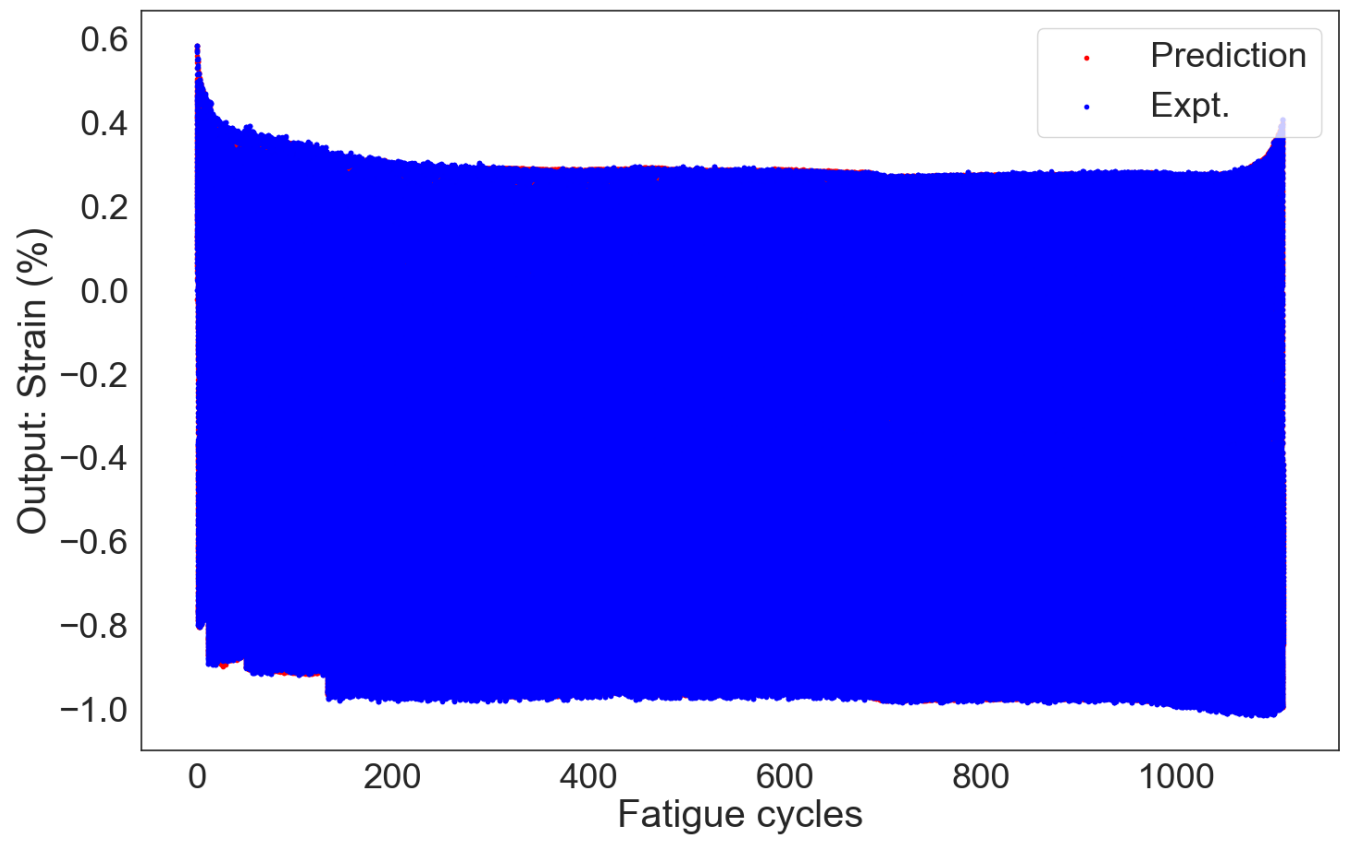

Figure 5. 46 Keras library and ReLU activation-function based predicted time-series strain (for the entire fatigue cycles of ET-F61 test case) and its comparison to corresponding experimental strain. 


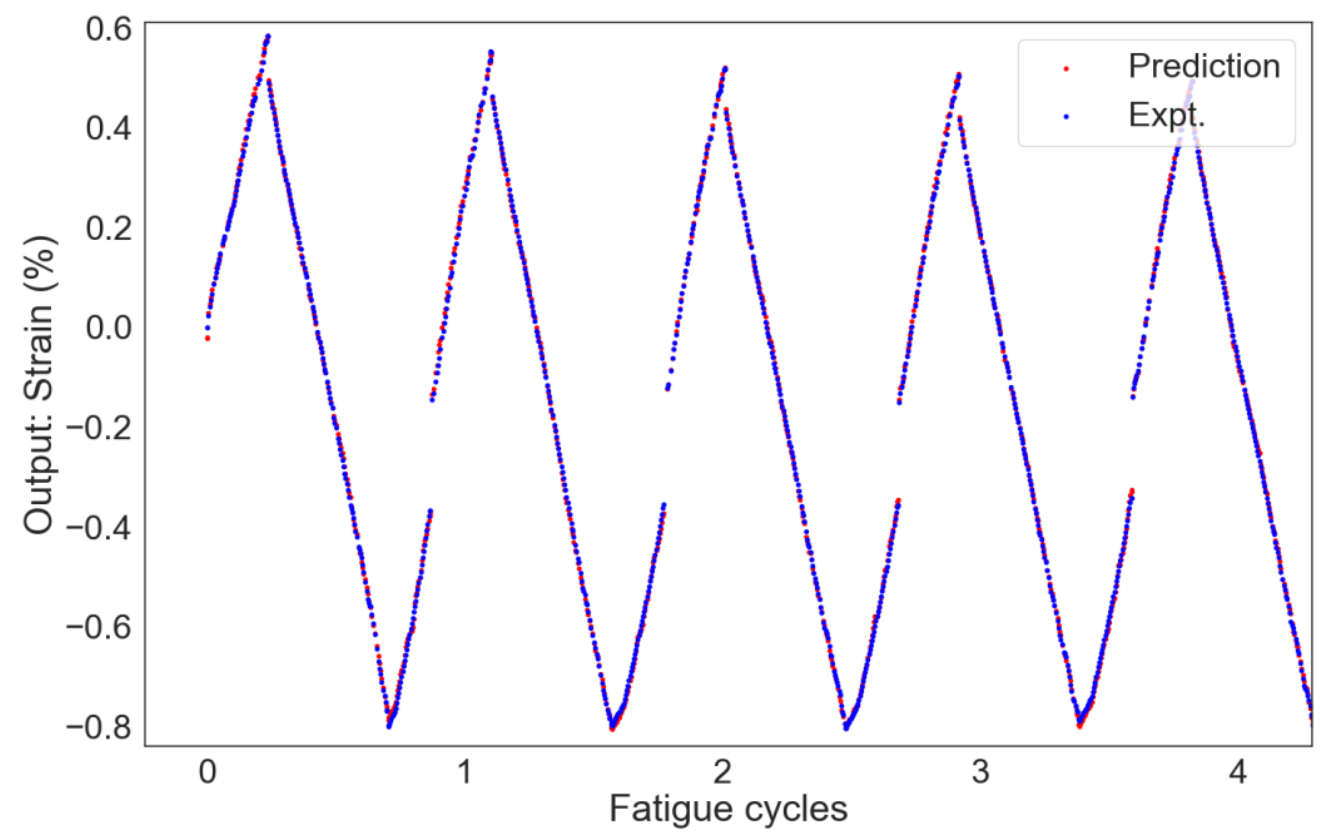

Figure 5. 47 Magnified version of Figure 5.46 showing first few cycles (of ET-F61 test case) prediction versus experimental results.

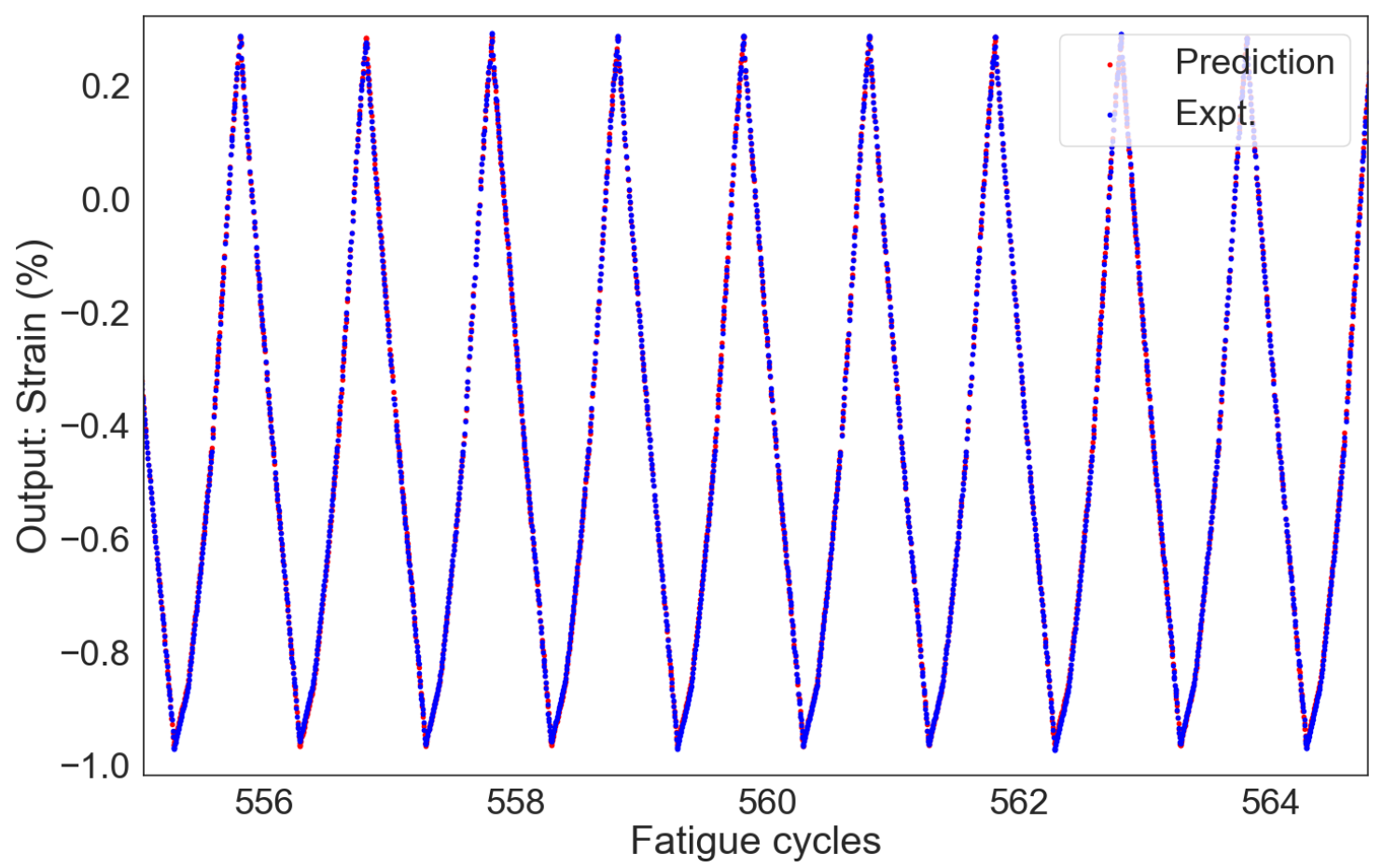

Figure 5. 48 Magnified version of Figure 5.46 showing few half-life cycles (of ET-F61 test case) prediction versus experimental results. 


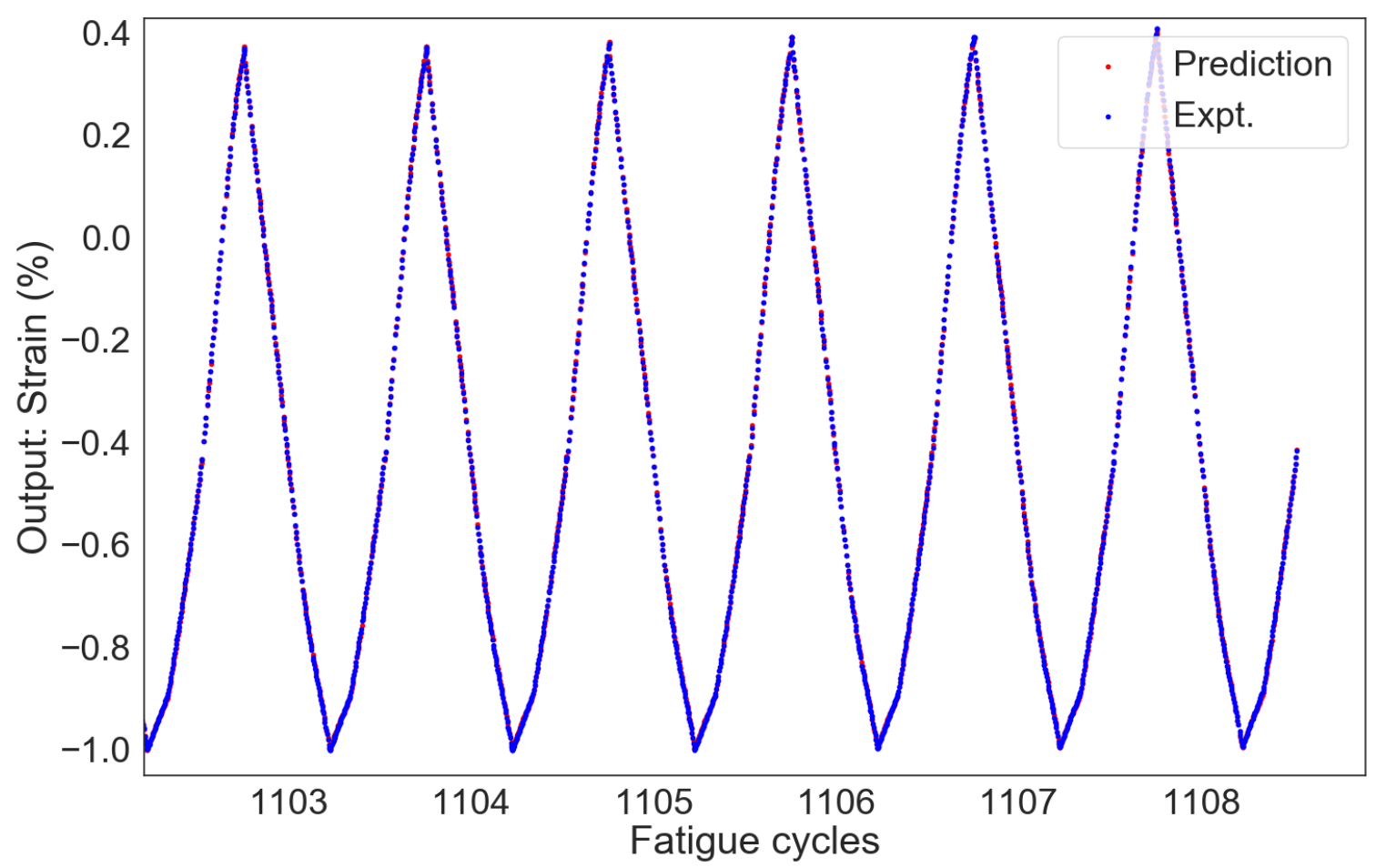

Figure 5. 49 Magnified version of Figure 5.46 showing few end-life cycles (of ET-F61 test case) prediction versus experimental results.

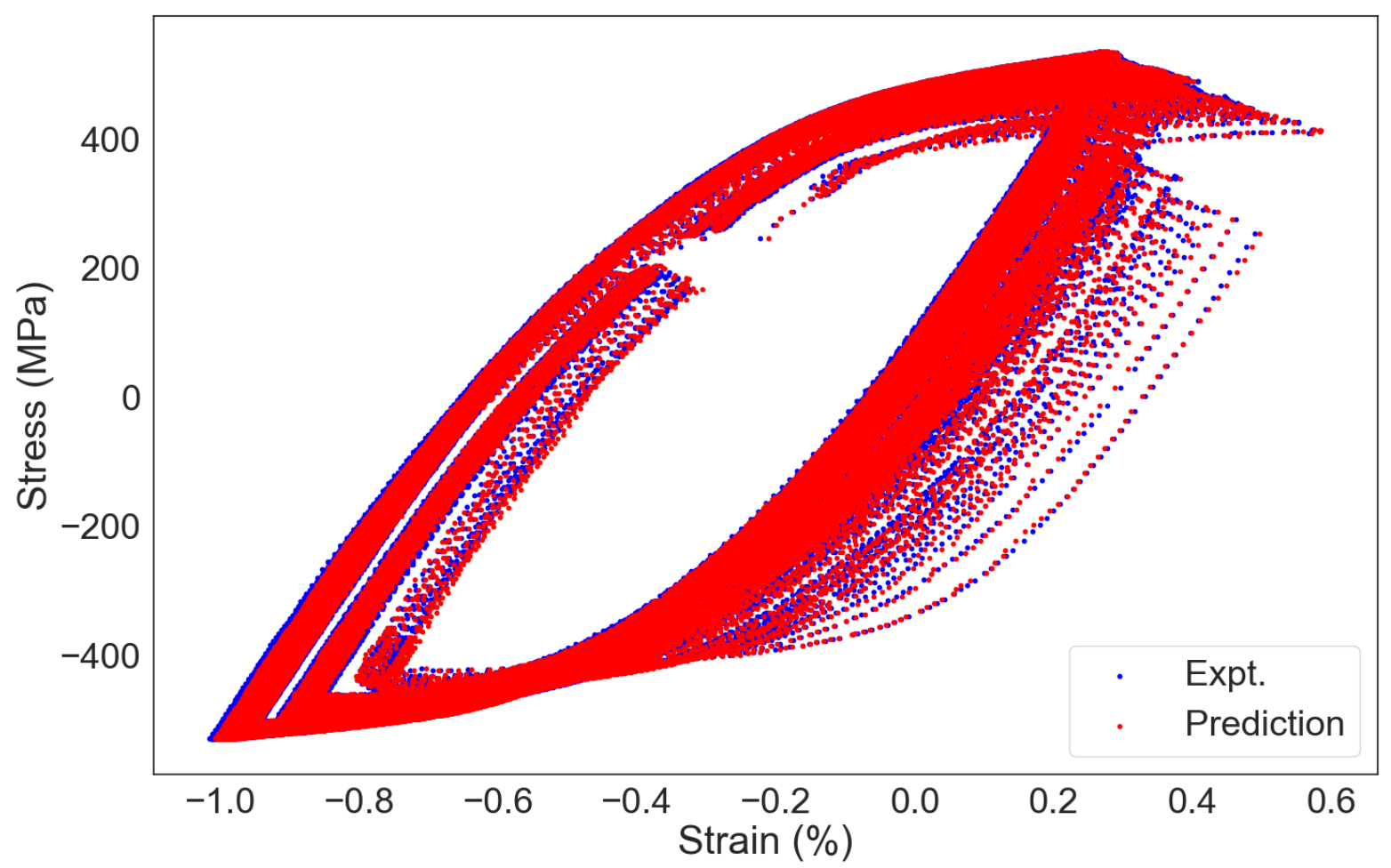

Figure 5. 50 Predicted strain hysteresis curves (for entire fatigue life of ET-F61 test case) and its comparison to corresponding experimental strain hysteresis curves. 


\subsection{Prediction Case: Cyclic Strain Prediction for Fatigue Test Specimen Subjected to EN-F62 Symmetrical Cyclic Loading and under PWR-Coolant-Water Environment}

Based on the above discussed two validation cases we found Keras DL library along with ReLU activation function produce most accurate prediction results. We used the ET-F61 in-air test data (which includes all the four input dimension: time, pull-rod-displacement measurements, frame-actuatordisplacement measurements and frame-load-cell measurements and the output/target: gauge strain measurements) and the Keras DL library along with ReLU activation function to estimate the model parameters. The associated model later used for predicting the cyclic strain of EN-F62 fatigue test case. Note that for a PWR-water-loop test, strain measurements are not available since an extensometer could not be used in a PWR-water test loop. So, with this difficulty of direct strain measurements in a PWRwater test loop, we can make use of the above discussed AI/ML/DL based models to predict the cyclic strains based on the other sensor measurements. We used the known input dimension data (time, pullrod-displacement measurements, frame-actuator-displacement measurements and frame-load-cell measurements) of EN-F62 to predict the corresponding unknown/unmeasurable cyclic strains. Based on the above discussed extensive validation results, we assume the predicted cyclic strain not only accurately capture its time-dependency (associated with cyclic hardening/softening), but also captures the shape of highly nonlinear hysteresis behavior (associated with path dependency under fatigue loading). Figures 5.51 to 5.55 show the related prediction results. Note that in Figures 5.51 to 5.55 it can be seen that the experimental strain is zero since there was no strain measurements available for PWRwater-loop test. However, that doesn't mean the specimen was not experiencing any strain. In addition, from Figures 5.51 to 5.55, it can be seen that the predicted strains are not symmetric about zero. While developing the material models, the strain-axis need to be shifted by removing the respective mean strain.

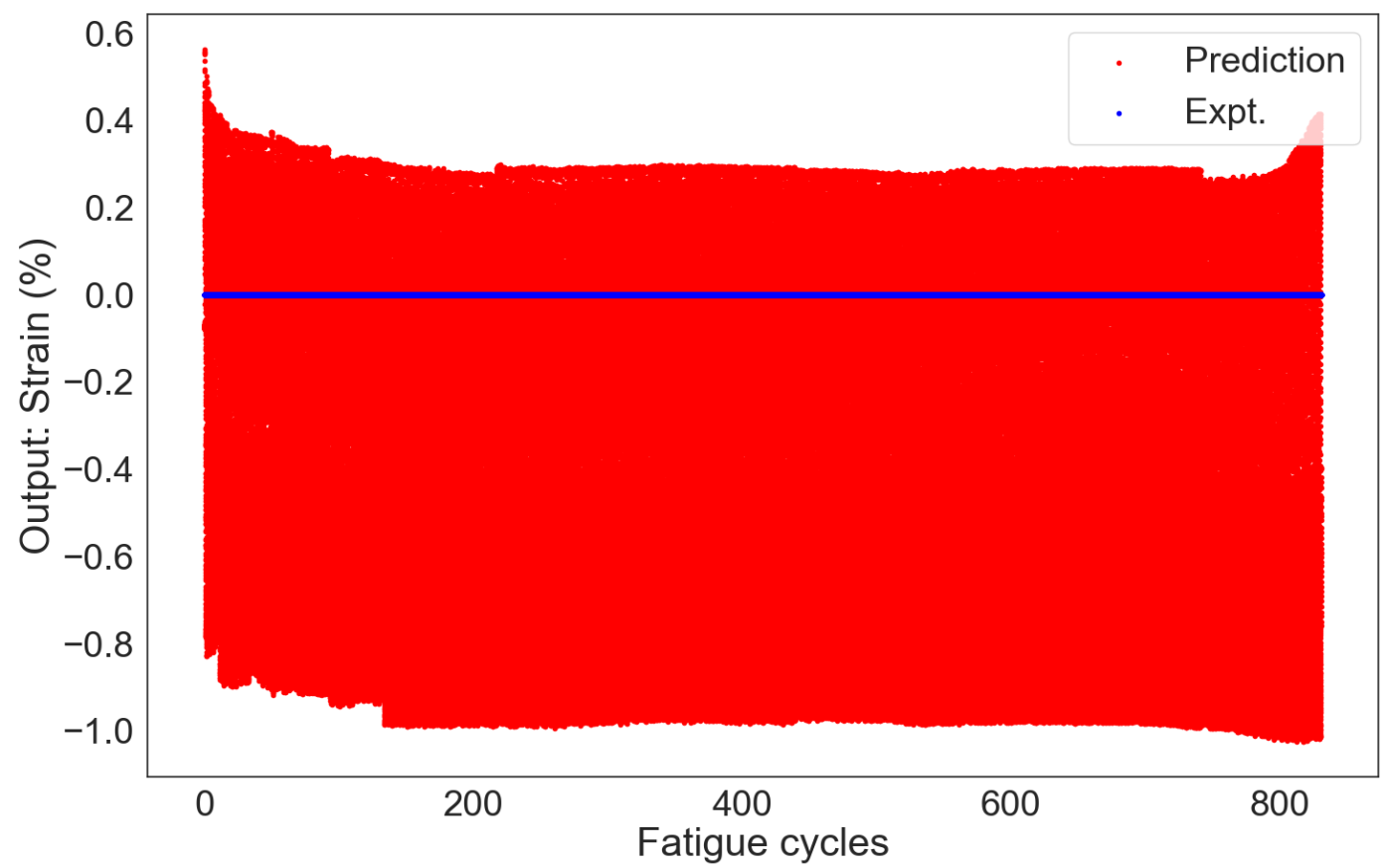

Figure 5. 51 Keras library and ReLU activation-function based predicted time-series strain for the entire fatigue cycles of EN-F62 test case. 


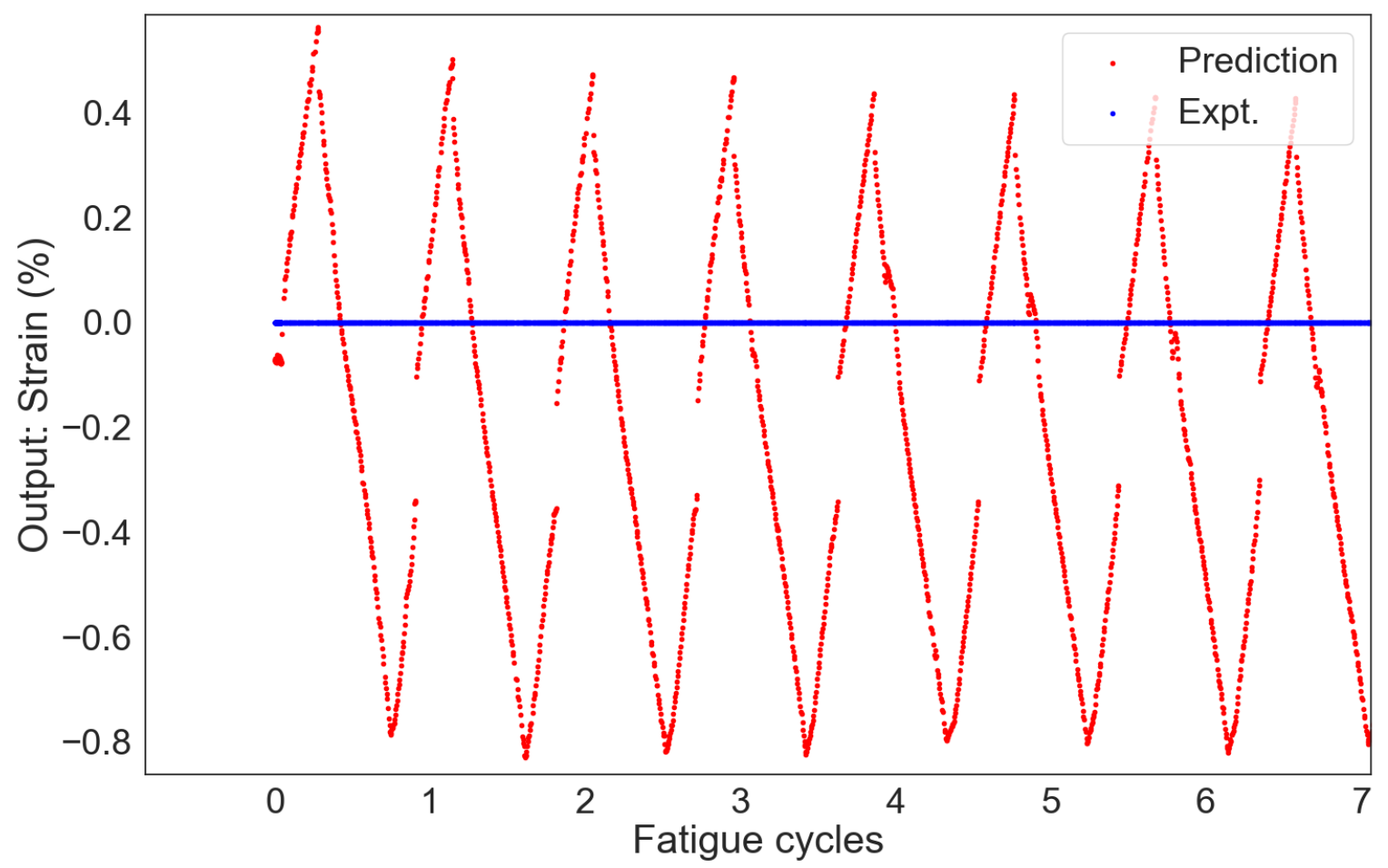

Figure 5. 52 Magnified version of Figure 5.51 showing strain prediction results for first few cycles of EN-F62 test case.

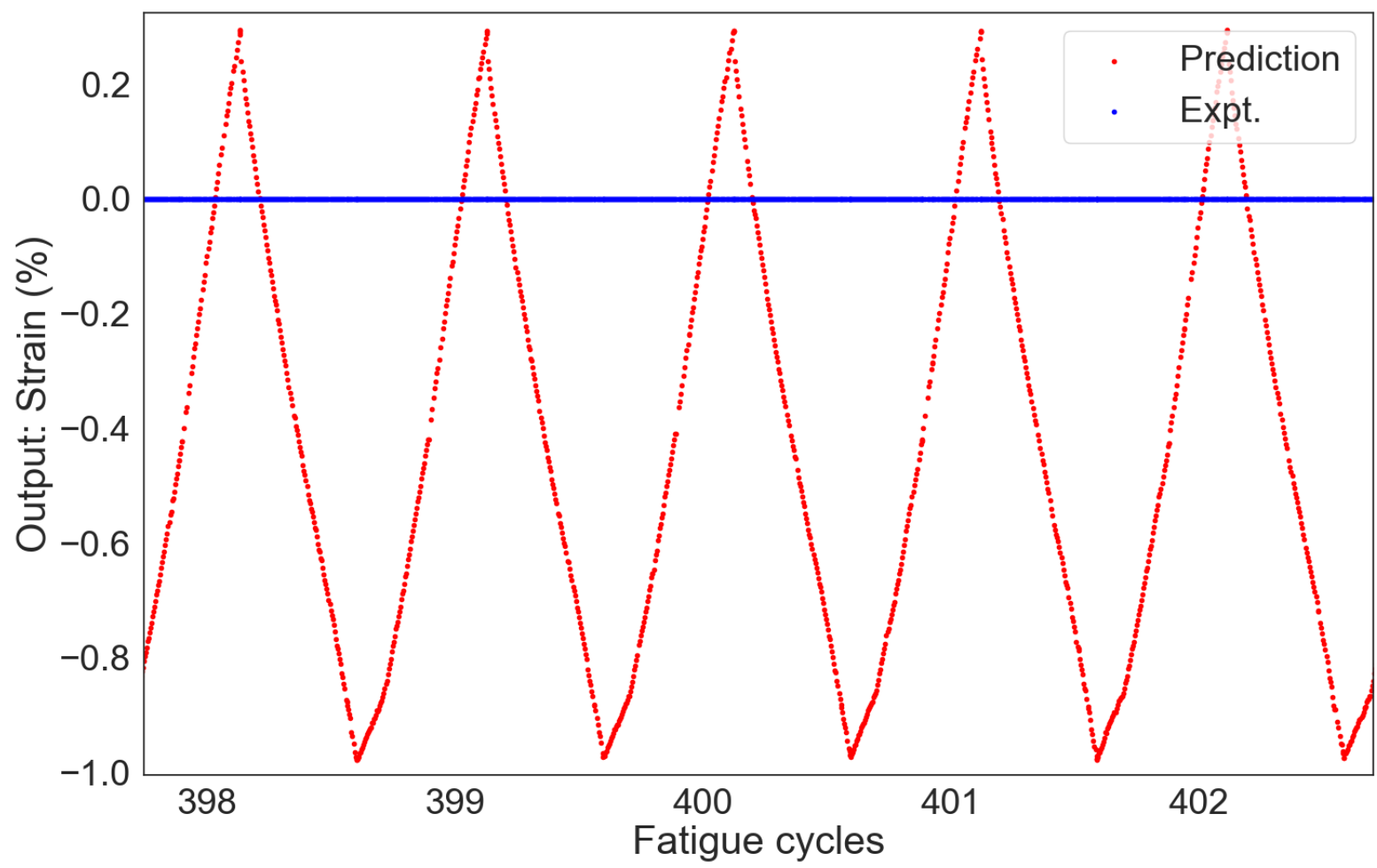

Figure 5. 53 Magnified version of 5.51 showing strain prediction results for few half-life cycles of ENF62 test case. 


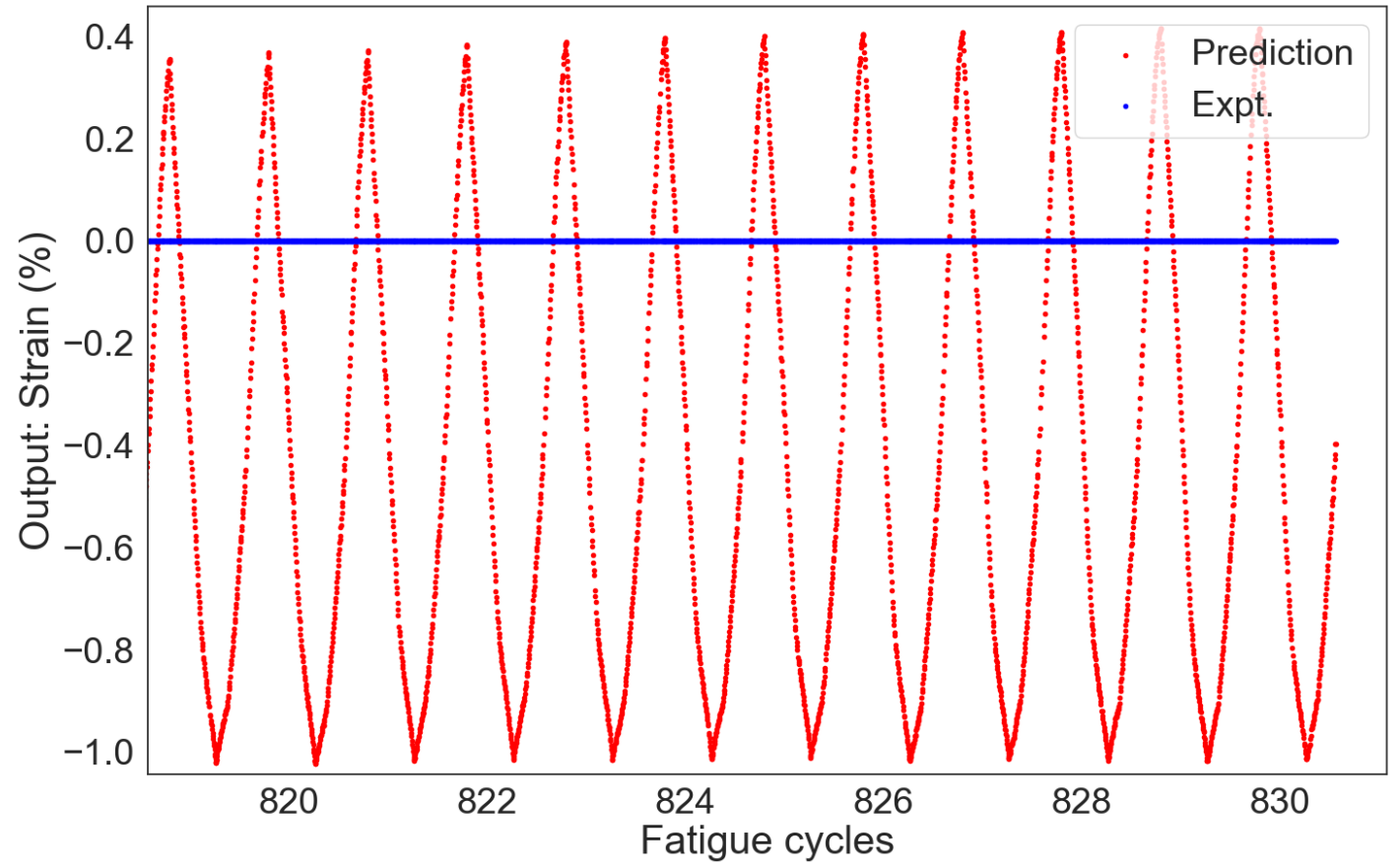

Figure 5. 54 Magnified version of 5.51 showing strain prediction results for few end-life cycles of ENF62 test case.

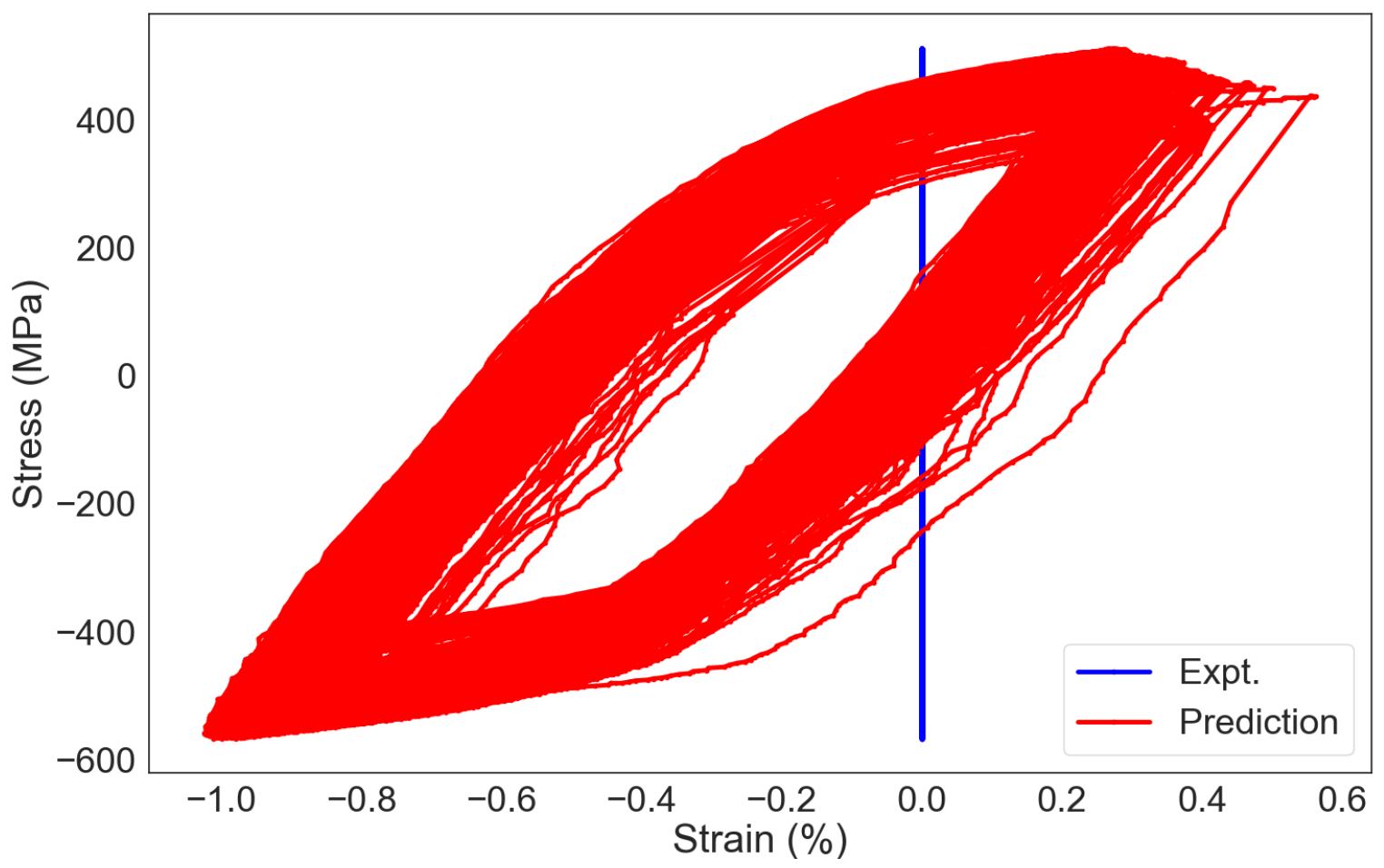

Figure 5. 55 Predicted strain hysteresis curves for entire fatigue life of EN-F62 test case. 


\section{A Hybrid Al-TensorFlow and Computational-Mechanics Based Approach for Cyclic Material Parameter Prediction}

In this section, we present the results related to AI/ML based technique such as using TensorFlow to estimate the cyclic stress strain curves from the cyclic strain versus stress data. Later we used the estimated cyclic stress-strain curves and computational-mechanics based constitutive models to predict cyclic material model parameters. Note that in previous work [17], we presented the material model parameters for DMW, based on the tensile test data. Although the tensile test-based data can be used for firsthand FE modeling of reactor components, these data might not capture the cyclic hardening/softening of reactor component subjected to cyclic loading. In this FY we tried to develop cyclic plasticity material models of DMW, in an anticipation that resulting material model parameters can help nuclear reactor industry and regulators for predicting the component stress more accurately. However, cycle material model parameters need to be estimated from cyclic stress-strain curves. The cyclic stress-strain curves can be estimated based on combined use of stress-strain data obtained from different strain-controlled fatigue tests with different strain amplitudes. However, this approach requires multiple fatigue tests to be conducted, which is expensive and time consuming. Rather, we conducted a single variable amplitude test (ET-F57), comprising of repeating block loading with each block having 12 increasing/decreasing strain amplitudes. The resulting block dependent cyclic stress-strain curves was used for estimating the corresponding set of material parameters (elastic modulus, offset yield stress, kinematic hardening parameters $\mathrm{C} 1, \gamma 1)$. However, one of the major challenges is how to estimate the cyclic stress-strain curve for hundreds of fatigue cycles. For the purpose, we developed an automated approach by using AI/ML based TensorFlow library. Once the equivalent cyclic-stress curve estimated, we used our previously developed material modeling approach [17] to estimate the associated block/cycle/time-dependent material parameters. The detail results related to TensorFlow based cyclicstress-strain curve estimation and downstream cyclic-material-parameter estimation are presented below. Before presentation of the results, we briefly discuss the theoretical background of the computationalmechanics-based material modeling approach below.

\subsection{Material Model Theoretical Background}

The FE modeling of reactor components such as welded nozzles requires a knowledge of materialhardening parameters. In our earlier work [17], we developed a detailed methodology for estimating material models. This approach is based on a Chaboche [18]-type material-hardening model to model the stress-strain behavior in elastic regime and regime beyond yield stress. The details of the approach are discussed in our earlier work. However, in this subsection we briefly discuss the basic background behind the model to familiarize readers with the terminology of different parameters.

The stress state of a component can be expressed through the yield function, which is given as

$$
f\left(\boldsymbol{\sigma}^{j}-\boldsymbol{\alpha}^{j}\right)=\sigma^{y}
$$


In Eq. 6.1, $\boldsymbol{\sigma}^{j}$ and $\boldsymbol{\alpha}^{\boldsymbol{j}}$ are, respectively, the total stress and back stress tensor at the $\mathrm{j}^{\text {th }}$ instance, whereas $\sigma^{y}$ is the yield stress. In Eq. 6.1, the back stress $\boldsymbol{\alpha}^{j}$ can be estimated using the linear or nonlinear mapping function of hardening stress (i.e., $\sigma^{j}-\sigma^{y}$ ), with respect to the accumulated plastic strain $\left(\bar{p}=\int_{\mathbf{0}}^{\varepsilon^{p l}} d \varepsilon^{p l}\right)$. The expressions for the linear and nonlinear hardening models are given in Eqs. 6.2 and 6.3 , respectively.

$$
\begin{aligned}
d \alpha^{j} & =\frac{2}{3} C_{1} d \varepsilon^{p l} \\
d \alpha^{j} & =\frac{2}{3} C_{1} d \varepsilon^{p l}-\gamma_{1} \alpha^{j} \bar{p}
\end{aligned}
$$

In Eqs. 6.2 and 6.3, $C_{1}$ and $\gamma_{1}$ are the two material-hardening parameters. These parameters are estimated using numerical optimization such that the $L_{2}$ norm of the incremental parameters (i.e., $\left.\left\|\begin{array}{ll}\| C_{1} & \Delta \gamma_{1}\end{array}\right\|\right)$ is less than a chosen tolerance value. Frist the elastic modulus and offset-strain yield stress have to be estimated using the equivalent-monotonic stress-strain curve. Then the $C_{1}$ and $\gamma_{1}$ parameters have to be estimated based on the plastic portion of the corresponding equivalentmonotonic stress-strain curve. Once the TensorFlow based cyclic-stress-strain curves were estimated, the corresponding up/down-cycle equivalent-monotonic stress-strain curves were estimated. The up/down-cycle monotonic stress-strain curves were used for estimating the corresponding set of material parameters (elastic modulus, offset yield stress, kinematic hardening parameters $\mathrm{C} 1, \gamma 1$ ). The overall process was automatically performed using Python and TensorFlow based material modeling code. The estimated material parameters were automatically stored to a SQL based database that can be shared with US nuclear industry and regulators such as US-NRC on request.

\subsection{Results: Use of TensorFlow to Estimate the Cyclic-Stress-Strain Curves of DMW}

The cyclic stress-strain curves of the DMW were estimated using the ET-F57 fatigue test data. Before predicting the cyclic material parameters, the cyclic-stress-strain curves were estimated using the AI/ML based TensorFlow library and a hyperbolic-tangent based fitting function (refer to section 2.5). First the sensor data imported using pandas - Python Data Analysis Library [19] and cleaned before passing into TensorFlow based cyclic-stress-strain-curve predictor. Figure 6.1 shows the example of repeating one-block (comprising 12 increasing/decreasing cycles) of input strain that was applied to ETF57 DMW filler weld specimen. Whereas, Figure 6.2 shows the corresponding observed (output) stress history. Figure 6.3 shows the example underlying input-output (or strain versus stress hysteresis) data and the corresponding TensorFlow predicted cyclic-stress-strain curve for $1^{\text {st }}$-block of strain loading. Figures 6.4 and 6.5 show the corresponding iteration no. versus estimated bias and loss-function values, respectively. Figure 6.6 shows the corresponding estimated up/down-cycle equivalent-monotonic-stressstrain curve for $1^{\text {st }}$-block of strain loading. The strain versus stress data of the estimated equivalentmonotonic-stress-strain curves were used for the estimation of the material parameters, which related results follow. 


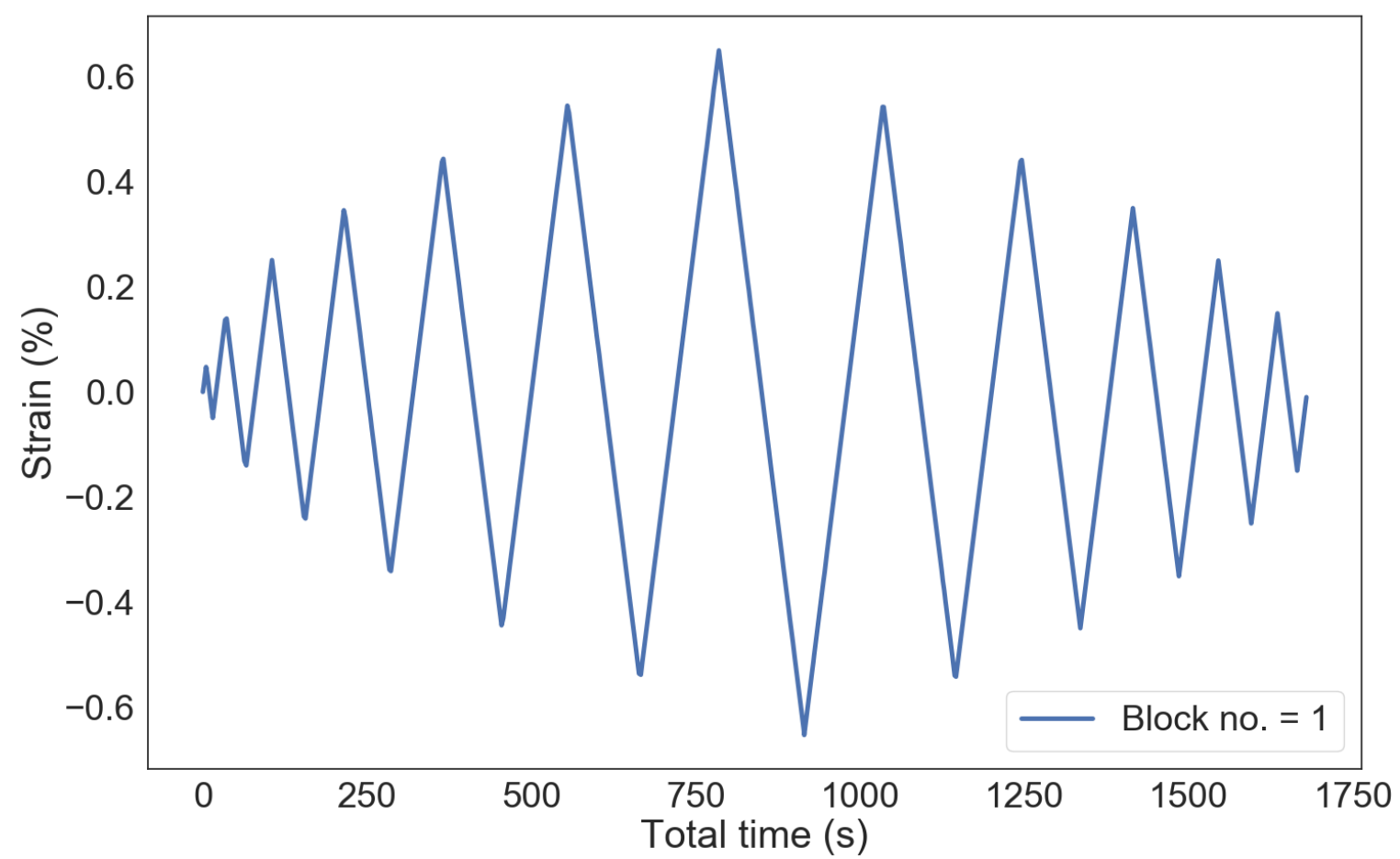

Figure 6. 1 Repeating one-block (comprising 12 increasing/decreasing cycles) of input strain that was applied to ET-F57 DMW filler weld specimen.

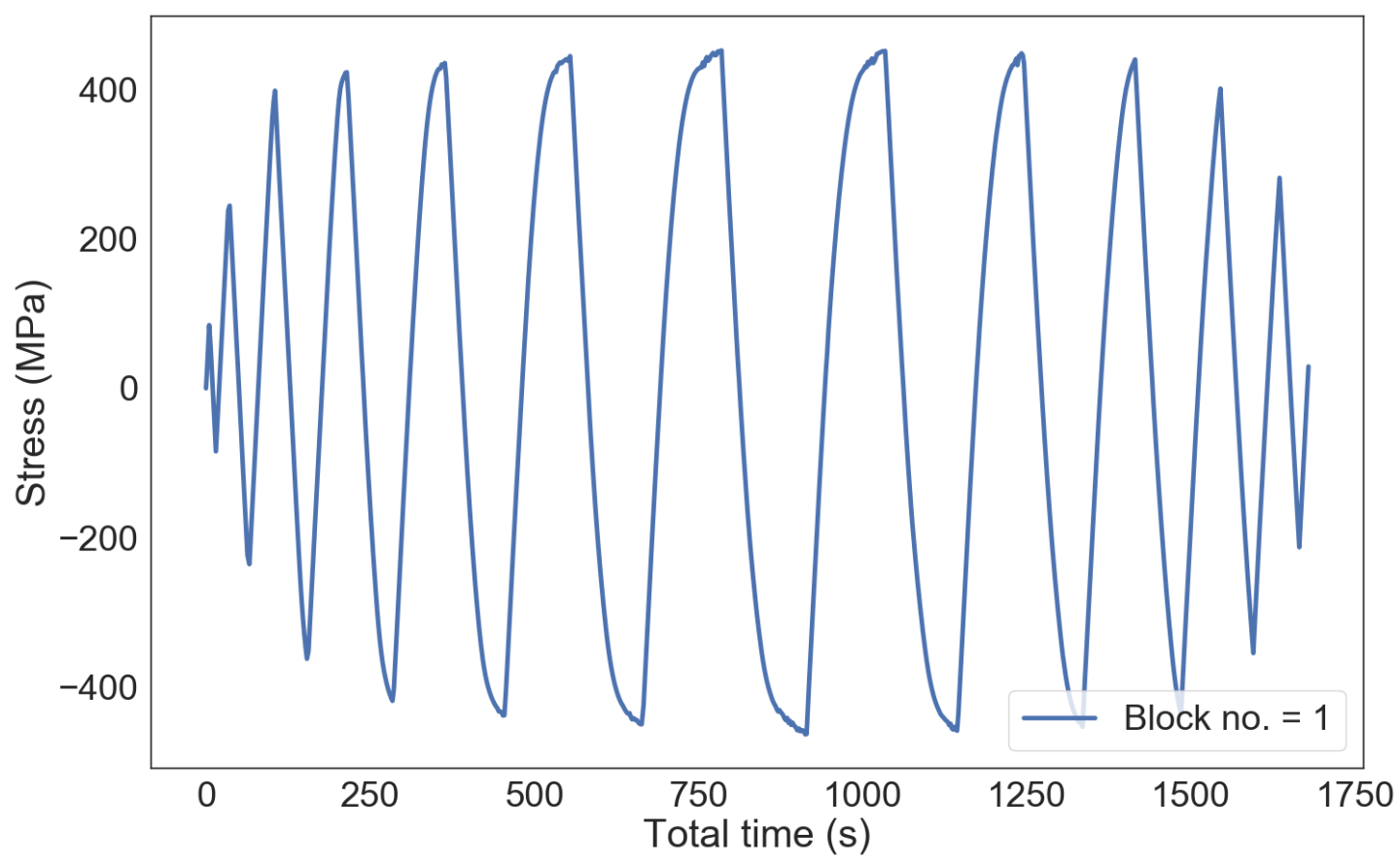

Figure 6. 2 Observed (output) first block (comprising 12 increasing/decreasing cycles) of stress in ETF57 DMW filler weld specimen. 


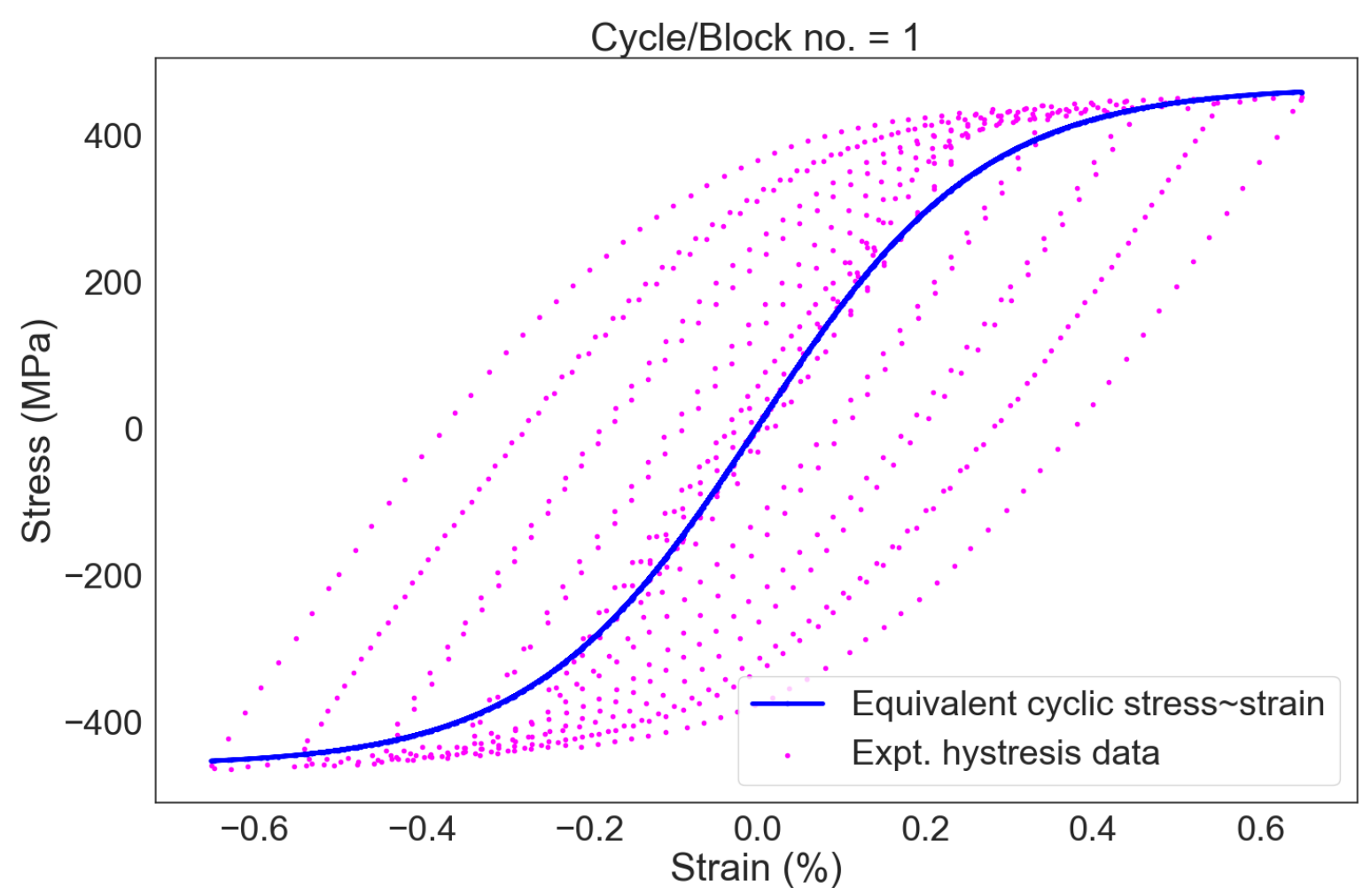

Figure 6. 3 Underlying input-output (or strain versus stress hysteresis) data and the corresponding TensorFlow predicted equivalent cyclic-stress-strain curve for $1^{\text {st }}$-block of strain loading.

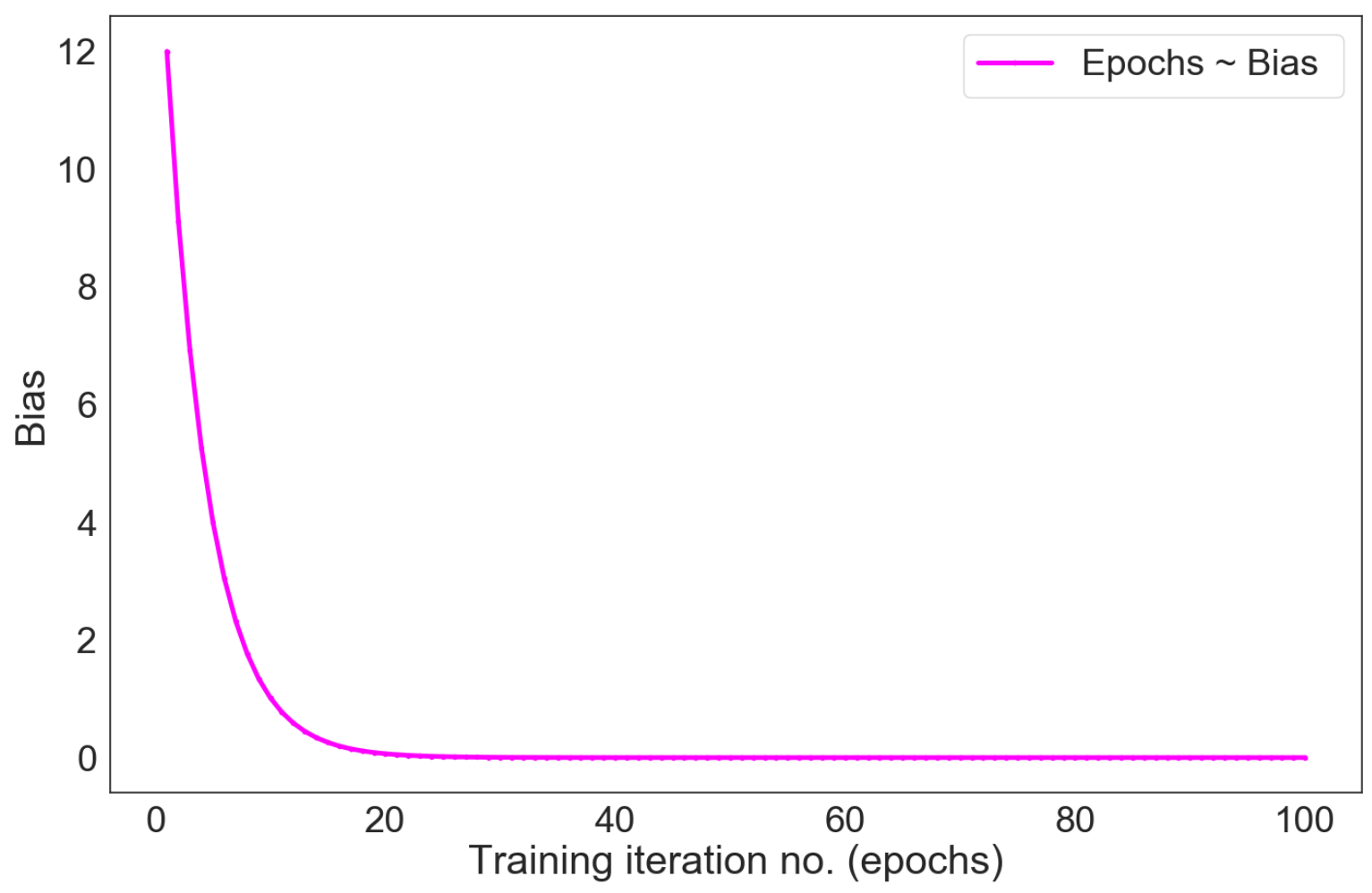

Figure 6. 4 Training iteration no. versus estimated bias for $1^{\text {st }}$-block of strain loading. 


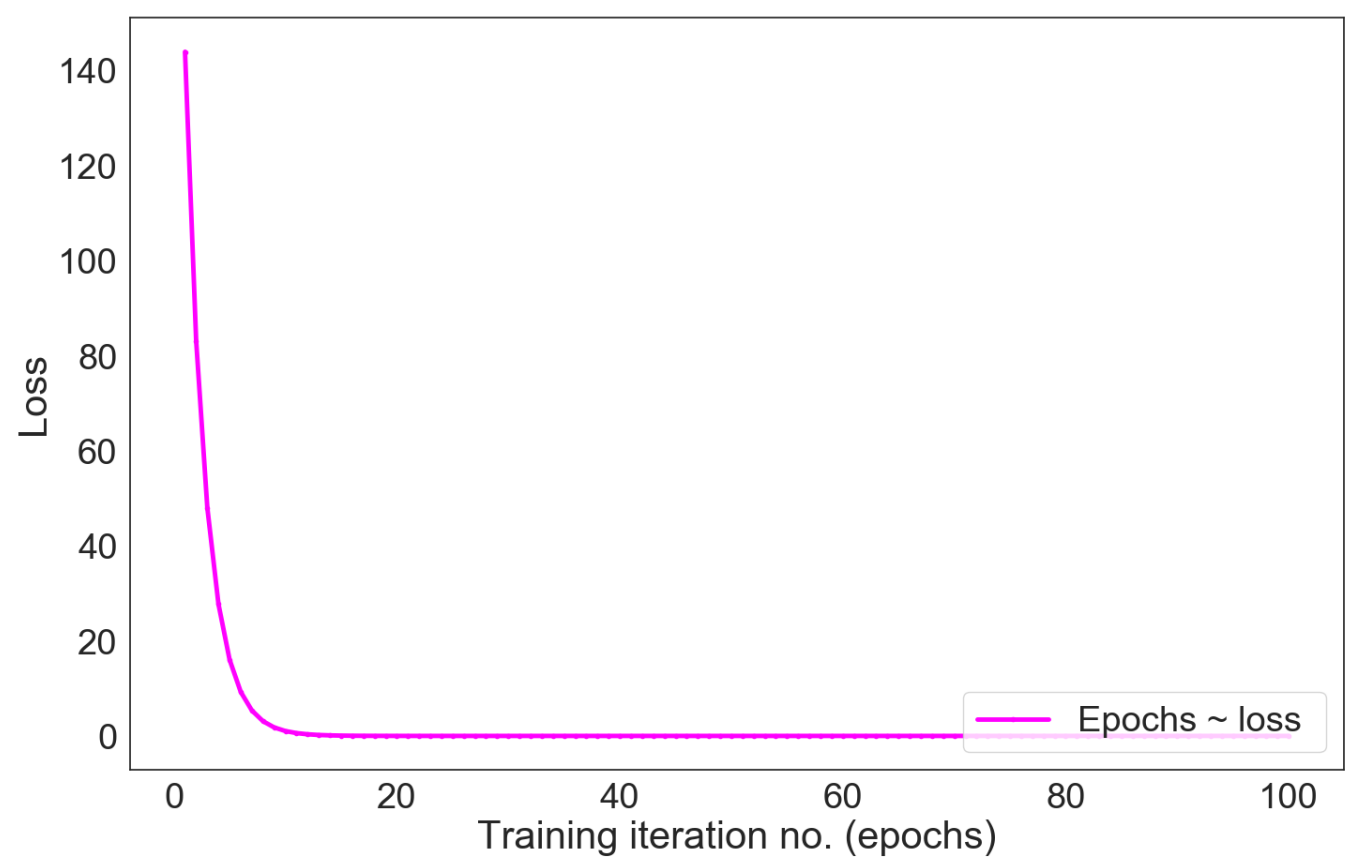

Figure 6. 5 Training iteration no. versus estimated loss function for $1^{\text {st }}$-block of strain loading.

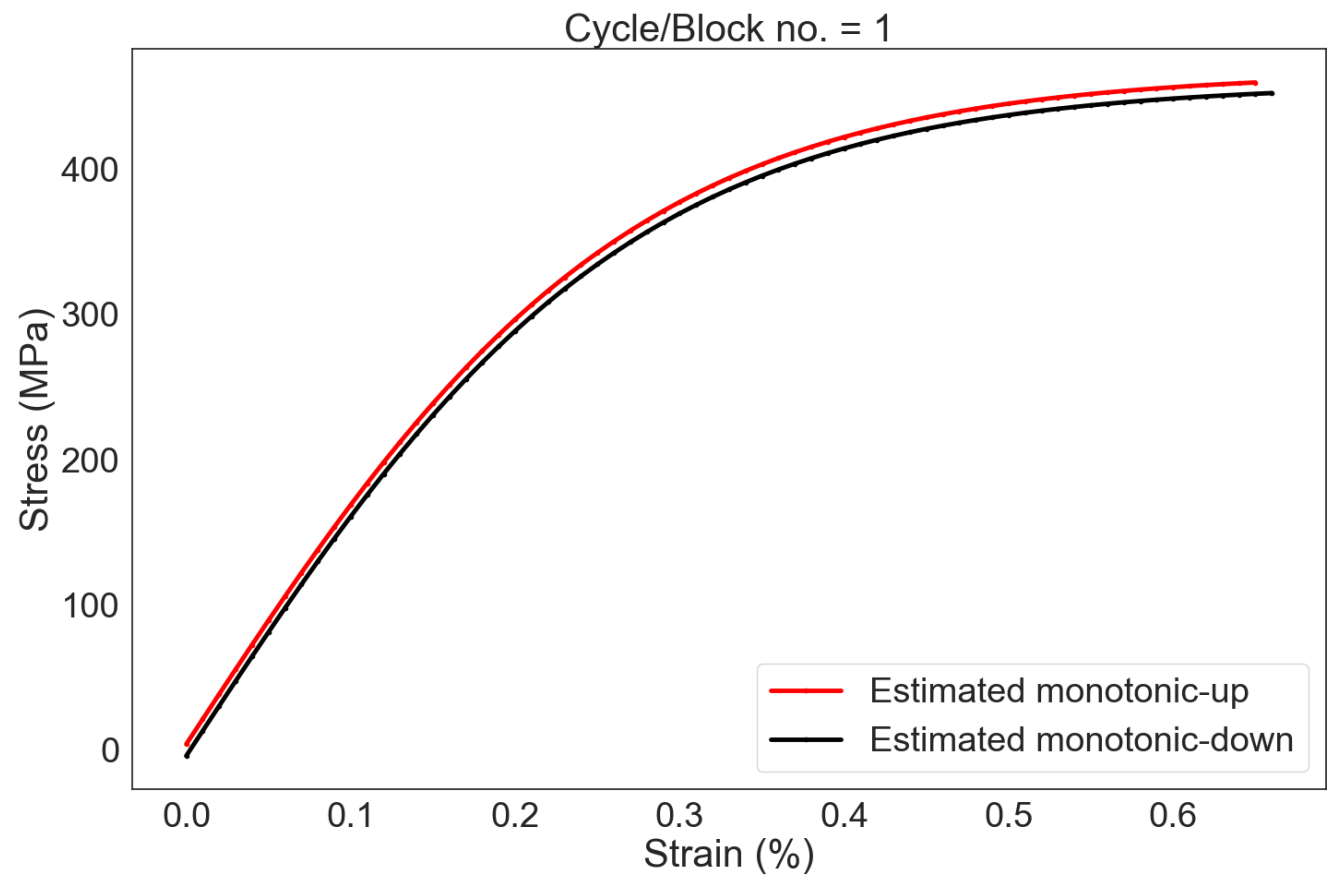

Figure 6. 6 Estimated up/down-cycle equivalent-monotonic stress-strain curve for $1^{\text {st }}$-block of strain loading. 


\subsection{Results: Use of Computational-Mechanics Based Approach to Estimate Elastic-Plastic Material Model Parameters of DMW}

Once, the up/down-cycle equivalent-monotonic-stress-strain curves were estimated, those curves were used for estimating the respective sets of material parameters (elastic modulus, offset yield stress, kinematic hardening parameters $\mathrm{C} 1, \gamma 1$ ). Figure 6.7 shows the example results showing how good/bad the linear and nonlinear kinematic hardening $(\mathrm{KH})$ models capture the plastic strain versus back stress curve of $1^{\text {st }}$-block strain loading. Note that a Newton-based optimization algorithm was used for optimally estimating the kinematic hardening parameters. Whereas the elastic modulus was estimated by least-square fitting of the elastic portion of the combined up/down-cycle equivalent-monotonic-stressstrain curves. The offset-strain yield strains were estimated automatically by using a tangent/slope searching algorithm. Figure 6.8 shows the example iteration no. (in the Newton optimization process) versus estimated $\mathrm{C} 1$ parameter showing convergence of $\mathrm{C} 1$ parameter for $1^{\text {st }}$-block strain loading. Whereas, Figures 6.9 and 6.10 show the corresponding iteration no. (in a Newton optimization process) versus estimated $\gamma 1$ parameter and L2 norm of $\Delta \mathrm{C} 1$ and $\Delta \gamma 1$ showing convergence of estimated parameters for $1^{\text {st }}$-block of strain loading. Similar procedure was followed in an automated loop to estimate the material parameters for the individual block loading of ET-F57 data set. Three sets of time/block dependent parameters were used either assuming $0.05 \%$ or $0.1 \%$ or $0.2 \%$ offset-strain yield stress. A user can use any sets of these parameters but to be consistent. In our analytical modeling cases (discussed in section 7), we used $0.05 \%$ offset-strain material properties to capture maximum plastic regime of the stress-strain curve. Note that capturing more plastic region of stress-strain curve would help in more accurate stress analysis results. Figures 6.11 to 6.16 show the $0.05 \%$ time/block-dependent material model results. The estimated parameters were automatically saved into an easily searchable SQL based database. Figure 6.17 shows the snapshot of the database. This database can be shared with US nuclear industry and regulatory agency on request. Note, that we have also estimated the material parameters with $0.1 \%$ and $0.2 \%$ offset-strain conditions. Those are also saved into the database. 


\subsubsection{Example estimation of material model parameters for $1^{\text {st }}$-block}

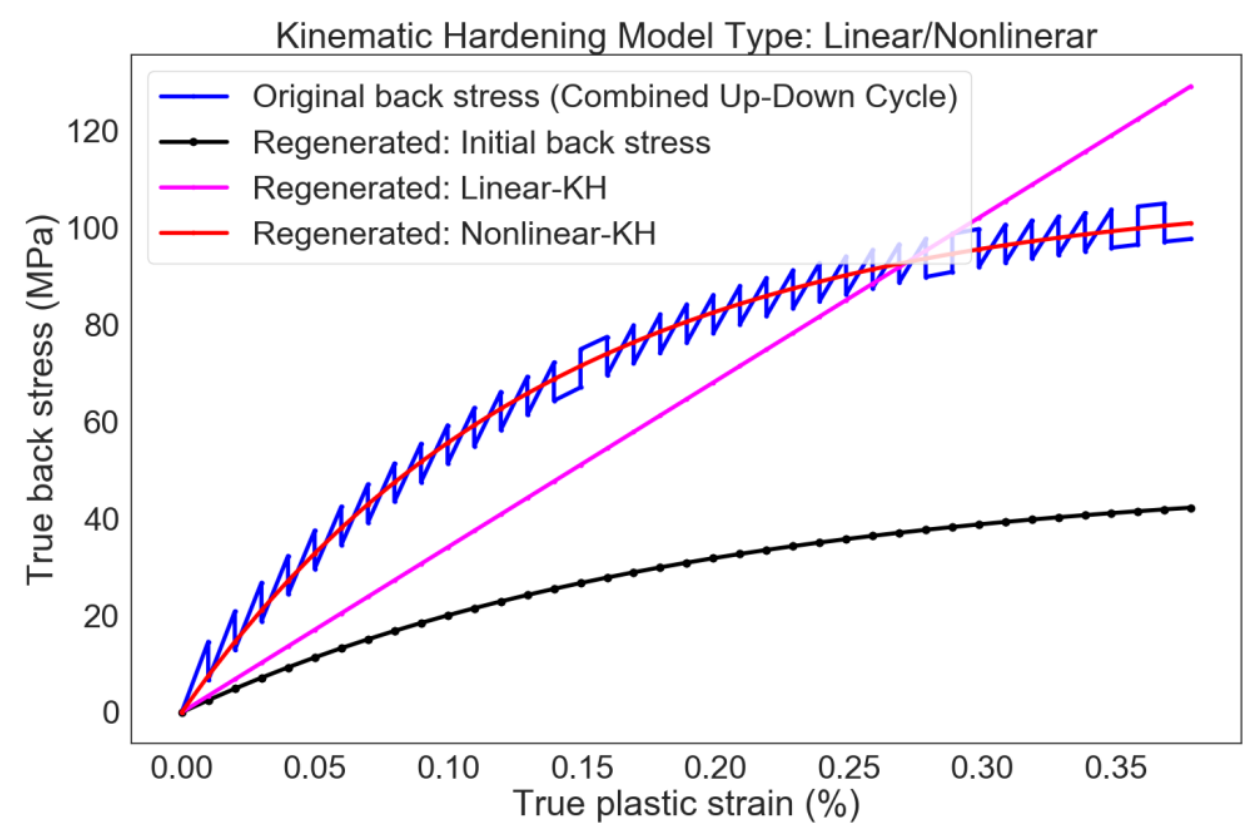

Figure 6. 7 Example results showing how good/bad the linear and nonlinear kinematic hardening $(\mathrm{KH})$ models capture the plastic strain versus back stress curve of $1^{\text {st }}$-block strain loading.

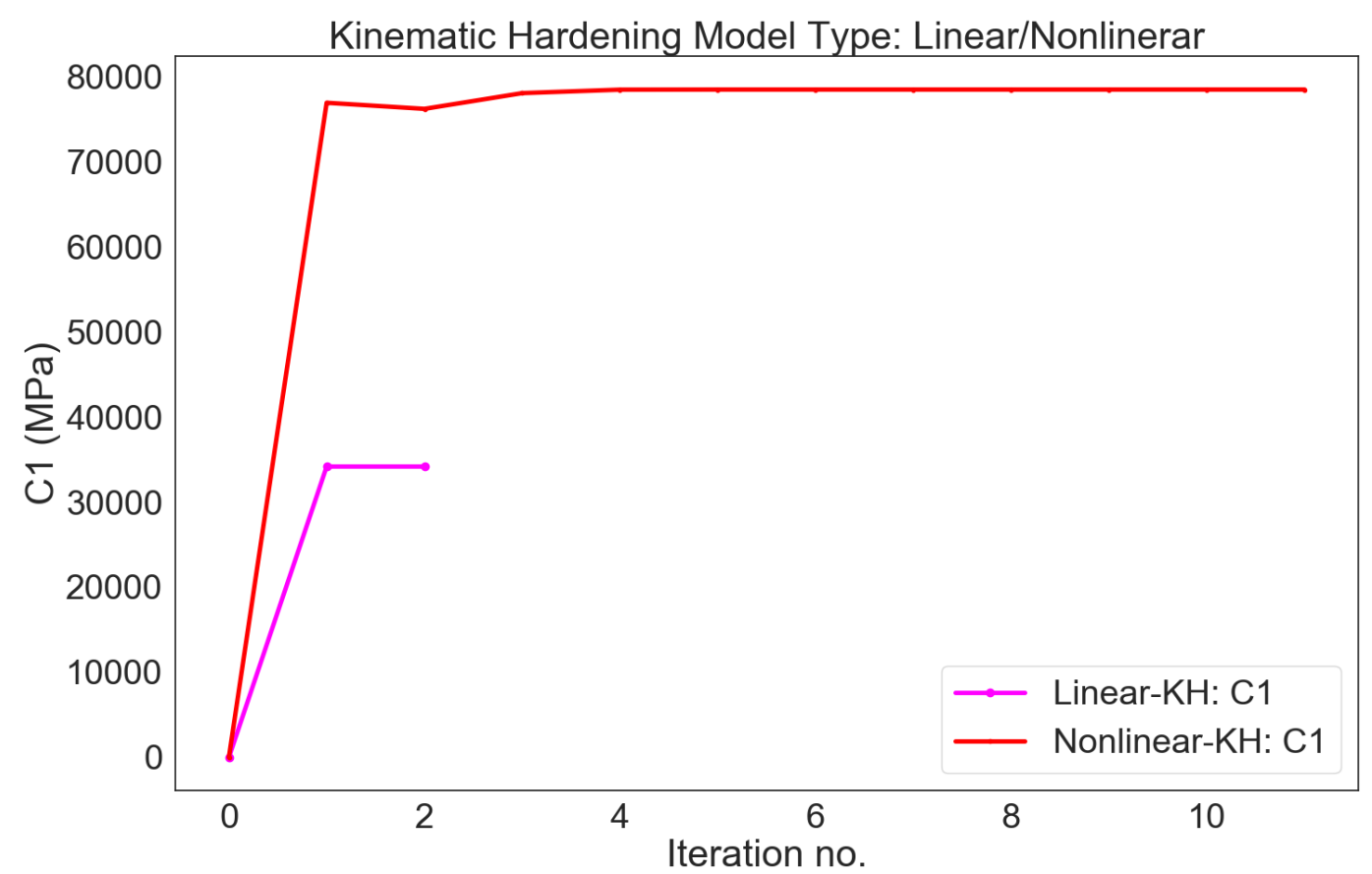

Figure 6. 8 Iteration no. (in a Newton optimization process) versus estimated C1 parameter showing convergence of $\mathrm{C} 1$ parameter for $1^{\text {st }}$-block strain loading. 


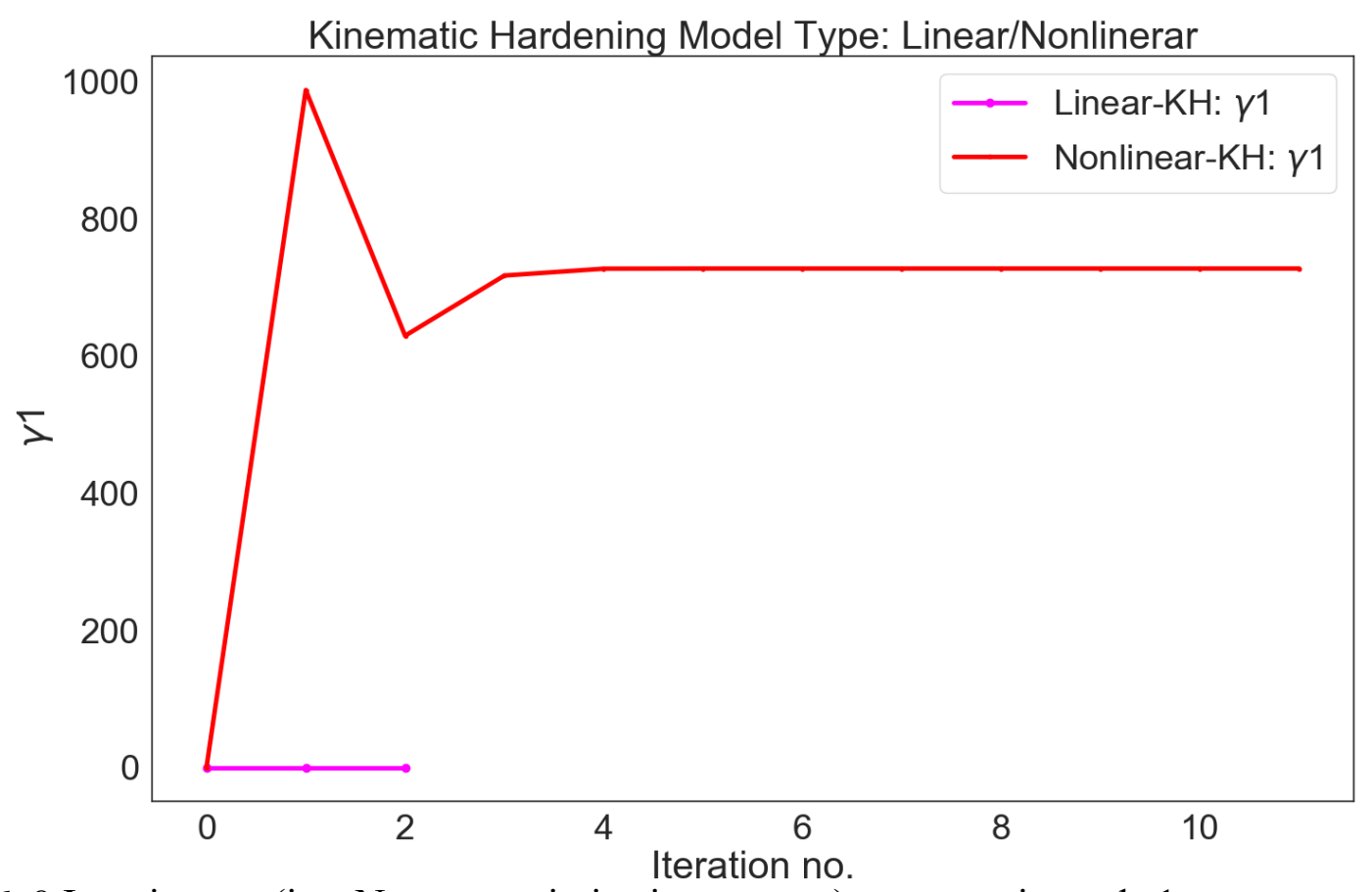

Figure 6. 9 Iteration no. (in a Newton optimization process) versus estimated $\gamma 1$ parameter showing convergence of $\gamma 1$ parameter for $1^{\text {st }}$-block strain loading.

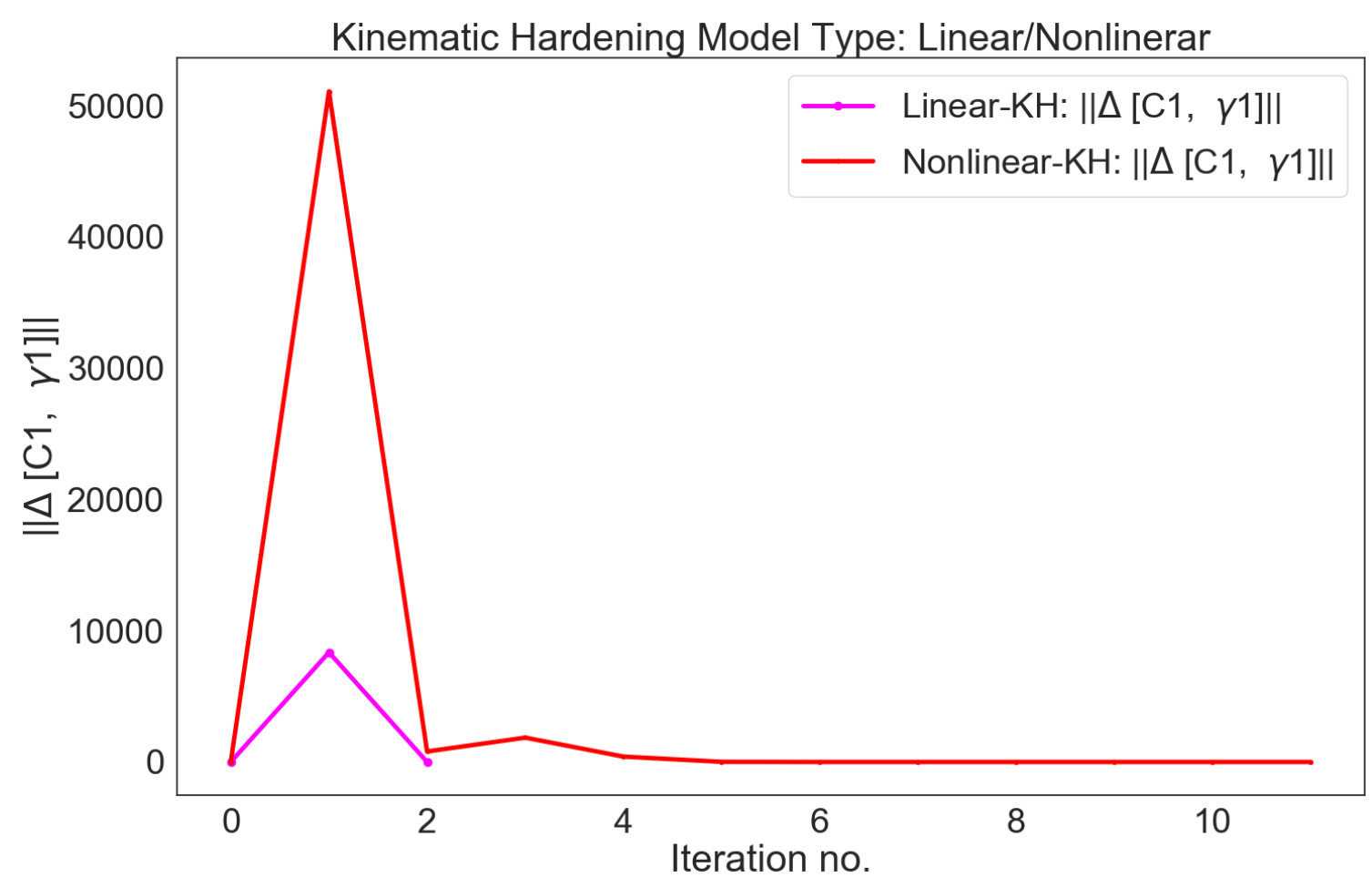

Figure 6. 10 Iteration no. (in a Newton optimization process) versus estimated L2 norm of $\Delta \mathrm{C} 1$ and $\Delta \gamma 1$ showing convergence of estimated parameters for $1^{\text {st }}$-block strain loading. 
6.3.2 Estimated material parameters for entire fatigue life and considering 0.05\% offset yield limit

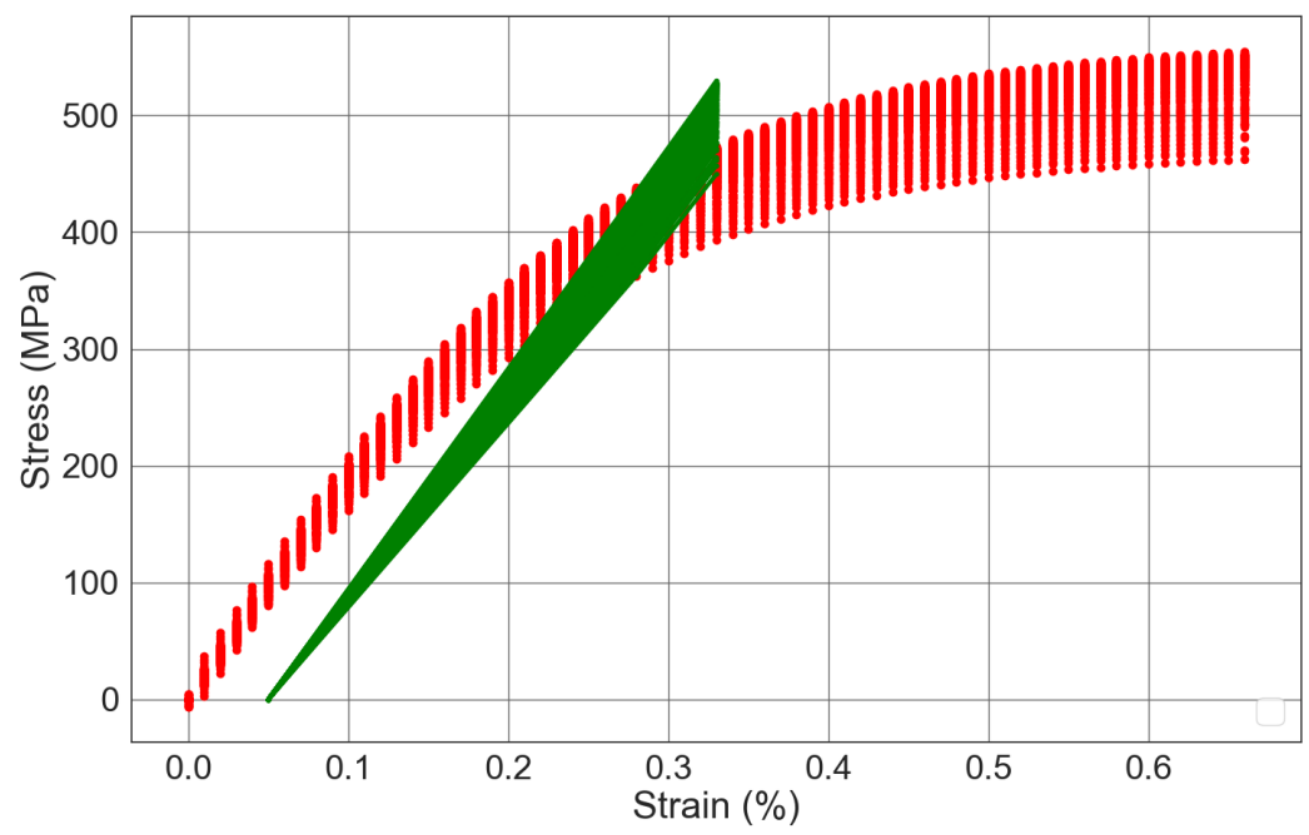

Figure 6. 11 Evolution of equivalent monotonic stress-strain curves (in red) and the corresponding $0.05 \%$ offset yield line (in green) for the entire ET-F57 fatigue life showing the need of cyclic material models.

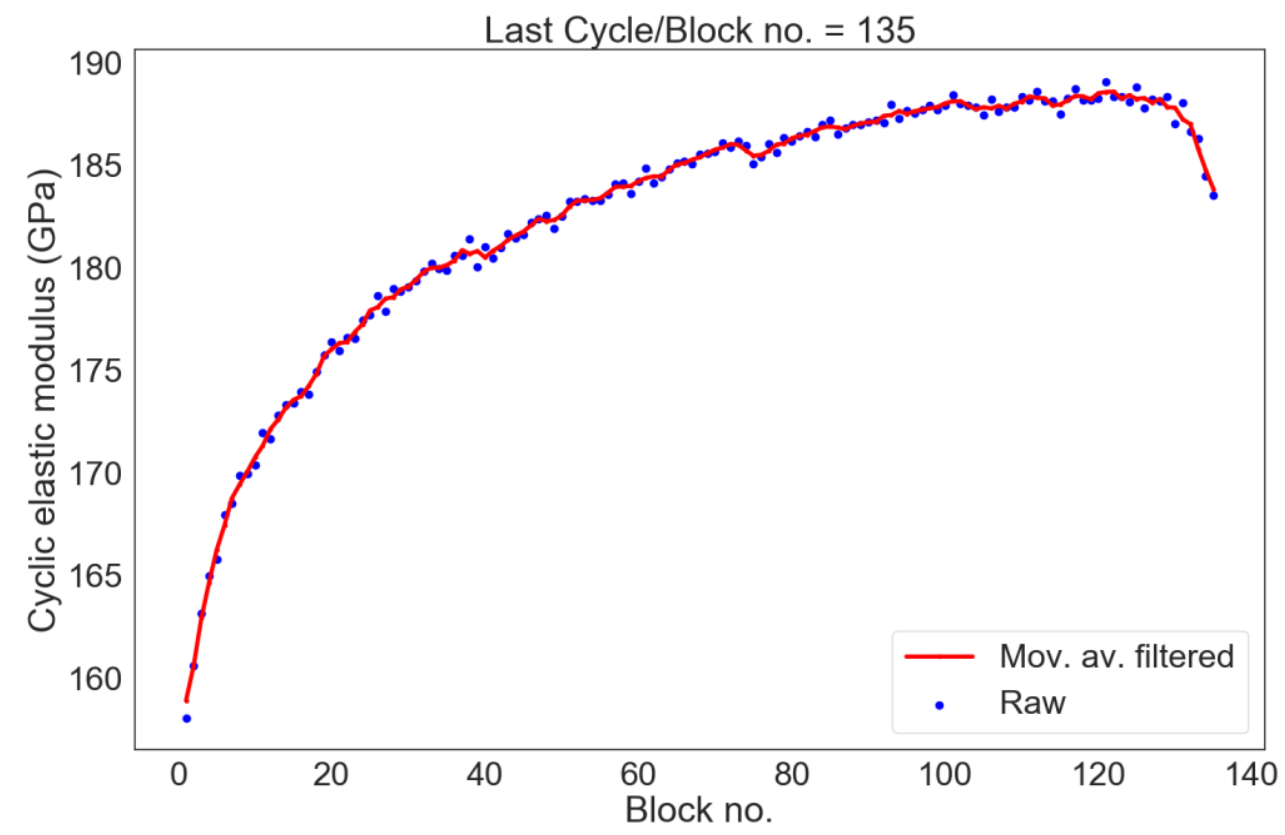

Figure 6. 12 Block no. versus estimated elastic modulus with assumption of $0.05 \%$ offset yield limit. 


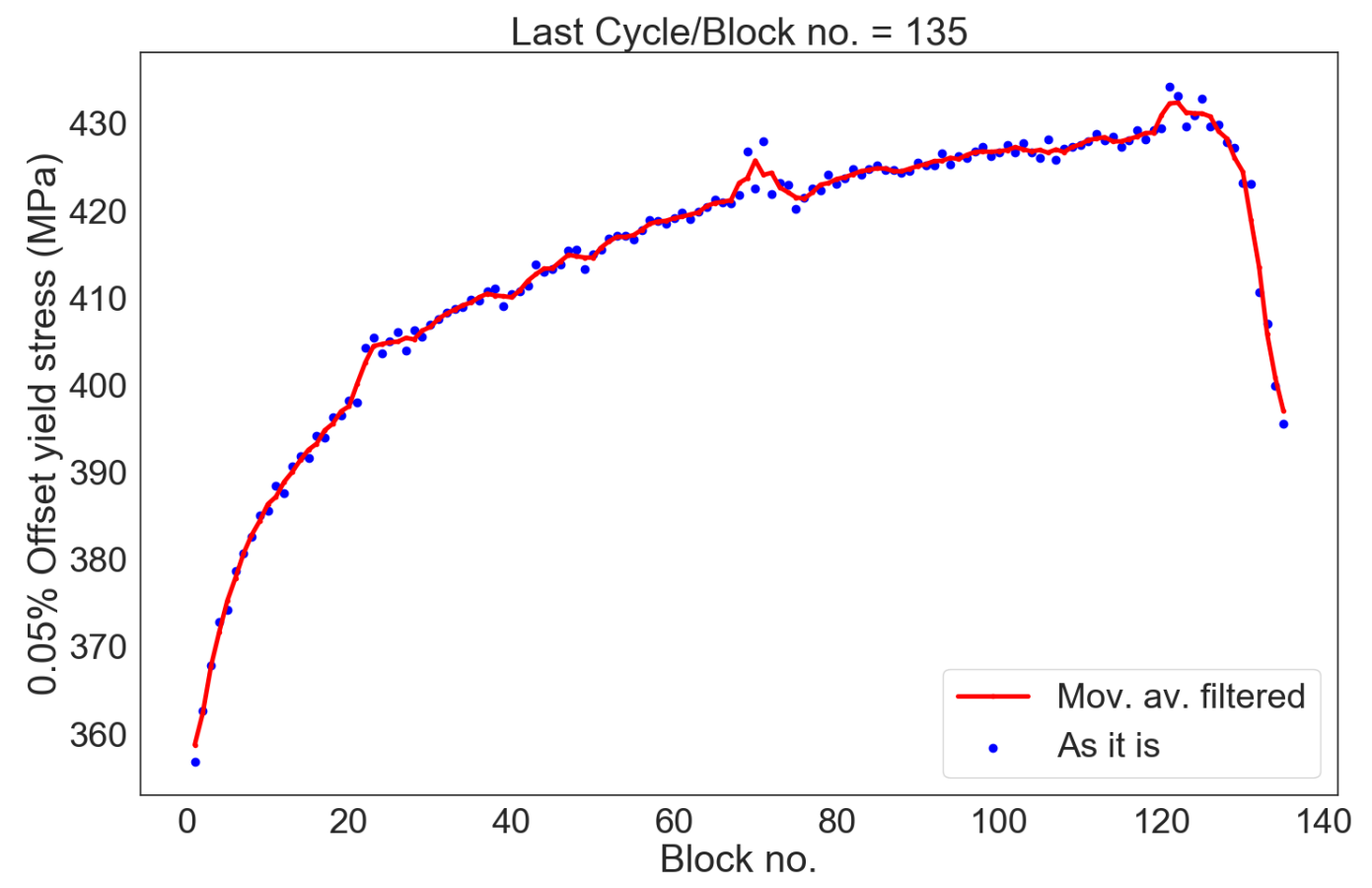

Figure 6. 13 Block no. versus estimated offset yield stress with assumption of $0.05 \%$ offset yield limit.

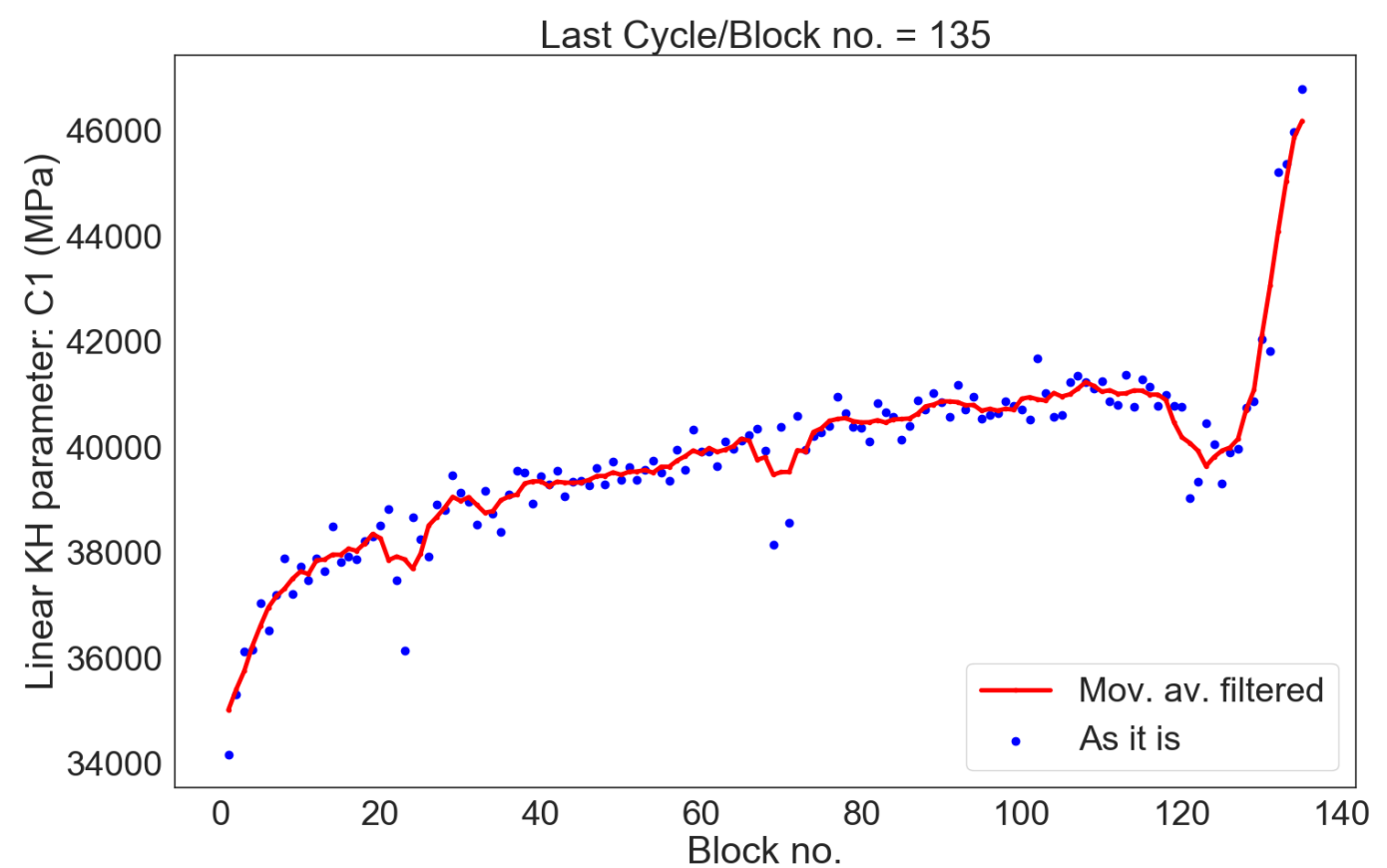

Figure 6. 14 Block no. versus estimated linear kinematic hardening parameter $\mathrm{C} 1$ with assumption of $0.05 \%$ offset yield limit. 


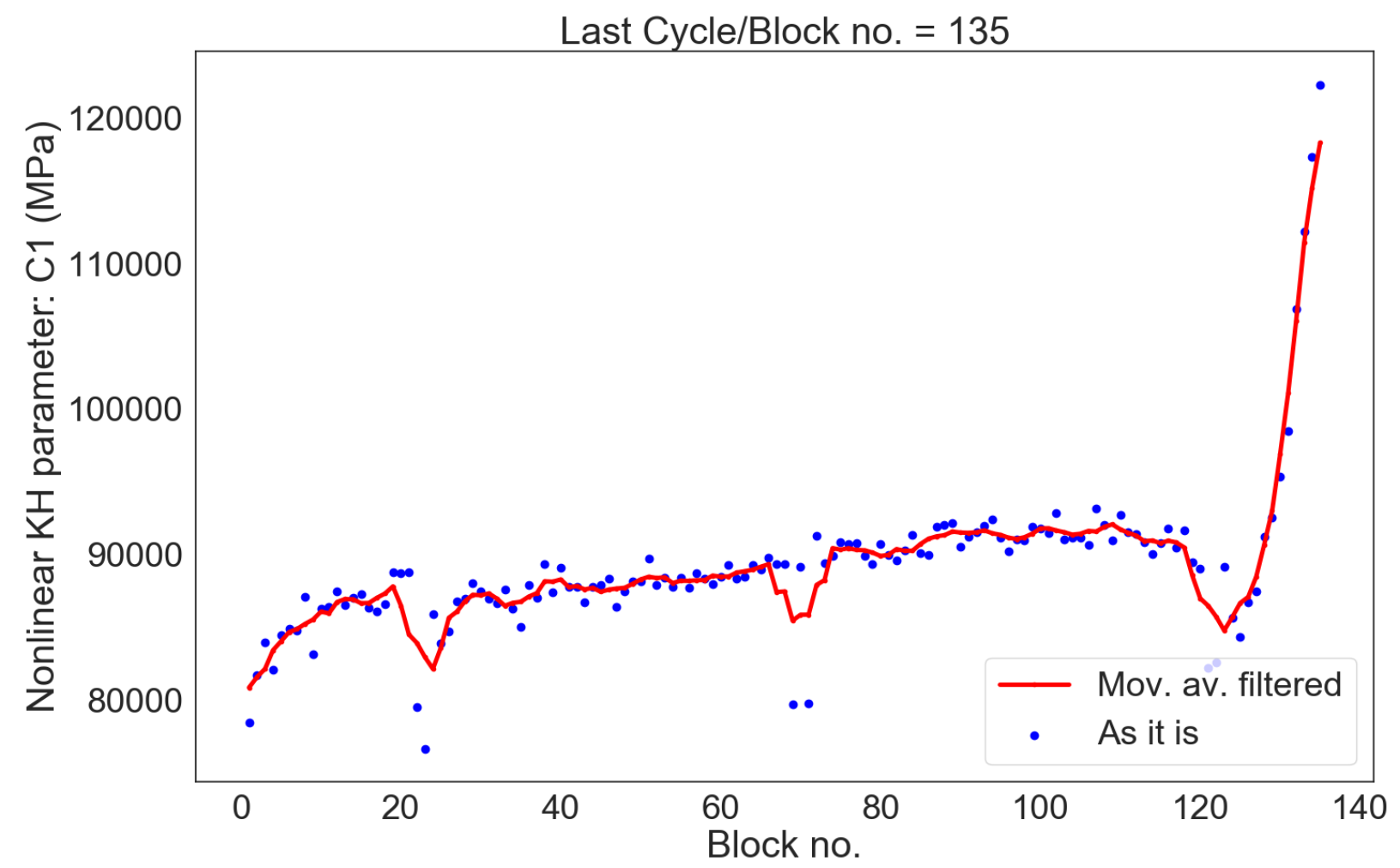

Figure 6. 15 Block no. versus estimated nonlinear kinematic hardening parameter $\mathrm{C} 1$ with assumption of $0.05 \%$ offset yield limit.

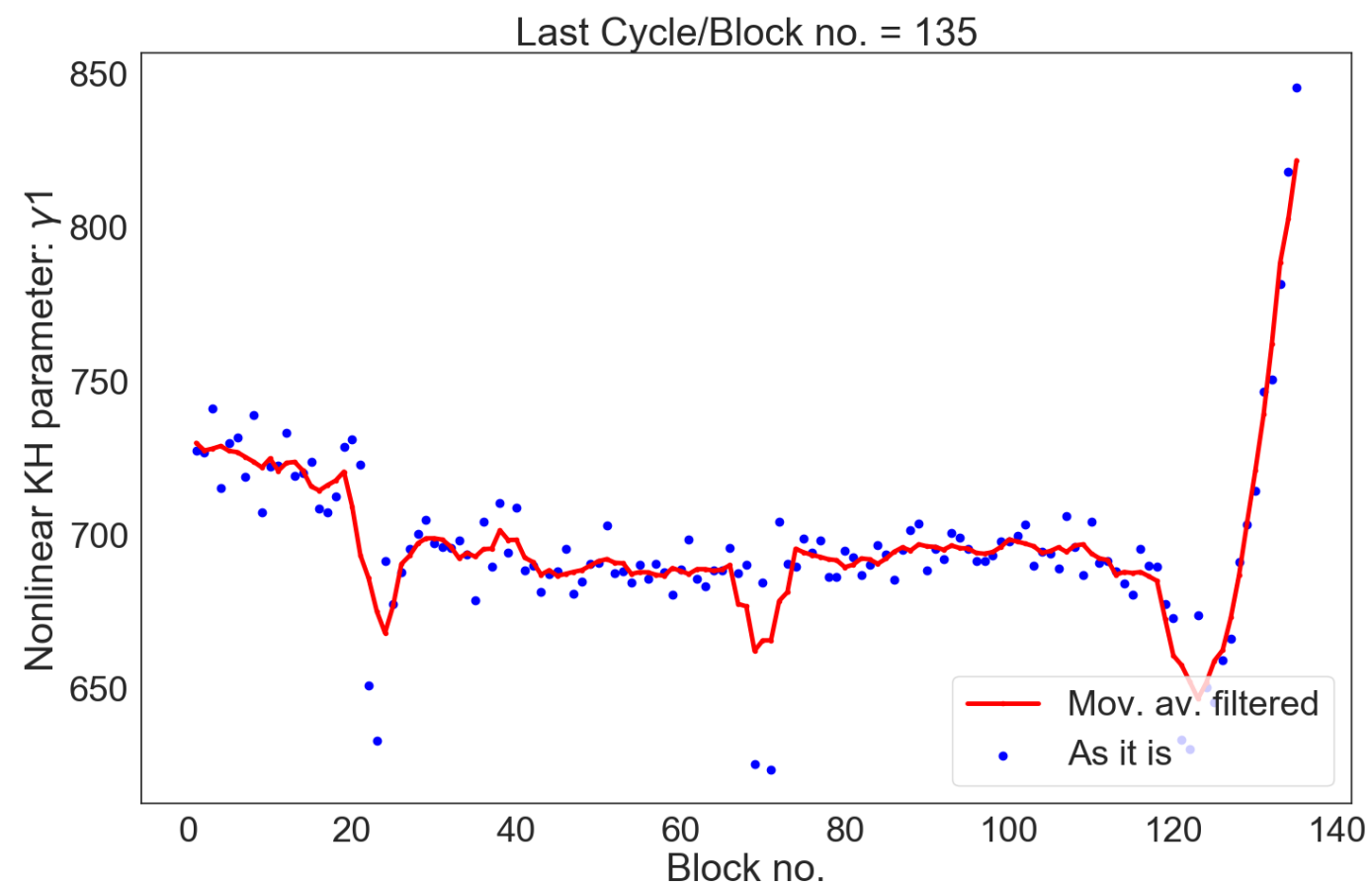

Figure 6. 16 Block no. versus estimated nonlinear kinematic hardening parameter $\gamma 1$ with assumption of $0.05 \%$ offset yield limit. 


\subsubsection{Automated material parameter data saving into SQL database}

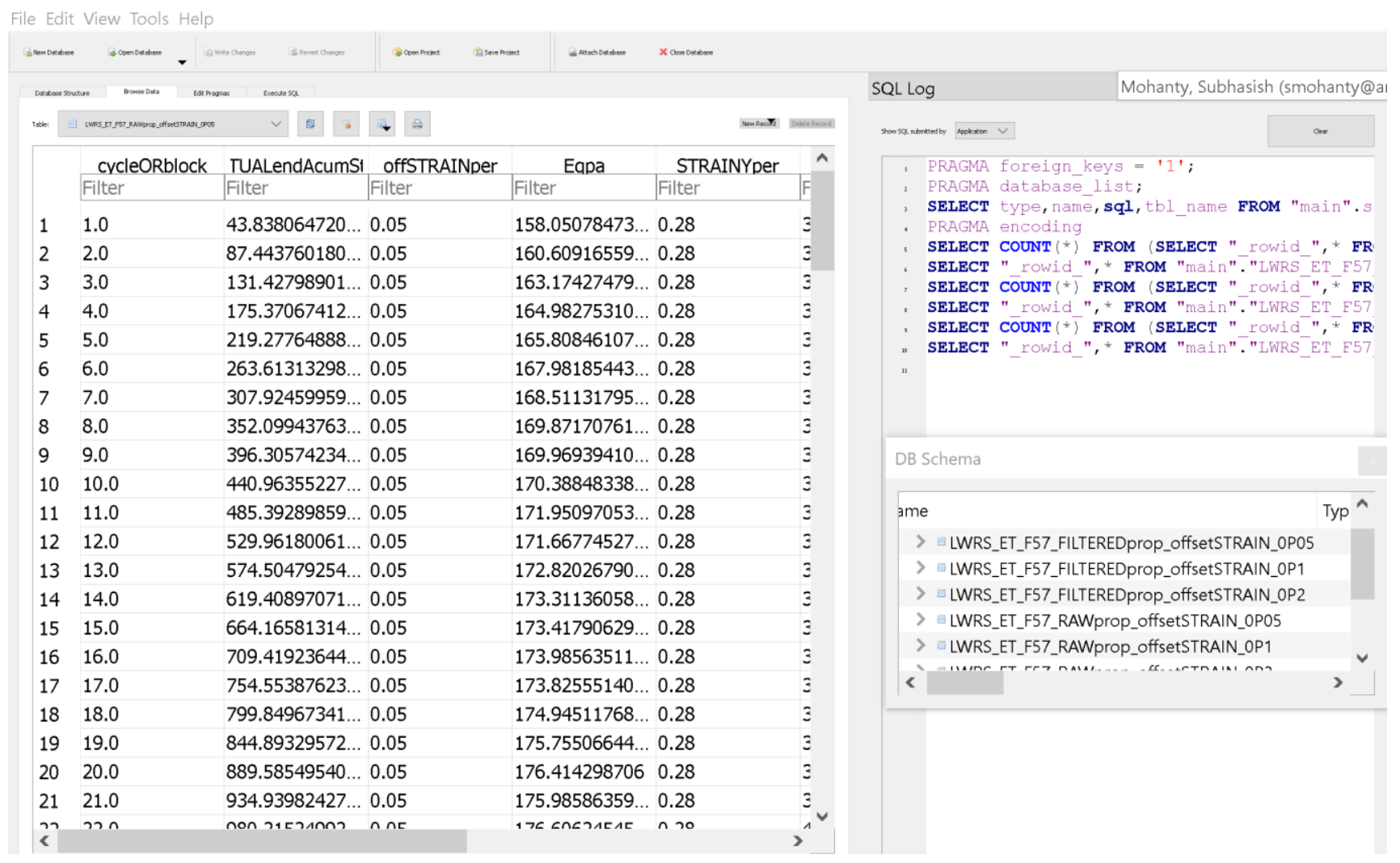

Figure 6. 17 Snapshot of the automatically updated SQL based material database. 


\section{Analytical Model Based Cyclic Stress Analysis of Different DMW Test Cases under Constant and Variable Amplitude Cyclic Loading}

In this section, we present the results related to analytical model based cyclic stress analysis of different DMW test cases. The purpose is to validate the cyclic plasticity material models discussed in section 6. The discussed analytical model is to capture the yield function given in Eq. 6.1. If the analytical model can show that the developed material model and related constitutive relations can accurately predict the stress, then the material model can be used for the stress analysis of 3D reactor components. The analytical model was tested for experimental test cases with intended strain amplitude either equal to or close to $0.6 \%$. In our earlier work [17], we performed 3D finite element (FE) model based thermal-mechanical stress analysis of PWR surge-line nozzle and pressurizer-bottom-head assembly. This 3D FE model predicted a limiting strain range of 1 to $1.4 \%$ in the DMW region of the surge-line nozzle. This leads to a strain amplitude of 0.5 to $0.7 \%$ under most limiting displacement boundary conditions. The selection of test case amplitude of $0.6 \%$ is motivated from the abovementioned 3D stress analysis results of PWR surge-line nozzle. Following test cases (refer to section 3 for more details) were tested:

1) ET-F57 DMW specimen subjected to gauge-area-strain-control based variable amplitude strain loading.

2) ET-F59 DMW specimen subjected to gauge-area-strain-control based constant amplitude strain loading.

3) ET-F60 DMW specimen subjected to usual pull-rod-control based constant amplitude displacement loading.

4) ET-F61 DMW specimen subjected to AI-guided and pull-rod-control based constant amplitude displacement loading.

For each of the above-mentioned test cases, the analytical model was tested against both fixed tensile-test based properties and the time-dependent material models presented in section 6 . While using time-dependent material models for all the above test cases we considered time/cycle/block as field variable to choose the respective set of material parameters (elastic modulus, yield stress and kinematic hardening parameters $\mathrm{C} 1$ and $\gamma 1$ ). Also, we used $0.05 \%$ offset-strain based material parameters for all the test cases discussed in this section. The material model parameters were estimated using the data from ET-F57 fatigue test as described in section 6. We used the same material model for totally different test cases (ET-F59, ET-F60 and ET-F61) in addition to testing the ET-F57 case. This is to check the universality of the developed material model. While selecting the material properties for ET-F59, ETF60 and ET-F61 test cases we scaled the fatigue cycle of these test cases to match with the ET-F57 fatigue cycle number. In addition to the scaled-cycle-property based analytical models we also developed an accumulated-strain-energy dependent material models for which the time-dependent material properties have to be picked based on the accumulated strain energy of the component at a given time/cycle/block. However, the accumulated-strain-energy dependent material model was tested for the ET-F57 test case only. The other test case will be verified in our future work. As can be seen from respective results, for ET-F57 test case we found the accumulated-strain-energy dependent material model gives better accuracy compared to the cycle dependent material models. However, the accumulated-strain-energy dependent material model need to be further tested. In addition to the fixed- 
tensile-property based analytical model (for ET-F57, ET-F59, ET-F60 and ET-F61 test cases) results, the results for the above mentioned four scaled-cycle-property based analytical models (for ET-F57, ETF59, ET-F60 and ET-F61 test cases) and one accumulated-strain-energy dependent analytical model (for ET-F57 test case) are presented below. From the below shown results, it can be found that the, cyclicplasticity based constitutive relation and associated analytical model can predict the cyclic stress not only under constant amplitude loading but also under variable amplitude loading with load-sequence effect. The results show that conventional tensile-test-based fixed material parameters wrongly predict the stress (both amplitude and hysteresis behavior) under cyclic loading. This necessitate the use of cyclic plasticity-based models for more accurate life estimation of reactor components.

\subsection{Cyclic Stress Analysis Results for ET-F57 DMW Specimen Subjected to Gauge-area-strain-control Based Variable Amplitude Strain Loading}

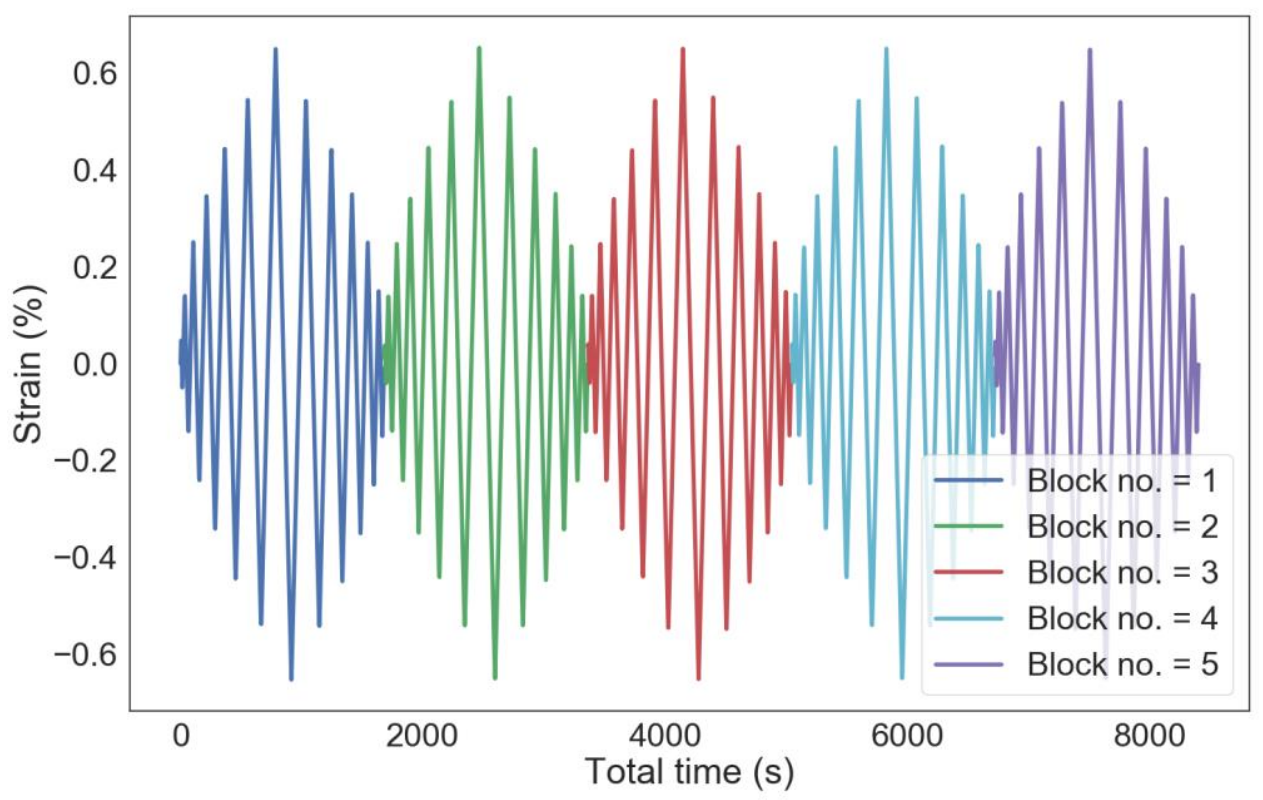

Figure 7. 1 Example first 5 blocks ET-F57 input strain loading. 


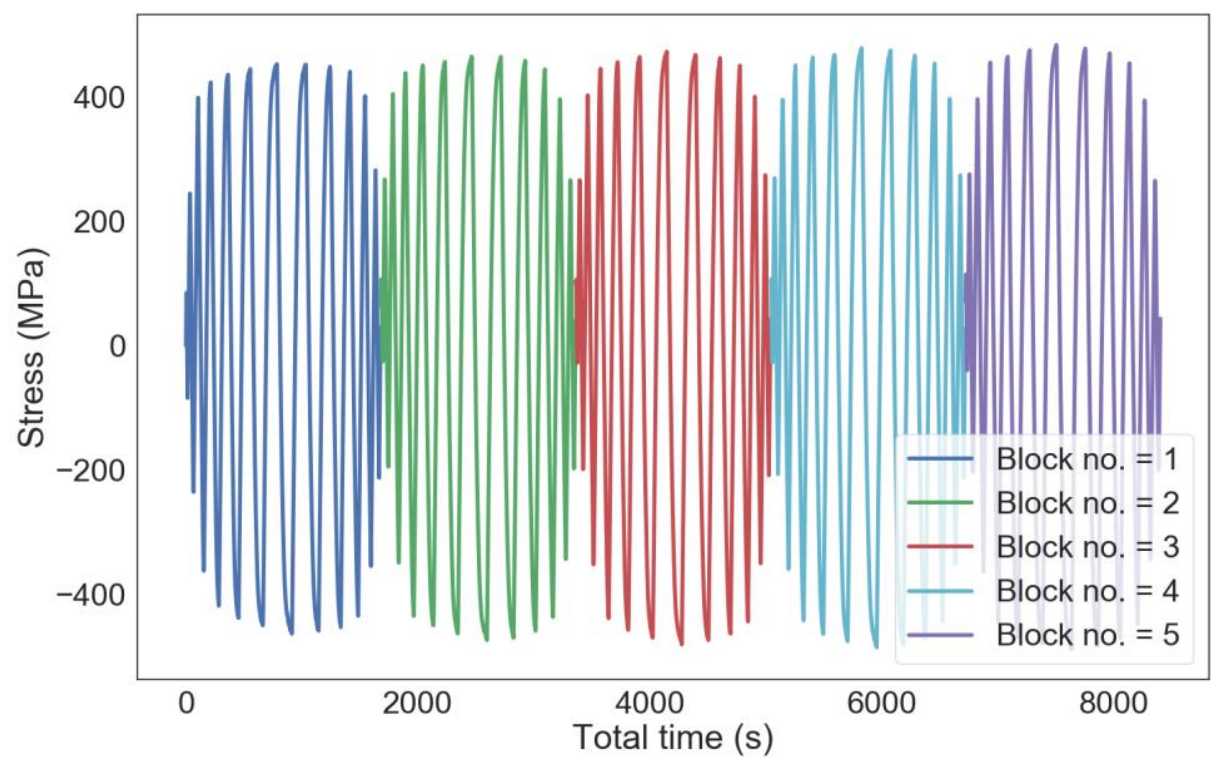

Figure 7. 2 Example first 5 blocks ET-F57 observed stress.

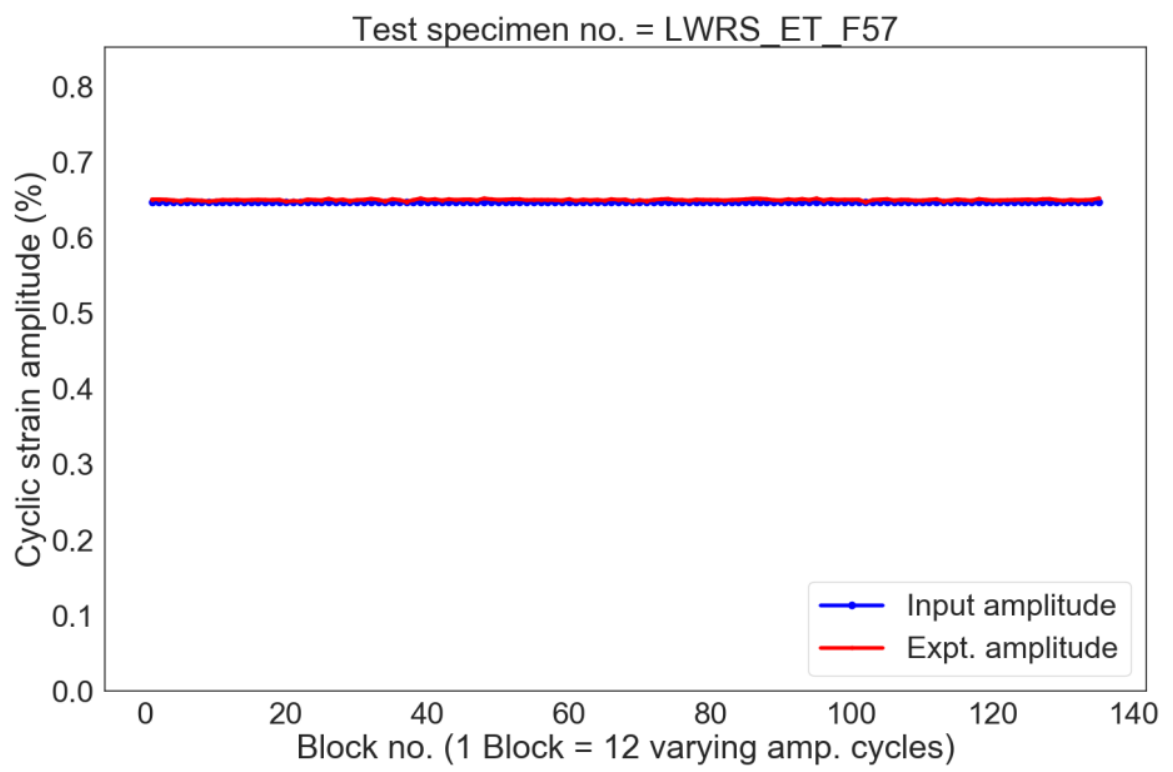

Figure 7. 3 Block no. versus maximum cyclic strain amplitude (both applied to analytical model and experimentally observed during the fatigue test of ET-F57 specimen) to track any anomaly in strain loading. 
7.1.1 Cyclic stress analysis results for ET-F57 specimen using fixed tensile properties

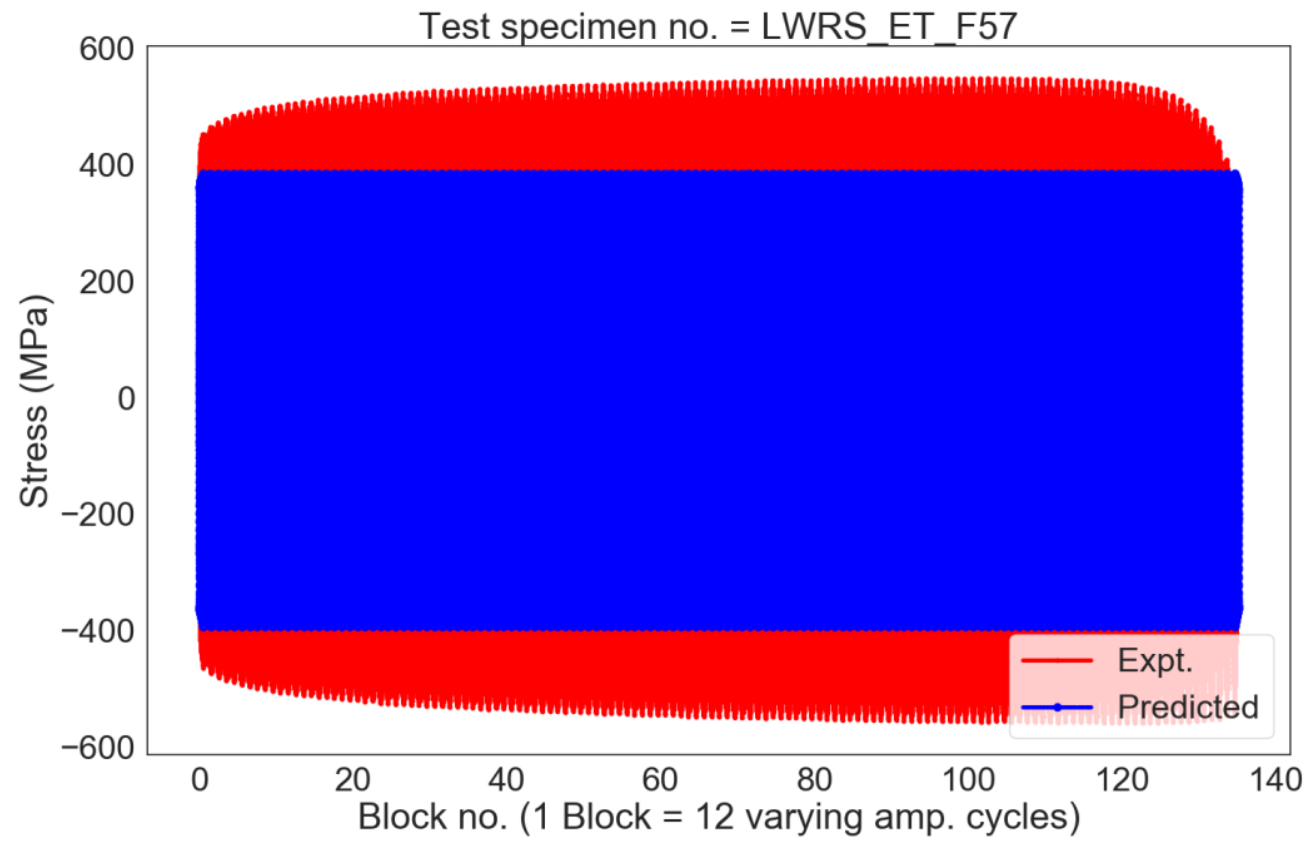

Figure 7. 4 Fixed tensile properties based predicted versus experimentally observed cyclic stress for the entire fatigue life of ET-F57 test case.

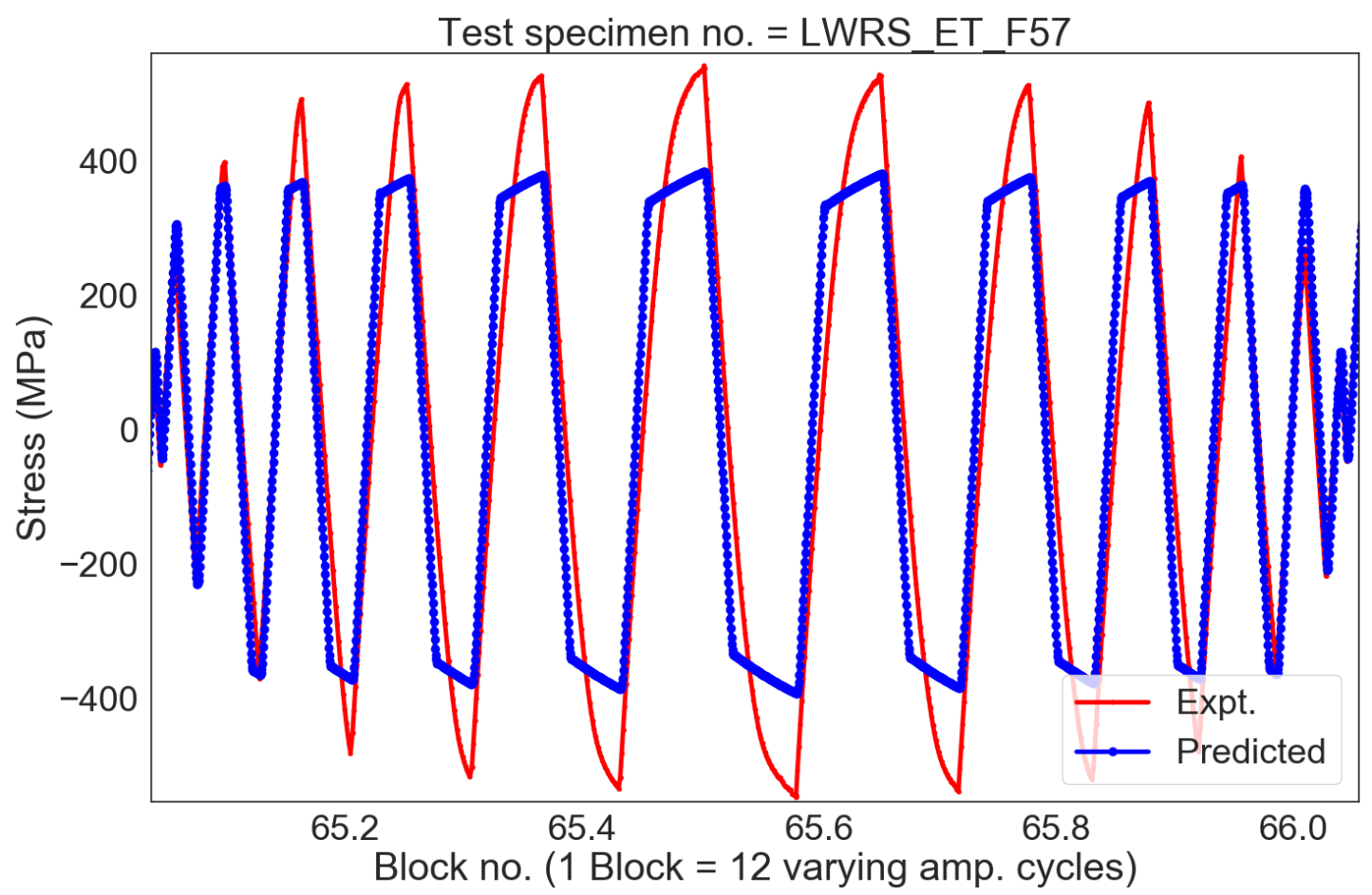

Figure 7. 5 Magnified version of Figure 7.4, approximately during the half-life of ET-F57 specimen. 


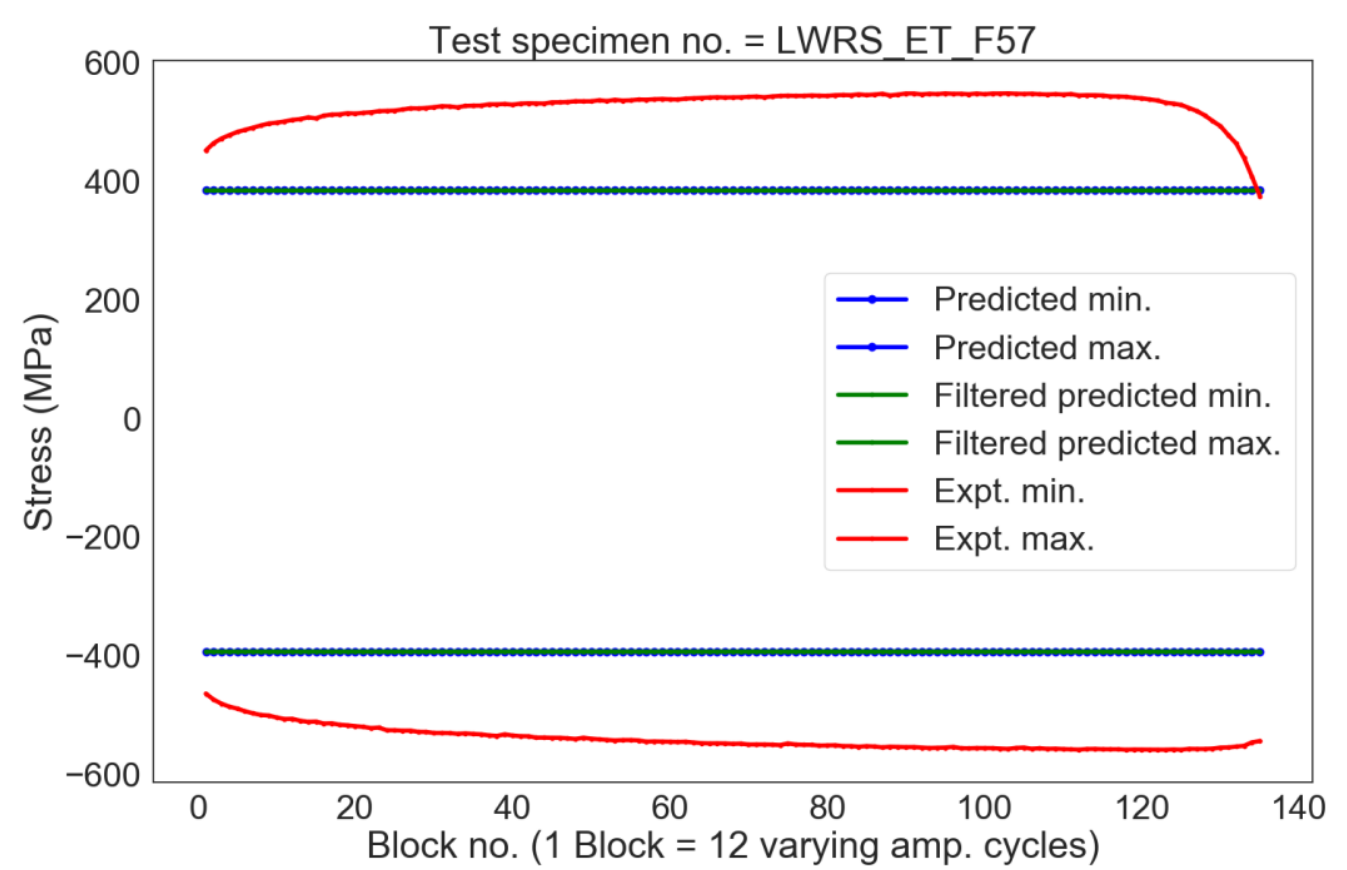

Figure 7. 6 Fixed tensile properties based predicted versus experimentally observed max./min stressamplitudes for the entire fatigue life of ET-F57 test case (The filtered data is to remove any anomaly associated with model prediction).

7.1.2 Cyclic stress analysis results for ET-F57 specimen using variable scaled-cycle-dependent material model properties

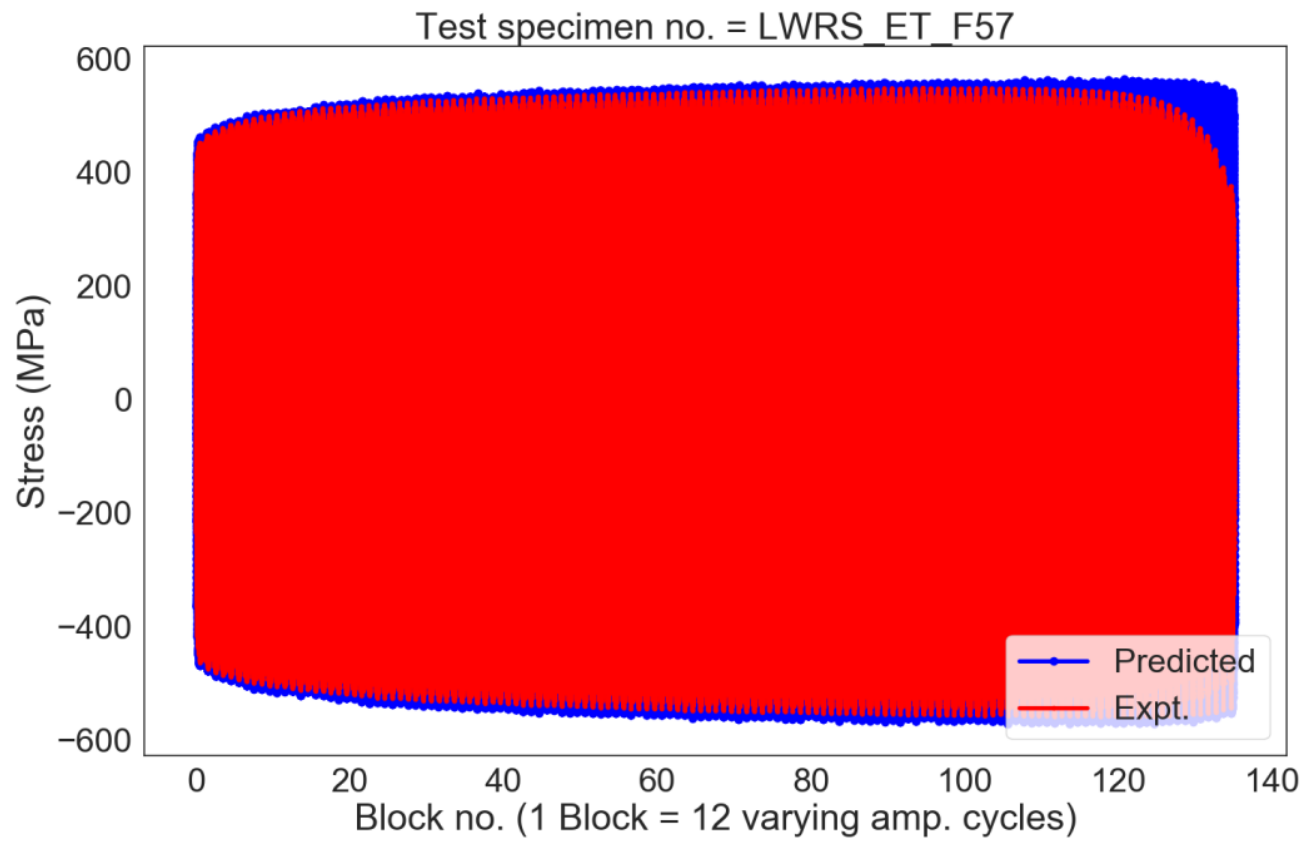

Figure 7. 7 Cycle-dependent properties based predicted versus experimentally observed cyclic stress for the entire fatigue life of ET-F57 test case. 


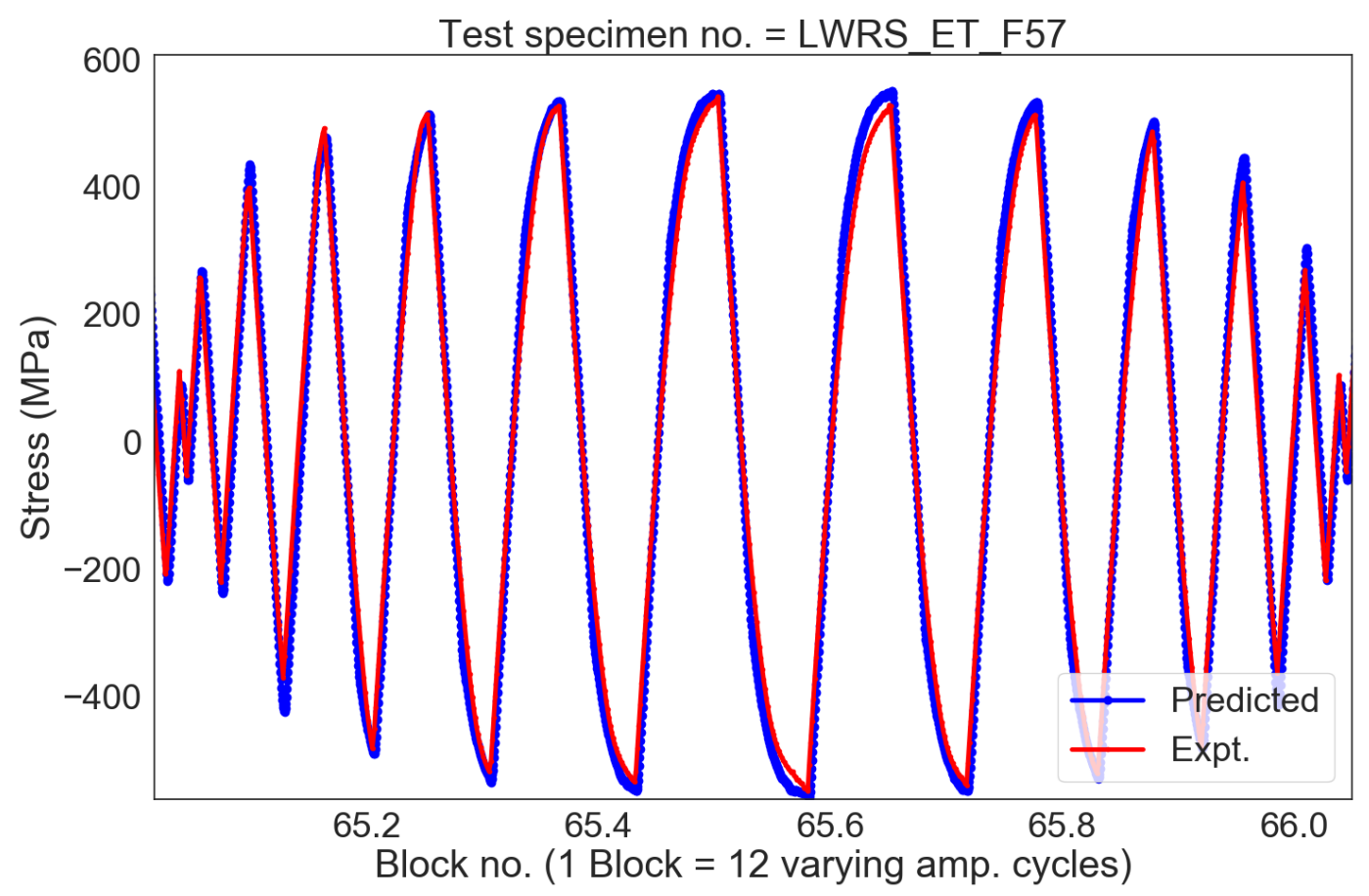

Figure 7. 8 Magnified version of Figure 7.7, approximately during the half-life of ET-F57 specimen.

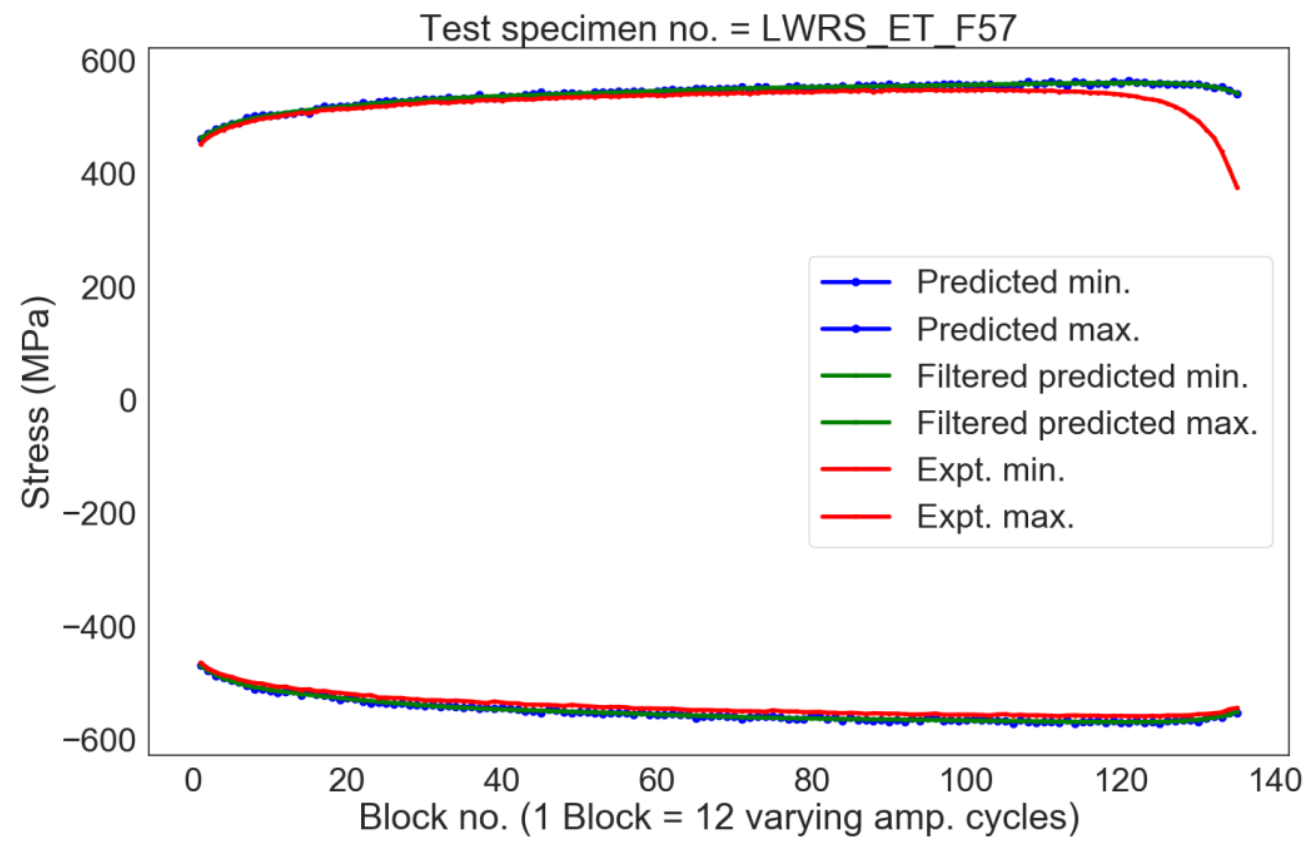

Figure 7. 9 Cycle-dependent properties based predicted versus experimentally observed max./min stressamplitudes for the entire fatigue life of ET-F57 test case. 


\subsection{Cyclic Stress Analysis Results for ET-F59 DMW Specimen Subjected to Gauge-area-strain- control Based Constant Amplitude Strain Loading}

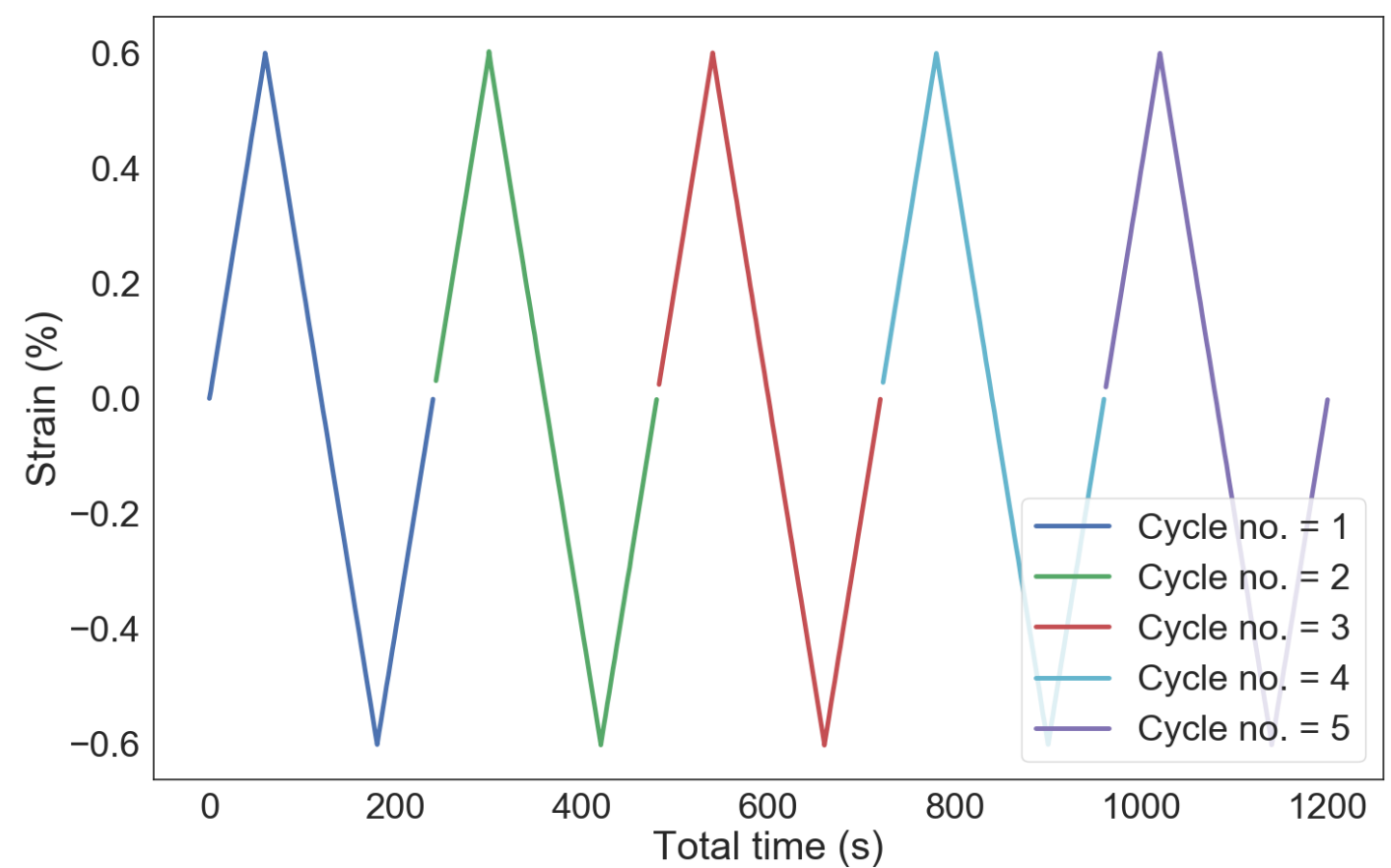

Figure 7. 10 Example first 5 cycles ET-F59 input strain loading.

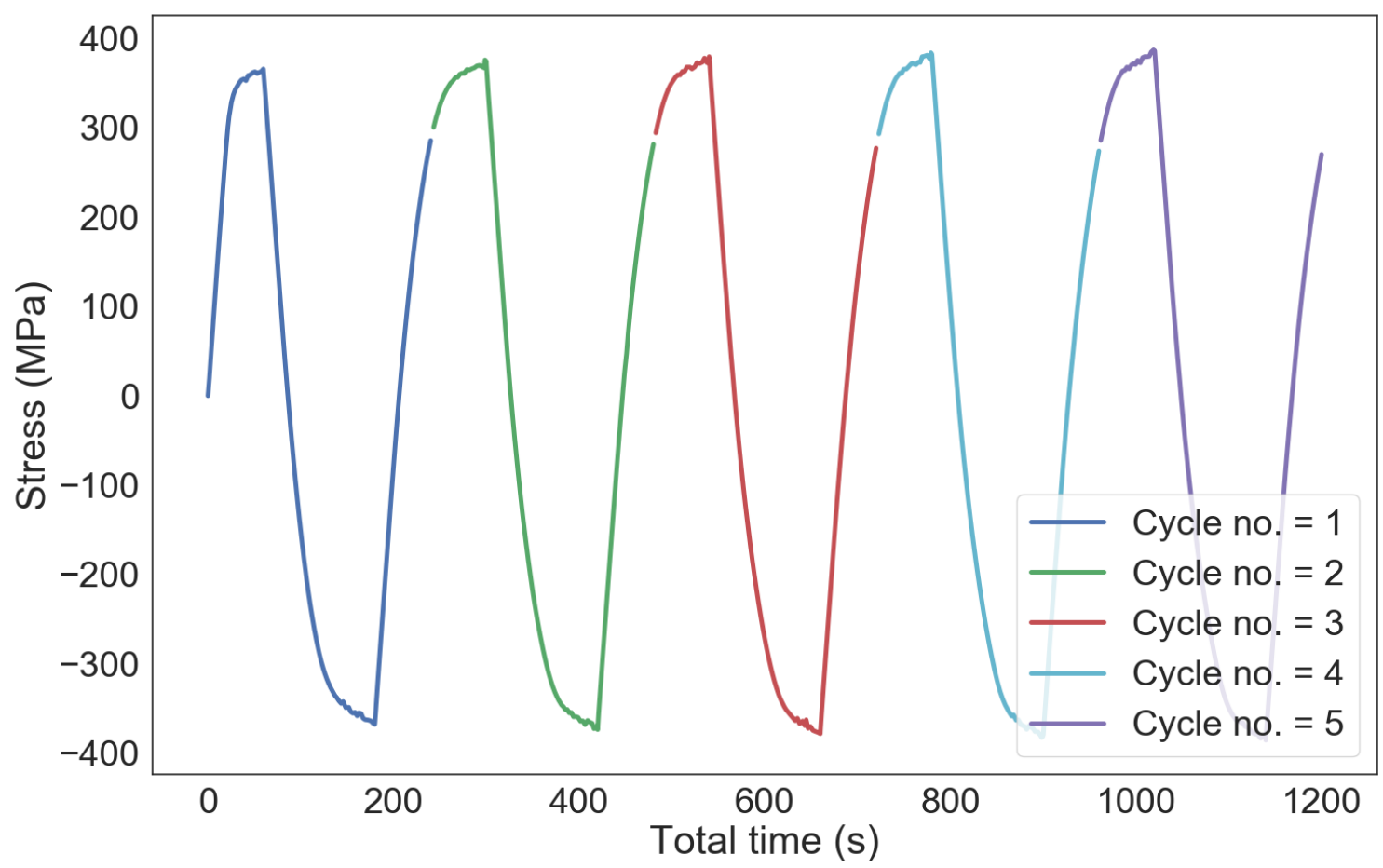

Figure 7. 11 Example first 5 cycles ET-F59 observed stress. 


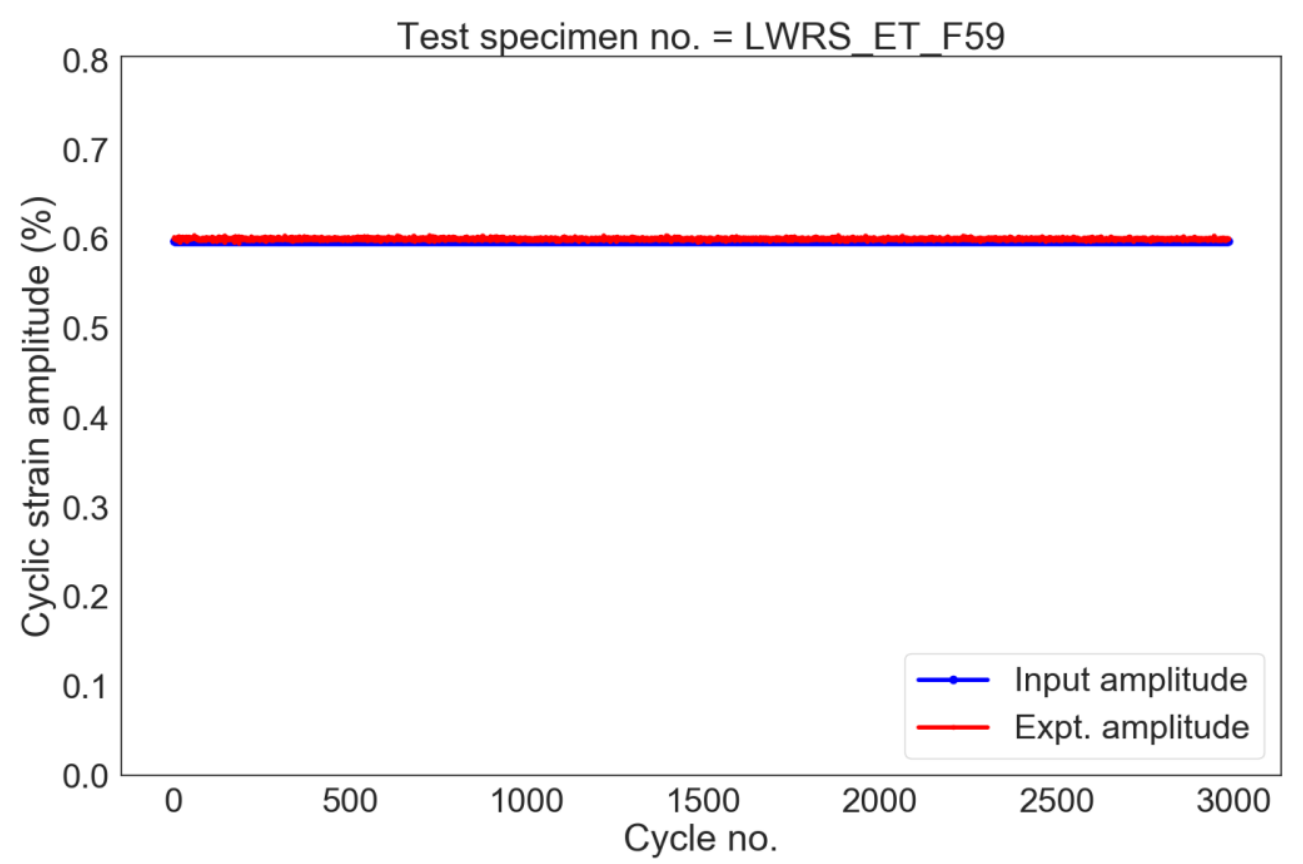

Figure 7. 12 Cycle no. versus maximum cyclic strain amplitude (both applied to analytical model and experimentally observed during the fatigue test of ET-F59 specimen) to track any anomaly in strain loading.

\subsubsection{Cyclic stress analysis results for ET-F59 specimen using fixed tensile properties}

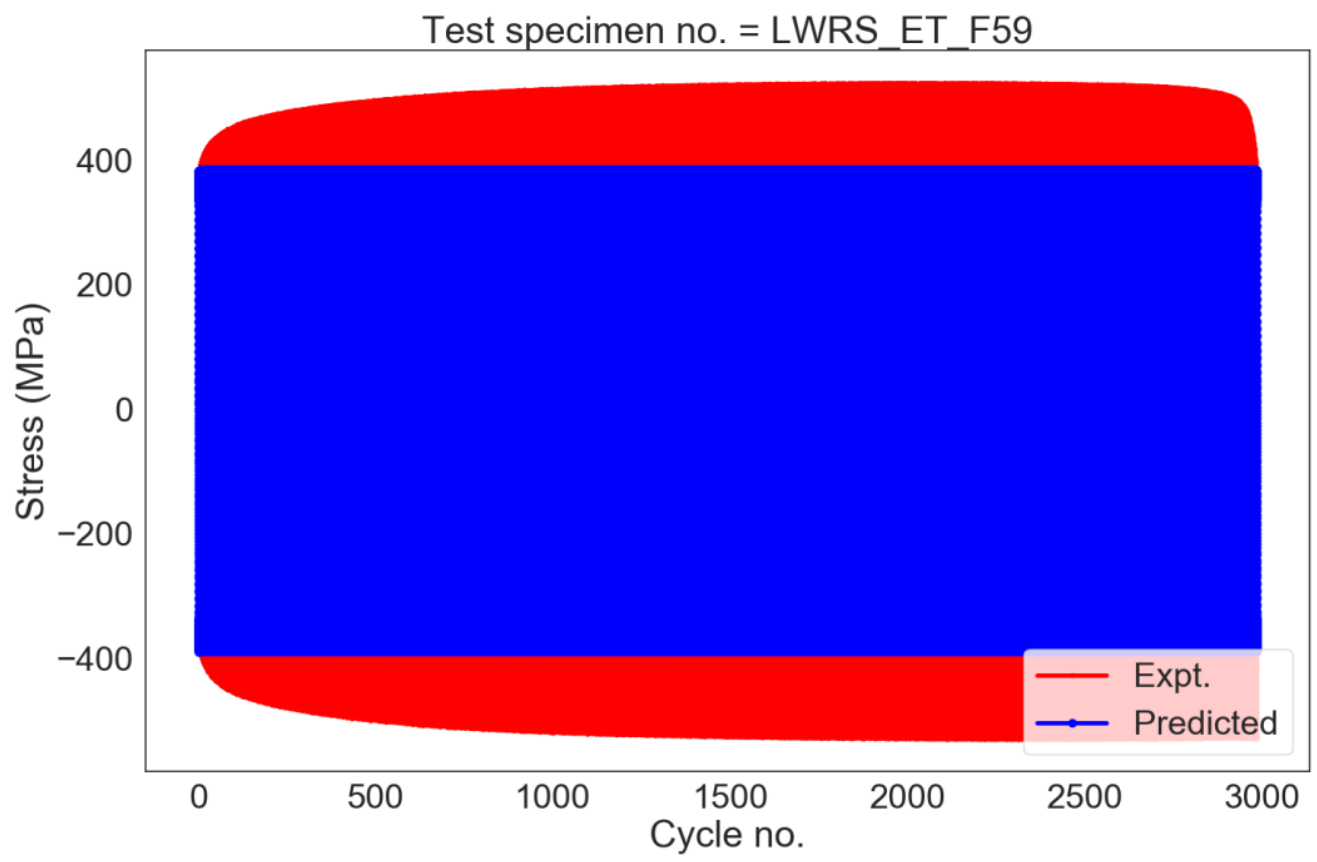

Figure 7. 13 Fixed tensile properties based predicted versus experimentally observed cyclic stress for the entire fatigue life of ET-F59 test case. 


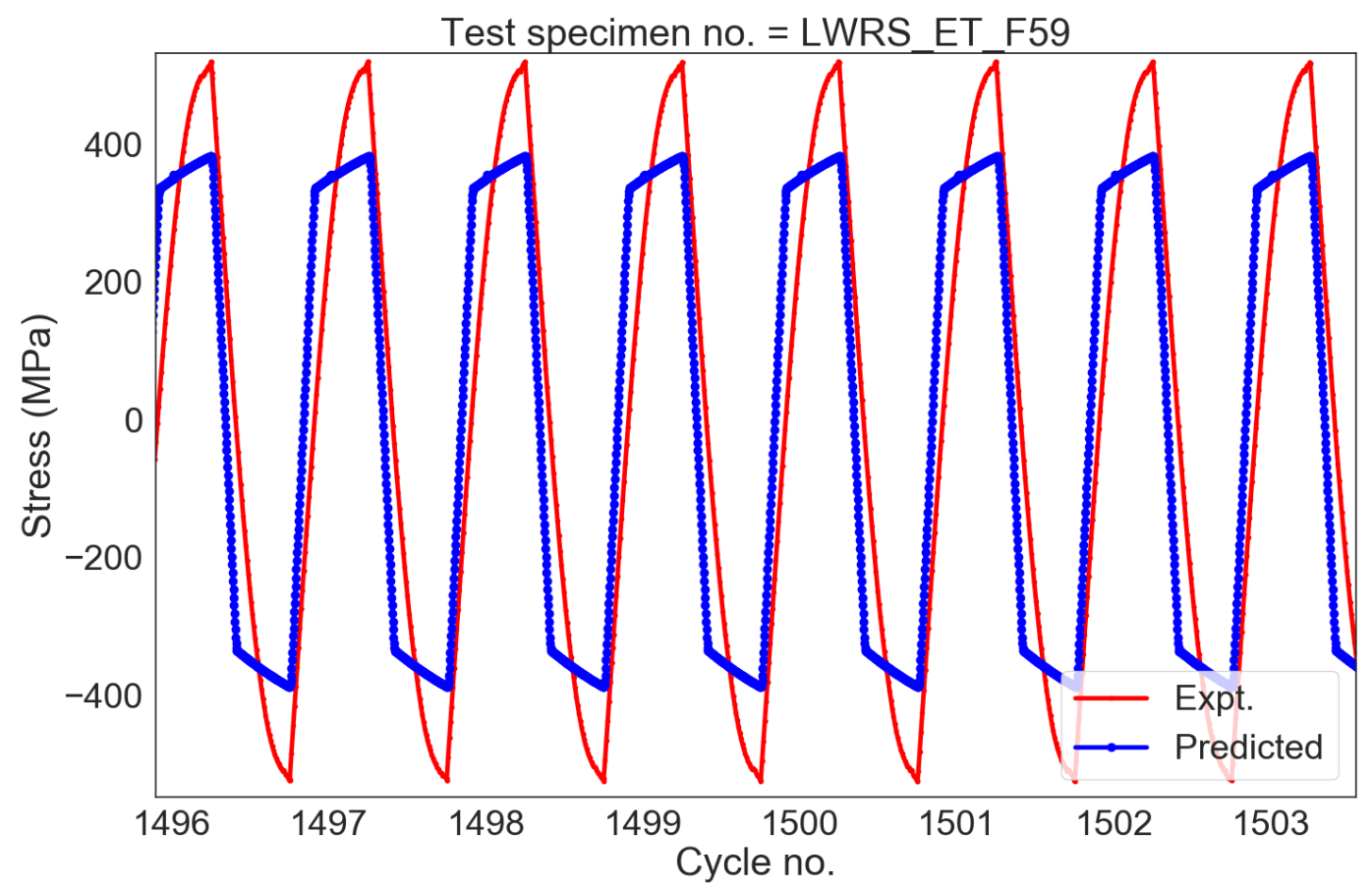

Figure 7. 14 Magnified version of Figure 7.13, approximately during the half-life of ET-F59 specimen.

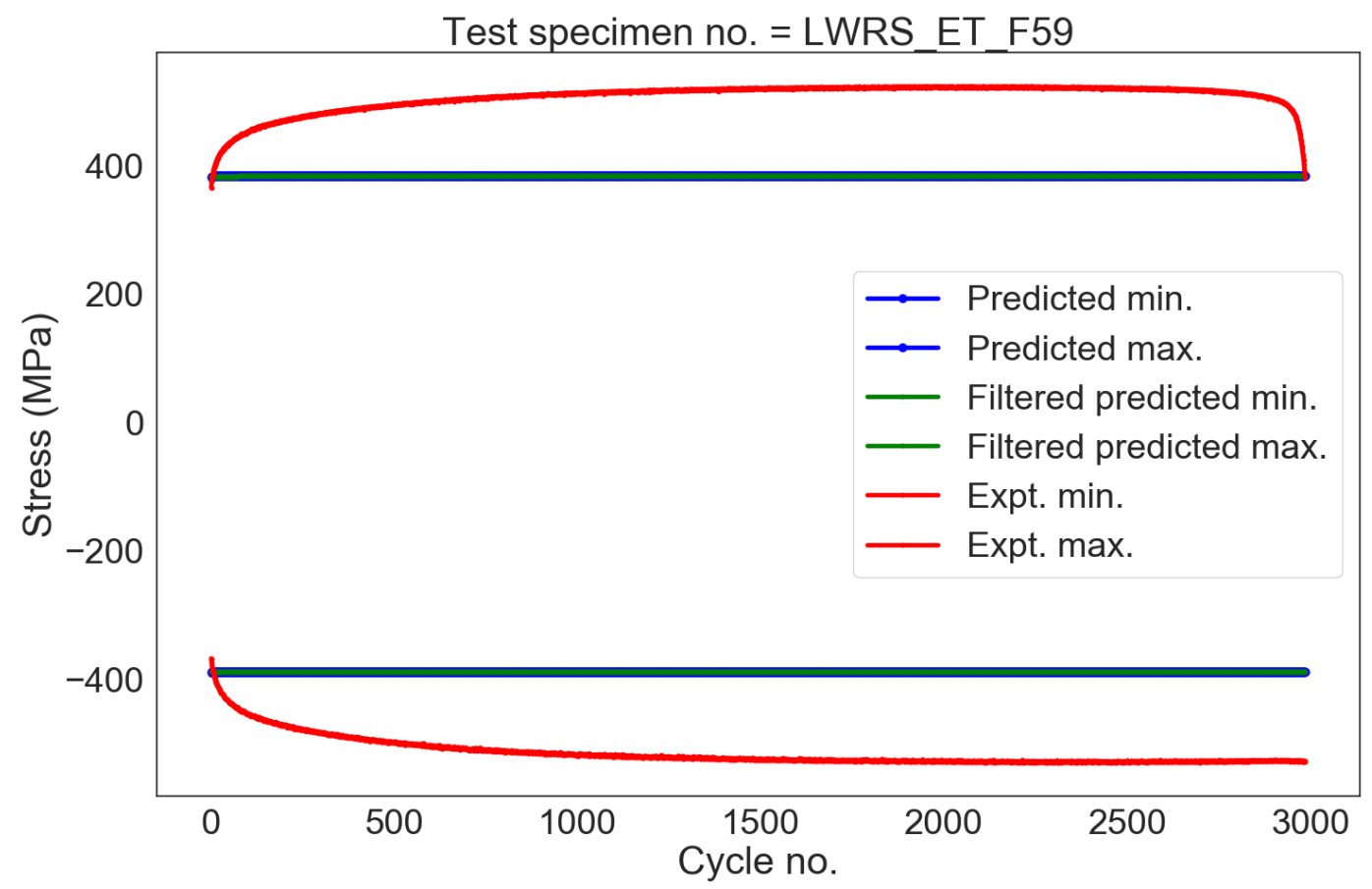

Figure 7. 15 Fixed tensile properties based predicted versus experimentally observed max./min stressamplitudes for the entire fatigue life of ET-F59 test case. 
7.2.2 Cyclic stress analysis results for ET-F59 specimen using variable scaled-cycle-dependent material model properties

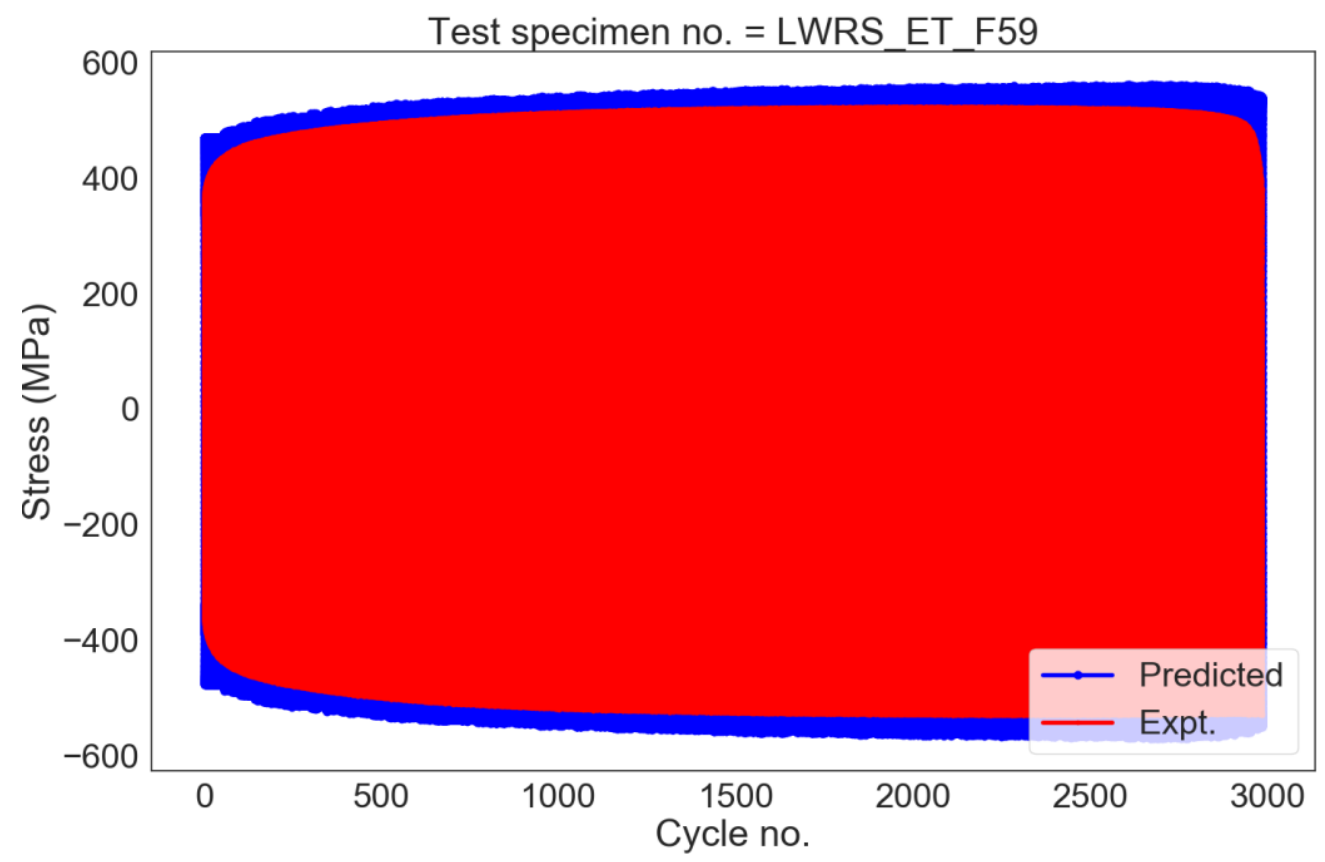

Figure 7. 16 Cycle-dependent properties based predicted versus experimentally observed cyclic stress for the entire fatigue life of ET-F59 test case.

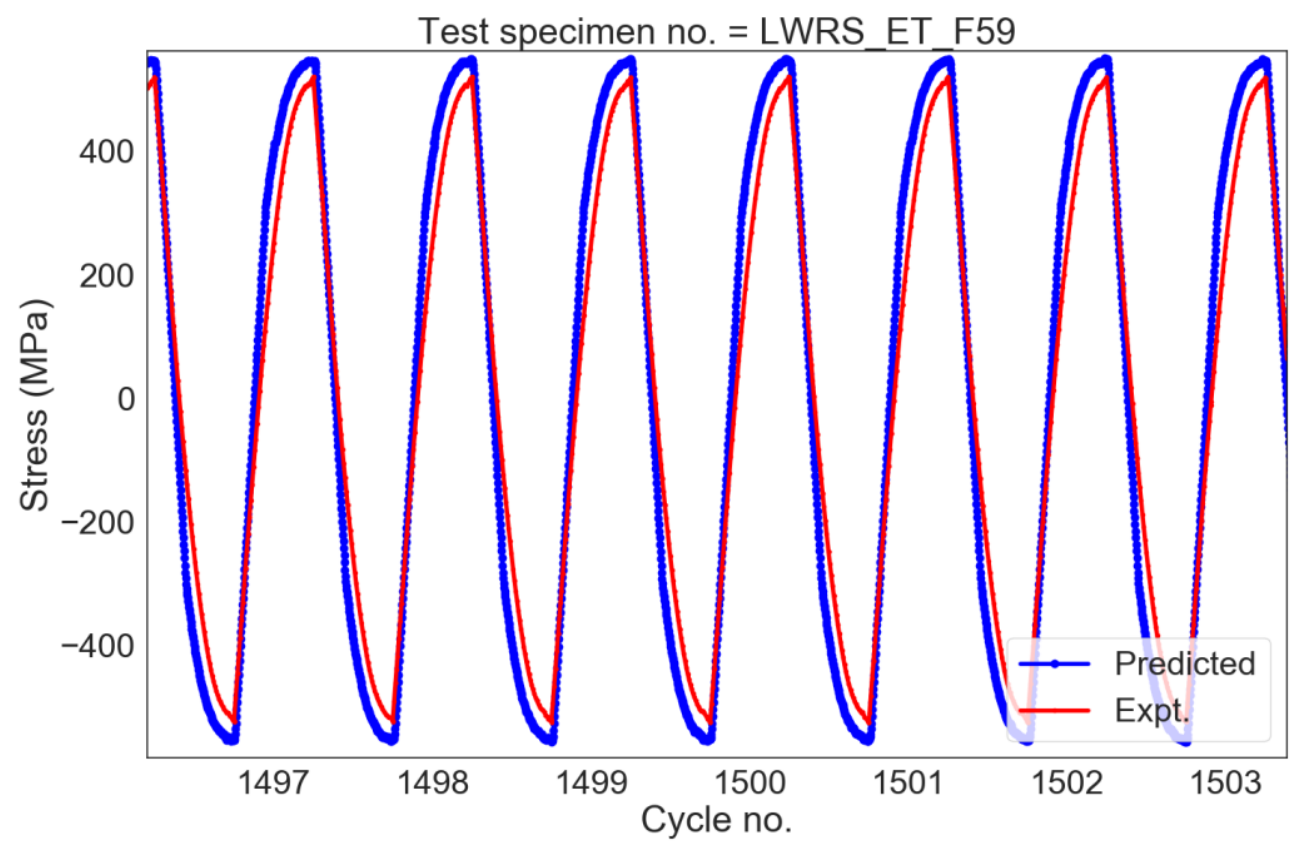

Figure 7. 17 Magnified version of Figure 7.16, approximately during the half-life of ET-F59 specimen. 


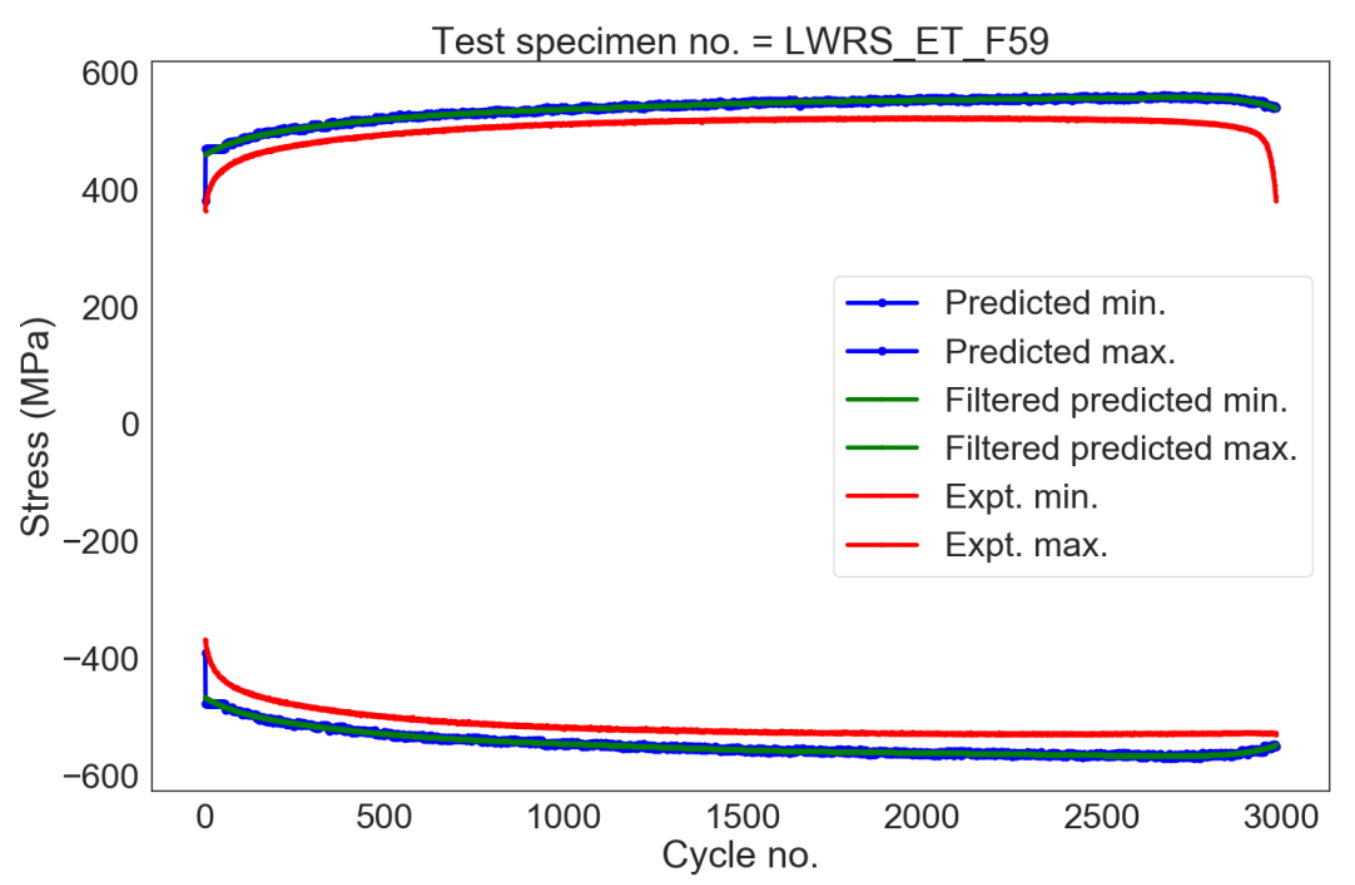

Figure 7. 18 Cycle-dependent properties based predicted versus experimentally observed max./min stress-amplitudes for the entire fatigue life of ET-F59 test case.

\subsection{Cyclic Stress Analysis Results for ET-F60 DMW Specimen Subjected to Pull-rod-control Based Constant Amplitude Displacement Loading}

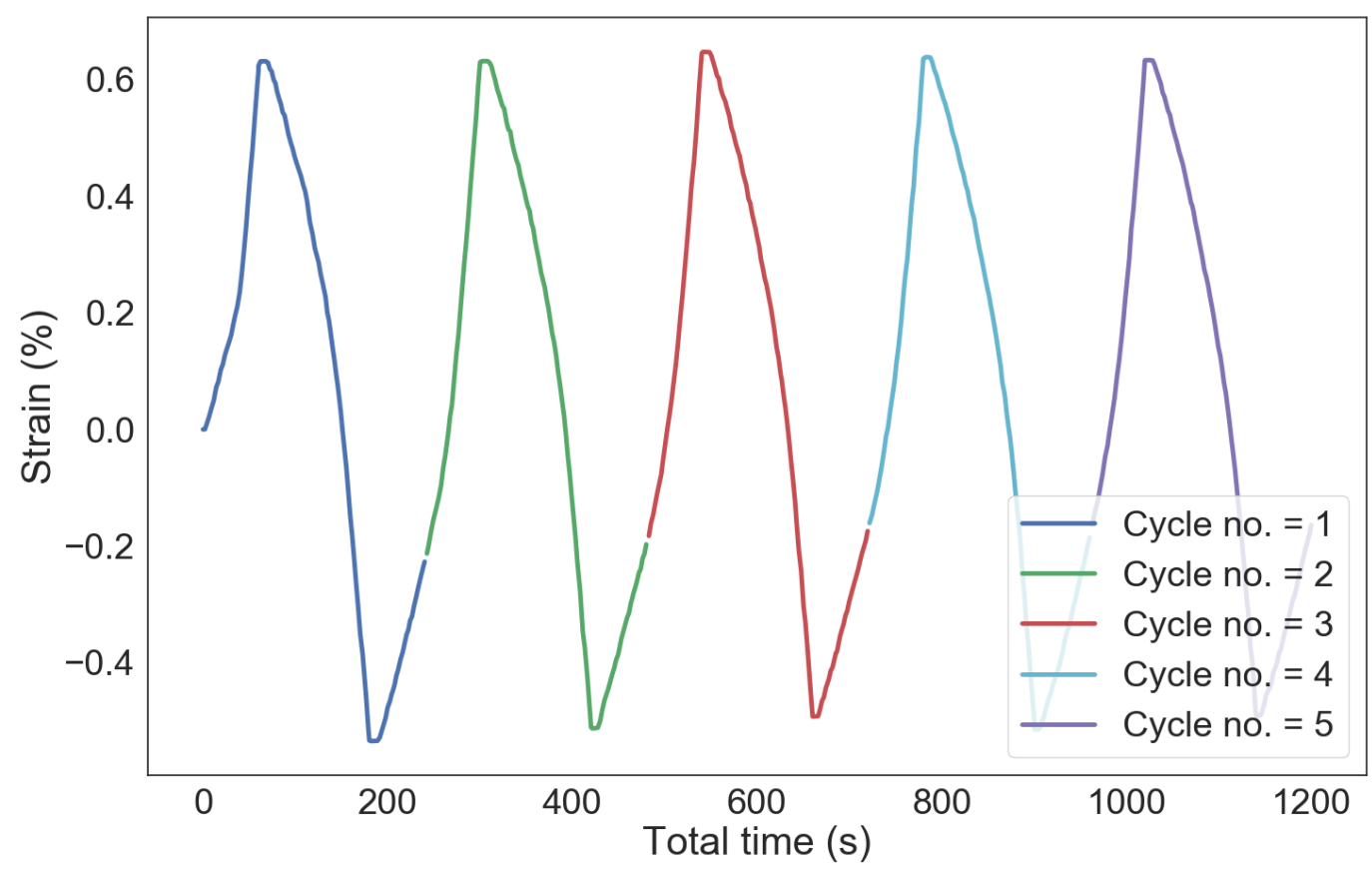

Figure 7. 19 Example first 5 cycles ET-F60 input strain loading. 


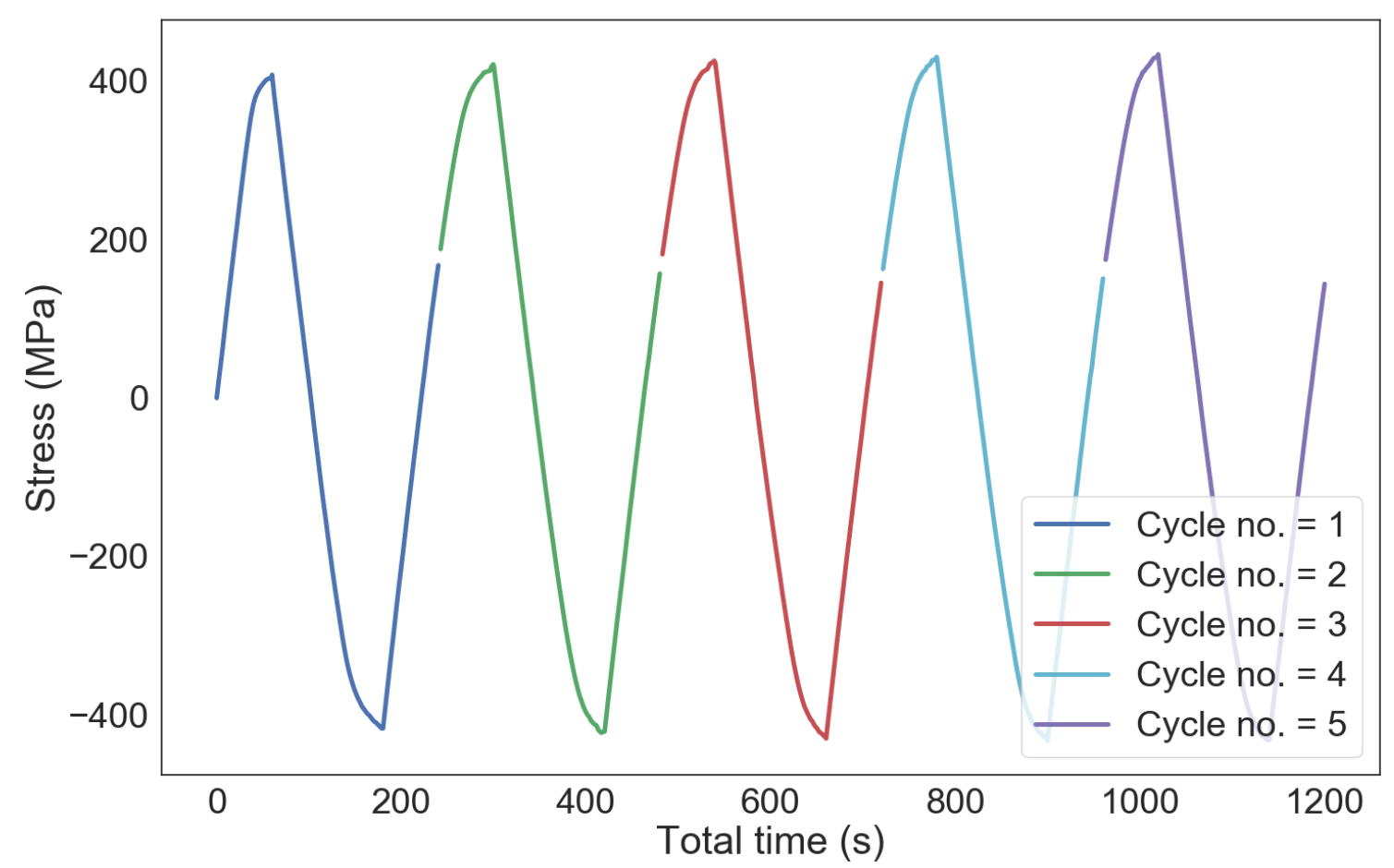

Figure 7. 20 Example first 5 cycles ET-F60 observed stress.

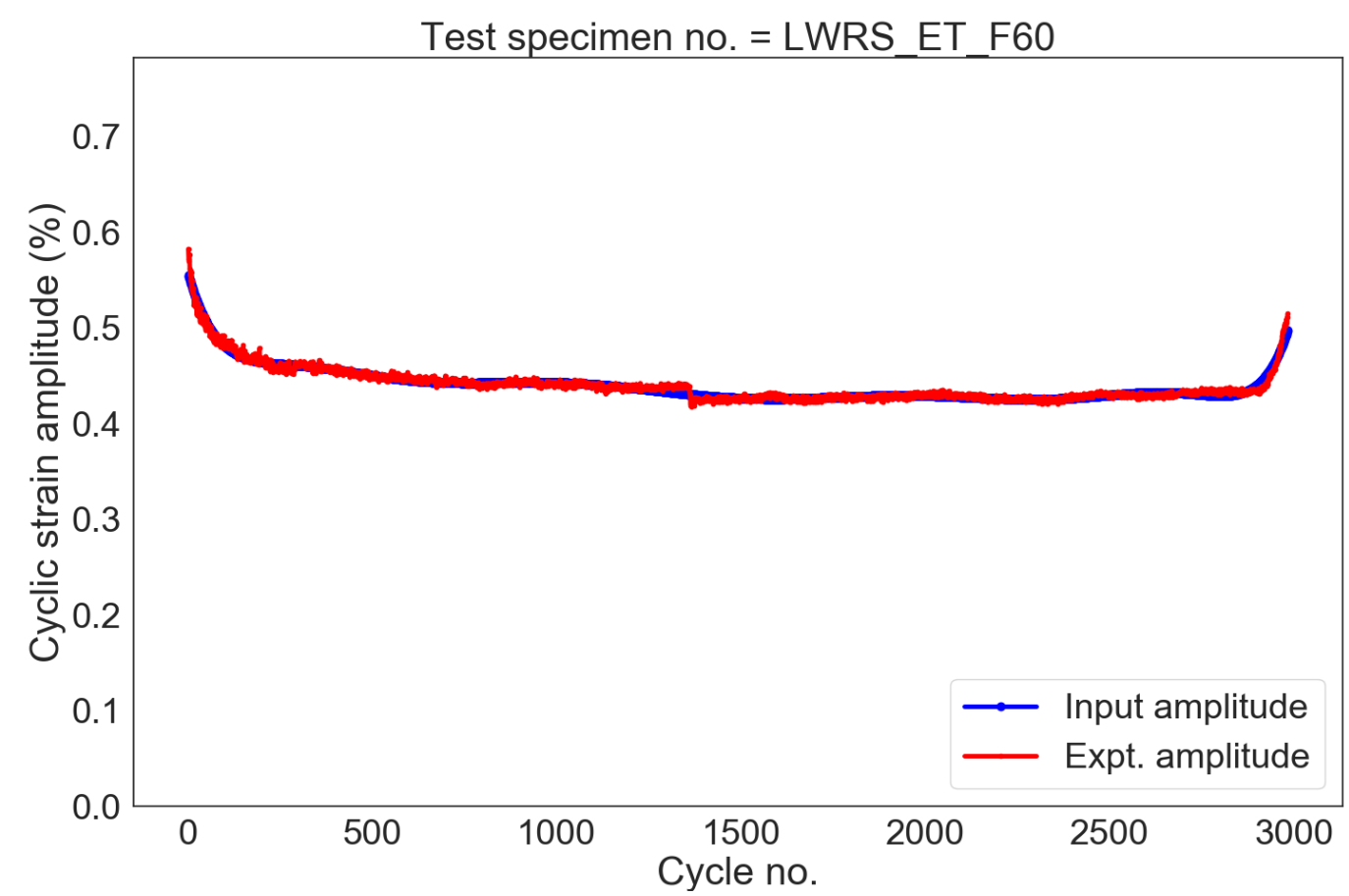

Figure 7. 21 Cycle no. versus maximum strain amplitude (both applied to analytical model and experimentally observed during the fatigue test of ET-F60 specimen) to track any anomaly in strain loading. 
7.3.1 Cyclic stress analysis results for ET-F60 specimen using fixed tensile properties

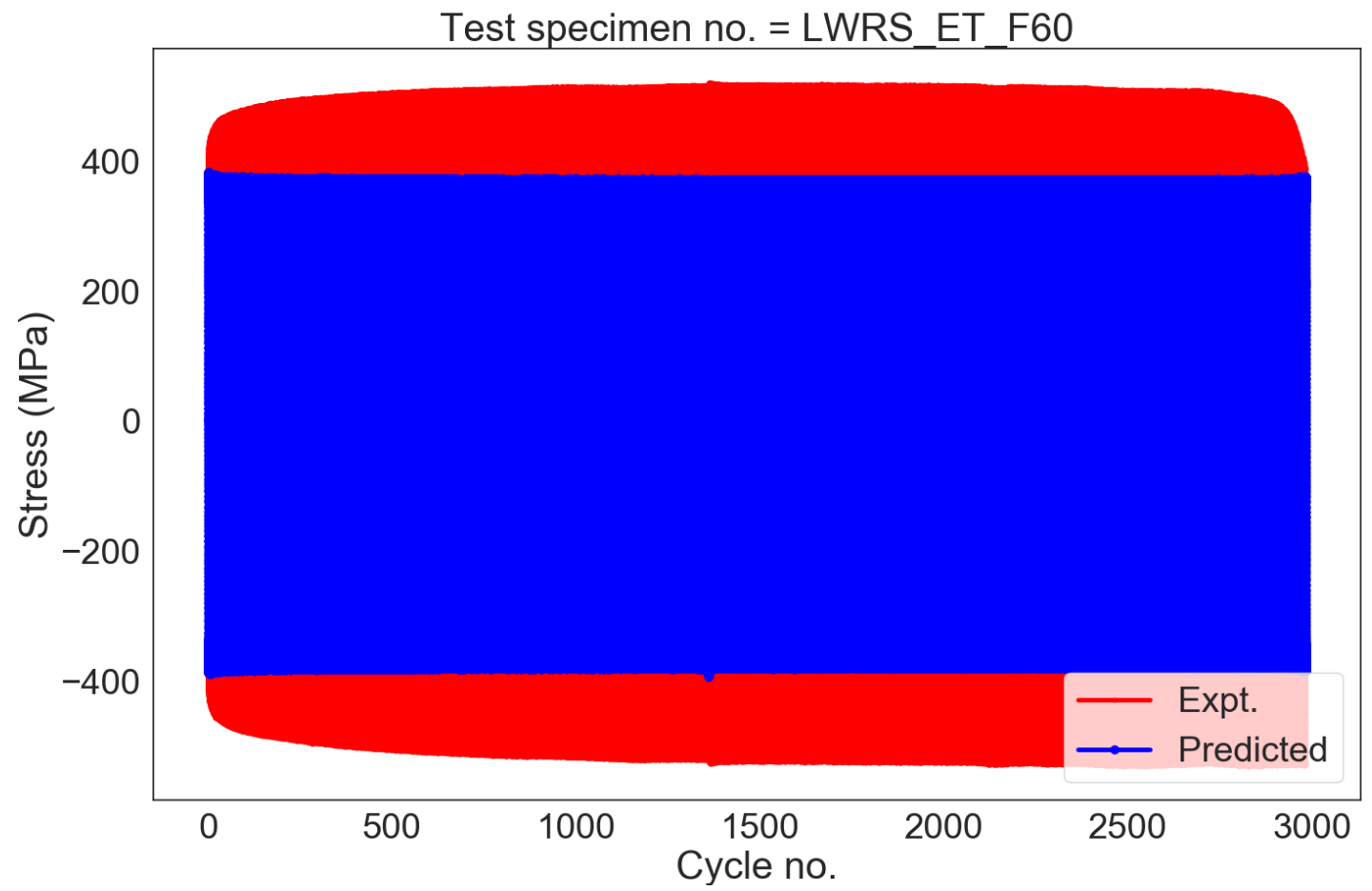

Figure 7. 22 Fixed tensile properties based predicted versus experimentally observed cyclic stress for the entire fatigue life of ET-F60 test case.

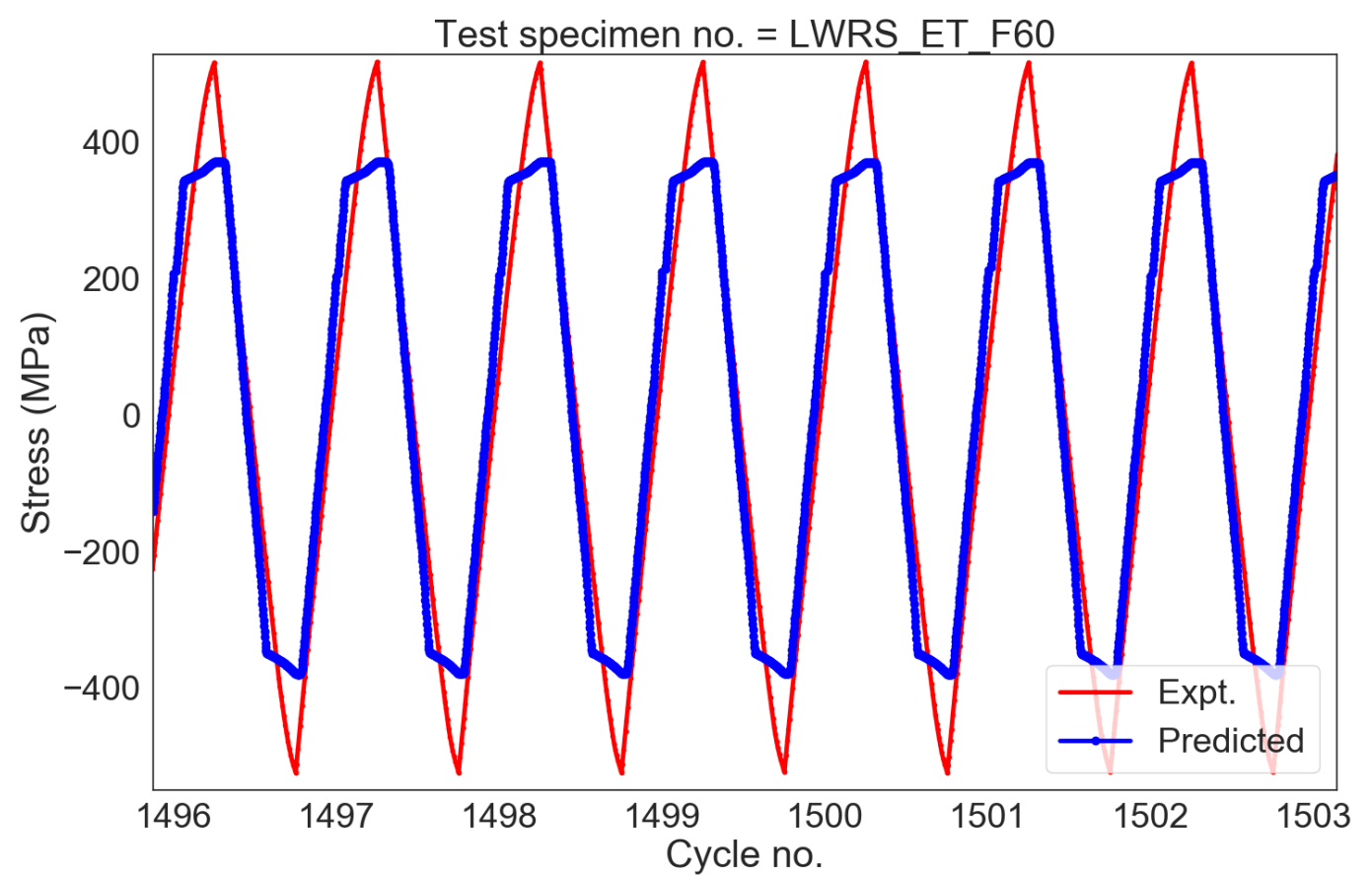

Figure 7. 23 Magnified version of Figure 7.22, approximately during the half-life of ET-F60 specimen. 


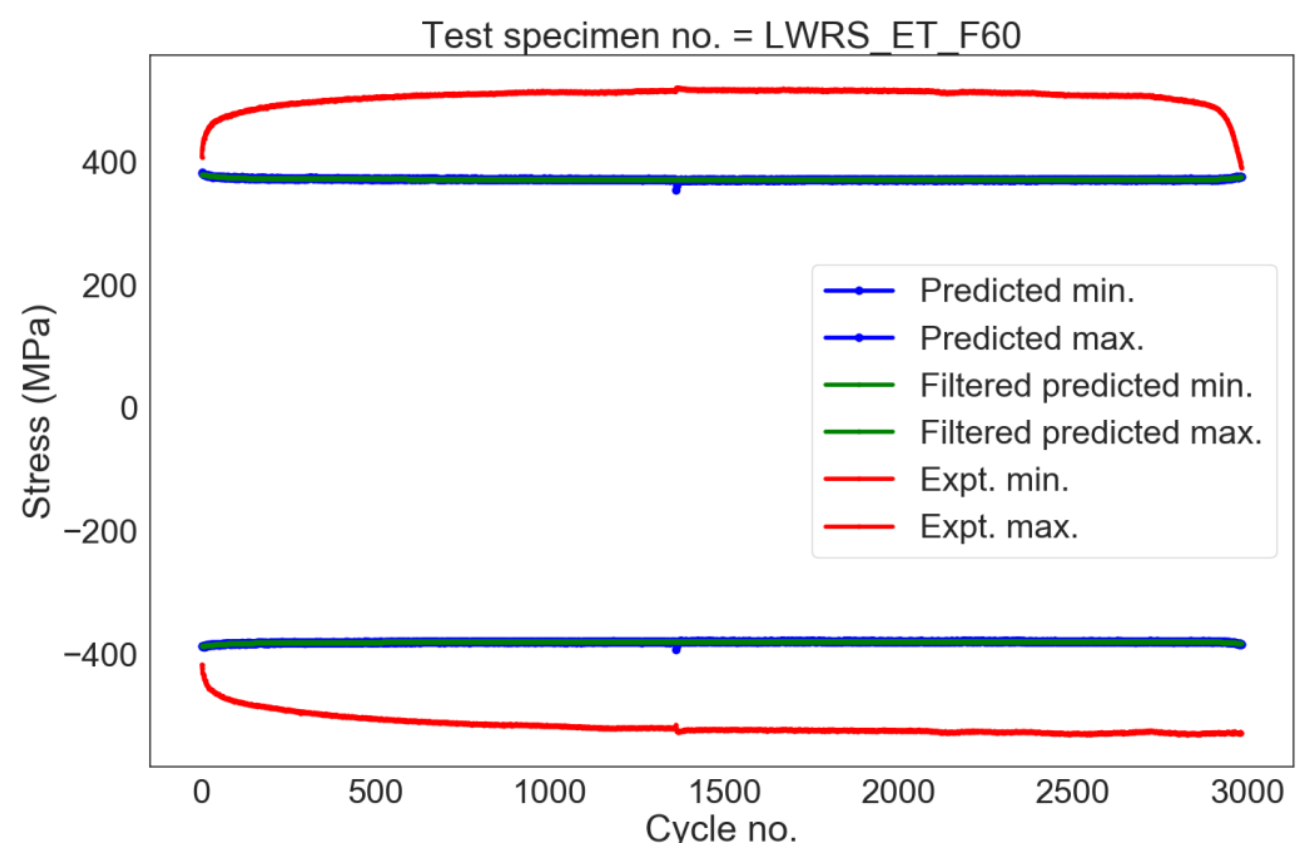

Figure 7. 24 Fixed tensile properties based predicted versus experimentally observed max./min stressamplitudes for the entire fatigue life of ET-F60 test case.

\subsubsection{Cyclic stress analysis results for ET-F60 specimen using variable scaled-cycle-dependent} material model properties

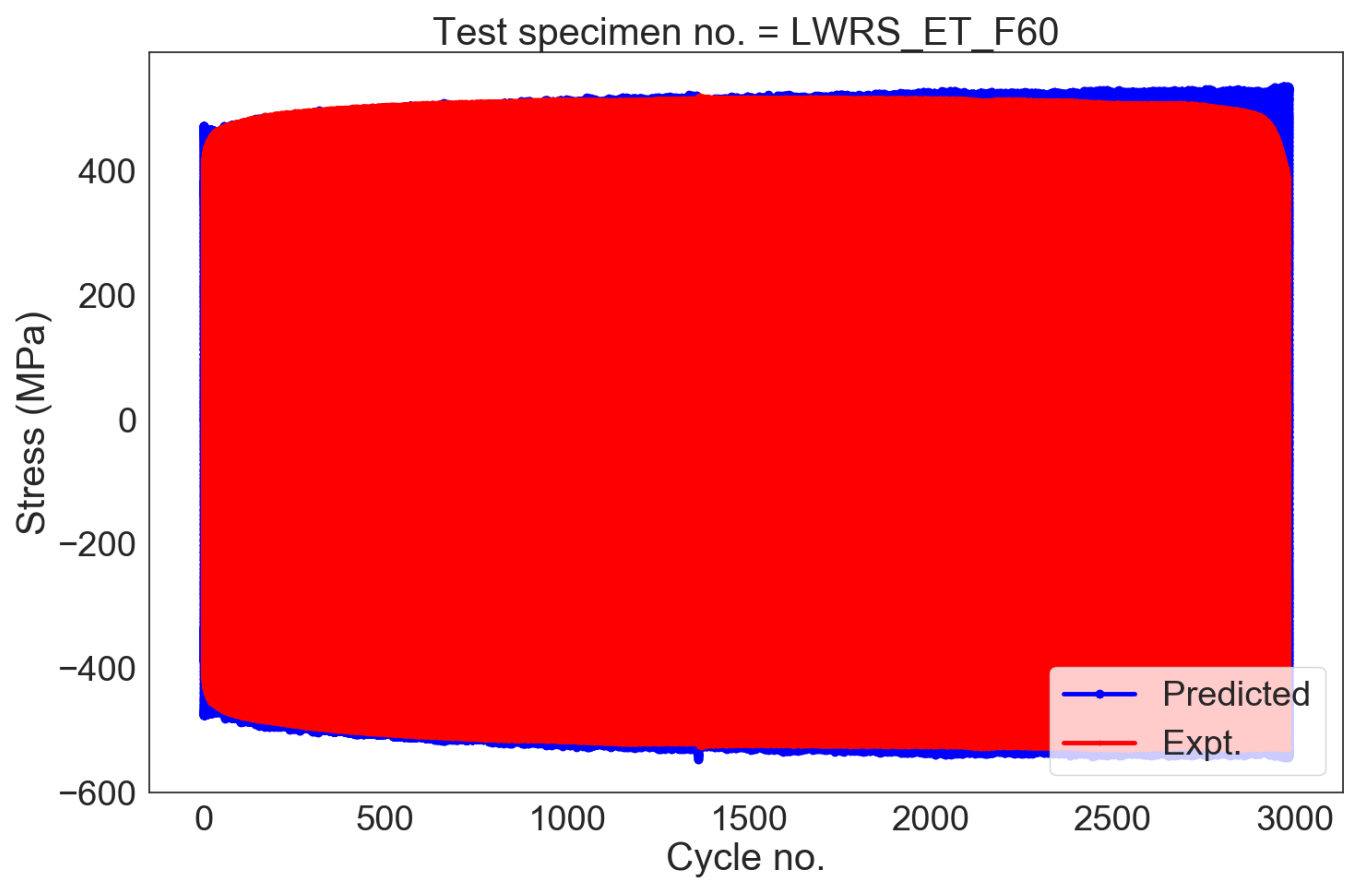

Figure 7. 25 Cycle-dependent properties based predicted versus experimentally observed cyclic stress for the entire fatigue life of ET-F60 test case. 


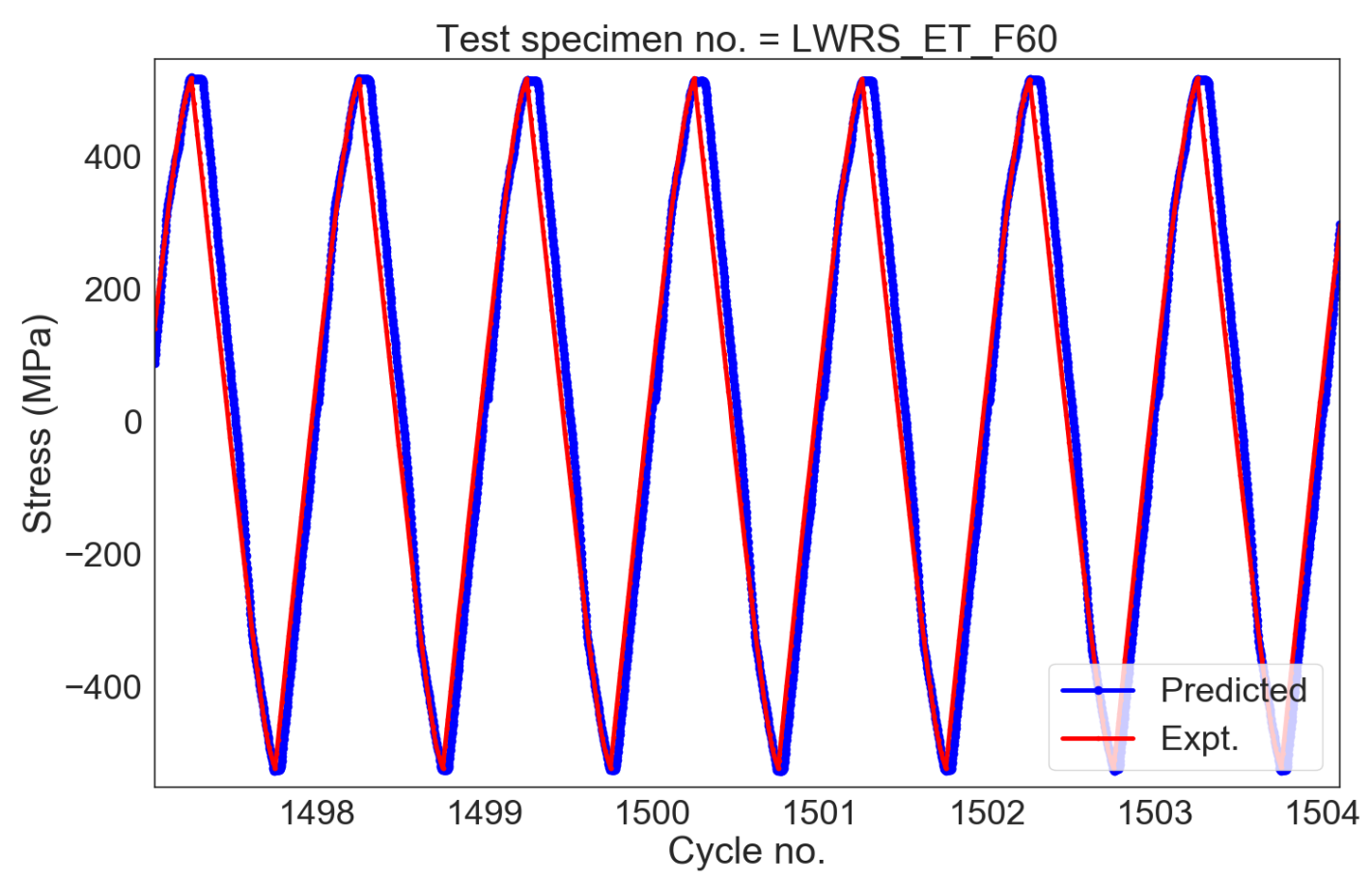

Figure 7. 26 Magnified version of Figure 7.25, approximately during the half-life of ET-F60 specimen.

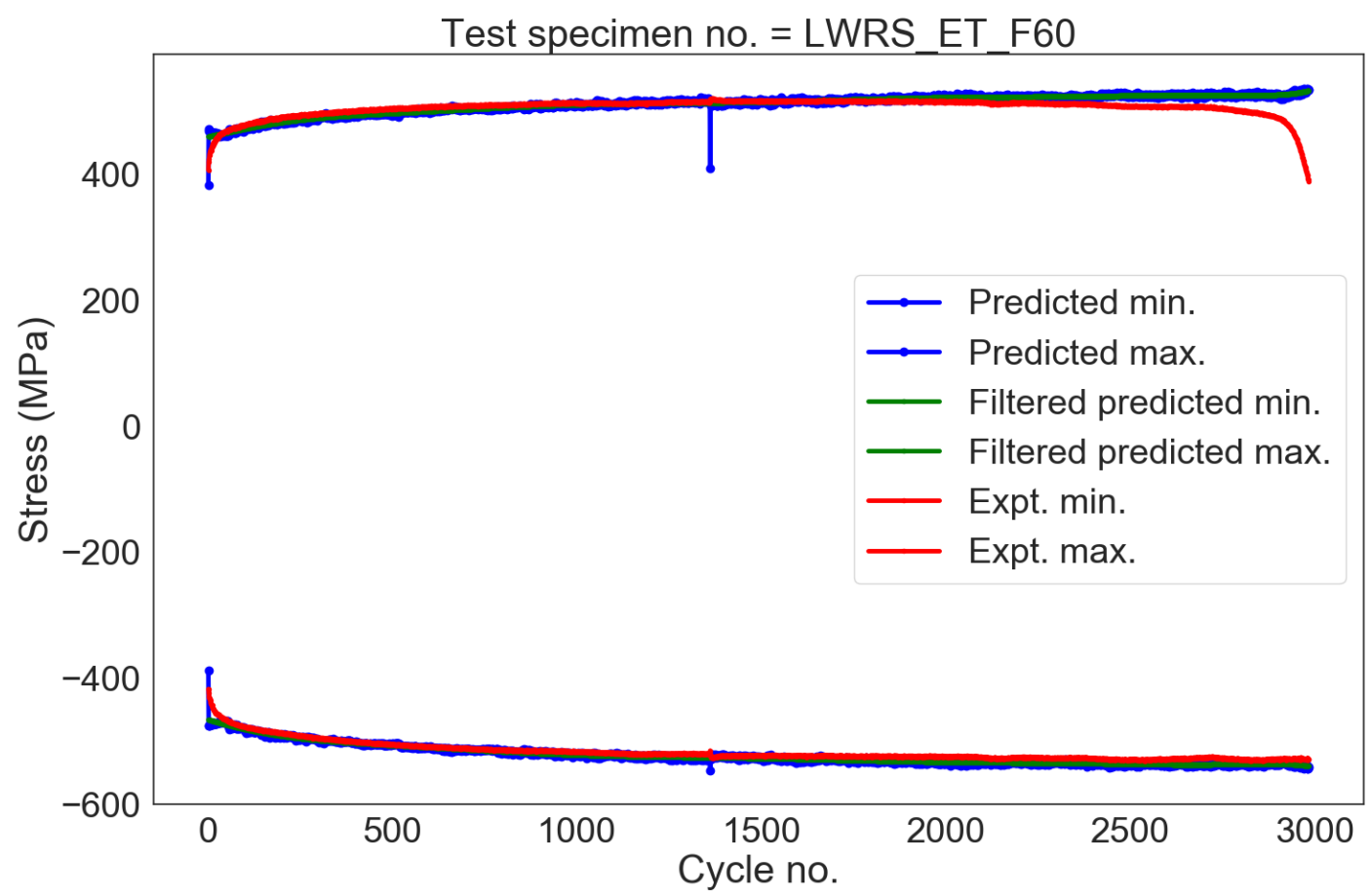

Figure 7.27 Cycle-dependent properties based predicted versus experimentally observed max./min stress-amplitudes for the entire fatigue life of ET-F60 test case. 
7.4 Cyclic Stress Analysis Results for ET-F61 DMW Specimen Subjected to AI-Guided and Pull-rodcontrol Based Variable Amplitude Displacement Loading

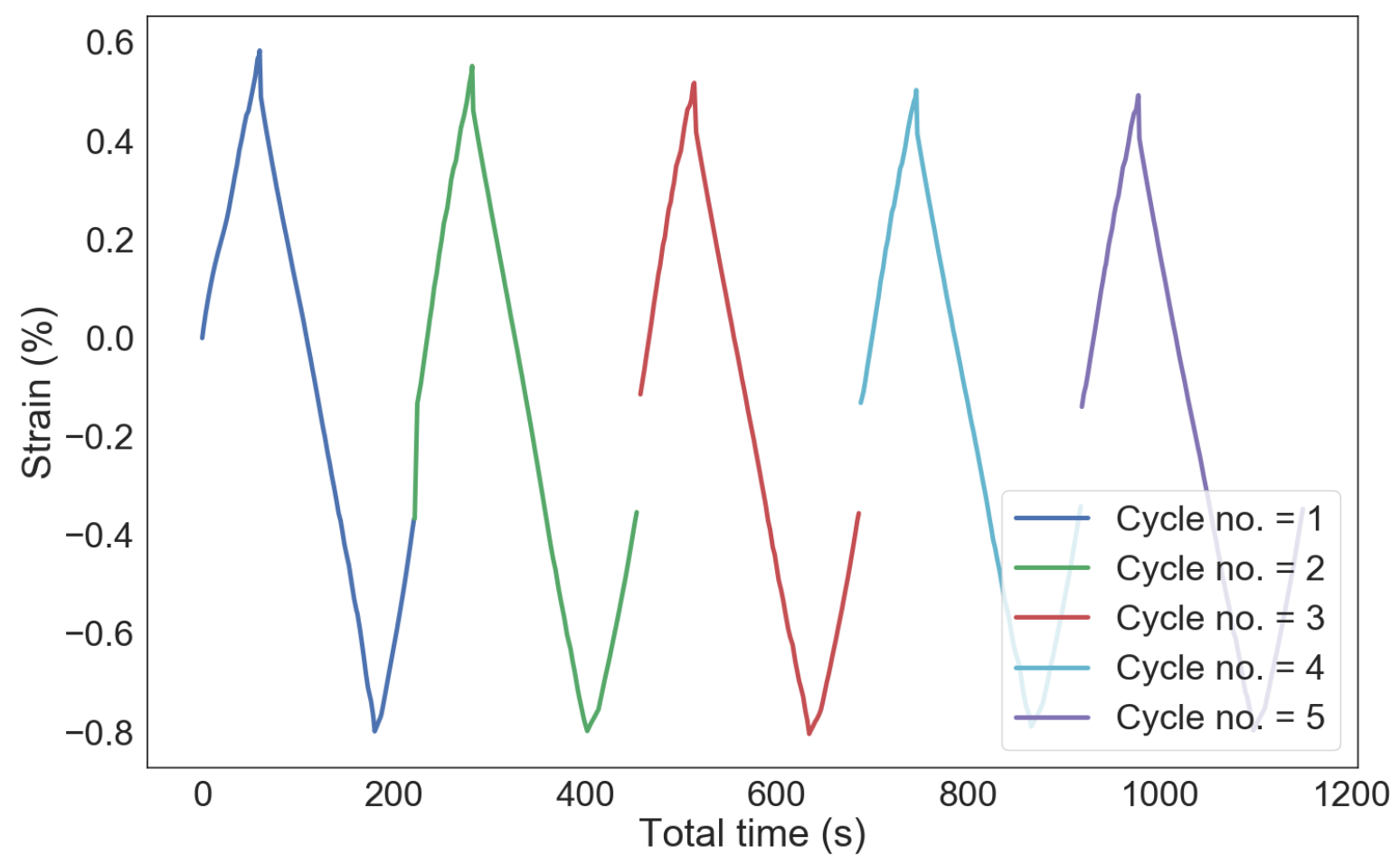

Figure 7. 28 Example first 5 cycles ET-F61 input strain loading.

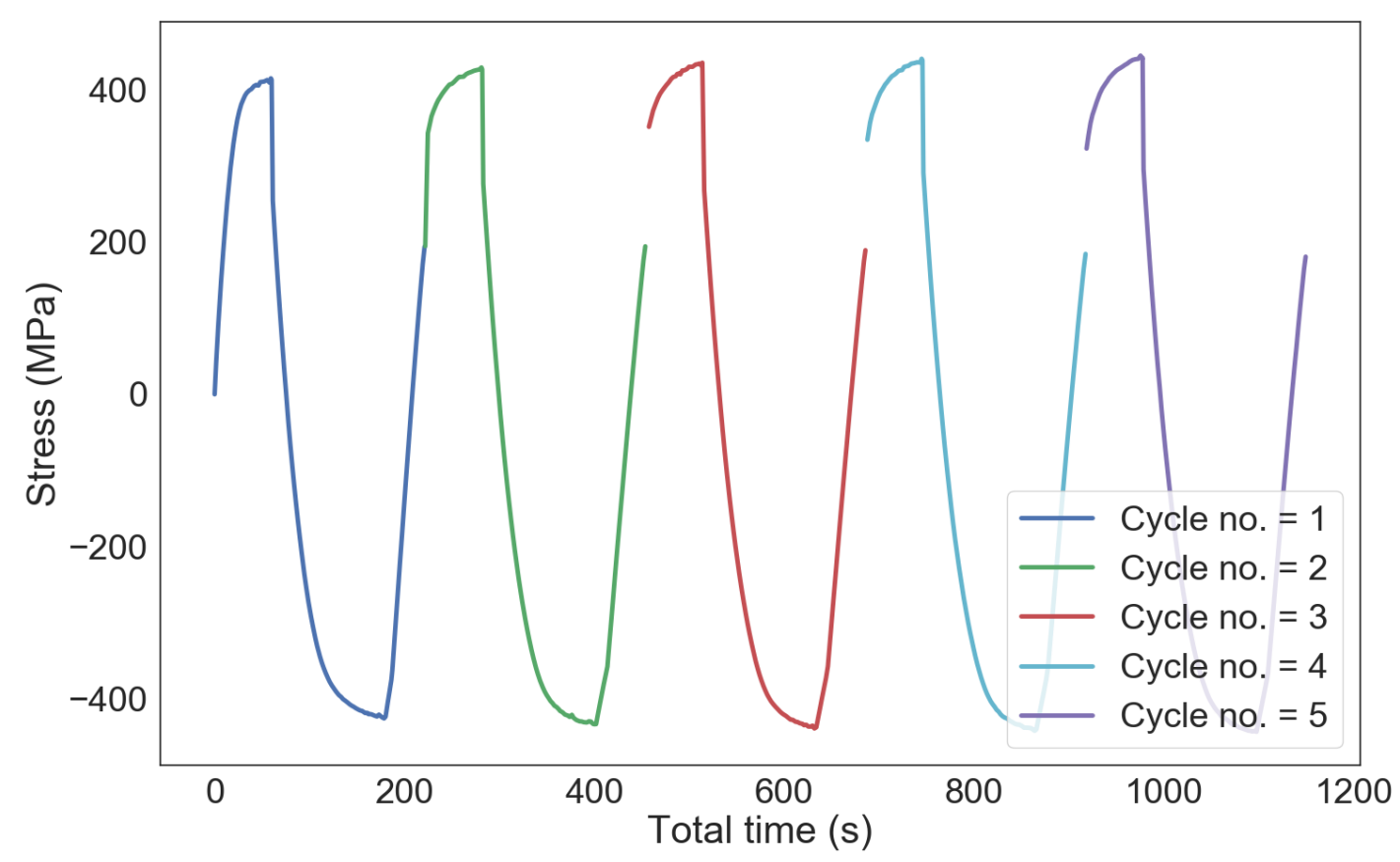

Figure 7. 29 Example first 5 cycles ET-F61 observed stress. 


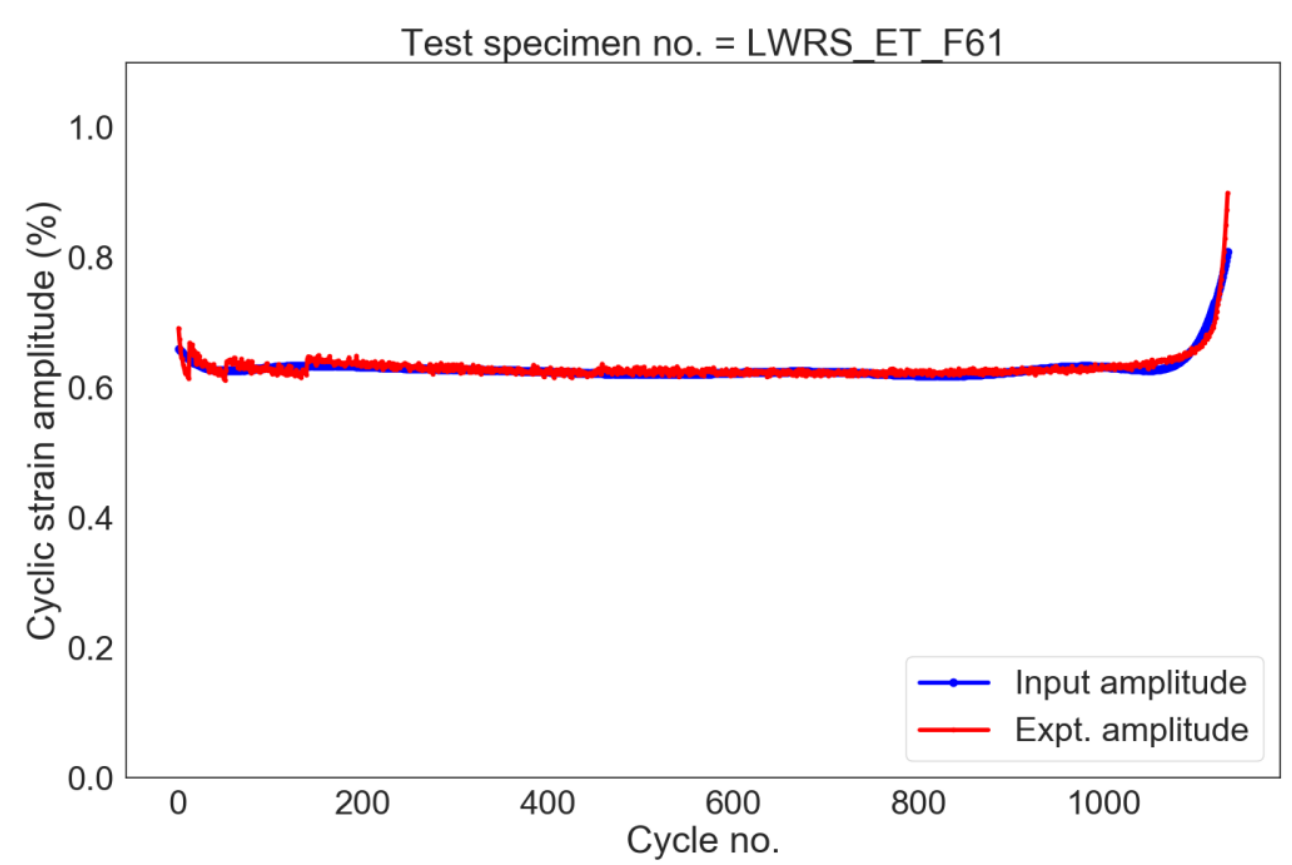

Figure 7. 30 Cycle no. versus maximum cyclic strain amplitude (both applied to analytical model and experimentally observed during the fatigue test of ET-F61 specimen) to track any anomaly in strain loading.

\subsubsection{Cyclic stress analysis results for ET-F61 specimen using fixed tensile properties}

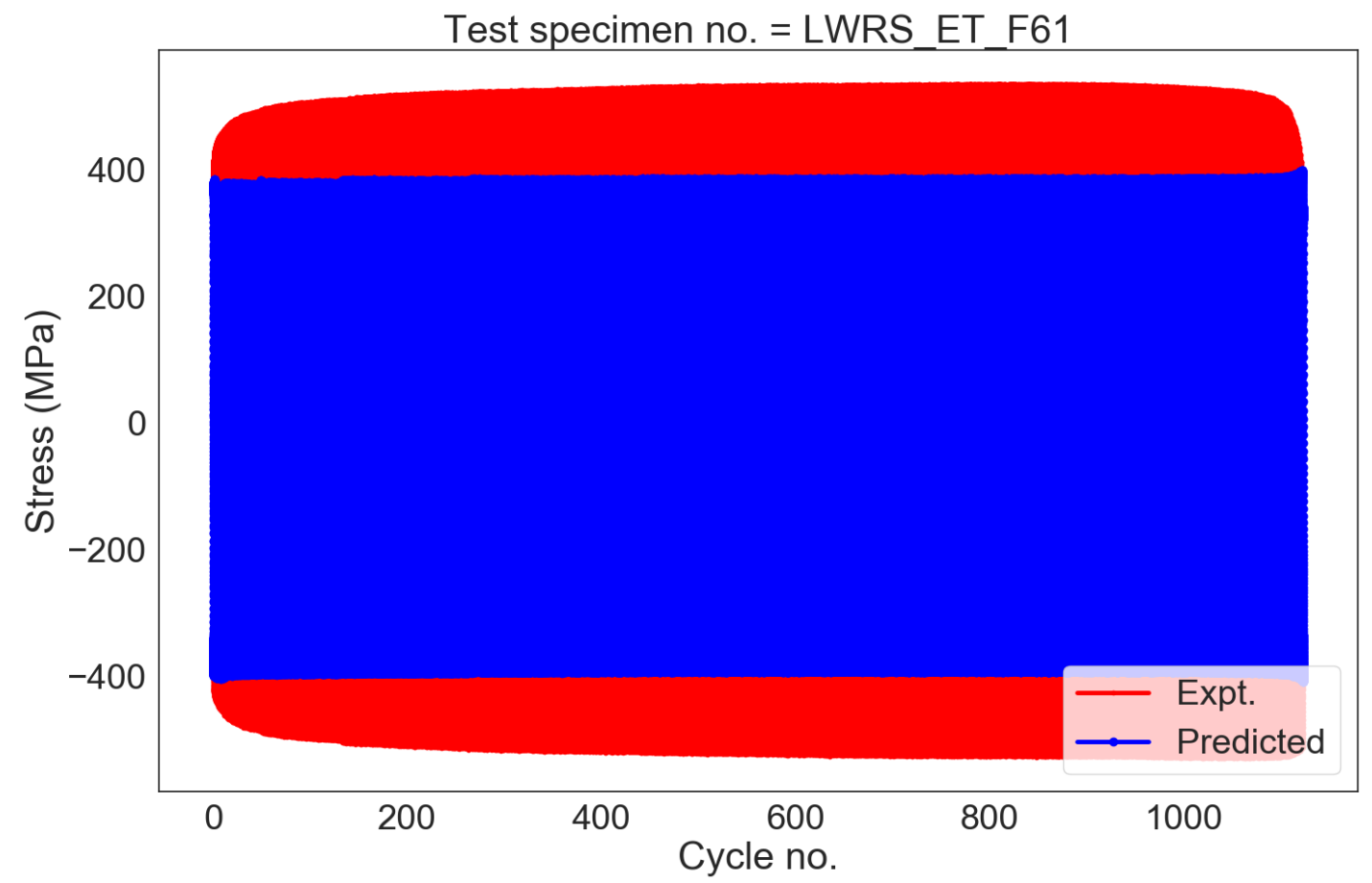

Figure 7. 31 Fixed tensile properties based predicted versus experimentally observed cyclic stress for the entire fatigue life of ET-F61 test case. 


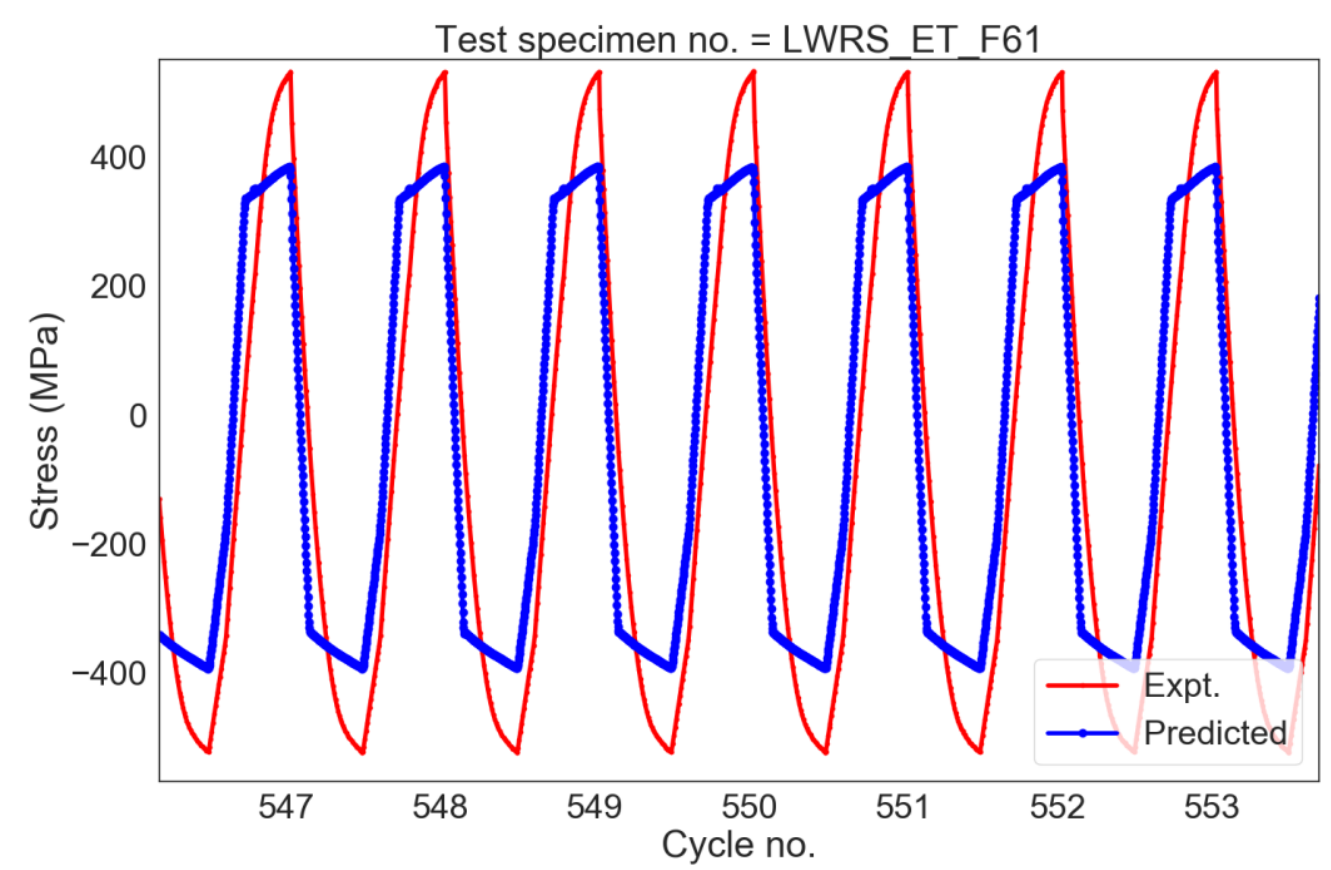

Figure 7. 32 Magnified version of Figure 7.31, approximately during the half-life of ET-F61 specimen.

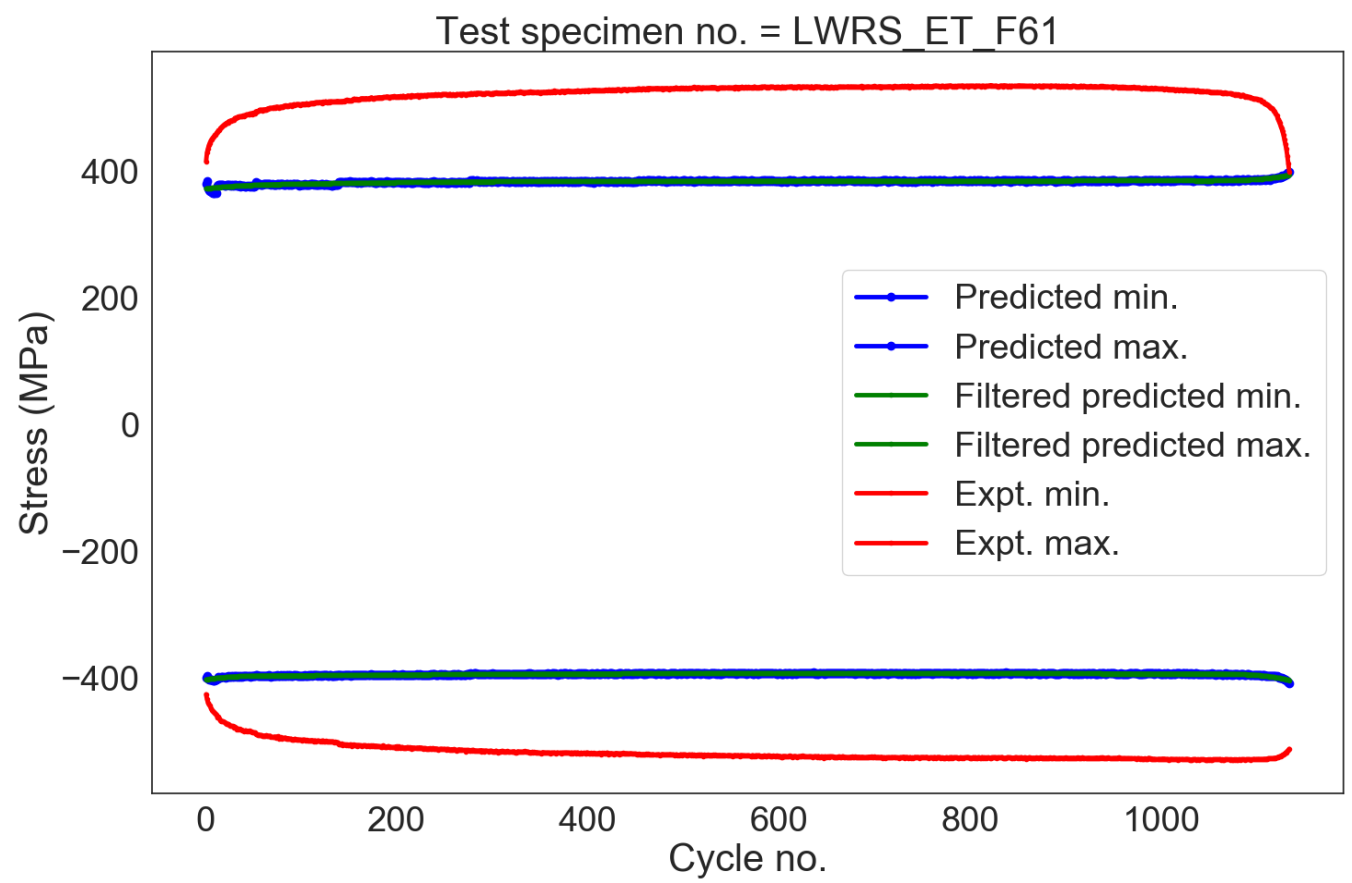

Figure 7. 33 Fixed tensile properties based predicted versus experimentally observed max./min stressamplitudes for the entire fatigue life of ET-F61 test case. 
7.4.2 Cyclic stress analysis results for ET-F61 specimen using variable cyclic-plasticity material model properties

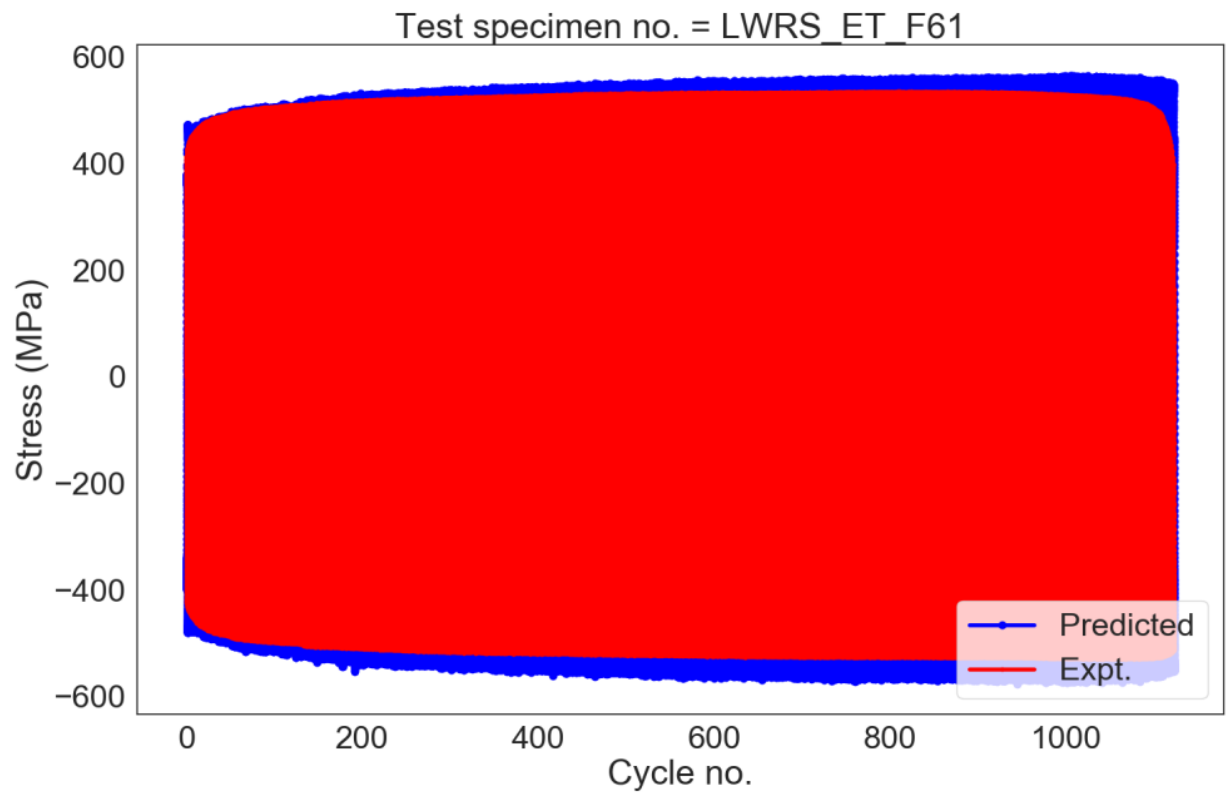

Figure 7. 34 Cycle-dependent properties based predicted versus experimentally observed cyclic stress for the entire fatigue life of ET-F61 test case.

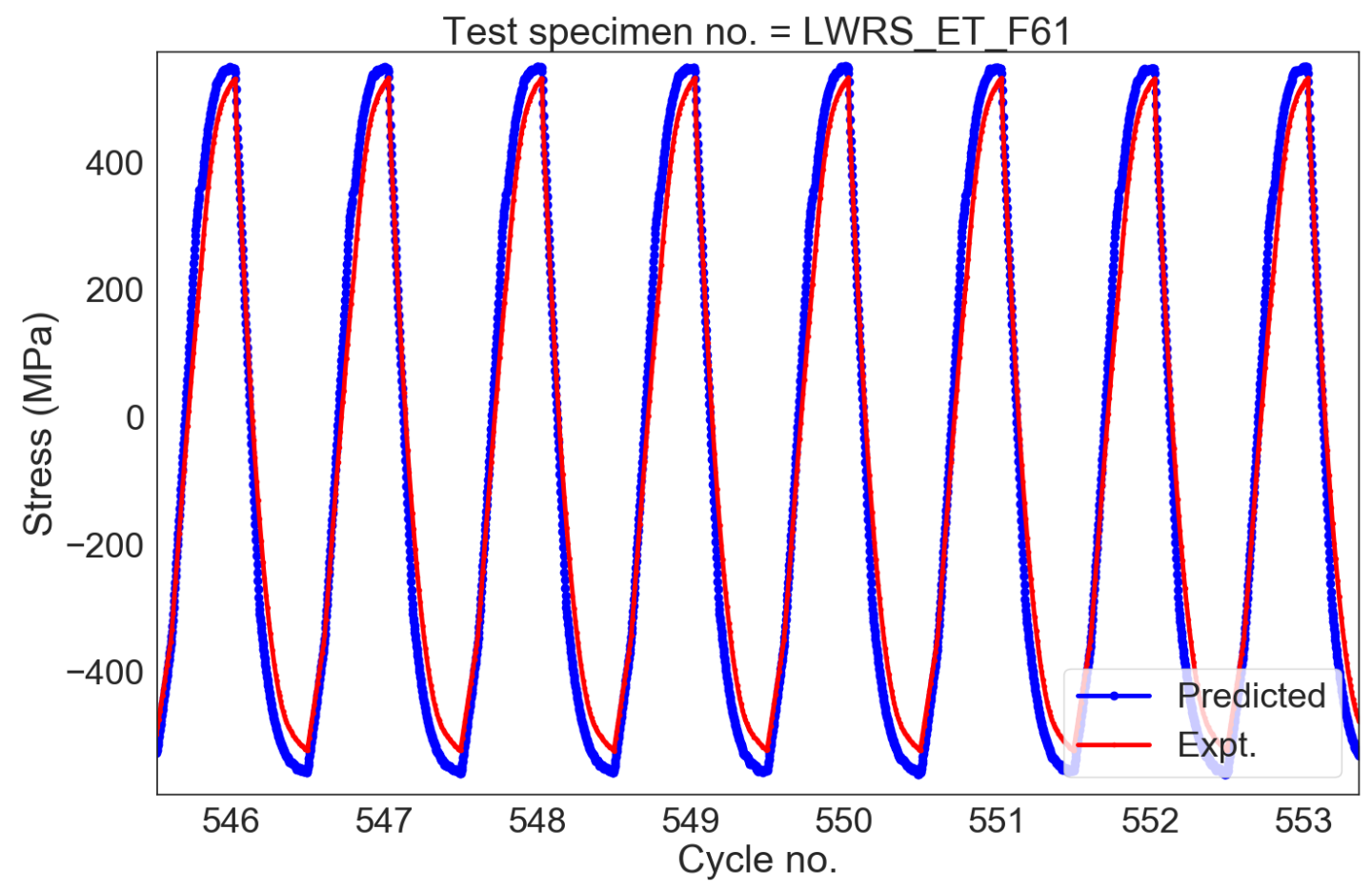

Figure 7. 35 Magnified version of Figure 7.34, approximately during the half-life of ET-F61 specimen. 


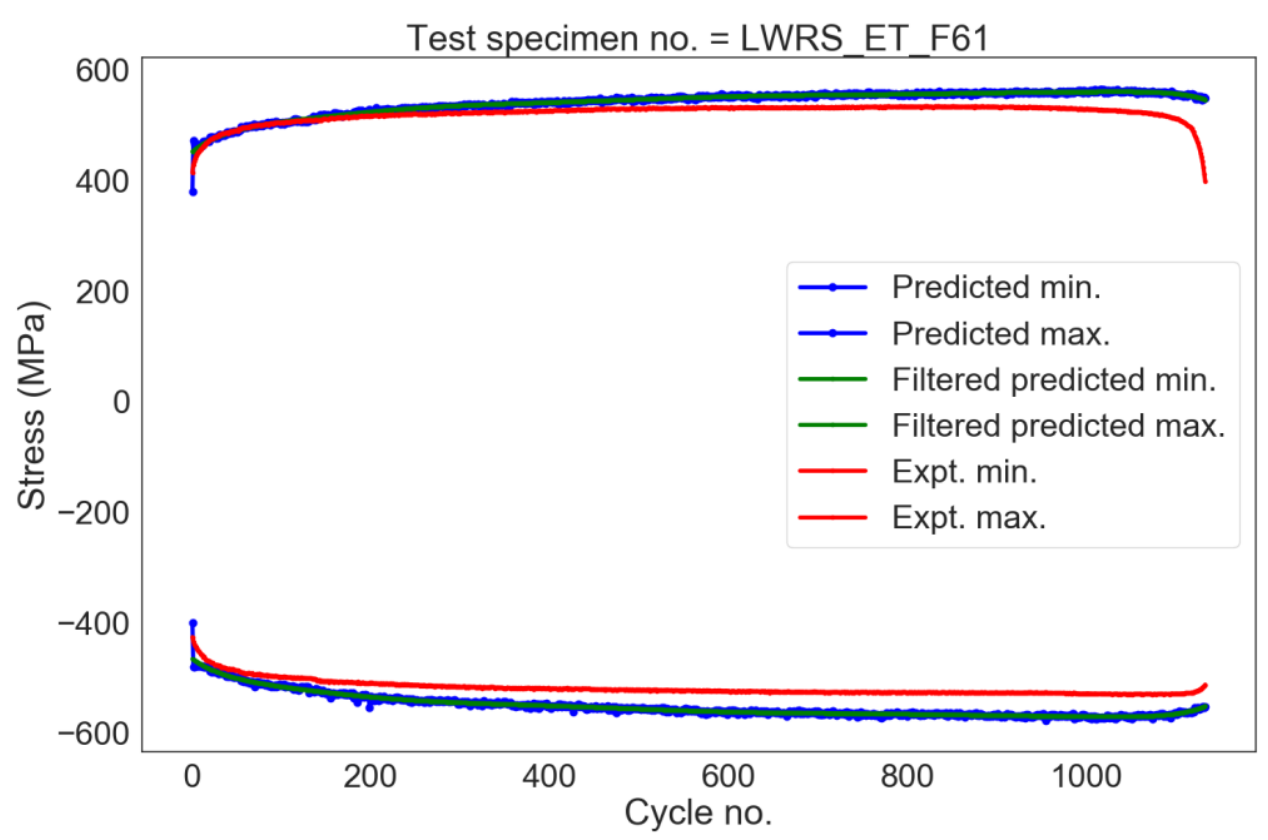

Figure 7. 36 Cycle-dependent properties based predicted versus experimentally observed max./min stress-amplitudes for the entire fatigue life of ET-F61 test case.

\subsection{Cyclic Stress Analysis Results for ET-F57 DMW Specimen Based on Accumulated Strain-energy Dependent Material Models}

\subsubsection{Accumulated strain-energy dependent material properties}

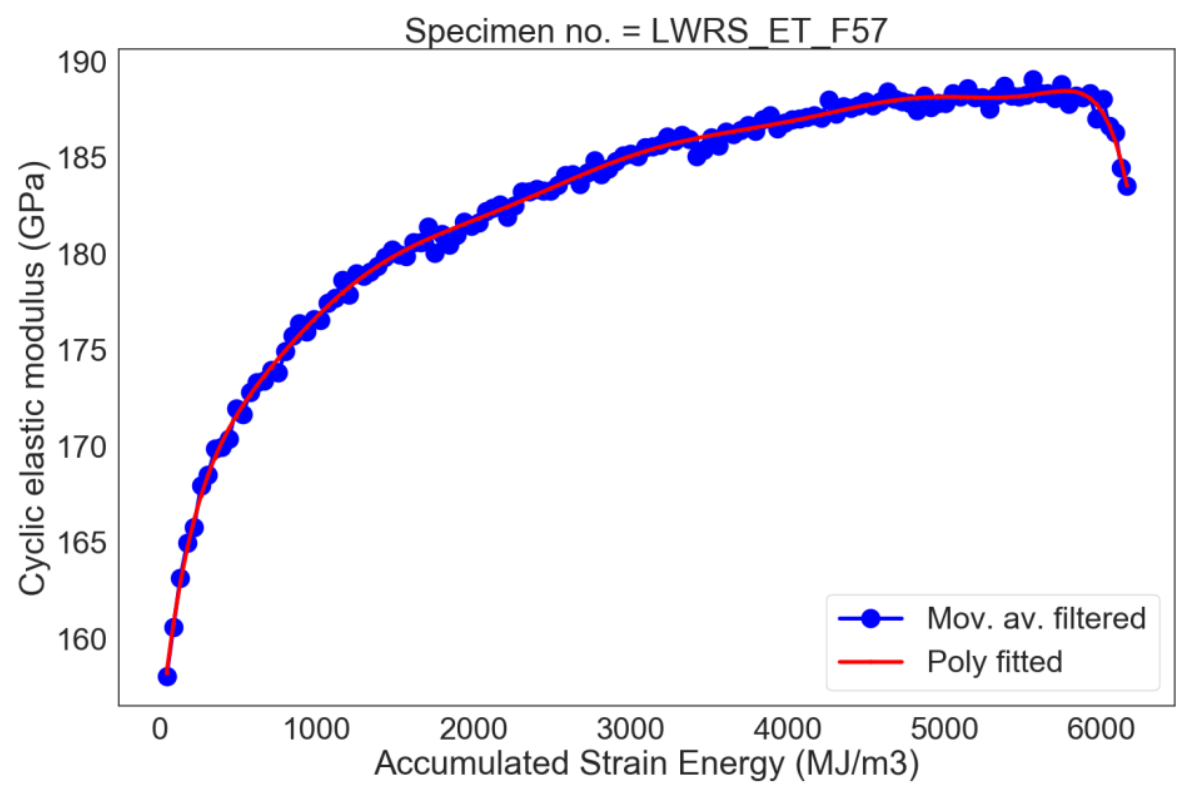

Figure 7. 37 Accumulated strain-energy versus elastic modulus, estimated based on the ET-F57 test data. 


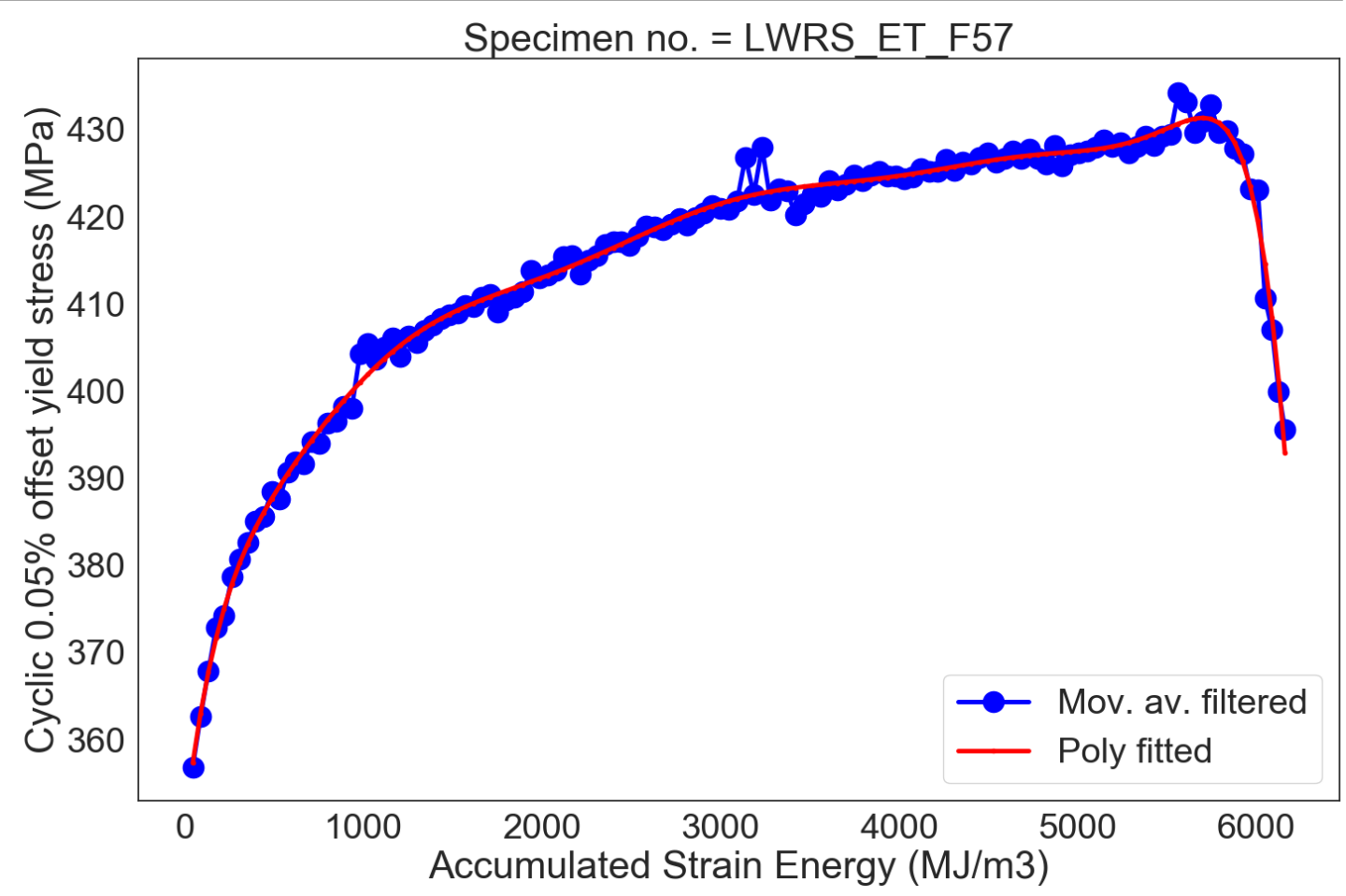

Figure 7. 38 Accumulated strain-energy versus $0.05 \%$ offset yield stress, estimated based on the ET-F57 test data.

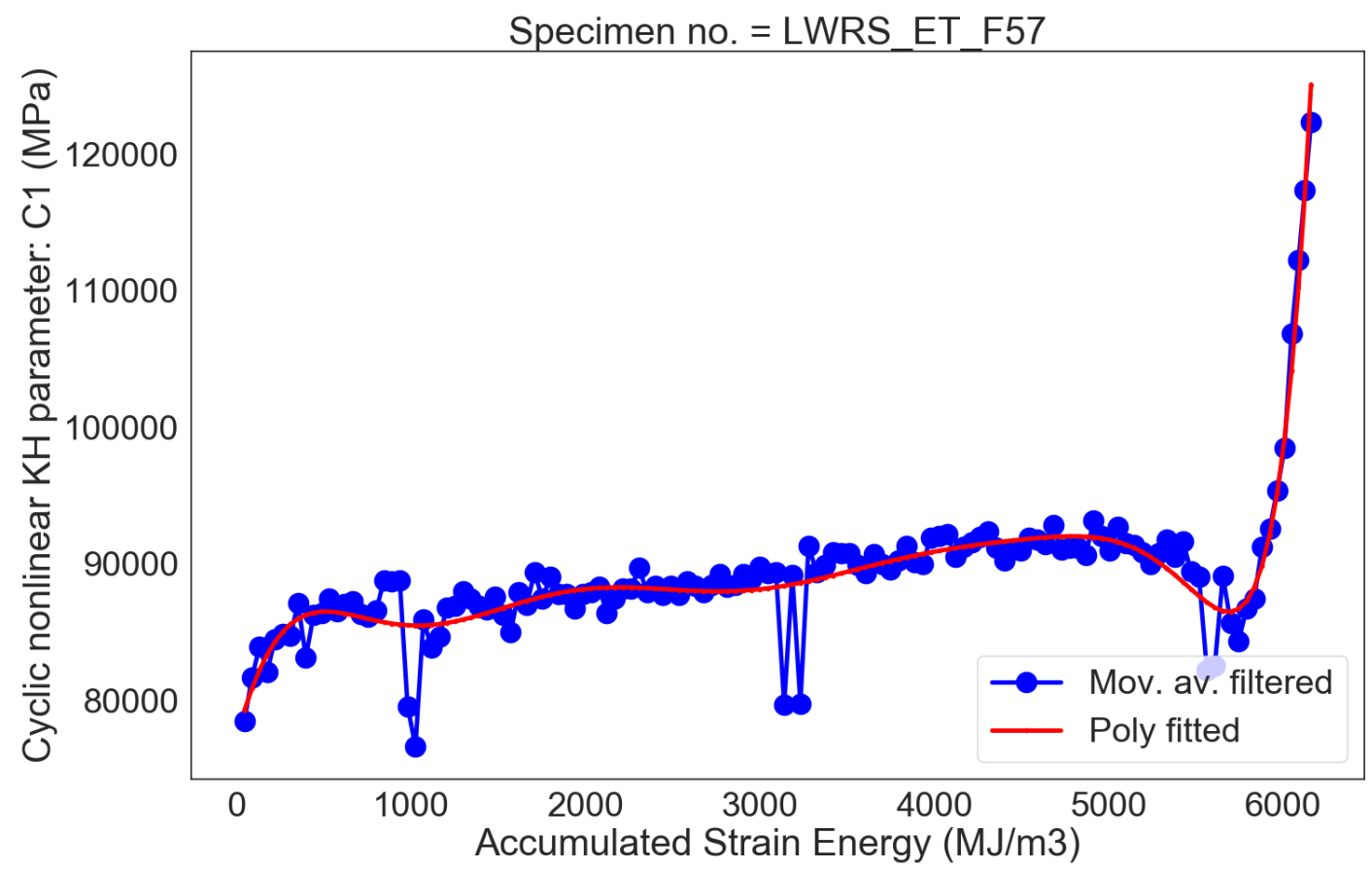

Figure 7. 39 Accumulated strain-energy versus nonlinear kinematic hardening parameter C1, estimated based on the ET-F57 test data. 


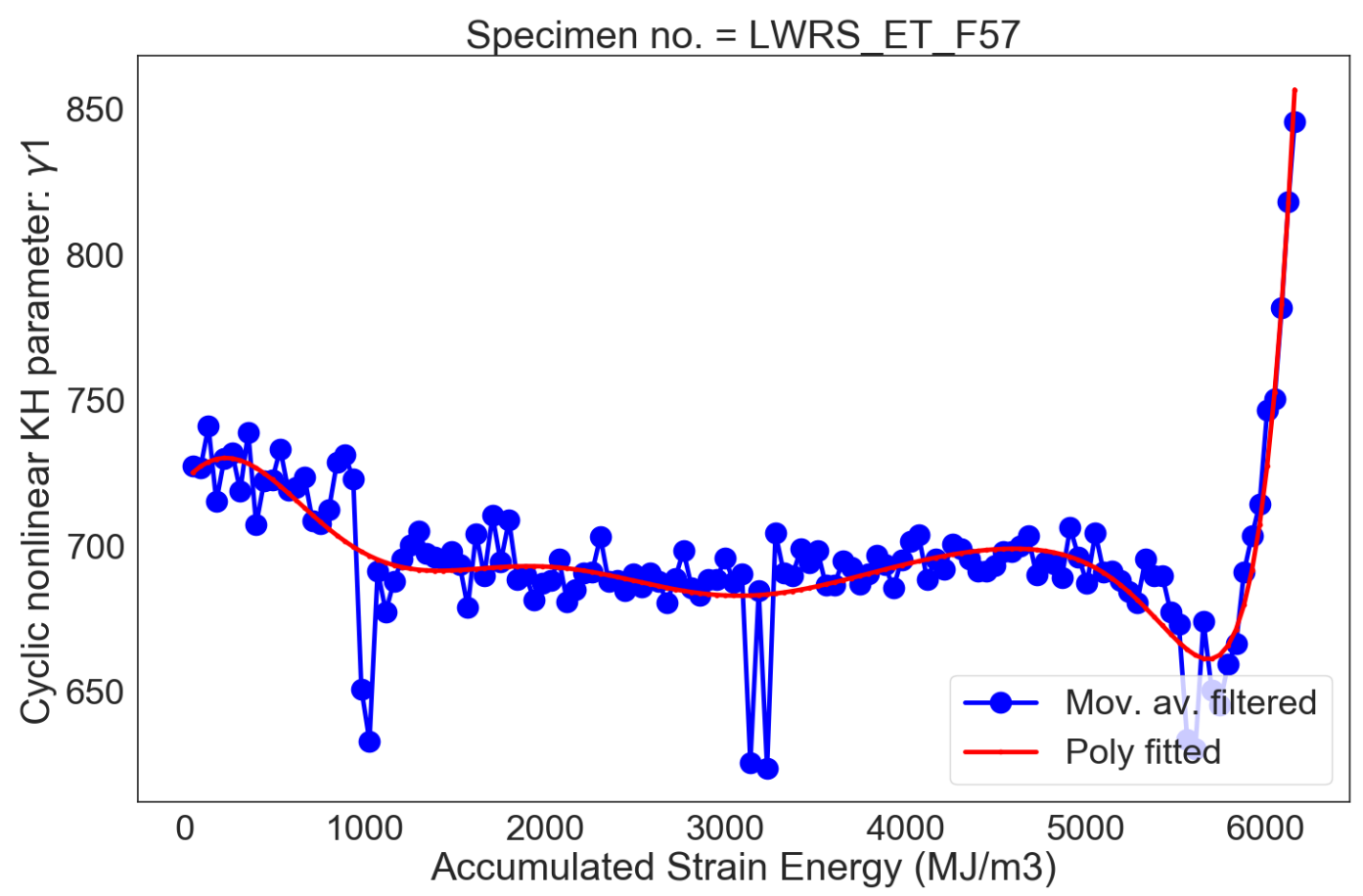

Figure 7. 40 Accumulated strain-energy versus nonlinear kinematic hardening parameter $\gamma 1$, estimated based on the ET-F57 test data.

7.5.2 Cyclic stress analysis results for ET-F57 specimen using accumulated strain-energy dependent material model properties

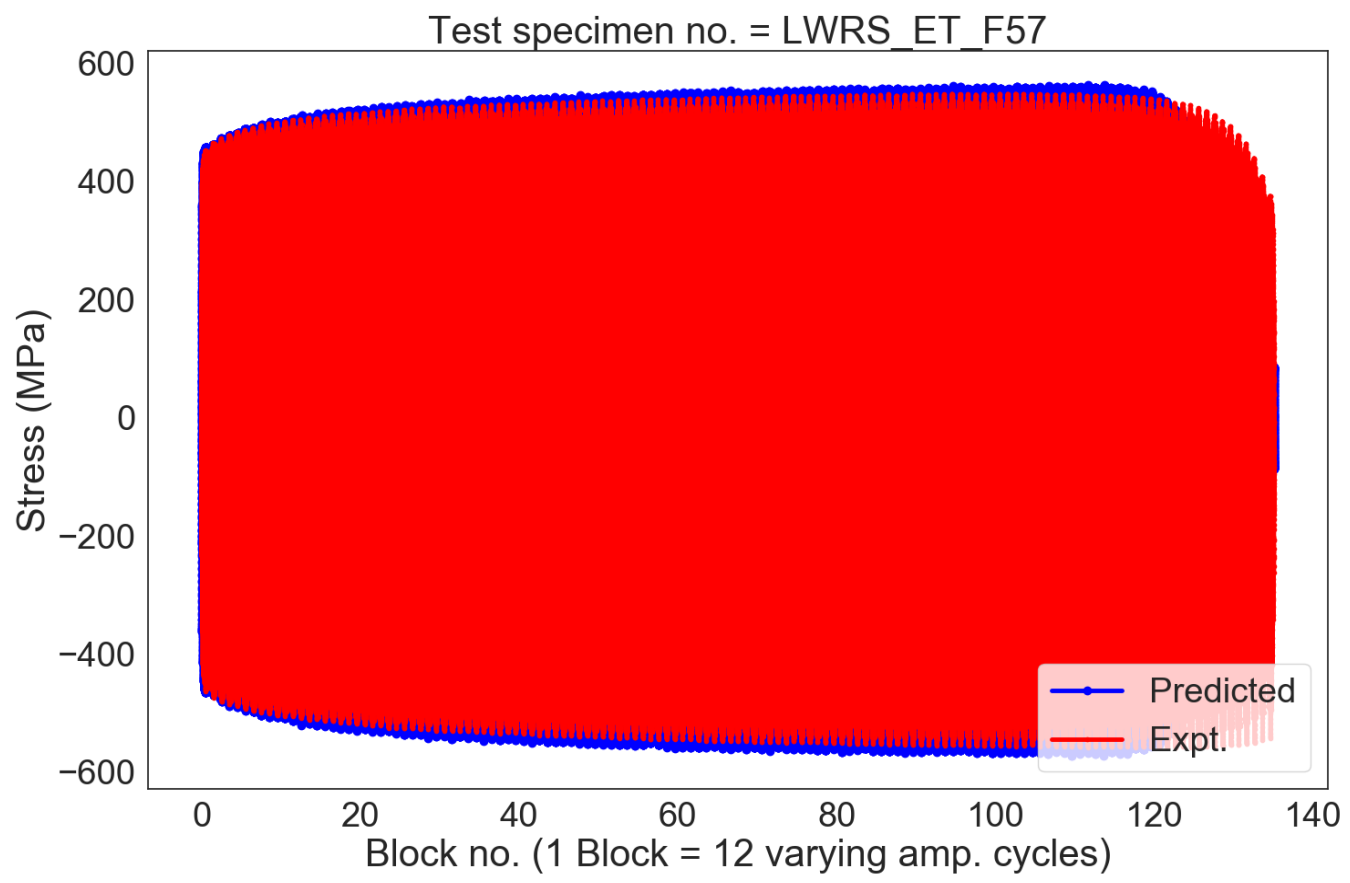

Figure 7. 41 Cycle-dependent properties based predicted versus experimentally observed cyclic stress for the entire fatigue life of ET-F57 test case. 


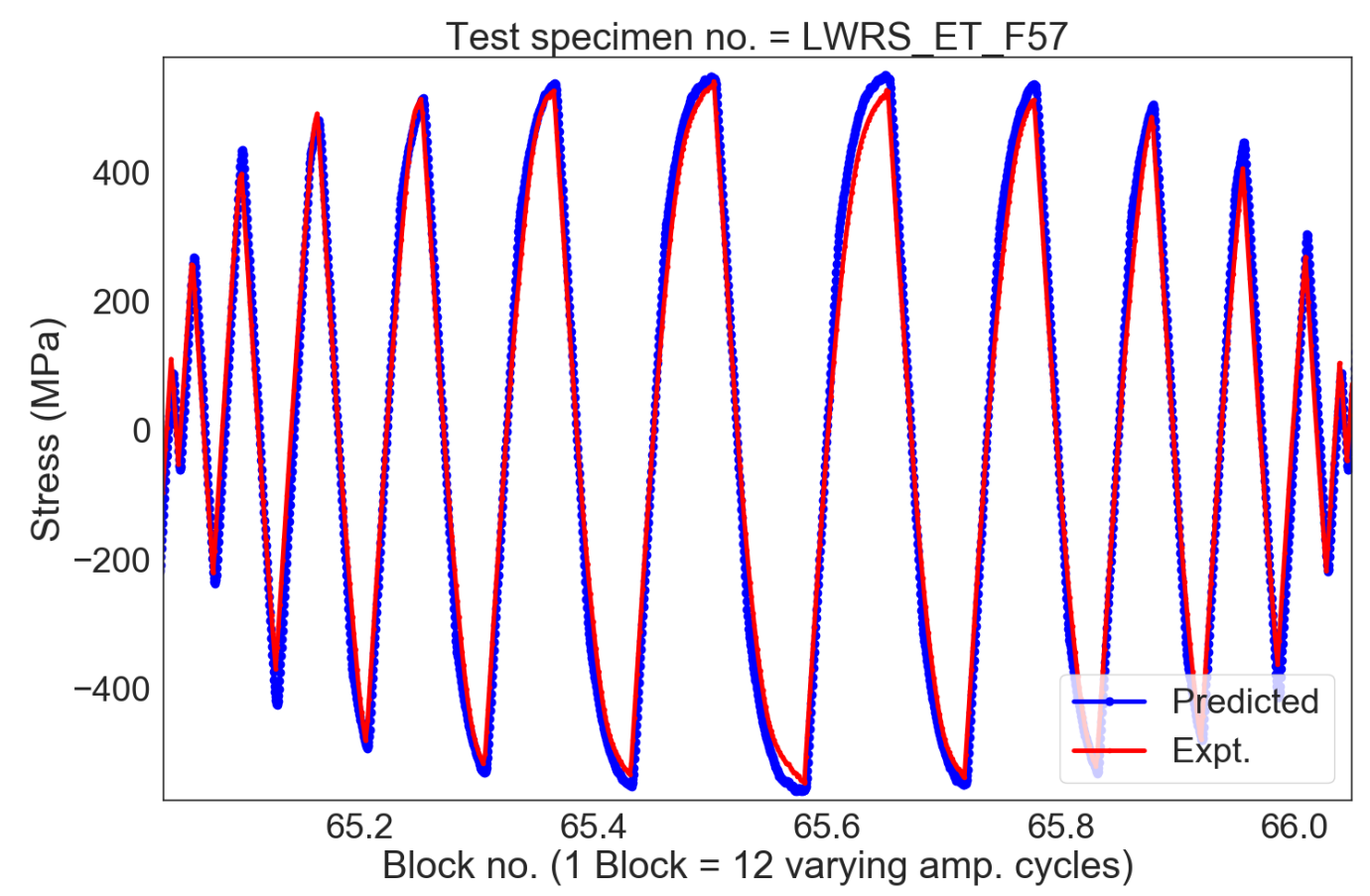

Figure 7. 42 Magnified version of Figure 7.41, approximately during the half-life of ET-F57 specimen.

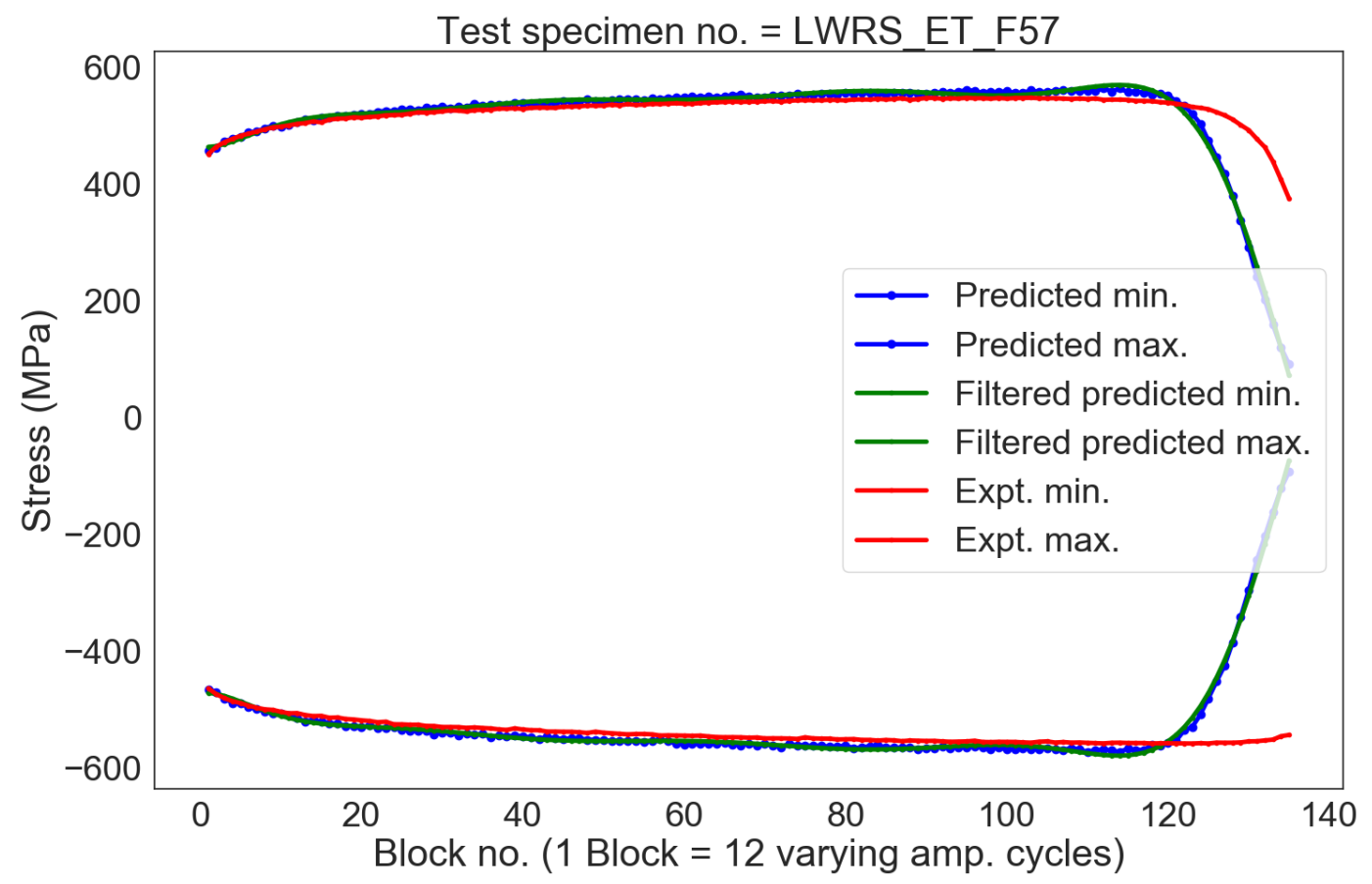

Figure 7. 43 Cycle-dependent properties based predicted versus experimentally observed max./min stress-amplitudes for the entire fatigue life of ET-F57 test case. 


\section{Demonstration of the Use of Cyclic-Plasticity Material Parameters in 3D FE Model of a PWR Surge-line Nozzle}

In this section, we demonstrate the use of cyclic plasticity material parameters using a commercial software such as ABAQUS. Using the estimated material parameters and a 3D finite element model, stress analysis was performed for a surge-line nozzle. This was to check how the cyclic plasticity material parameters affect the stress analysis results.

\subsection{FE Model of PWR Surge-line Nozzle}

We used our earlier developed 3D FE model of the PWR surge-line nozzle and pressurizer-bottomhead assembly to perform the present discussed stress analysis. For details of the model it is suggested to refer to our earlier publication [17]. Figure 8.1 shows the partial CAD model of PWR pressure control system showing the location of the surge line nozzle. However, we have only considered the portion of the surge-line, its nozzle and portion of the pressurizer (that is only considering the bottom head of the pressurizer). Figure 8.2 shows the CAD model of the assembly used in the discussed stress analysis model. Figure 8.3 shows the cut section of the nozzle assembly showing different material systems. Figure 8.4 shows the finite element mesh. As can be seen from Figure 8.3, the assembly consists of 5 material systems: such as 316SS base metal, 508LAS base metal, 316SS-316SS similar metal weld (SMW), 316SS-508LAS dissimilar-metal-weld (DMW) both filler and butter weld regions. This requires different material models to accurately perform the stress analysis. In our earlier work [17] we developed the tensile test-based material models for all the above mentioned 5 material system. The tensile test-based parameters can be used for firsthand stress analysis of a reactor component e.g. of the mentioned nozzle. However, as demonstrated in section 7, tensile test based fixed properties do not capture the material hardening/softening under cyclic loading. For more accurate stress analysis, we need to use cyclic plasticity-based material models. In the present discussed results, we used the cyclicplasticity material parameters for DMW filler weld only. Whereas, for other material, tensile test-based properties were used. This is for the present demonstration purpose only, to isolate out the effect of cyclic-plasticity material model use. The details of the tensile properties can be found from [17], whereas the cyclic plasticity based DMW filler weld properties (which are used in this work) are already presented in section 6 . Two cases of simulations were performed considering $1^{\text {st }}$ and $68^{\text {th }}$ block (halflife) properties of DMW filler weld (ET-F57) data. The temperature for the entire nozzle assembly assumed an isothermal temperature of $300{ }^{\circ} \mathrm{C}$. This is to be consistent with test temperature of ET-F57 specimen. However, note that for stress analysis of nozzle assembly under actual temperature varying thermal-mechanical loading transients, temperature dependent material models must be used. The ANL team develop both the tensile and cyclic material models keeping in mind its direct use by industry. The estimated material properties automatically stored into a SQL based database file which can be shared with US nuclear industry and regulatory agency on request. The material models can be directly used by commercially available software such as ABAQUS with one example shown in Figure 8.5. 


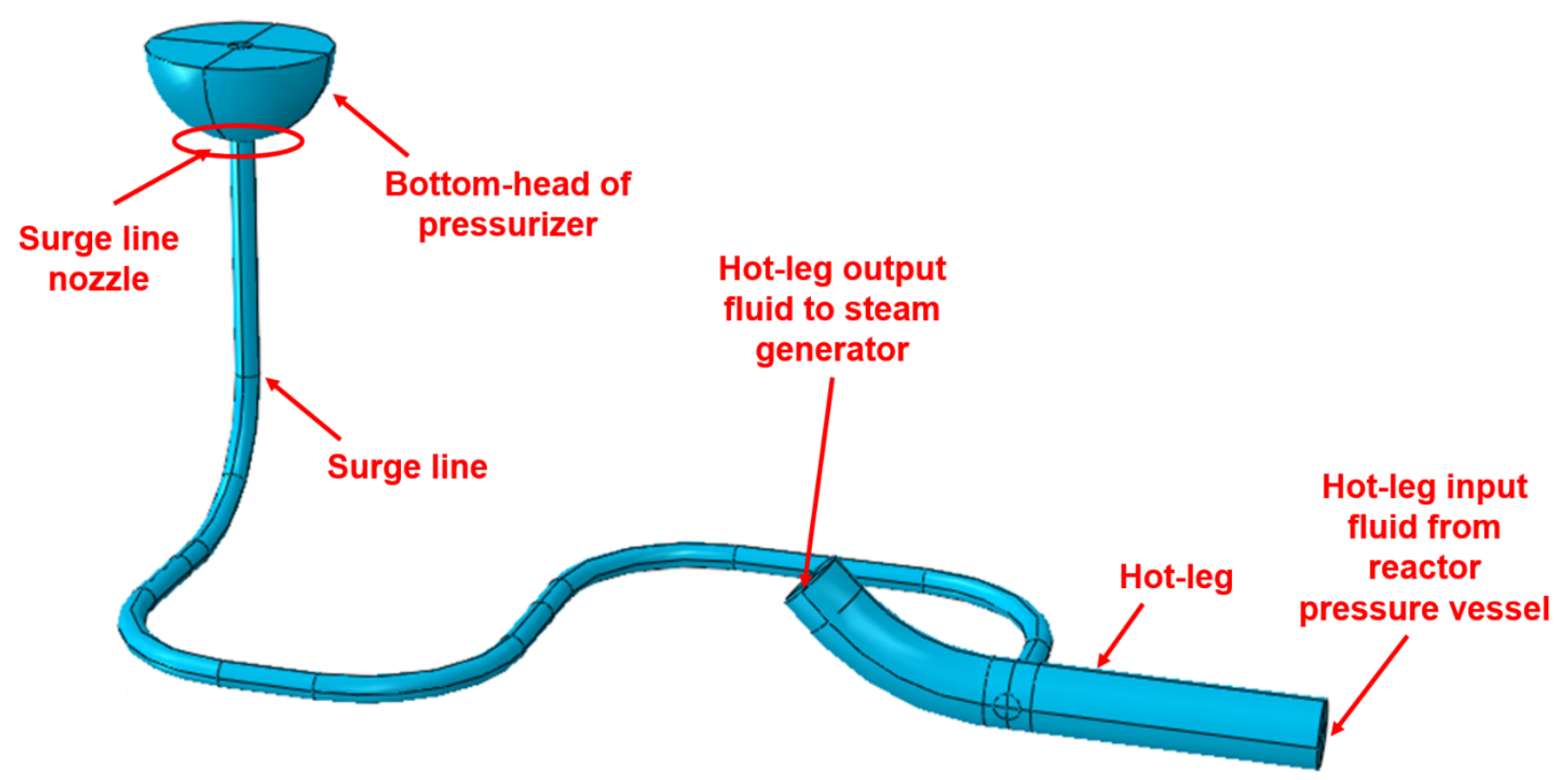

Figure 8. 1 Partial CAD model of PWR pressure control system showing the location of the surge line nozzle.

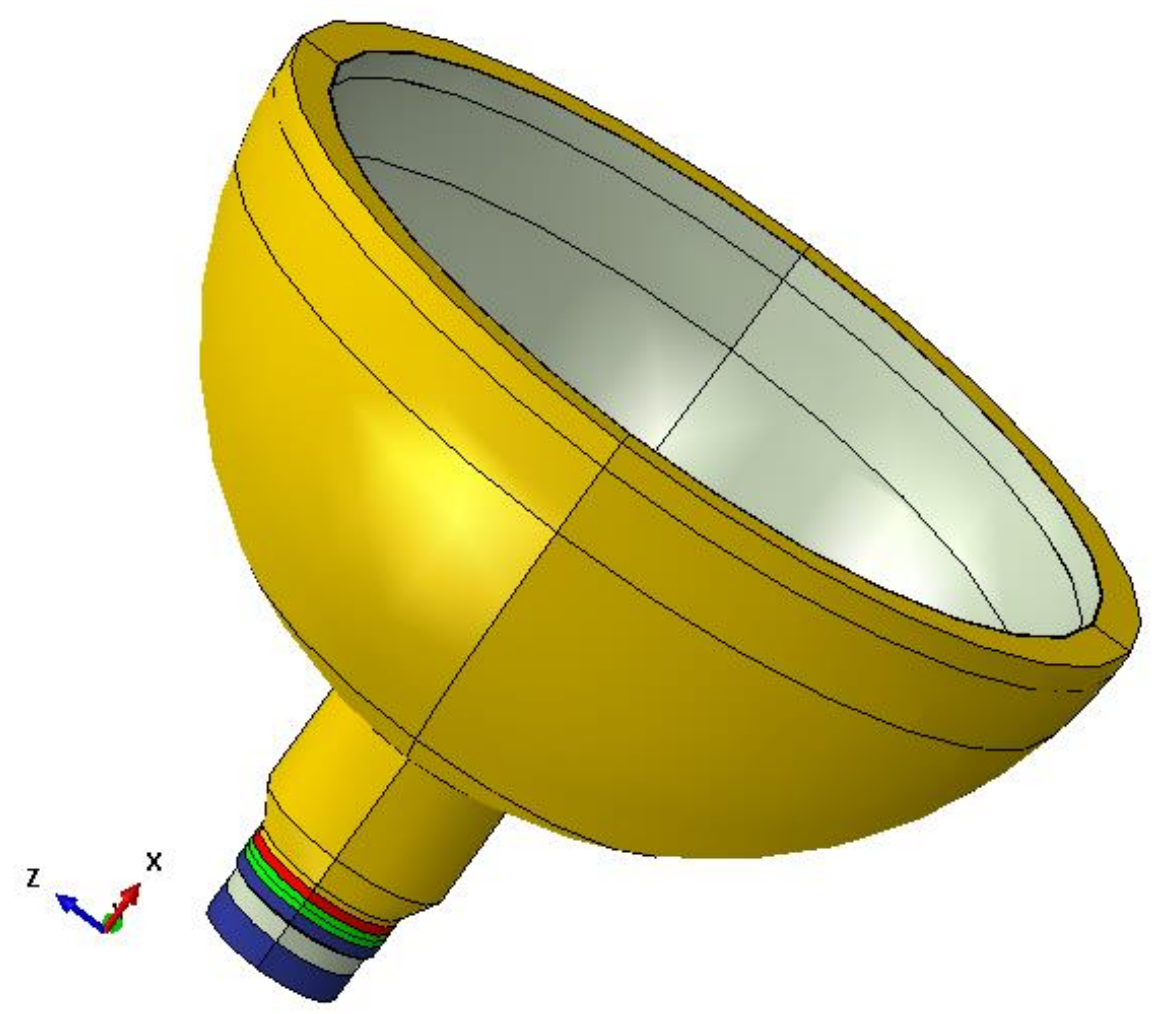

Figure 8. 2 CAD model of surge line nozzle and bottom head of pressurizer. 


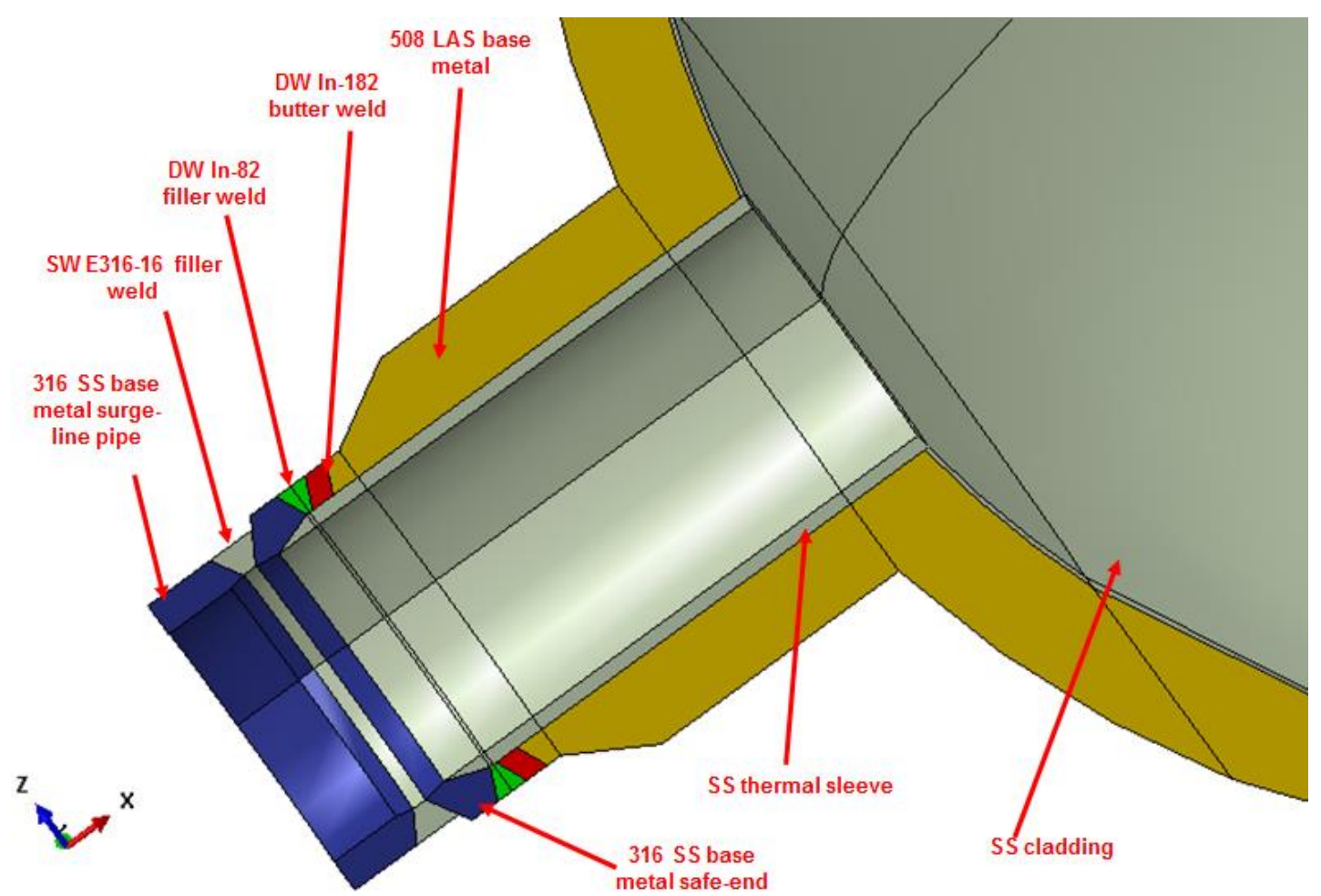

Figure 8. 3 Cut section of the nozzle assembly showing different material systems.

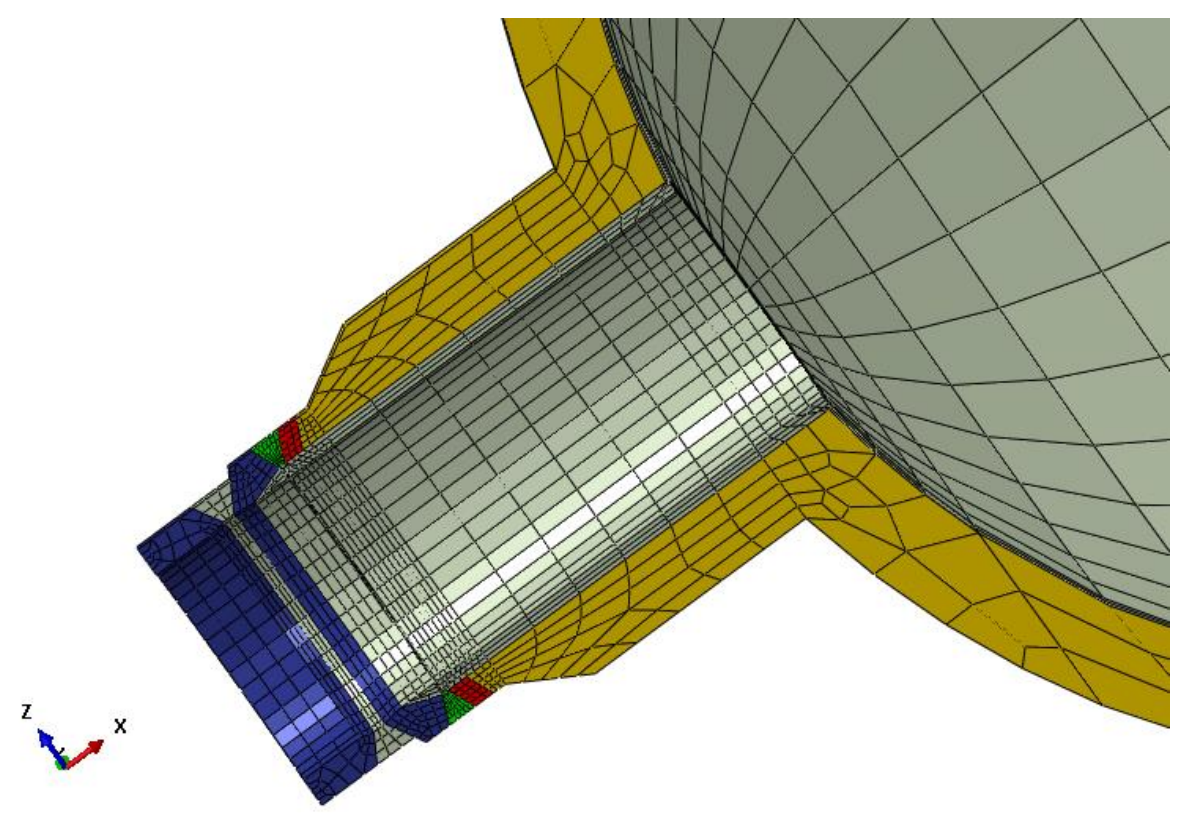

Figure 8. 4 Cut section of the nozzle assembly finite element mesh. 


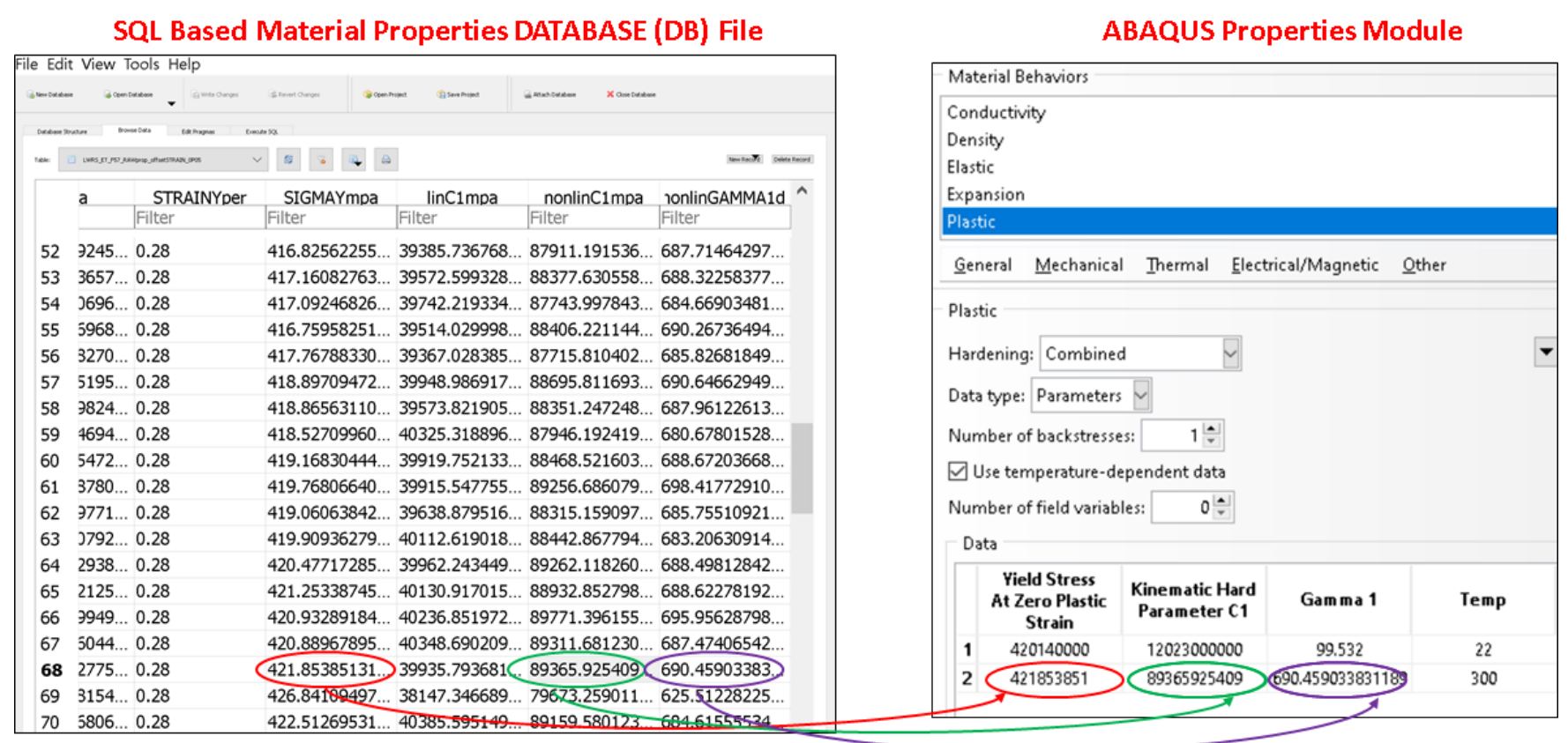

Figure 8. 5 Demonstration showing how the estimated material properties can directly be used in a commercially available software such as ABAQUS.

\subsection{FE stress analysis results with respect to ET-F57 block-1 and block-68 (half-life) properties}

In the discussed demonstration FE models, we used a displacement loading to generate similar strain profile as the ET-F57 specimen. The displacement applied at central plane of DMW filler weld region. The location and direction of the applied displacement loading can be seen from Figure 8.6. Whereas Figure 8.7 shows the applied 1-block of displacement. Figure 8.8 shows the comparison of FE simulated cycle versus strain at a typical element of DMW filler weld (of the PWR surge nozzle) subjected to ETF57 $1^{\text {st }}$ block and $68^{\text {th }}$ block (half-life) material properties. Figures 8.9 and 8.10 show the corresponding cycle versus stress and strain versus stress (hysteresis) plots, respectively. Figure 8.11 shows the example Von. Mises stress contour of PWR surge-line nozzle at a typical instance of the applied variable amplitude displacement, while subjected to ET-F57 half-life ( $68^{\text {th }}$ block) material properties. From Figures 8.8 to 8.10, it can be seen that substantial difference exists in predicted stress-strain results between $1^{\text {st }}$ block and $68^{\text {th }}$ block properties-based models, although both models were simulated under same displacement loading (Figure 8.7). This is because of multi-axial stress/strain-redistribution which is a function input material property. Nevertheless, this demonstration results show that component stress analysis results can substantially vary if cyclic-plasticity based property is used. Since it is demonstrated that the all the material properties (e.g. elastic modulus, yield stress, kinematic hardening parameters $\mathrm{C} 1$ and $\gamma 1$ ) substantially vary over fatigue cycles, it may be worth considering those cyclic varied properties for more accurate life estimation of reactor components particularly for long-term licensing of nuclear reactors. 


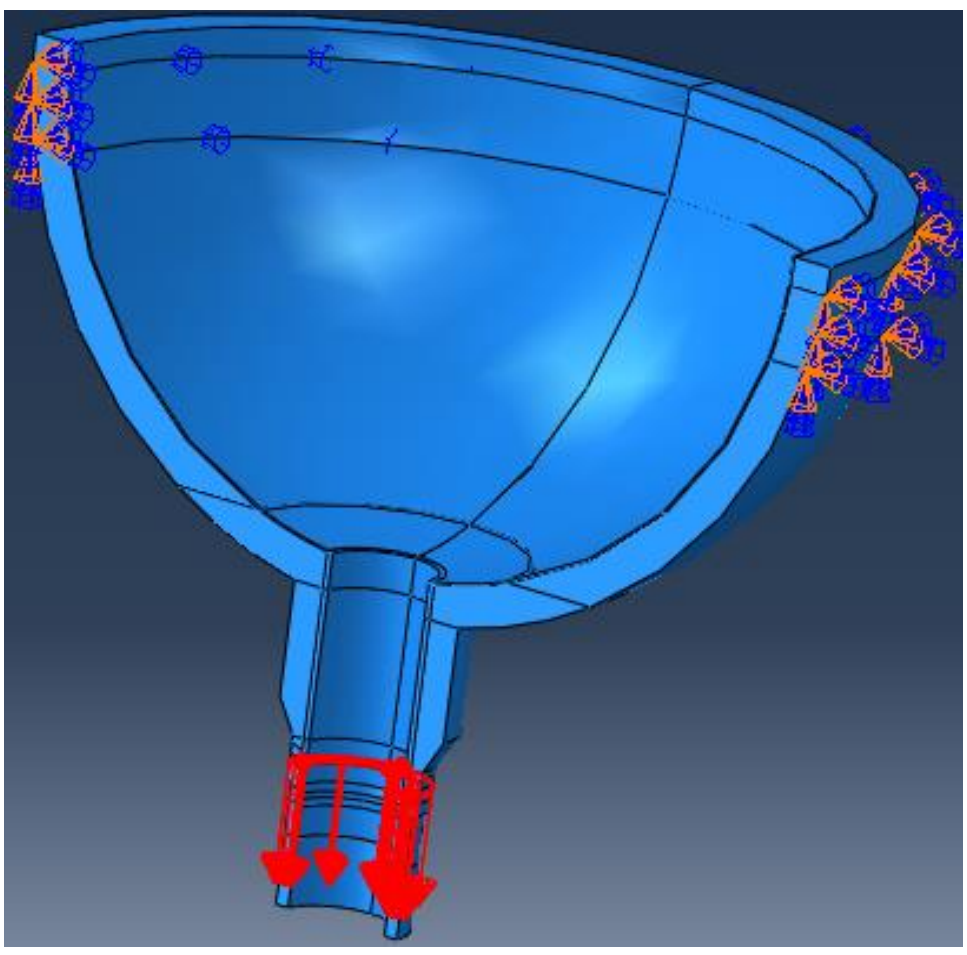

Figure 8. 6 Cut section of nozzle assembly showing the location and direction of the applied variableamplitude displacements to simulate variable-amplitude strain at the DMW filler weld.

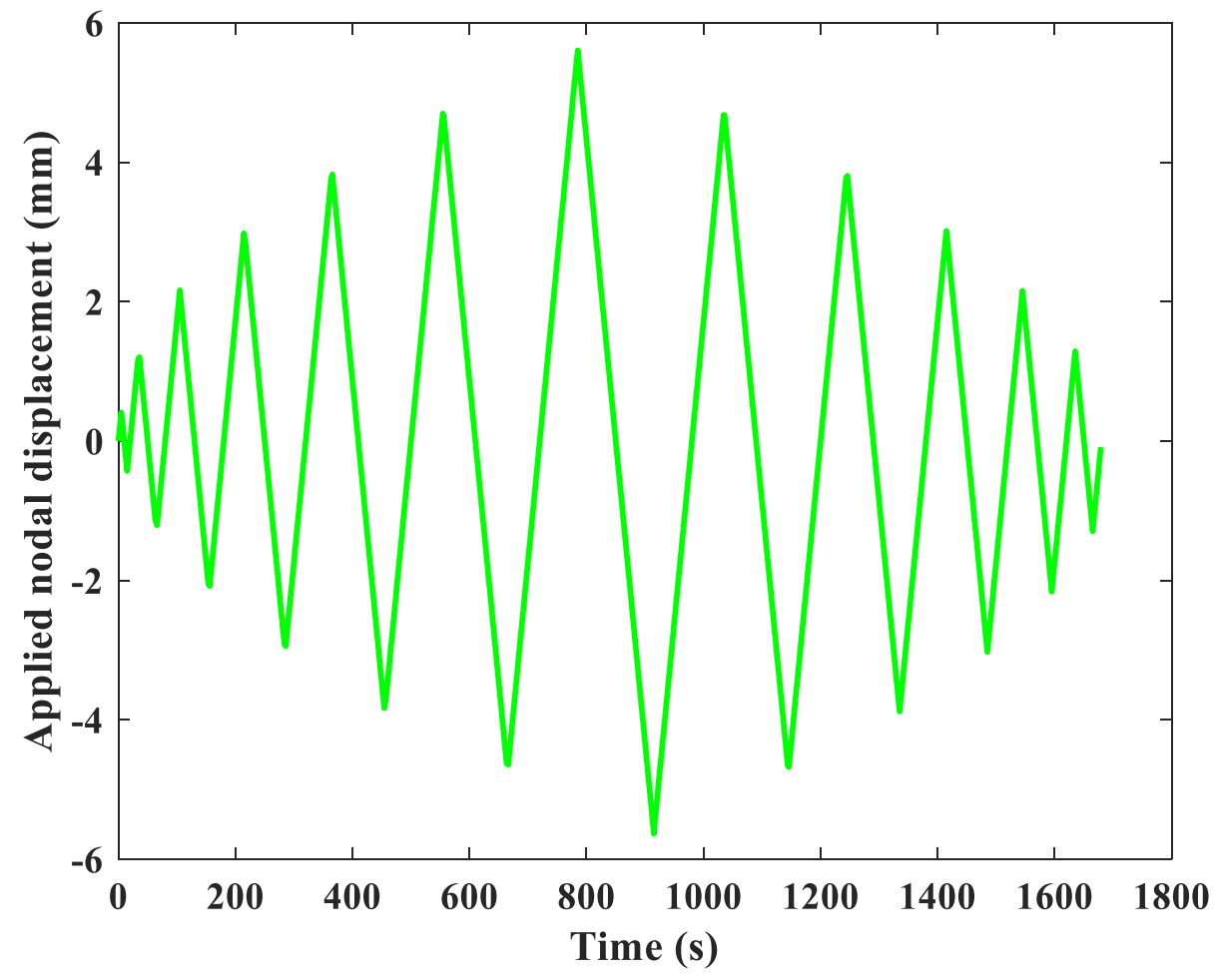

Figure 8. 7 Applied 1-block of displacement at the circular mid-plane of DMW filler region. 


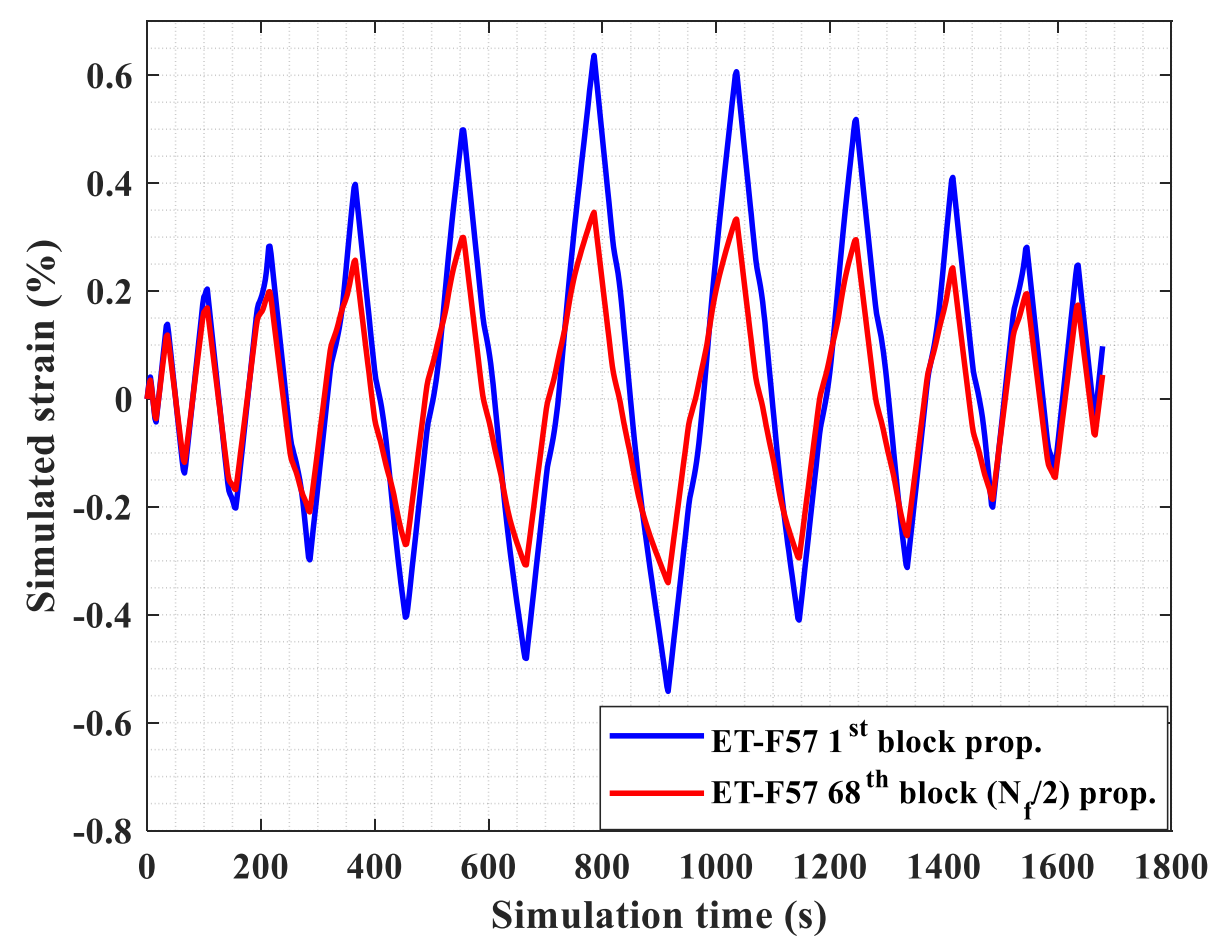

Figure 8. $8 \mathrm{FE}$ simulated cycle versus strain at a typical element of DMW filler weld (of the PWR surge nozzle) subjected to ET-F57 $1^{\text {st }}$ block and $68^{\text {th }}$ block (half-life) material properties.

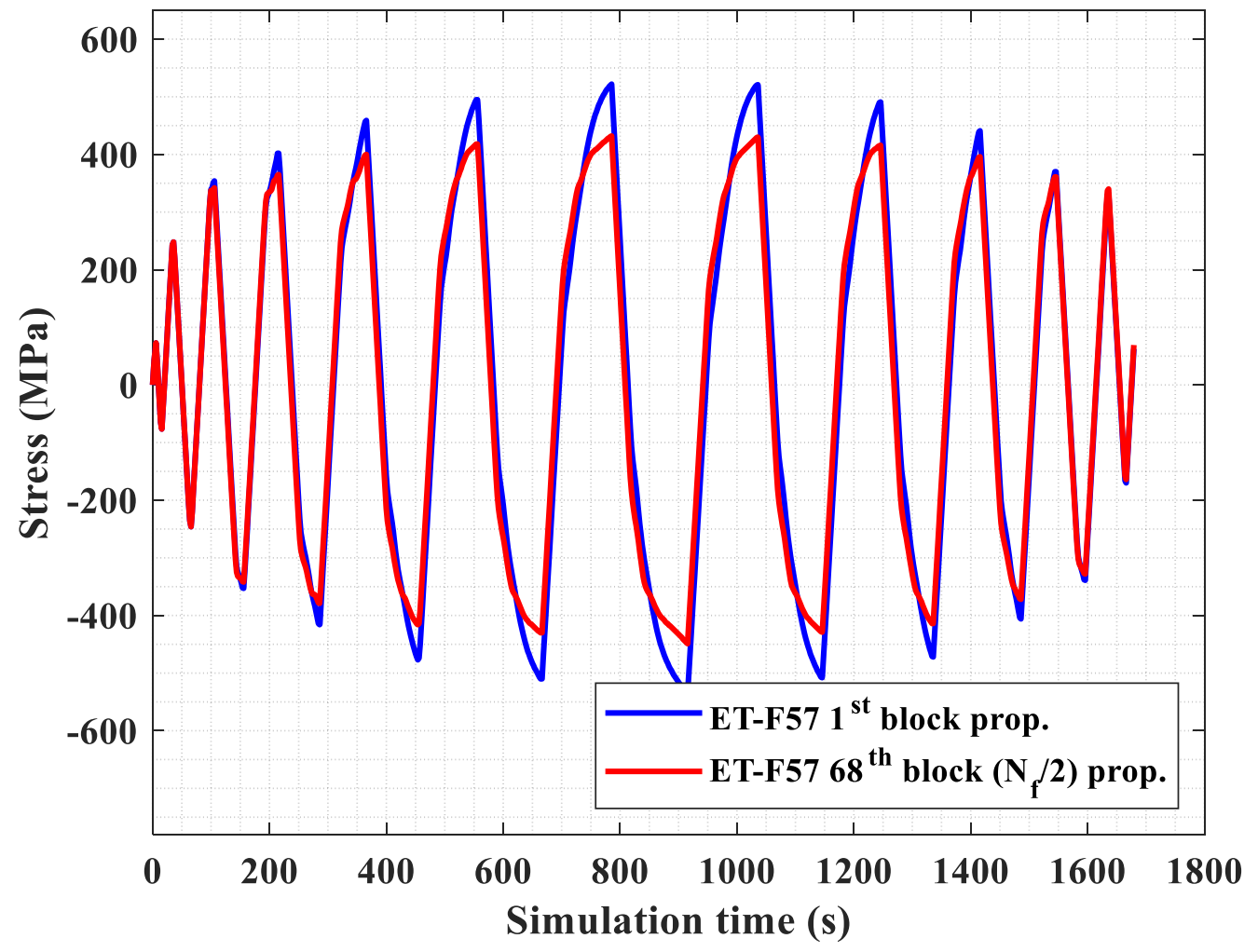

Figure 8. 9 FE simulated cycle versus stress at a typical element of DMW filler weld (of the PWR surge nozzle) subjected to ET-F57 $1^{\text {st }}$ block and $68^{\text {th }}$ block (half-life) material properties. 


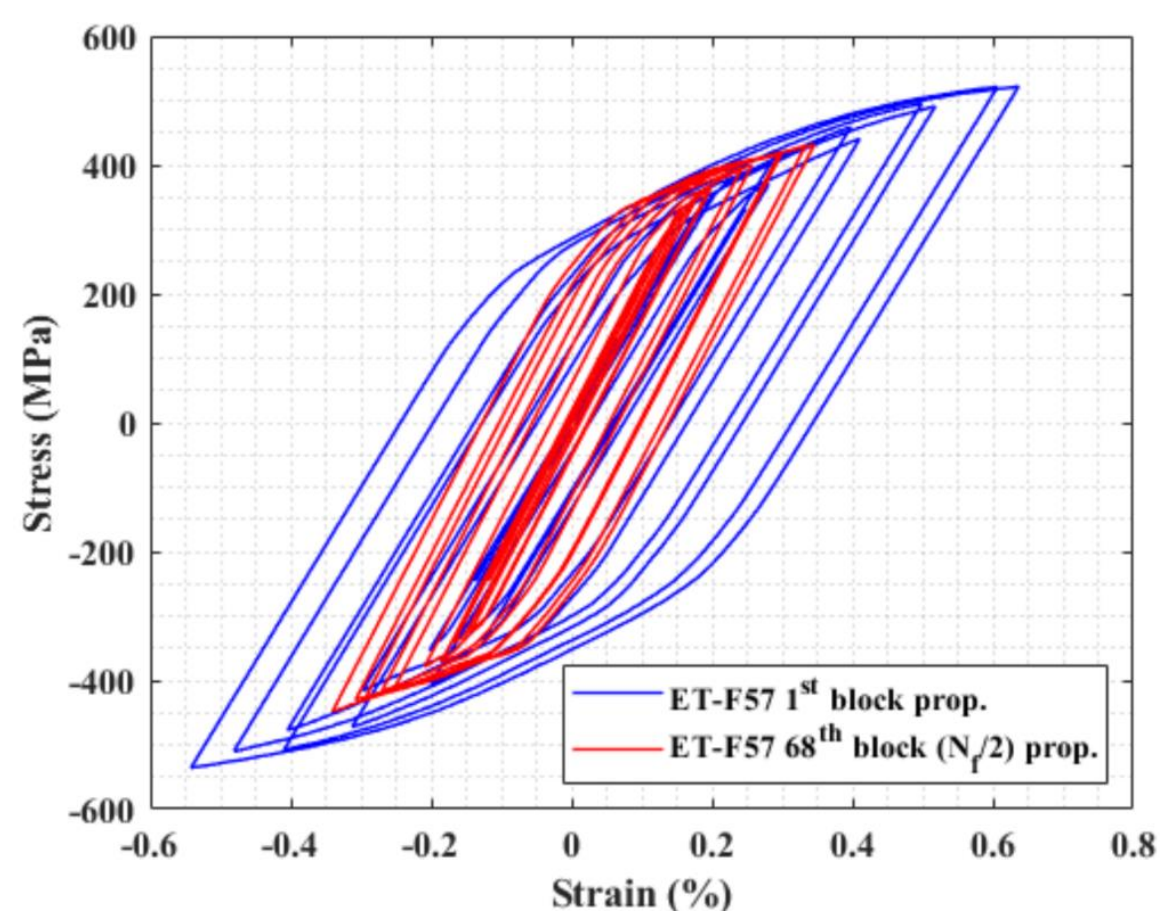

Figure 8. 10 FE simulated stress versus strain at a typical element of DMW filler weld (of the PWR surge nozzle) subjected to ET-F57 $1^{\text {st }}$ block and $68^{\text {th }}$ block (half-life) material properties.

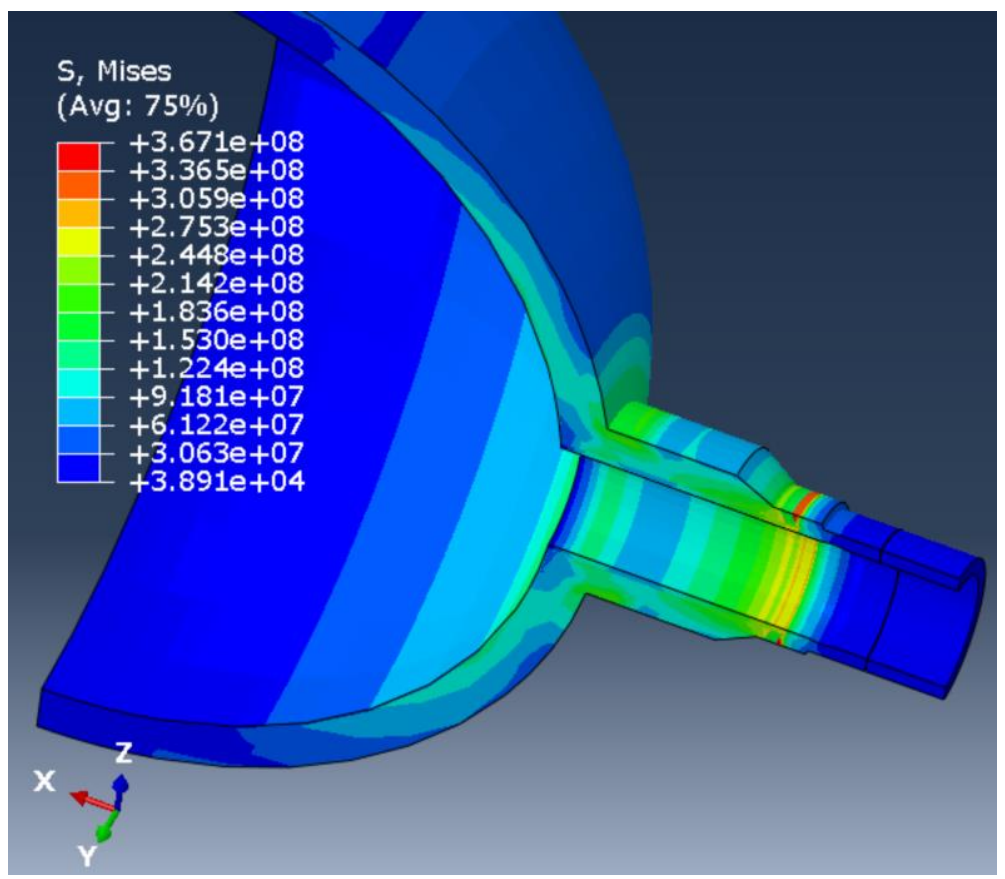

Figure 8. 11 Von. Mises stress (Pascal) contour of PWR surge-line nozzle at a typical instance of the applied variable amplitude displacement, while subjected to ET-F57 half-life (68 ${ }^{\text {th }}$ block) material properties. 


\section{Markov-chain-Monte-Carlo Based Probabilistic-Time-Series State and Fatigue Life Estimation of DMW}

In this section, we present results related to probabilistic life estimation of DMW. Often there exists a large scatter in fatigue life data even the underlying tests are conducted using specimen fabricated from same material and tested under same loading and environment conditions. The scatter in fatigue life is due to scatter in material microstructure, test specimen surface finish and their interactions with loading and environmental conditions. The issue of statistical scatter is more severe for welded materials due to possible nonhomogeneous laying of weld layers. Hence it is essential to predict the life of a welded component both deterministically and probabilistically rather than following only a deterministic approach. In this section we present some probabilistic modeling results of DMW (both under in-air and PWR water environment). This is by using Markov chain Monte Carlo (MCMC) method, details of which can be found from our earlier work [6]. In the present discussed work, we used the MCMC model to estimate probabilistic time-series of cycle versus maximum stress amplitudes. From the estimated time-series scatter-band, the probabilistic life of the DMW are estimated for a given failure criteria such as using the ASME 25\% load drop criteria. This type of simulation-based approach may help to reduce the over dependence on test-based approach. For example, statistical estimation of metal under a single environment and loading spectra might requires tens to hundreds of fatigue tests. Conducting these many fatigue tests under different loading and environmental conditions not only is immensely expensive but also time consuming. Nevertheless, the purpose of this section is to demonstrate the use of MCMC technique for estimating the probabilistic life of DMW under a given load and environmental conditions. We used the MCMC approach either using the model simulated data or experimentally observed data. As computational techniques for structural analysis improves (for example by capturing the cyclic evolution of material states as we demonstrated through analytical model described in section 7), the MCMC model can directly be based on simulated stress-strain states. However, we demonstrated MCMC model results both based on analytical model results and experimental model results. Following are the three cases which results are presented below:

1. MCMC model simulation based on ET-F57 analytical model results (i.e. using block versus simulated maximum stress data)

2. MCMC model simulation based on ET-F57 experimentally observed results (i.e. using block versus experimentally observed maximum stress data)

3. MCMC model simulation based on EN-F62 experimentally observed results (i.e. using block versus experimentally observed maximum stress data)

The related example results can be found below from Figures 9.1 to 9.6. Whereas, the Table 9.1 shows the summary of the model and experiment data based MCMC simulated probabilistic lives of DMW of the above-mentioned cases. From this table the MCMC simulation results based on model (cyclic plasticity based analytical model) data, are quite comparable with the MCMC simulation results based on experiment data. This shows the promise of a complete model/simulation-based approach to avoid over dependency on large-scale test-based programs, which are not only time consuming but also immensely expensive (particularly for conducting tens to hundreds of fatigue testing for different material under different loading and environmental conditions). From the Table 9.1 it can be found that 
the DMW specimen (subjected to EN-F62 test case loading amplitude and rate as given in section 3) can survive 358 cycles under PWR water environment, without any failure (or zero failure probability).

Table 9. 1 Summary of the model and experiment data based MCMC simulated probabilistic lives of DMW under different loading and environmental conditions.

\begin{tabular}{|c|c|c|c|c|c|}
\hline $\begin{array}{c}\text { MCMC } \\
\text { simulation } \\
\text { cases }\end{array}$ & $\begin{array}{c}\text { Load and } \\
\text { environment }\end{array}$ & $\begin{array}{c}0 \% \text { failure } \\
\text { probability } \\
\text { life of } \\
\text { DMW, } \\
\text { with } \\
\text { considering } \\
\text { ASME } \\
25 \% \text { load } \\
\text { drop } \\
\text { criteria }\end{array}$ & $\begin{array}{c}\text { Approximate } \\
50 \% \text { failure } \\
\text { probability } \\
\text { life of DMW, } \\
\text { with } \\
\text { considering } \\
\text { ASME } 25 \% \\
\text { load drop } \\
\text { criteria }\end{array}$ & $\begin{array}{c}100 \% \\
\text { failure } \\
\text { probability } \\
\text { life of } \\
\text { DMW, } \\
\text { with } \\
\text { considering } \\
\text { ASME } \\
25 \% \text { load } \\
\text { drop } \\
\text { criteria }\end{array}$ & $\begin{array}{c}\text { Experimentally } \\
\text { observed life } \\
\text { based on single } \\
\text { specimen test }\end{array}$ \\
\hline $\begin{array}{c}\text { ET-F57 } \\
\text { (Based on } \\
\text { analytically } \\
\text { predicted } \\
\text { cycle- } \\
\text { dependent } \\
\text { max. stress) }\end{array}$ & $\begin{array}{l}\text { Load: Gauge-area } \\
\text { strain-controlled } \\
\text { test (refer to } \\
\text { Figure } 3.10 \text { ), } \\
\text { Environment: } \\
300{ }^{\circ} \mathrm{C} \text {, in-air. }\end{array}$ & $\begin{array}{c}118 \text { blocks } \\
(1416 \\
\text { cycles })\end{array}$ & $\begin{array}{c}124 \text { blocks } \\
\text { (1488 cycles) }\end{array}$ & $\begin{array}{c}153 \text { blocks } \\
(1836 \\
\text { cycles })\end{array}$ & $\begin{array}{c}135 \text { blocks } \\
\text { (1620 cycles) }\end{array}$ \\
\hline $\begin{array}{c}\text { ET-F57 } \\
\text { (Based on } \\
\text { experimentally } \\
\text { observed } \\
\text { cycle- } \\
\text { dependent } \\
\text { max. stress) }\end{array}$ & $\begin{array}{c}\text { Load: Gauge-area } \\
\text { strain-controlled } \\
\text { test (refer to } \\
\text { Figure } 3.10 \text { ), } \\
\text { Environment: } \\
300{ }^{\circ} \mathrm{C} \text {, in-air. }\end{array}$ & $\begin{array}{c}98 \text { blocks } \\
(1176 \\
\text { cycles })\end{array}$ & $\begin{array}{c}116 \text { blocks } \\
\text { (1392 cycles) }\end{array}$ & $\begin{array}{c}162 \text { blocks } \\
(1944 \\
\text { cycles })\end{array}$ & $\begin{array}{c}135 \text { blocks } \\
\text { (1620 cycles) }\end{array}$ \\
\hline $\begin{array}{c}\text { EN-F62 } \\
\text { (Based on } \\
\text { experimentally } \\
\text { observed } \\
\text { cycle- } \\
\text { dependent } \\
\text { max. stress) }\end{array}$ & $\begin{array}{l}\text { Load: AI-guided- } \\
\text { pull-rod- } \\
\text { displacement } \\
\text { control (refer to } \\
\text { Figure } 3.14 \text { and } \\
\text { section } 4 \text { ), } \\
\text { Environment: } \\
300^{\circ} \mathrm{C}, \text { PWR } \\
\text { water }\end{array}$ & 358 cycles & 535 cycles & $\begin{array}{c}1044 \\
\text { cycles }\end{array}$ & 842 cycles \\
\hline
\end{tabular}




\subsection{Results: MCMC Model Simulation Based on Data of ET-F57 Analytical Model (with Accumulated} Strain Energy Dependent Cyclic-Plasticity Material Properties)

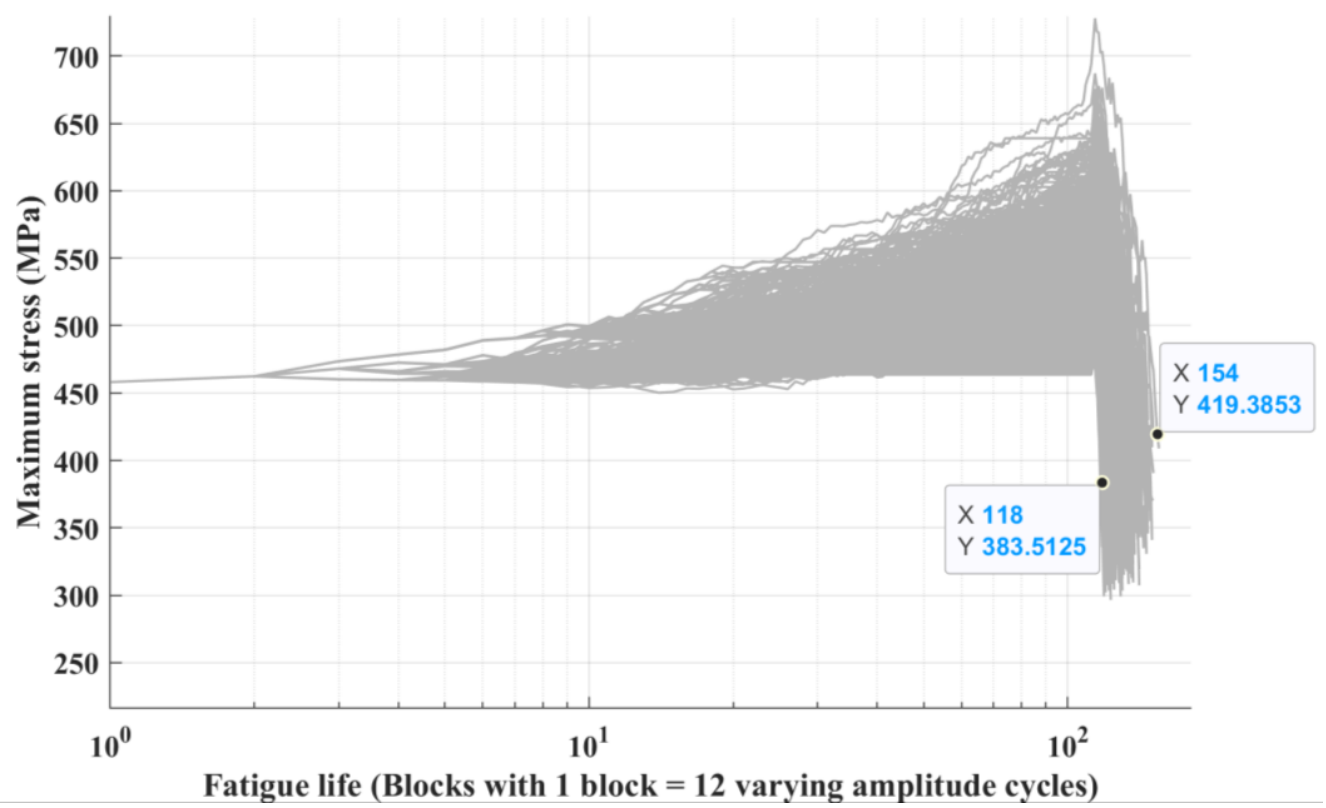

Figure 9. 1 MCMC estimated loading block versus the scatter-band of the analytically simulated maximum stress amplitude of ET-F57 test case.

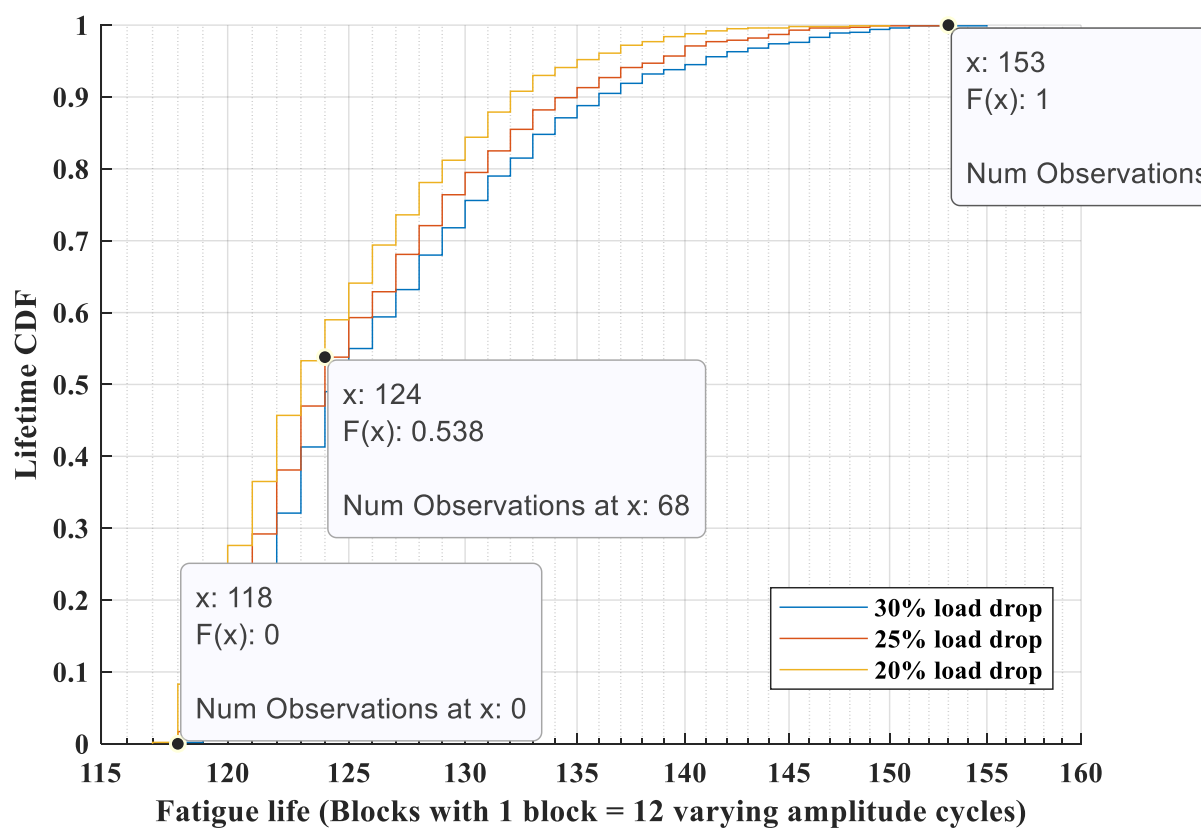

Figure 9. 2 Loading block versus estimated lifetime CDF, estimated based on the scatter-band shown in Figure 9.1 and using different load-drop failure criterion. 


\subsection{Results: MCMC Model Simulation Based on ET-F57 In-air Fatigue Test Data}

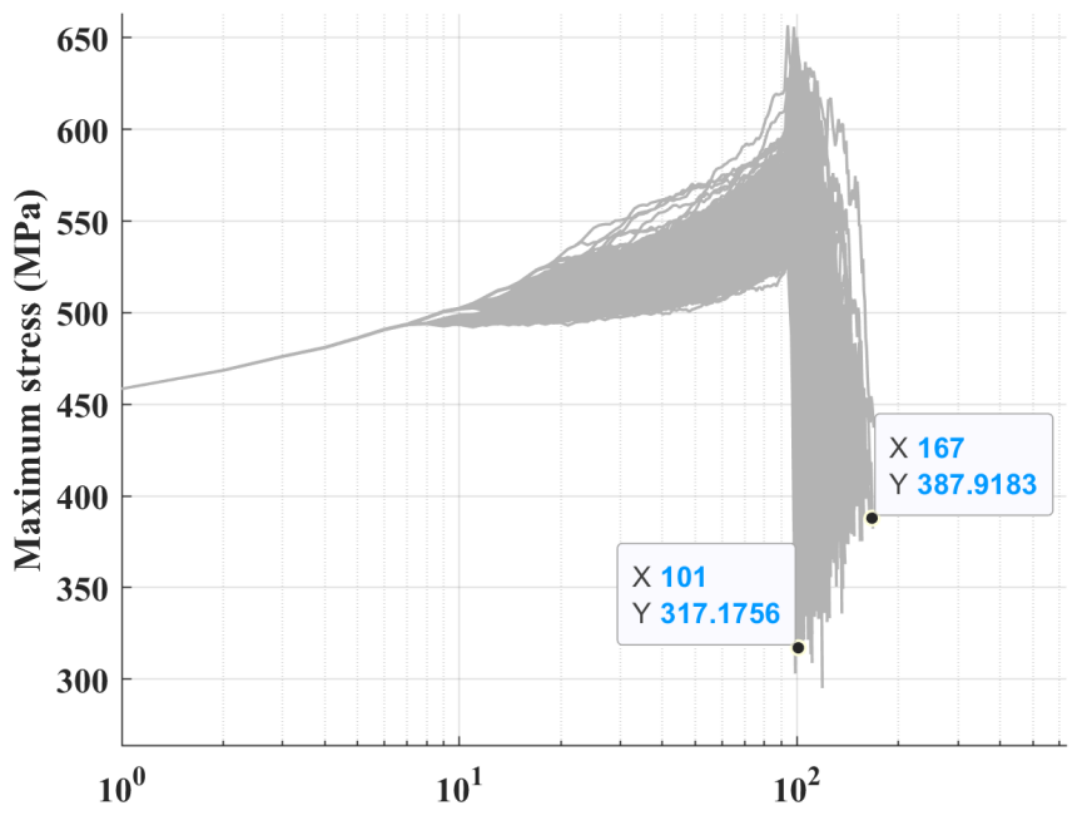

Fatigue life (Blocks with 1 block $=12$ varying amplitude cycles)

Figure 9. 3 MCMC estimated loading block versus the scatter-band of experimentally observed maximum stress amplitude of ET-F57 test case.

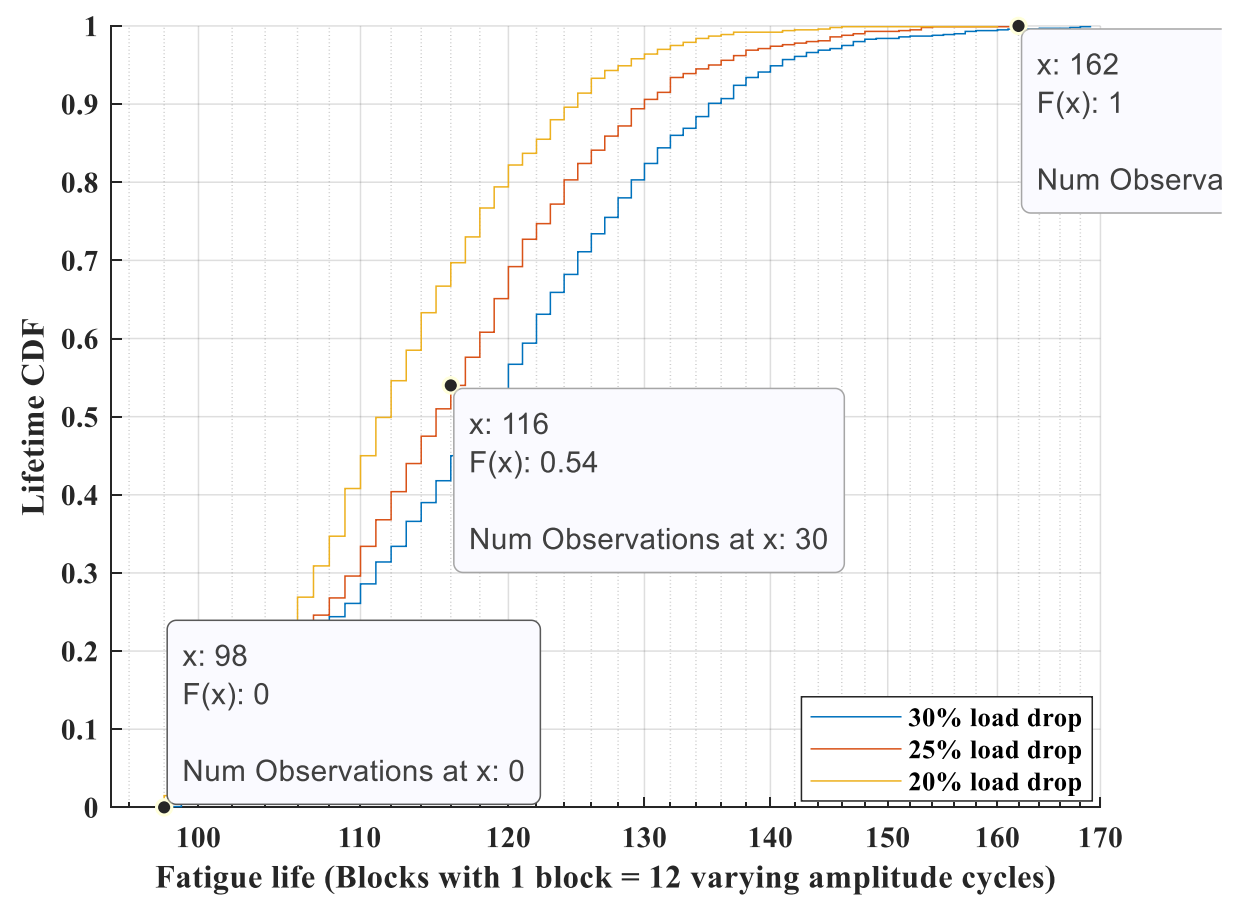

Figure 9. 4 Loading block versus estimated lifetime CDF, estimated based on the scatter-band shown in Figure 9.3 and using different load-drop failure criterion. 


\subsection{Results: MCMC Model Simulation Based on EN-F62 PWR-water Fatigue Test Data}

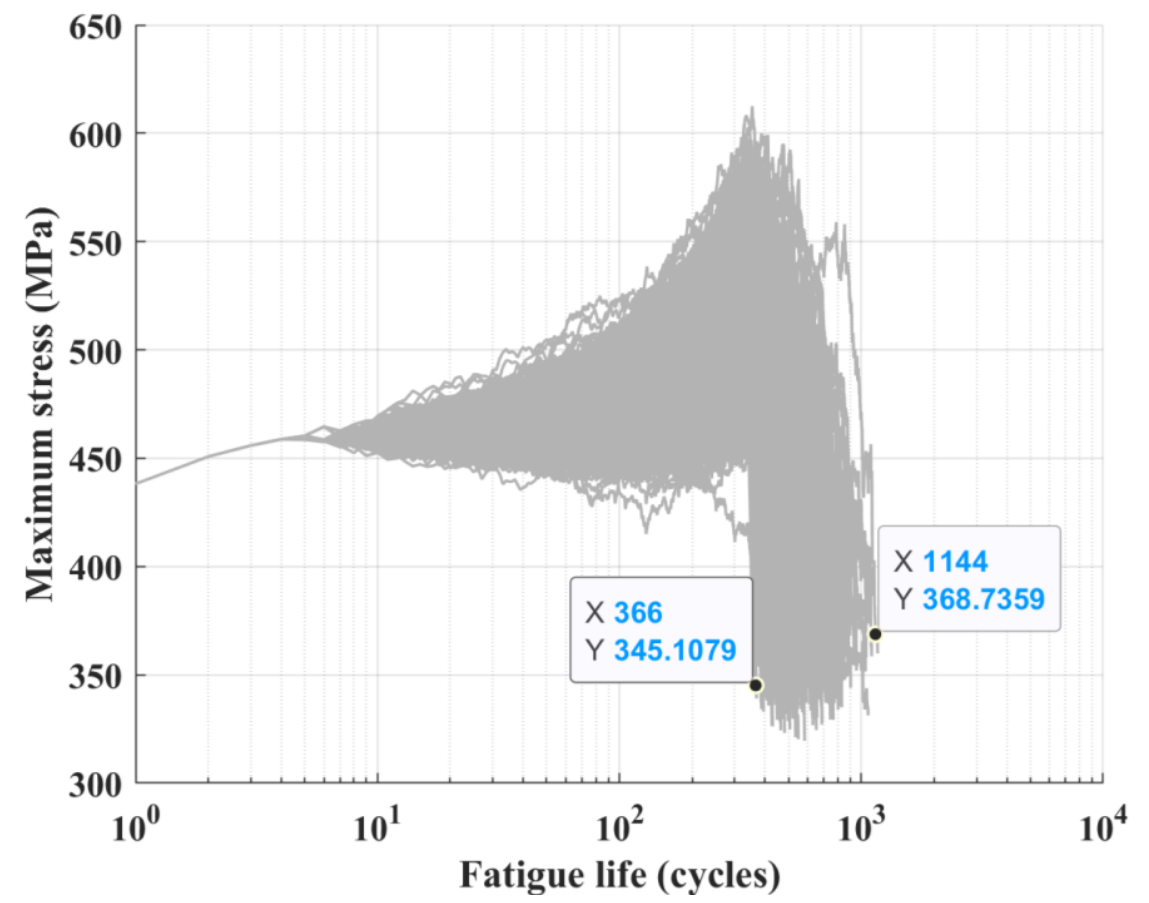

Figure 9. 5 MCMC estimated loading cycle versus the scatter-band of experimentally observed maximum stress amplitude of EN-F62 test case.

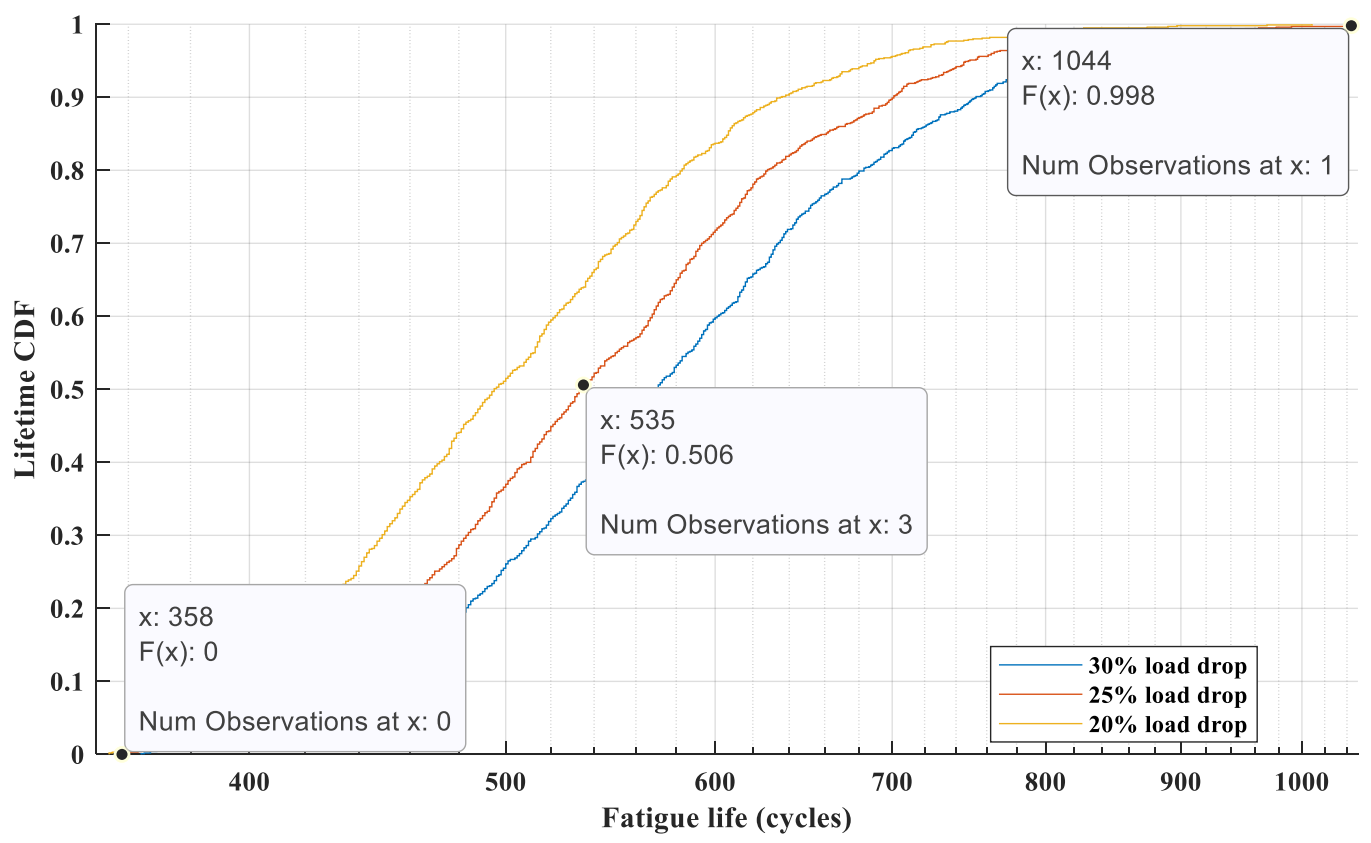

Figure 9. 6 Loading cycle versus estimated lifetime CDF, estimated based on the scatter-band shown in Figure 9.5 and using different load-drop failure criterion. 


\section{Summary}

Following are the major conclusions and takeaways from the FY-20 environmental fatigue research work conducted at ANL.

\subsection{Major Conclusions}

a) 82/182 DMW shows significant cyclic hardening/softening behavior under cyclic loading. This leads to a significant cyclic variation of material properties such as elastic modulus, offset-strain yield stress and kinematic hardening parameters.

b) A combined use of AI/ML technology (such as of TensorFlow AI/ML library) along with computational/solid mechanics-based approach can help to automatically estimate cyclic stressstrain curves and associated material parameters for hundreds of fatigue cycles.

c) Cyclic-plasticity based constitutive relation and associated analytical model can predict the cyclic stress not only under constant amplitude loading but also under variable amplitude loading with load-sequence effect. The results show that conventional tensile-test-based fixed material parameters wrongly predict the stress (both amplitude and hysteresis behavior) under cyclic loading. This necessitate the use of cyclic plasticity-based models for more accurate life estimation of reactor components.

d) AI/ML/DL technique (such as Scikit-learn and/or Keras libraries) can accurately predict unmeasurable time-series (cyclic) sensor signals from other sensor measurements. Out of the different approach tested, the Keras library along with ReLU activation function and k-mean based clustering of the fatigue cycles produces best results both under unsymmetrical (e.g. design-basis type) and symmetrical fatigue loading.

e) AI/ML technique (such as Scikit-learn based k-mean clustering technique) can help optimizing the environmental-fatigue testing parameters for controlling the strain amplitude and rates in PWR-water-test system, for which direct control of strain (through strain measurements) is not possible.

f) A Markov-Chain-Monte-Carlo based probabilistic approach could be used for probabilistic fatigue state and life estimation of DMW. This is based on both model and fatigue test-based data. 


\subsection{Major Takeaways}

1) A cyclic plasticity material model database for $82 / 182$ dissimilar metal weld, which can be readily shared with US nuclear industry and regulatory agency on request.

2) A well validated analytical modeling methodology to perform cycle-by-cycle stress prediction under both constant amplitude fatigue loading and variable amplitude fatigue loading (with loadsequence effect).

3) An AI/ML/DL based methodology to predict unmeasurable sensor signal (such as predicting timeseries or cyclic strain in an inaccessible autoclave environment) from other sensor signal. This type of approach can be used for estimating strain in real reactor components from other sensor readings.

4) An AI/ML based approach to improve the US capability on environmental fatigue testing. This is by improving ANL's existing environmental fatigue testing capacity to conduct ASME required strain-controlled tests (by controlling strain amplitudes and its rate), while not measuring the strain (due to the difficulty of placing an extensometer in a narrow autoclave of a PWR-water-test system).

5) A simulation and experiment based probabilistic modeling methodology for time-series fatigue state and life estimation of reactor metal such as dissimilar metal weld. 


\section{References}

1. https://scikit-learn.org/stable/

2. https://www.tensorflow.org/

3. https://keras.io/

4. Han, J., Kamber, M. and Pei, J., 2011. Data mining concepts and techniques third edition. The Morgan Kaufmann Series in Data Management Systems

5. Rasmussen, C.E. and Williams, C., 2006. Gaussian processes for machine learning., (MIT Press: Cambridge, MA).

6. Mohanty, S., Park, J.P., Franken, D., Listwan, J., Majumdar, S. and Natesan, K., “A Deterministic and Probabilistic Framework for Forecasting of Time-Series Damage States and Associated End-of-Life of a Pressurizer Water Reactor Surge Line under Design-Basis and/or Grid-Load-Following Loading Conditions,” Report No. ANL/LWRS-18/02. Argonne National Laboratory, September 2018. (https://www.osti.gov/biblio/1557591)

7. Mohanty, S., Jagielo, B., Iverson, W., Bahn, C., Soppet, W. K., Majumdar, S. and Natesan, K., "Online Stress Corrosion Crack and Fatigue Usages Factor Monitoring and Prognostics in Light Water Reactor Component: Probabilistic Modeling, System Identification and Data Fusion Based Big Data Analytics Approach September 2014, ANL/LWRS-14/2 (http://www.osti.gov/scitech/biblio/1168230)

8. Park, J.P., Mohanty, S., Bahn, C.B., Majumdar, S. and Natesan, K., 2020. Weibull and Bootstrap-Based Data-Analytics Framework for Fatigue Life Prognosis of the Pressurized Water Nuclear Reactor Component Under Harsh Reactor Coolant Environment. Journal of Nondestructive Evaluation, Diagnostics and Prognostics of Engineering Systems, 3(1).

9. Chattopadhyay, A. and Mohanty, S., "Gaussian Process Damage Prognosis under Random and Flight Profile Loading”, Data Mining in Systems Health Management: Detection, Diagnostics and Prognostics, Editors Ashok Srivastava and Jiawei Han, Chapman and Hall/CRC Press, Taylor and Francis Group

10. Mohanty, S., Chattopadhyay, A., and Rajadas, J. N., "Bayesian Nonlinear Model for Dynamic Strain Mapping and Real-time Damage State Estimation under Biaxial Random Fatigue Loading", AIAA Journal, Vol 50 (4), pp. 769-777, 2012.

11. Mohanty, S., Chattopadhyay, A., Peralta, P., and Das, S., "Off-Line and On-Line Fatigue Crack Growth Prediction Using Multivariate Gaussian Process, 2009, Journal of Experimental Mechanics

12. Liu, Y., Mohanty, S., Chattopadhyay, A., "Condition Based Structural Health Monitoring and Prognosis of Composite Structures under Uniaxial and Biaxial Loading, 2010, Journal of Nondestructive Evaluation, Volume 29, Number 3, 181-188

13. Mohanty, S., Chattopadhyay, A., Wei, J and Peralta, P., "Unsupervised Time-Series Damage State Estimation of Complex Structure Using Ultrasound Broadband Based Active Sensing", 2010, Structural Durability \& Health Monitoring Journal, vol.130, no.1, pp.101-124

14. Mohanty, S., Chattopadhyay, A., and Peralta, P., "Adaptive Residual Useful Life Estimation of a Structural Hotspot", 2009 Journal of Intelligent Material Systems and Structures, (special issues on SHM), 2010, February, Vol-21, pp: 321-335. 
15. Mohanty, S., Chattopadhyay, A., Wei, J and Peralta, P., "Real time Damage State Estimation and Condition Based Residual Useful Life Estimation of a Metallic Specimen under Biaxial Loading", 2009, Structural Durability \& Health Monitoring Journal, vol.5, no.1, pp.33-55.

16. Mohanty, S.., Das, S., Chattopadhyay, A., and Peralta, P., "Gaussian Process Time Series Model for Life Prognosis of Metallic Structure", Journal of Intelligent Material Systems and Structures, 2008, Vol. 20, No. 8, 887-896.

17. Mohanty, S., Listwan, J., and Park, J.P., "A System-Level Framework for Fatigue Life Prediction of a PWR Pressurizer-Surge-Line Nozzle under Design-basis Loading Cycles," Report No. ANL/LWRS-19/01. Argonne National Laboratory, September 2019. (https://www.osti.gov/biblio/1571258 )

18. Chaboche J. L., (1986) Time-independent constitutive theories for cyclic plasticity, International Journal of Plasticity 2: 149-188.

19. https://pandas.pydata.org/

20. Chopra, O., and Stevens, G. L., (2018), Effect of LWR Water Environments on the Fatigue Life of Reactor Materials (NUREG/CR-6909, Revision 1) - Final Report (https://www.nrc.gov/docs/ML1631/ML16319A004.pdf) 
This page intentionally left blank 


\section{Argonne}

Nuclear Engineering Division

Argonne National Laboratory

9700 South Cass Avenue, BIdg. 208

Argonne, IL 60439

www.anl.gov

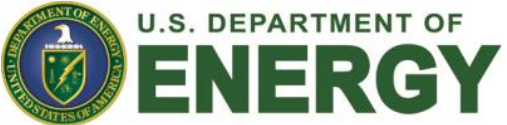

